Linköping Studies in Science and Technology. Licentiate Thesis No. 1897

\title{
Decentralized Estimation Using Conservative Information Extraction
}

Robin Forsling 



\section{Decentralized Estimation Using Conservative Information Extraction}

\section{Robin Forsling}

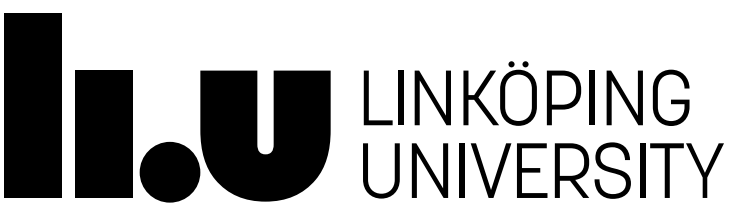


This is a Swedish Licentiate's Thesis.

Swedish postgraduate education leads to a Doctor's degree and/or a Licentiate's degree.

A Doctor's Degree comprises 240 ECTS credits (4 years of full-time studies).

A Licentiate's degree comprises 120 ECTS credits,

of which at least 60 ECTS credits constitute a Licentiate's thesis.

(c) EY-NC This work is licensed under a Creative Commons AttributionNonCommercial 4.0 International License.

https://creativecommons.org/licenses/by-nc/4.0/

Linköping studies in science and technology. Licentiate Thesis

No. 1897

Decentralized Estimation Using Conservative Information Extraction

Robin Forsling

robin.forslingliu.se

www. control.isy.liu.se

Department of Electrical Engineering

Linköping University

SE-581 83 Linköping

Sweden

ISBN 978-91-7929-724-4ＩSSN 0280-7971

Copyright @ 2020 Robin Forsling

Printed by LiU-Tryck, Linköping, Sweden 2020 
To Elin and Nils 



\section{Abstract}

Sensor networks consist of sensors (e.g., radar and cameras) and processing units (e.g., estimators), where in the former information extraction occurs and in the latter estimates are formed. In decentralized estimation information extracted by sensors has been pre-processed at an intermediate processing unit prior to arriving at an estimator. Pre-processing of information allows for the complexity of large systems and systems-of-systems to be significantly reduced, and also makes the sensor network robust and flexible. One of the main disadvantages of pre-processing information is that information becomes correlated. These correlations, if not handled carefully, potentially lead to underestimated uncertainties about the calculated estimates.

In conservative estimation the unknown correlations are handled by ensuring that the uncertainty about an estimate is not underestimated. If this is ensured the estimate is said to be conservative. Neglecting correlations means information is double counted which in worst case implies diverging estimates with fatal consequences. While ensuring conservative estimates is the main goal, it is desirable for a conservative estimator, as for any estimator, to provide an error covariance which is as small as possible. Application areas where conservative estimation is relevant are setups where multiple agents cooperate to accomplish a common objective, e.g., target tracking, surveillance and air policing.

The first part of this thesis deals with theoretical matters where the conservative linear unbiased estimation problem is formalized. This part proposes an extension of classical linear estimation theory to the conservative estimation problem. The conservative linear unbiased estimator (CLUE) is suggested as a robust and practical alternative for estimation problems where the correlations are unknown. Optimality criteria for the CLUE are provided and further investigated. It is shown that finding an optimal CLUE is more complicated than finding an optimal linear unbiased estimator in the classical version of the problem. To simplify the problem, a CLUE that is optimal under certain restrictions will also be investigated. The latter is named restricted best CLUE. An important result is a theorem that gives a closed form solution to a restricted best CLUE. Furthermore, several conservative estimation methods are described followed by an analysis of their properties. The methods are shown to be conservative and optimal under different assumptions about the underlying correlations.

The second part of the thesis focuses on practical aspects of the conservative approach to decentralized estimation in configurations where the communication channel is constrained. The diagonal covariance approximation is proposed as a data reduction technique that complies with the communication constraints and if handled correctly can be shown to preserve conservative estimates. Several information selection methods are derived that can reduce the amount of data being transmitted in the communication channel. Using the information selection methods it is possible to decide what information other actors of the sensor network find useful. 



\section{Populärvetenskaplig sammanfattning}

Estimeringsteori avser den del av statistik som behandlar skattare och skattningar av parametrar av olika slag, och har en historik som går flera sekel bakåt $\mathrm{i}$ tiden. Väl förankrat i matematiken har estimeringsteori blivit ett av fundamenten för teknik och teknikutveckling inom en rad områden. Exempel på tillämpningar är navigationssystem i flygplan och fartyg, radarers målföljningssystem, kommunikationssystem, bildbehandling och processreglering. Estimeringsteori är relevant, för att inte säga nödvändigt, inom i stort sett all tillämpning som har med utvinning av information (eng. information extraction) att göra.

Ett tillämpningsområde för estimeringsteori är sensornätverk som grovt sett är nätverk bestående av två sorters noder; sensorer (t.ex. radarer och kameror) och processorenheter (t.ex. skattare). Två distinkta klasser av sensornätverk är centraliserade sensornätverk och decentraliserade sensornätverk. I ett centraliserat sensornätverk kommuniceras all information som utvinns med hjälp av sensorer direkt till en central processorenhet där skattningen sköts. I den decentraliserade motsvarigheten skickas information som utvunnits av sensorer först till en lokal processorenhet som förbehandlar information till en skattning. Det är sedan den förbehandlade skattningen som kommuniceras till övriga processorenheter. Om det är praktiskt möjligt är en centraliserad lösning att föredra eftersom denna är optimal beträffande utvinning av information. Dock är denna lösning beroende av kritiska noder, och för större system väldigt svår att implementera i praktiken. Här krävs ofta någon form av förbehandling av information såsom sker i en decentraliserad lösning. Den stora utmaningen med den senare är att förbehandling av information skapar beroende mellan de skattningar som skickas runt i nätverket. Dessa beroenden, korrelationer, är generellt okända och måste tas om hand för att undvika att redan använd information återanvänds.

I den här avhandlingen studeras en speciell typ av skattningsproblem-det konservativa skattningsproblemet-vilket typiskt uppträder i just decentraliserade sensornätverk. Kortfattat innebär ett konservativt angreppssätt att osäkerheten hos en skattning aldrig får underskattas. Med andra ord är en konservativ ansats en slags säkra-före-det-osäkra-strategi. I fallet att korrelationer mellan skattningar är okända behöver en konservativ skattare kompensera för alla korrelationer som är möjliga för det specifika problemet. Samtidigt som det krävs att den sanna osäkerheten inte underskattas är det önskvärt att överskatta den sanna osäkerheten så lite som möjligt.

Konservativ skattning är relevant inom områden där flertalet kooperativa och kommunicerande plattformar tillsammans skattar samma objekt, exempelvis inom målföljning och luftrumsövervakning. I fallet luftrumsövervakning kan det röra sig om samarbetande plattformar, som observerar och skattar ett mål, och sedan sänder ut förbehandlade skattningar till övriga plattformar. I och med att skattningar innehåller både tidigare och nyligen utvunnen information skulle en eventuell ytterligare plattform som kopplar upp sig mot kommunikationskanalen snabbt få tillgång till den information som utvunnits.

Den första delen av avhandlingen formaliserar det konservativa skattningsproblemet med utgångspunkt i den klassiska motsvarigheten. Olika egenskaper 
av problemet tas upp, däribland optimalitetsaspekter. Det visar sig vara betydligt svårare att hitta optimala konservativa skattare jämfört med det klassiska fallet. En något förenklad optimal konservativ skattare definieras vilken rent strukturmässigt ligger närmare den klassiska motsvarigheten. Ett viktig resultat är formuleringen av ett matematiskt teorem som talar om hur en förenklad optimal konservativ skattare kan hittas. Vidare presenteras en rad konservativa skattningsmetoder och deras egenskaper utreds.

Fokus i den andra delen av avhandlingen är mer praktiska tillämpningar av det konservativa skattningsproblemet samt hur det slår på utvinningen av information i ett decentraliserat sensornätverk. Ett viktigt delmål är att minimera kommunikationskostnaden samtidigt som konservativa skattningar måste garanteras. Ett sätt är att endast utbyta en fördefinierad delmängd av osäkerhetsdatan för att på så vis avlasta kommunikationskanalen. Ett annat sätt är att använda sig av metoder vilka beräknar den information som övriga noder saknar. Ovan nämnda angreppssätt utreds via simuleringsstudier och visar sig vara effektiva både beträffande utnyttjandet av kommunikationskanalen samt ur perspektivet utvinning av information. 


\section{Acknowledgments}

At first I want to thank my supervisor Prof. Fredrik Gustafsson, and my cosupervisors Assoc. Prof. Gustaf Hendeby and Assoc. Prof. Zoran Sjanic. I am lucky to have your guidance, in particular when it comes to the balance between letting me work freely and providing me with directions to take. Thanks for all of your help, for your great ideas and for always having time for questions!

Thank you Assoc. Prof. Martin Enqvist, Head of Division, for allowing me to start at the Automatic Control Division. Your friendly treatment of people is one of the cornerstones for the pleasant mentality being present at Automatic Control, and thanks to all you other at Automatic Control for keeping up the good work environment. Ninna Stensgård, thanks for helping out with all the practical!

Proofreading of this thesis were performed by M.Sc. Alexander Rajula, M.Sc. Daniel Arnström, M.Sc. Daniel Bossér, B.Sc. Elin Lager, Lic. Erik Hedberg, M.Sc. Magnus Malmström and Lic. Per Boström-Rost. Your comments and suggestions have been invaluable for me! Extra thanks to Gustaf Hendeby for your universal support and help to tackle almost any problem. Whether it comes to technical issues, planning, estimation expertis, proofreading, scientific reasoning, beer tips, $\mathrm{AT}_{\mathrm{E} X} \mathrm{X}$ issues, and proofreading again-you are always there. Thanks for all your effort!

This work was supported by the Competence Center LINK-SIC, funded by Vinnova, which is a collaboration between the academia and the system-building industry of Sweden. I would like to thank the Center Director, Prof. Svante Gunnarsson, for letting me join LINK-SIC, and Sara Strömberg for arranging all the nice LINK-SIC workshops.

Thankfulness goes to my employer Saab AB for giving me the opportunity to even initiate this work. I especially want to thank Lars Pääjärvi, Gunnar Holmberg and Karolina Bergström at Saab AB.

Finally I would like to thank my family for always supporting me on my different journeys during life. Special gratefulness goes to my girlfriend Elin, our son Nils and our dog Ebba for being a part of my life, and especially Elin for always taking care of Nils and Ebba, and also for encouraging me. I love you!

Linköping, December 2020 Robin Forsling 



\section{Contents}

$\begin{array}{ll}\text { Notation } & \text { Xv }\end{array}$

1 Introduction 1

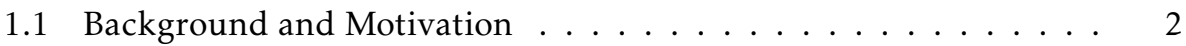

1.2 Related Work ... . . . . . . . . . . . . . . . 5

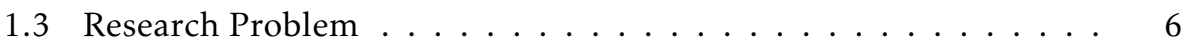

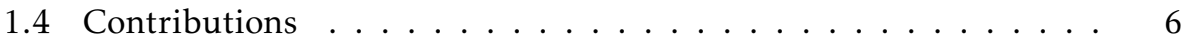

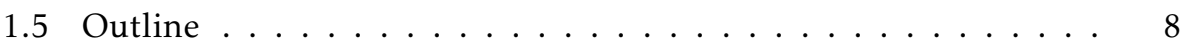

2 Linear Unbiased Estimation 9

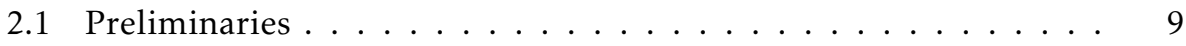

2.1 .1 Linear Models . . . . . . . . . . . . . . 10

2.1.2 Covariances Matrices and Ellipsoids . . . . . . . . . . 10

2.2 Best Linear Unbiased Estimator . . . . . . . . . . . . . . . . . . . . . 13

2.3 Linear Least Squares Estimation . . . . . . . . . . . . . . . . . . . . . . . . . 14

2.3.1 Properties . . . . . . . . . . . . . . 15

2.3.2 Information Form . . . . . . . . . . . . . . . 17

2.4 Linear Unbiased Fusion . . . . . . . . . . . . . . . . . . . . . . 18

2.4 .1 Correlated Estimates . . . . . . . . . . . . . 18

2.4.2 Fusion Under Known Cross-Correlations . . . . . . . . . 20

2.4.3 Fusion Under Zero Cross-Correlations . . . . . . . . . . 22

3 Conservative Linear Unbiased Estimation 23

3.1 Problem Formulation . . . . . . . . . . . . . . . . 24

3.1.1 The Linear Unbiased Estimation Problem Revisited . . . . 24

3.1.2 The Conservative Linear Unbiased Estimation Problem . . 25

3.1.3 Simplified Problem . . . . . . . . . . . . . . . 27

3.1 .4 Problem Properties ................ 28

3.2 Estimation Under Unknown Cross-Correlations . . . . . . . . . . . 28

3.3 Conservative Linear Unbiased Estimator . . . . . . . . . . . . . . 30

3.4 Best Conservative Linear Unbiased Estimator . . . . . . . . . . . . 32

3.4 .1 Properties and Insights . . . . . . . . . . . . . . 37

3.4.2 Relaxed Conservative Bounds . . . . . . . . . . 38 
3.5 Conservative Linear Least Squares Estimation . . . . . . . . . . . . 41

4 Conservative Linear Unbiased Fusion $\quad 45$

4.1 Fusion Under Completely Unknown Cross-Correlations . . . . . . 48

4.1.1 Completely Unknown Cross-Correlation . . . . . . . . . . 48

4.1 .2 Covariance Intersection . . . . . . . . . . . . . . 48

4.2 Fusion Under Correlated Information . . . . . . . . . . . . . . 54

4.2 .1 Correlated Information . . . . . . . . . . . . . . . 54

4.2.2 Inverse Covariance Intersection . . . . . . . . . . . . 55

4.3 Fusion Under Component-Wise Aligned Correlations . . . . . . . . 65

4.3.1 Component-Wise Aligned Correlations . . . . . . . . . . 65

4.3.2 Largest Ellipsoid Method . . . . . . . . . . . . 65

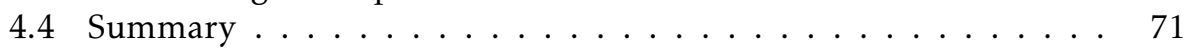

5 Diagonal Covariance Approximation $\quad \mathbf{7 5}$

5.1 Problem Formulation . . . . . . . . . . . . . . 76

5.1.1 Consequence of the Diagonal Covariance Approximation . 76

5.2 Methods for Preservation of Conservative Estimates . . . . . . . . 77

5.2.1 Post-Transmission Adaption of Covariance Intersection . . 77

5.2.2 Post-Transmission Scaling . . . . . . . . . . . . 78

5.2.3 Pre-Transmission Eigenvalue-Based Scaling . . . . . . . . 79

5.2.4 Pre-Transmission Optimization-Based Scaling . . . . . . . 80

5.2.5 Pre-Transmission Diagonal-Dominance Scaling . . . . . . . 81

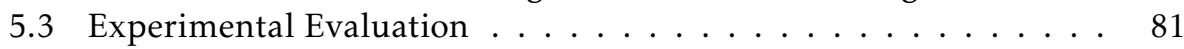

5.3.1 Simulation Scenario . . . . . . . . . . . . . . . . . 82

5.3.2 Evaluation Metrics ............... . . 82

5.3.3 Results and Discussion .............. 83

5.3 .4 Bandwidth Reduction . . . . . . . . . . . . . . 84

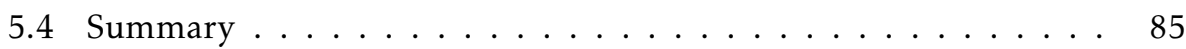

6 Selective Information Extraction $\quad 87$

6.1 Problem Formulation . . . . . . . . . . . . . . . 87

6.1.1 Considered Estimation Problem . . . . . . . . . . . 88

6.1.2 Motivating Example ................ 88

6.1.3 Fusion of Arbitrary Projections . . . . . . . . . . . 88

6.2 Information Selection Methods . . . . . . . . . . . . . . 89

6.2 .1 Aging Information . . . . . . . . . . . . . . 90

6.2.2 Largest Eigenvalue Method . . . . . . . . . . . . . . . 91

6.2.3 Transmitted Information Method . . . . . . . . . . . . . . . 91

6.2.4 Received Information Method . . . . . . . . . . . . . . 92

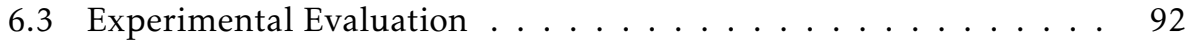

6.3.1 Considered Information Projections . . . . . . . . . . 92

6.3.2 Scenarios and Experimental Setup . . . . . . . . . . . 93

6.3 .3 Baseline Angle Analysis . . . . . . . . . . . . . . . 94

6.3 .4 Evaluation Metrics . . . . . . . . . . . . . . . 96

6.3 .5 Simulation Specifications . . . . . . . . . . . . 96 
6.3.6 Results and Discussion . . . . . . . . . . . . . 97

6.3.7 Bandwidth Reduction . . . . . . . . . . . . . . . . . . . . 99

6.4 Summary . . . . . . . . . . . . . . . . . 100

7 Concluding Remarks $\quad 101$

7.1 Summary and Conclusions . . . . . . . . . . . . . . 101

7.2 Future Work . . . . . . . . . . . . . . . . 102

$\begin{array}{ll}\text { Bibliography } & 105\end{array}$ 



\section{Abbreviations}

\begin{tabular}{cl}
\hline Abbreviation & Meaning \\
\hline l.h.s. & Left hand side \\
r.h.s. & Right hand side \\
W.r.t. & With respect to \\
ANEES & Average normalized estimation error squared \\
BLUE & Best linear unbiased estimator \\
CI & Covariance intersection \\
CKF & Centralized Kalman filter \\
CLUE & Conservative linear unbiased estimator \\
DCA & Diagonal covariance approximation \\
EKF & Extended Kalman filter \\
ICI & Inverse covariance intersection \\
ICIP & Inverse covariance intersection partial estimate form \\
ISM & Information selection methods \\
KF & Kalman filter \\
LE & Largest ellipsoid \\
LKF & Local Kalman filter \\
LS & Least squares \\
MCB & Minimum conservative bound \\
MSE & Mean squared error \\
NKF & Naïve Kalman filter \\
PD & Positive definite \\
PSD & Positive semi-definite \\
RMSE & Root mean squared error \\
WLS & Weighted least squares \\
\hline &
\end{tabular}




\section{General Mathematical Style}

\begin{tabular}{cl}
\hline Notation & Meaning \\
\hline$a$ & Scalar variable or parameter \\
$\mathbf{a}$ & Vector variable or parameter \\
$\mathbf{A}$ & Matrix variable or parameter \\
$\mathcal{A}$ & Space or set \\
$\mathcal{A}$ & Set of matrices \\
\hline
\end{tabular}

\section{Vector and Matrix Notation}

\begin{tabular}{cl}
\hline Notation & Meaning \\
\hline $\mathbf{0} \perp \mathbf{b}$ & Vector or matrix of zeros \\
$\langle\mathbf{a}, \mathbf{b}\rangle$ & $\mathbf{a}$ and $\mathbf{b}$ are orthogonal \\
$\|\mathbf{a}\|$ & Inner product of $\mathbf{a}$ and $\mathbf{b}$ \\
$\mathbf{A}^{-1}$ & Vector norm of $\mathbf{a}$ \\
$\mathbf{A}^{\top}$ & Inverse of $\mathbf{A}$ \\
$\mathbf{A}>0$ & Transpose of $\mathbf{A}$ \\
$\mathbf{A} \geq 0$ & $\mathbf{A}$ is positive definite \\
{$[\mathbf{A}]_{i j}$} & Element located at row $i$ and column $j$ in $\mathbf{A}$ \\
$\mathbf{I}$ & Identity matrix \\
$\operatorname{col}\left(\mathbf{a}_{1}, \ldots, \mathbf{a}_{n}\right)$ & Column stacking of $\mathbf{a}_{1}, \ldots, \mathbf{a}_{n}$ \\
$\operatorname{cov}(\mathbf{a})$ & True error covariance of $\mathbf{a}$ \\
$\operatorname{diag}\left(\mathbf{A}_{1}, \ldots, \mathbf{A}_{n}\right)$ & Diagonal matrix with $\mathbf{A}_{1}, \ldots, \mathbf{A}_{n}$ on the diagonal \\
$\operatorname{dim}(\mathbf{a})$ & Dimensionality of $\mathbf{a}$ \\
$\operatorname{det}(\mathbf{A})$ & Determinant of $\mathbf{A}$ \\
$\mathbf{E} \mathbf{A}$ & Expected value of $\mathbf{A}$ \\
$J(\cdot)$ & Matrix loss function \\
$\operatorname{rank}(\mathbf{A})$ & Rank of $\mathbf{A}$ \\
$\operatorname{tr}(\mathbf{A})$ & Trace of $\mathbf{A}$
\end{tabular}

\section{Set Notation}

\begin{tabular}{cl}
\hline Notation & Meaning \\
\hline $\mathbf{A} \in \mathcal{A}$ & $\mathbf{A}$ is an element of $\mathcal{A}$ \\
$\{\mathbf{A}, \mathbf{B}\}$ & Set with elements $\mathbf{A}$ and $\mathbf{B}$ \\
$\mathcal{A} \subseteq \mathcal{B}$ & $\mathcal{A}$ is a subset of $\mathcal{B}$ \\
$\mathcal{A} \subset \mathcal{B}$ & $\mathcal{A}$ is a strict subset of $\mathcal{B}$ \\
$\mathcal{A} \cap \mathcal{B}$ & Intersection of $\mathcal{A}$ and $\mathcal{B}$ \\
$\mathcal{A} \cup \mathcal{B}$ & Union of $\mathcal{A}$ and $\mathcal{B}$ \\
$\mathcal{E}(\mathbf{c}, \mathbf{S})$ & Ellipsoid with shape matrix $\mathbf{S}$ centered at c \\
$\mathcal{E}_{\circ}(\mathbf{c}, \mathbf{S})$ & Boundary of $\mathcal{E}(\mathbf{c}, \mathbf{S})$ \\
$\mathbb{R}$ & Set of real numbers \\
$\mathbb{R}^{n}$ & Set of real-valued $n$-dimensional vectors \\
$\mathbb{R}^{n \times m}$ & Set of real-valued $n \times m$ matrices \\
\hline
\end{tabular}




\section{Estimation AND Modeling}

\begin{tabular}{|c|c|}
\hline Notation & Meaning \\
\hline$k$ & Time index \\
\hline$k \mid l$ & Time indexing of filtered quantity \\
\hline$n_{\imath}$ & Dimensionality of $\mathbf{H}_{\imath} \mathbf{x}$ \\
\hline$n_{x}$ & Dimensionality of $\mathbf{x}$ \\
\hline$n_{y}$ & Dimensionality of $\mathbf{y}$ \\
\hline $\mathbf{e}_{l}$ & Additive noise of $\hat{\mathbf{z}}_{l}$ \\
\hline $\mathbf{v}$ & Additive noise of $\mathbf{y}$ \\
\hline $\mathbf{w}$ & Process noise \\
\hline $\mathbf{x}$ & True state \\
\hline$\hat{\mathbf{x}}$ & Full estimate of $\mathbf{x}$ \\
\hline$\tilde{\mathbf{x}}$ & True error $\hat{\mathbf{x}}-\mathbf{x}$ \\
\hline $\mathbf{y}$ & Data or measurement \\
\hline$\hat{\mathbf{z}}_{l}$ & Partial estimate $\imath$ of $\mathbf{x}$ \\
\hline$\hat{\mathbf{z}}_{J}$ & Joint state estimate $\operatorname{col}\left(\hat{\mathbf{z}}_{1}, \ldots, \hat{\mathbf{z}}_{N}\right)$ \\
\hline $\mathrm{C}_{1}$ & Error covariance of $\hat{\mathbf{z}}_{l}$ \\
\hline $\mathrm{C}_{J}$ & Error covariance of $\hat{\mathbf{z}}_{J}$ \\
\hline$\overline{\mathbf{C}}_{J}$ & Conservative bound of $\mathbf{C}_{J}$ \\
\hline$\overline{\mathbf{C}}_{J}^{*}$ & Minimum conservative bound of $\mathbf{C}_{J}$ \\
\hline $\mathbf{H}$ & Mapping from $\mathcal{X}$ to $\mathcal{Y}$ or mapping from $\mathcal{X}$ to $\mathcal{Z}$ \\
\hline $\mathbf{H}_{2}$ & Mapping from $\mathcal{X}$ to $\mathcal{Z}_{1}$ \\
\hline $\mathbf{H}_{J}$ & Mapping from $\mathcal{X}$ to space of $\mathbf{H}_{J} \mathbf{x}$ \\
\hline $\mathbf{K}$ & Estimation gain \\
\hline $\mathbf{K}_{\imath}$ & Fusion gain of $\hat{\mathbf{z}}_{l}$ \\
\hline $\mathbf{K}_{J}$ & Joint fusion gain \\
\hline $\mathbf{P}$ & Error covariance reported by the estimator $\hat{\mathbf{x}}$ \\
\hline$\overline{\mathbf{P}}$ & Conservative bound of $\operatorname{cov}(\hat{\mathbf{x}})$ \\
\hline$\overline{\mathbf{P}}^{*}$ & Minimum conservative bound of $\operatorname{cov}(\hat{\mathbf{x}})$ \\
\hline Q & Process noise covariance or correlation matrix \\
\hline $\mathbf{R}$ & Error covariance of $\mathbf{y}$ \\
\hline$\overline{\mathbf{R}}$ & Conservative bound of $\mathbf{R}$ \\
\hline$\overline{\mathbf{R}}^{*}$ & Minimum conservative bound of $\mathbf{R}$ \\
\hline $\mathcal{P}_{\mathbf{H}}$ & Projection matrix projecting onto $\mathcal{R}(\mathbf{H})$ \\
\hline $\mathcal{R}(\mathbf{H})$ & Space spanned by columns vectors of $\mathbf{H}$ \\
\hline $\mathcal{X}$ & State space of $\mathbf{x}$ \\
\hline $\mathcal{Y}$ & Space of $\mathbf{y}$ \\
\hline $\mathcal{Z}_{1}$ & State space of $\mathbf{H}_{l} \mathbf{x}$ \\
\hline $\mathbb{C}$ & Set to which $\mathbf{C}_{J}$ belong \\
\hline$\overline{\mathbb{C}}$ & Set to which all conservative bounds of $\mathbf{C}_{J}$ belong \\
\hline $\mathfrak{p}$ & Set to which $\operatorname{cov}(\hat{\mathbf{x}})$ belong \\
\hline$\overline{\mathfrak{p}}$ & Set to which all conservative bounds of $\operatorname{cov}(\hat{\mathbf{x}})$ belong \\
\hline $\mathfrak{k}$ & Set to which $\mathbf{R}$ belong \\
\hline$\overline{\mathfrak{R}}$ & Set to which all conservative bounds of $\mathbf{R}$ belong \\
\hline $\mathfrak{S}^{+}$ & Set of all symmetric positive semi-definite matrices \\
\hline $\mathfrak{S}^{++}$ & Set of all symmetric positive definite matrices \\
\hline
\end{tabular}





\section{1}

Introduction

TNFORMATION EXTRACTION is a fundamental aspect of almost every system deploying sensors. In many situations the information has been pre-processed and exchanged such that the actual new information extracted by the sensors becomes impossible to keep track of. Hence it follows that there is an imminent risk of reusing already used information, thus overestimating the information that has been extracted. Overestimating information is a logical fallacy and may have fatal consequences since it is equivalent to underestimating the uncertainty. As an example, think of a target being tracked where the same information about the target is being used multiple times to reduce the uncertainty about the target. At some point the calculated uncertainty about the target might become very small despite the fact that no new information has been extracted.

This work deals with an estimation problem where information is being used efficiently while it is avoided to reuse previously used information. Estimators which guarantee the usage of no more information than the actual extracted information are called conservative. The more conservative an estimator is, the more it underestimates the available information and hence the more it overestimates the uncertainty. The questions below are relevant in this scope:

- When is it possible to guarantee conservative estimates such that already used information is not being reused?

- Does the need for conservativeness depend on the situation?

- How can the information extraction be optimized while ensuring conservative estimators? 


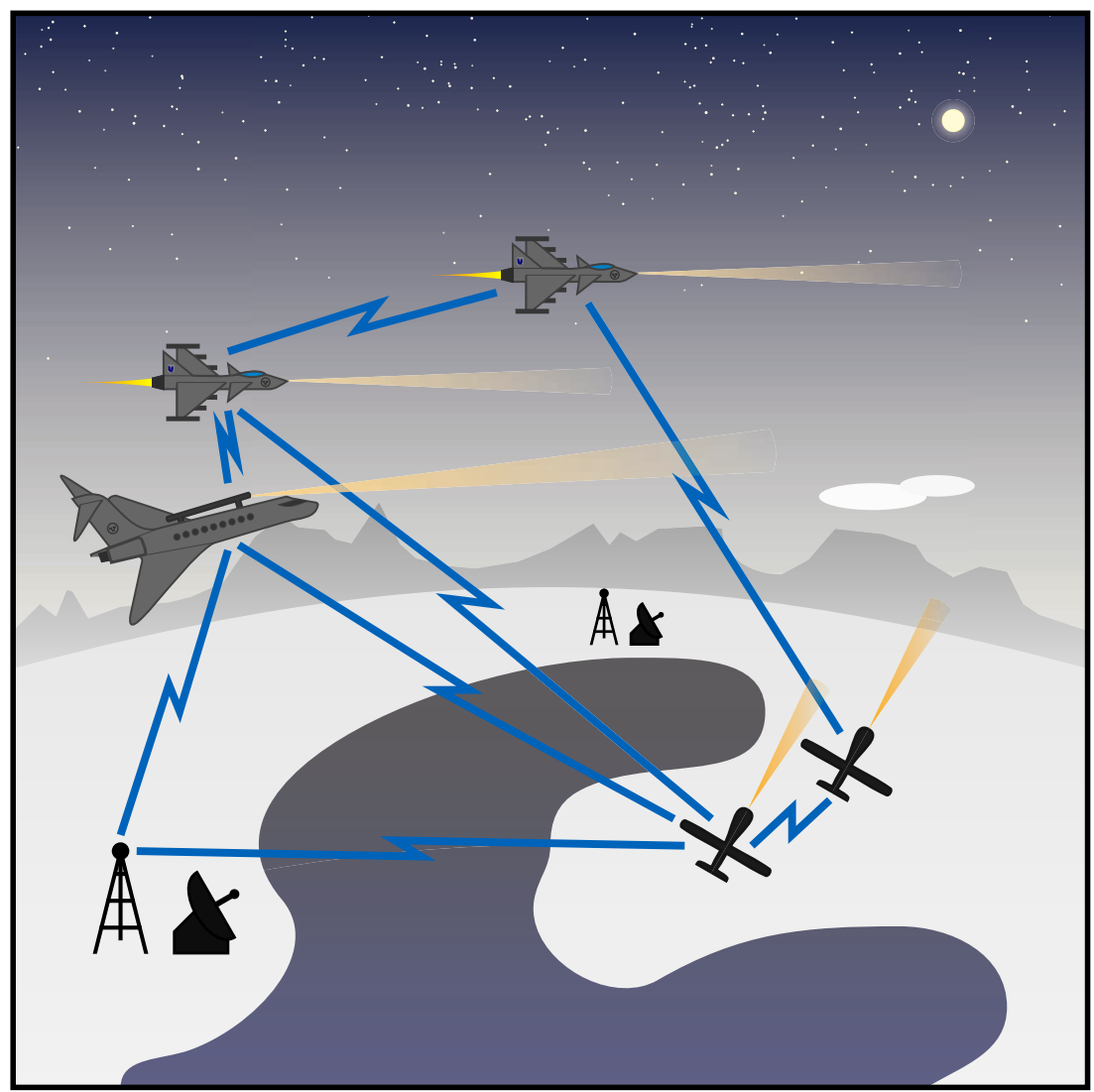

Figure 1.1: Motivating example. A number of heterogenous agents use their onboard sensors (yellow cones) to extract information and exchange estimates via a datalink (blue lines).

\subsection{Background and Motivation}

Consider the situation depicted in Figure 1.1. Multiple heterogenous agents are acquiring information about a target using onboard sensor. The measurements are pre-processed into estimates of the target state ${ }^{1}$ and the estimates are exchanged between the agents of the network [6]. Information is continuously passed around in the network. Hence, eventually there will be dependencies between the estimates received and the estimates transmitted-the estimates are cross-correlated. Cross-correlations, if not handled correctly, come with the sideeffect that previously used information is reused.

The example illustrates that when information is allowed to be communicated arbitrarily in a network, there must be some mechanism for handling the circulating information to prevent information from being double counted. If informa-

\footnotetext{
${ }^{1}$ E.g., target position and velocity.
} 


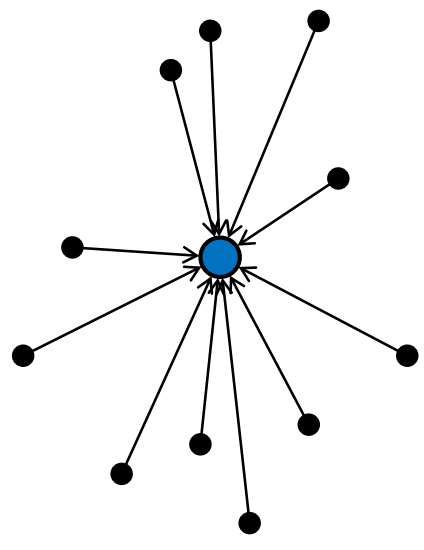

(a) Centralized sensor network.

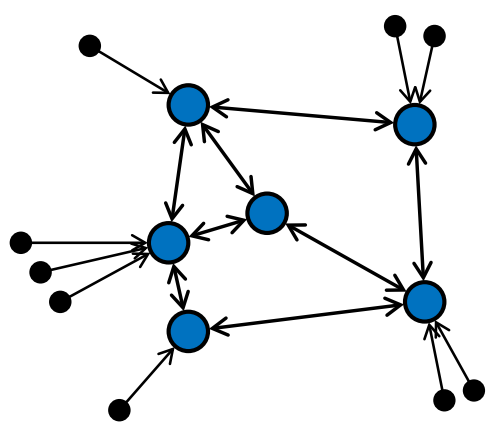

(b) Decentralized sensor network.

Figure 1.2: Comparison of different sensor network architectures. Black circles refer to sensor nodes and the blue larger circles refer to fusion nodes (processing units). The flow of information is indicated by the arrows.

tion is double counted the perceived uncertainty about the state being estimated is decreased despite no new information has actually been added, something that can potentially lead to diverging estimates [23].

Situations like the one in Figure 1.1 are common for military systems and systems-of-systems. Each agent or system is built up by various subsystems having their own information processing units and the different systems communicate information with other agents and systems.

\section{Network Architecture Aspects}

Now, one might ask: Is it not possible to design the communication network such that only uncorrelated measurements are exchanged? The answer to this is yes, at least in theory. Then one ends up with a centralized network where the unprocessed and uncorrelated measurements are fed directly to the processing unit, i.e., the data fusion node. A centralized network architecture is in sharp contrast to a decentralized network architecture, as the one exemplified in Figure 1.1. Both cases are illustrated schematically in Figure 1.2.

The centralized architecture allows for all information extracted by the sensors to be utilized, thus making it optimal. On the downside, the centralized architecture is vulnerable to failure of critical nodes, and for large systems and networks the combinatorial complexity will soon explode if information is not allowed to be pre-processed at intermediate steps [37].

According to [17] a decentralized sensor network is characterized by the following three constraints: 
1. No single central node.

2. Communication of data is only allowed on a node-to-node basis.

3. Nodes only have access to the local topology, i.e., nodes only know their nearest neighbors.

The decentralized architecture embeds measurements within estimates which makes information extraction more difficult. Meanwhile, the decentralized architecture offers robustness, since there will be no critical nodes, and a high level of modularity as nodes connected to the network only need to consider estimates given in a common reference frame [23].

Distributed architectures are typically distinguished from both centralized and decentralized architectures [8], and fall somewhere in between these two. However, in this scope the distributed sensor network is simply regarded as a special case of the decentralized sensor network since the methods used in decentralized estimation problems can also be used in distributed estimation problems. By fully decentralized sensor networks we mean decentralized sensor networks where the fusion nodes have no other knowledge about the communicated estimates than that they can be cross-correlated.

Today, defense systems involve integration of systems and systems-of-systems which imply an extremely high level of complexity. One way to reduce complexity is to deploy a decentralized sensor network where local information is first pre-processed into estimates, and where the estimates are then exchanged between the agents of the network. For large scale systems a decentralized architecture at some level is required [43]. In case of military applications decentralized sensor networks are further motivated from a robustness perspective [23].

\section{Estimation Aspects}

Denote by $\hat{\mathbf{x}}$ an estimate of the true state $\mathbf{x}$. Cross-correlated estimates, e.g., as implied by a decentralized network, are in this work handled by conservative estimators that approximate the true error $\tilde{\mathbf{x}}=\hat{\mathbf{x}}-\mathbf{x}$ by providing an upper bound $\mathbf{P}$ on the true error covariance $\operatorname{cov}(\hat{\mathbf{x}})=\mathrm{E} \tilde{\mathbf{x}} \tilde{\mathbf{x}}^{\top}$, see Figure 1.3. The main drawback of conservative estimates is that information will be lost, due to the fact that information is inversely proportional to the error covariance. The gap between $\mathbf{P}$ and $\operatorname{cov}(\hat{\mathbf{x}})$ is directly related to the loss of information that follows from the conservative bound.

The discussion above leads us to the following objectives in conservative estimation theory:

- Ensure conservative estimates. Guarantee that the approximated error covariance is an upper bound on the true error covariance. This corresponds to ensuring that $\mathbf{P}$ encloses the complete $\operatorname{cov}(\hat{\mathbf{x}})$ in Figure 1.3.

- Optimize information extraction. Extract as much information as possible about the object of interest. This corresponds to minimizing the gap between $\mathbf{P}$ and $\operatorname{cov}(\hat{\mathbf{x}})$ in Figure 1.3. 


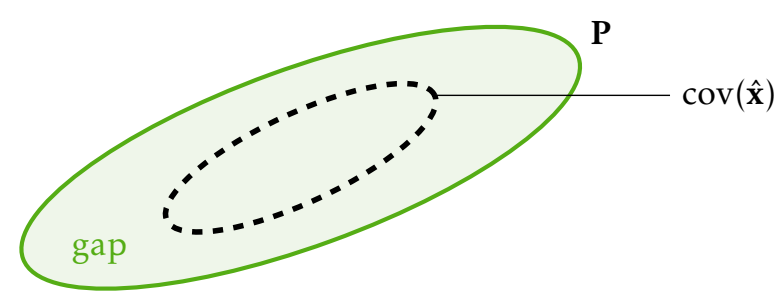

Figure 1.3: The ellipses of the true error covariance $\operatorname{cov}(\hat{\mathbf{x}})$ and a conservative bound $\mathbf{P}$. The gap between $\operatorname{cov}(\hat{\mathbf{x}})$ and $\mathbf{P}$ is related to the loss of information.

\section{Communication Aspects}

Both centralized and decentralized sensor networks require some sort of communication mechanism so that data can be exchanged throughout the network. The communication channel will be referred to as the datalink. In case of a centralized sensor network all data has to be transmitted from the sensors to one or several central node(s) which for large scale networks put high demands on the total bandwidth of the datalink [67].

The bandwidth utilization can be reduced by deploying a decentralized architecture where local sensor data is first aggregated into local estimates containing information extracted from both historical and current measurements. However, even in these setups care has to be taken regarding the availability of the datalink $[30,67]$. The datalink and the bandwidth allocation will therefore be of concern in this work.

\subsection{Related Work}

This thesis considers the usage of conservative estimation methods to handle estimation scenarios where unwanted cross-correlations arise, e.g., the decentralized estimation problem. Conservative estimation is not the only way to handle decentralized estimation problems in general. Methods based on information filtering approaches are suggested in $[11,42,44,46]$. In $[3,64]$ the authors propose algorithms that compensate for the cross-correlations under the assumption that bookkeeping of the cross-correlations is possible. A decorrelation procedure is proposed in [65] for the removal of previously exchanged information. Further methods for decorrelation of data have been suggested in $[40,41,51]$.

The approaches in $[28,55]$ are based on modeling of the cross-correlations. The authors of $[52,61]$ utilize certain samples to represent the cross-correlations. Decentralized estimation using square-root decompositions of covariance matrices is suggested in [53]. Furthermore, different distributed Kalman filtering schemes are derived in $[16,57]$, and in [50] an algorithm based on consensus convergence is developed.

Fully decentralized sensor networks in practice mean none of the above mentioned methods can be used. Therefore these networks demand some sort of 
conservative approach [23]. The main methods of conservative linear estimation are covariance intersection (CI, [22]), inverse covariance intersection (ICI, [49]), and the largest ellipsoid (LE, [5]) method. Conservative methods are compared in [2].

\subsection{Research Problem}

At a mathematical level, the conservative estimation problem arises due to the following: Two estimates $\hat{\mathbf{z}}_{1}$ and $\hat{\mathbf{z}}_{2}$ of the same true state $\mathbf{x}$ are provided, where their error covariances are given by $\operatorname{cov}\left(\hat{\mathbf{z}}_{1}\right)=\mathbf{C}_{1}$ and $\operatorname{cov}\left(\hat{\mathbf{z}}_{2}\right)=\mathbf{C}_{2}$, respectively. The cross-covariance, which describes how the two estimates vary relative to each other and hence tells us how much information is shared between the estimates, is given by $\operatorname{cov}\left(\hat{\mathbf{z}}_{1}, \hat{\mathbf{z}}_{2}\right)=\mathbf{C}_{12}$. In total the covariance structure of the two estimates is fully described by

$$
\operatorname{cov}\left(\left[\begin{array}{l}
\hat{\mathbf{z}}_{1} \\
\hat{\mathbf{z}}_{2}
\end{array}\right]\right)=\left[\begin{array}{cc}
\mathbf{C}_{1} & \mathbf{C}_{12} \\
\mathbf{C}_{21} & \mathbf{C}_{2}
\end{array}\right] .
$$

While the goal is to merge $\hat{\mathbf{z}}_{1}$ and $\hat{\mathbf{z}}_{2}$ to yield an improved estimate $\hat{\mathbf{x}}$, a common problem encountered in decentralized data fusion is that the cross-covariance $\mathbf{C}_{12}=\mathbf{C}_{21}^{\top}$ is unknown with the accompanying risk of double counting information when producing the estimate $\hat{\mathbf{x}}$.

The conservative criterion is defined as

$$
\mathbf{P} \geq \operatorname{cov}(\hat{\mathbf{x}}),
$$

where $\mathbf{P}$ here is the error covariance reported by the estimator, and $\mathbf{P} \geq \operatorname{cov}(\hat{\mathbf{x}})$ denotes the difference $\mathbf{P}-\operatorname{cov}(\hat{\mathbf{x}}) \geq \mathbf{0}$ is positive semi-definite. The main task in conservative estimation is to calculate $\hat{\mathbf{x}}$ with an error covariance $\mathbf{P} \geq \operatorname{cov}(\hat{\mathbf{x}})$. Also, the gap between $\mathbf{P}$ and $\operatorname{cov}(\hat{\mathbf{x}})$ should be kept at a minimum.

The research problem is illustrated Figure 1.4. The main task is to study the conservative estimation problem with a focus on information extraction aspects and conservativeness. The following subtasks will be addressed:

- Give insights into the conservative estimation problem, using examples, geometrical interpretations and applications.

- Provide an extension of classical linear estimation theory to the conservative linear estimation problem.

- Formalize optimality criteria for the conservative linear estimation problem.

- Summarize conservative estimation algorithms and their applications.

\subsection{Contributions}

Some of the contributions of this thesis are new theory and insights (Chapter 3 and Chapter 4), and some have already been published (Chapter 5 and Chapter 6). 
Input estimates

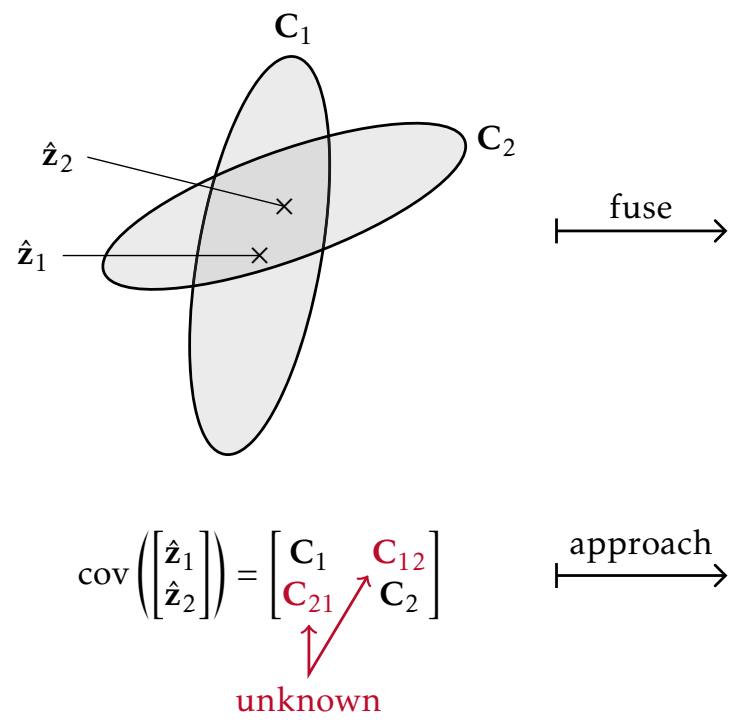

\section{Conservative estimation}

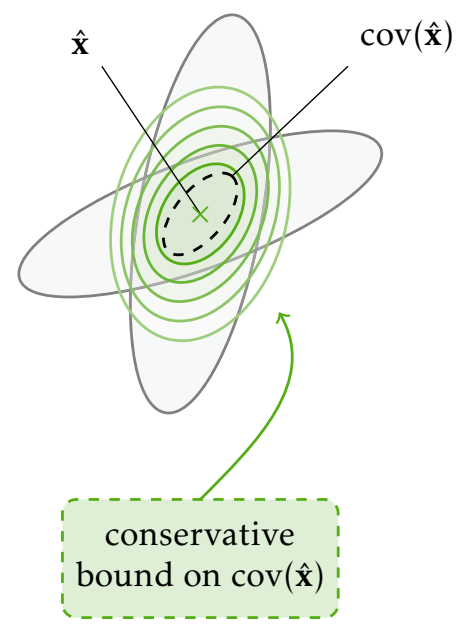

Figure 1.4: The conservative estimation problem. In conservative estimation the problem of not having access to $\mathbf{C}_{12}$ is handled by ensuring an upper bound on the true covariance $\operatorname{cov}(\hat{\mathbf{x}})$.

The main contribution of Chapter 3 is the introduction of the conservative linear unbiased estimator (CLUE). Other important contributions are two theorems. The first states the conditions under which a distinct optimal CLUE can be found. The second theorem is a conservative version of the Gauss-Markov theorem.

The main contributions of Chapter 4 are theorems stating under which circumstances CI, ICI and LE are optimal. Other important contributions are the partial estimate forms of ICI and LE.

The contents of Chapter 5 have been published in:

Robin Forsling, Zoran Sjanic, Fredrik Gustafsson, and Gustaf Hendeby. Consistent distributed track fusion under communication constraints. In Proceedings of the 22nd IEEE International Conference on Information Fusion, Ottawa, Canada, July 2019.

In this contribution a decentralized sensor network is considered in which communication constraints are present. The diagonal covariance approximation (DCA) is introduced as a way to reduce the bandwidth utilization of a datalink. Several methods are proposed that are able to ensure conservative estimates under the DCA.

Chapter 6 contains the publication:

Robin Forsling, Zoran Sjanic, Fredrik Gustafsson, and Gustaf Hendeby. Communication efficient decentralized track fusion using se- 
lective information extraction. In Proceedings of the 23rd IEEE International Conference on Information Fusion, Virtual Conference, July 2020.

This contribution also considers a decentralized sensor network under communication constraints. A number of information selection methods (ISM) are proposed which are able to selectively choose information that is useful within the network.

\subsection{Outline}

The thesis is organized as follows:

Chapter 1. Introduces the research problem and describes the contributions of the thesis.

Chapter 2. This chapter contains selected parts from linear estimation theory which constitute the theoretical basis for subsequent chapters.

Chapter 3. The conservative linear unbiased estimation problem is stated and from there the conservative linear unbiased estimator (CLUE) is defined. Optimality criteria are formalized. Conservative bounds and minimum conservative bounds are described. A conservative version of the Gauss-Markov theorem is given.

Chapter 4. In this chapter the focus lies on conservative linear unbiased fusion. Different conservative fusion methods are described and their properties are analyzed. Each method is related to a specific cross-correlation structure.

Chapter 5. Based on [12], this chapter introduces the diagonal covariance approximation together with several methods for preservation of conservativeness.

Chapter 6. Based on [13], this chapter provides methods for selectively choosing useful information. The proposed information selection methods can be used to reduce the bandwidth utilization of a datalink.

Chapter 7. In this chapter the thesis is concluded and future directions to take within the research field are suggested. 


\section{2}

\section{Linear Unbiased Estimation}

$\mathrm{T}$

HE ESTIMATION PROBLEM is approached using a Fisherian view. Hence the state of interest is a deterministic quantity $\mathbf{x}$, also regarded as the true state. The true state $\mathbf{x}$ is not known, but the data $\mathbf{y}$ which is related to $\mathbf{x}$ is available. Since $\mathbf{y}$ is corrupted by random noise it is handled as a random variable with covariance given by $\mathbf{R}=\operatorname{cov}(\mathbf{y})$. Only real-valued parameters and variables are considered.

An estimate of $\mathbf{x}$ is denoted by $\hat{\mathbf{x}}$ and $\mathbf{P}=\operatorname{cov}(\hat{\mathbf{x}})$ is the error covariance, or simply covariance, of the estimate. The estimate $\hat{\mathbf{x}}$ is a function of the random variable $\mathbf{y}$, hence $\hat{\mathbf{x}}$ is a random variable. Only unbiased estimators are considered, i.e., estimators for which $E \hat{\mathbf{x}}=\mathbf{x}$. The estimator itself is the mathematical rule that produces estimates. For a particular realization of $\mathbf{y}$ the estimator produces a particular numeric value of $\hat{\mathbf{x}}$. A somewhat vague notation is used, where $\hat{\mathbf{x}}$ is used both for the estimator and-its realization-the estimate.

The purpose of this chapter is to state the required background theory that will be used in subsequent chapters. The primary scope is linear estimation theory, restricted to only considering unbiased estimators. Some basic geometry and matrix theory is also introduced.

\subsection{Preliminaries}

The linear model is presented followed by a brief introduction to covariance matrices and their relationship to ellipsoids. Linear relationships are most often an idealization. Nevertheless, linear models are useful since they are in many cases approximately true and in general can give good insights to the properties of the problem [38]. 


\subsubsection{Linear Models}

A linear model relates the data $\mathbf{y}$ and the true state $\mathbf{x}$, where $\operatorname{dim}(\mathbf{y})=n_{y}$ and $\operatorname{dim}(\mathbf{x})=n_{x}$, according to

$$
\mathbf{y}=\mathbf{H x}+\mathbf{v},
$$

where $\mathbf{H}$ is an $n_{y} \times n_{x}$ matrix and $\mathbf{v}$ is additive random noise with covariance $\operatorname{cov}(\mathbf{v})=\mathbf{R}$. The relationship in (2.1) is often referred to as a (linear) measurement model. The noise is assumed zero-mean, i.e., $\mathrm{E} \mathbf{v}=\mathbf{0}$, such that

$$
\mathrm{E} \mathbf{y}=\mathrm{E}(\mathbf{H} \mathbf{x}+\mathbf{v})=\mathrm{E} \mathbf{H} \mathbf{x}+\mathrm{E} \mathbf{v}=\mathbf{H} \mathbf{x}
$$

where $E$ is the expected value operator. The zero-mean assumption is motivated in the case of, e.g., only using sensors for which any bias has been compensated. An estimate of $\mathbf{x}$ is given by $\hat{\mathbf{x}}$ which is also of dimensionality $n_{x}$.

If $\mathbf{x} \in \mathcal{X}$ and $\mathbf{y} \in \mathcal{Y}$ then $\mathbf{H}$ is the linear mapping

$$
\mathbf{H}: \mathcal{X} \longrightarrow \mathcal{Y}
$$

where $\mathcal{X}$ and $\mathcal{Y}$ denote vector spaces. In other words, $\mathbf{H}$ is the mapping from the state space $\mathcal{X}$ where the true state resides to the measurement space $\mathcal{Y}$.

\subsubsection{Covariances Matrices and Ellipsoids}

The notation $\operatorname{cov}(\mathbf{y})$ denotes the covariance of $\mathbf{y}$, hence

$$
\operatorname{cov}(\mathbf{y})=E(\mathbf{y}-E \mathbf{y})(\mathbf{y}-E \mathbf{y})^{\top} .
$$

Similarly, the notation $\operatorname{cov}\left(\mathbf{y}_{1}, \mathbf{y}_{2}\right)$ is used to define the cross-covariance between $\mathbf{y}_{1}$ and $\mathbf{y}_{2}$, i.e.,

$$
\operatorname{cov}\left(\mathbf{y}_{1}, \mathbf{y}_{2}\right)=E\left(\mathbf{y}_{1}-E \mathbf{y}_{1}\right)\left(\mathbf{y}_{2}-E \mathbf{y}_{2}\right)^{\top} \text {. }
$$

In particular

$$
\operatorname{cov}(\hat{\mathbf{x}})=\mathrm{E} \tilde{\mathbf{x}} \tilde{\mathbf{x}}^{\top},
$$

where $\tilde{\mathbf{x}}=\hat{\mathbf{x}}-\mathbf{x}$ is the true error of the estimate $\hat{\mathbf{x}}$.

With a few exceptions, the covariances matrices $\mathbf{R}$ dealt with herein are assumed to be positive definite (PD), i.e.,

$$
\mathbf{R}>\mathbf{0},
$$

where $>\mathbf{0}$ denotes the left hand side (1.h.s.) is PD. The set of all PD matrices is denoted $\mathfrak{S}^{++}$. The notion

$$
\mathbf{R}_{1} \geq \mathbf{R}_{2},
$$

is used to denote $\mathbf{R}_{1}-\mathbf{R}_{2} \geq \mathbf{0}$ where $\geq \mathbf{0}$ means the l.h.s. is positive semi-definite (PSD). A $n \times n$ PSD matrix $\mathbf{R} \in \mathfrak{S}^{+}$, where $\mathfrak{S}^{+}$is the set of all symmetric PSD matrices, can be factorized using an eigendecomposition defined as [26]

$$
\mathbf{R}=\mathbf{V} \Sigma \mathbf{V}^{\top}=\sum_{i=1}^{n} \lambda_{i} \mathbf{v}_{i} \mathbf{v}_{i}^{\top},
$$


where $\Sigma$ is a diagonal matrix containing the $i$ th eigenvalue $\lambda_{i} \geq 0$ on the $i$ th diagonal entry, and $\mathbf{V}$ is an orthogonal matrix having the corresponding eigenvector $\mathbf{v}_{i}$ as the $i$ th column.

The inverse of $\mathbf{R} \in \mathfrak{S}^{++}$can be written as

$$
\mathbf{R}^{-1}=\left(\mathbf{V} \Sigma \mathbf{V}^{\top}\right)^{-1}=\mathbf{V}^{-\top} \Sigma^{-1} \mathbf{V}^{-1}=\mathbf{V} \Sigma^{-1} \mathbf{V}^{\top}=\sum_{i=1}^{n} \lambda_{i}^{-1} \mathbf{v}_{i} \mathbf{v}_{i}^{\top},
$$

where the property $\mathbf{V}^{-1}=\mathbf{V}^{\top}$ of orthogonal matrices has been used. If $\mathbf{R}$ is PD and the multiplicity of each eigenvalue is one then the eigendecomposition is unique [19]. For PD matrices $\mathbf{R}_{1}$ and $\mathbf{R}_{2}$ it holds that [19]

$$
\mathbf{R}_{1} \geq \mathbf{R}_{2} \Longleftrightarrow \mathbf{R}_{2}^{-1} \geq \mathbf{R}_{1}^{-1}
$$

It must be noted that matrices are not always comparable. For example, given $\mathbf{A}_{1}$ and $\mathbf{A}_{2}$ of conformal size neither $\mathbf{A}_{1} \geq \mathbf{A}_{2}$ nor $\mathbf{A}_{2} \geq \mathbf{A}_{1}$ might hold. In this case we say that $\mathbf{A}_{1}$ and $\mathbf{A}_{2}$ are incomparable.

The rank of a matrix $\mathbf{A}$ is given by $\operatorname{rank}(\mathbf{A})$ and is equal to the number of linearly independent columns of $\mathbf{A}$ (or equivalently, the number of linearly independent rows of $\mathbf{A}$ ) [19]. An $n \times n$ matrix $\mathbf{A}$ is full rank if

$$
\operatorname{rank}(\mathbf{A})=n
$$

An $n \times m$ matrix $\mathbf{B}$ is said being full rank if

$$
\operatorname{rank}(\mathbf{B})=\min (n, m) .
$$

Transforming $\mathbf{y}$ using a linear operator $\mathbf{T}$ implies

$$
\begin{aligned}
E \mathbf{T y} & =\mathbf{T} E \mathbf{y}, \\
\operatorname{cov}(\mathbf{T y}) & =\mathbf{T} \operatorname{cov}(\mathbf{y}) \mathbf{T}^{\top} .
\end{aligned}
$$

\section{Ellipsoids}

Ellipsoids are described by a centering vector c and a symmetric shape matrix $\mathbf{S} \in \mathfrak{S}^{++}$. An ellipsoid $\mathcal{E}(\mathbf{c}, \mathbf{S})$ is given by the implicit form [7]

$$
\mathcal{E}(\mathbf{c}, \mathbf{S})=\left\{\mathrm{x} \in \mathbb{R}^{n} \mid(\mathrm{x}-\mathrm{c})^{\top} \mathbf{S}^{-1}(\mathrm{x}-\mathrm{c}) \leq 1\right\},
$$

which is a convex set of all points satisfying $(x-c)^{\top} S^{-1}(x-c) \leq 1$. The boundary of the same ellipsoid $\mathcal{E}_{\mathrm{o}}(\mathbf{c}, \mathbf{S})$ is defined as

$$
\mathcal{E}_{\mathrm{o}}(\mathrm{c}, \mathbf{S})=\left\{\mathrm{x} \in \mathbb{R}^{n} \mid(\mathrm{x}-\mathrm{c})^{\top} \mathbf{S}^{-1}(\mathrm{x}-\mathrm{c})=1\right\} .
$$

The eigendecomposition in (2.9) can be used to transform an arbitrary PD matrix $\mathbf{R}$ into a diagonal matrix using the similarity transformation $\mathbf{T}=\mathbf{V}^{-1}$, i.e.,

$$
\operatorname{TRT}^{\top}=\mathbf{V}^{-1} \mathbf{V} \Sigma \mathbf{V}^{\top} \mathbf{V}^{-\top}=\Sigma \text {. }
$$


An arbitrarily oriented covariance ellipsoid $\mathcal{E}\left(\mathbf{c}, \mathbf{V} \Sigma \mathbf{V}^{\top}\right)$ can hence also be described by

$$
\begin{aligned}
\mathcal{E}\left(\mathbf{c}, \mathbf{V} \Sigma \mathbf{V}^{\top}\right) & =\left\{\mathbf{x} \in \mathbb{R}^{n} \mid(\mathbf{x}-\mathbf{c})^{\top}\left(\mathbf{V} \Sigma \mathbf{V}^{\top}\right)^{-1}(\mathbf{x}-\mathbf{c}) \leq 1\right\} \\
& =\left\{\mathbf{x}^{\prime} \in \mathbb{R}^{n} \mid\left(\mathbf{x}^{\prime}-\mathbf{c}^{\prime}\right)^{\top} \Sigma^{-1}\left(\mathbf{x}^{\prime}-\mathbf{c}^{\prime}\right) \leq 1\right\} \\
& =\left\{\mathbf{x}^{\prime} \in \mathbb{R}^{n} \mid \sum_{i=1}^{n} \frac{\left\|\mathbf{x}^{\prime}-\mathbf{c}^{\prime}\right\|^{2}}{\lambda_{i}} \leq 1\right\},
\end{aligned}
$$

where $\mathbf{x}^{\prime}=\mathbf{V}^{\top} \mathbf{x}, \mathbf{c}^{\prime}=\mathbf{V}^{\top} \mathbf{c}$ and $\Sigma=\operatorname{diag}\left(\lambda_{1}, \ldots, \lambda_{n}\right)$. The ellipsoid in (2.18) is axis aligned with the coordinate frame used to represent $x^{\prime}$. If a covariance matrix $\mathbf{R}$ given in the $x$ coordinates is diagonal, then the covariance ellipsoid $\mathcal{E}(\mathbf{c}, \mathbf{R})$ is axis aligned with the coordinate frame used to represent $x$. Example 2.1 illustrates the relationship between covariance matrices, eigendecompositions and covariance ellipsoids.

The following relationships between algebra and geometry, given in [31], are important in this scope

$$
\begin{aligned}
& \mathbf{R}_{1} \geq \mathbf{R}_{2} \Longleftrightarrow \mathcal{E}\left(\mathbf{c}, \mathbf{R}_{1}\right) \supseteq \mathcal{E}\left(\mathbf{c}, \mathbf{R}_{2}\right), \\
& \mathbf{R}_{1}>\mathbf{R}_{2} \Longleftrightarrow \mathcal{E}\left(\mathbf{c}, \mathbf{R}_{1}\right) \supset \mathcal{E}\left(\mathbf{c}, \mathbf{R}_{2}\right),
\end{aligned}
$$

where $\mathrm{c}$ is the center of each ellipsoid.

\section{Example 2.1: Covariance Ellipses}

Assume the $2 \times 2$ covariance matrices

$$
\begin{aligned}
& \mathbf{R}_{1}=\left[\begin{array}{ll}
a & 0 \\
0 & d
\end{array}\right]=\lambda_{1} \mathbf{v}_{1} \mathbf{v}_{1}^{\top}+\lambda_{2} \mathbf{v}_{2} \mathbf{v}_{2}^{\top}, \\
& \mathbf{R}_{2}=\left[\begin{array}{ll}
a^{\prime} & b^{\prime} \\
b^{\prime} & d^{\prime}
\end{array}\right]=\beta_{1} \mathbf{u}_{1} \mathbf{u}_{1}^{\top}+\beta_{2} \mathbf{u}_{2} \mathbf{u}_{2}^{\top},
\end{aligned}
$$

where the pair $\lambda_{i}$ and $\mathbf{v}_{i}$, and the pair $\beta_{i}$ and $\mathbf{u}_{i}$, are the $i$ th eigenvalue and eigenvector of $\mathbf{R}_{1}$ and $\mathbf{R}_{2}$, respectively. Having $\mathbf{x}=\left[\begin{array}{ll}x_{1} & x_{2}\end{array}\right]^{\top}$, the matrix $\mathbf{R}_{2}$ can be transformed into a diagonal matrix given in the frame used to represent $x^{\prime}$ by letting

$$
x_{1}^{\prime}=\mathbf{u}_{1}^{\top} \mathrm{x}, \quad \mathrm{x}_{2}^{\prime}=\mathbf{u}_{2}^{\top} \mathrm{x},
$$

such that $x^{\prime}=\left[\begin{array}{ll}x_{1}^{\prime} & x_{2}^{\prime}\end{array}\right]^{\top}=\left[\begin{array}{ll}\mathbf{u}_{1}^{\top} x & \mathbf{u}_{2}^{\top} x\end{array}\right]^{\top}$.

The relationship between the covariance matrices $\mathbf{R}_{1}$ and $\mathbf{R}_{2}$ and their covariance ellipses $\mathcal{E}\left(\mathbf{c}_{1}, \mathbf{R}_{1}\right)$ and $\mathcal{E}\left(\mathbf{c}_{2}, \mathbf{R}_{2}\right)$, respectively, are illustrated in Figure 2.1. 


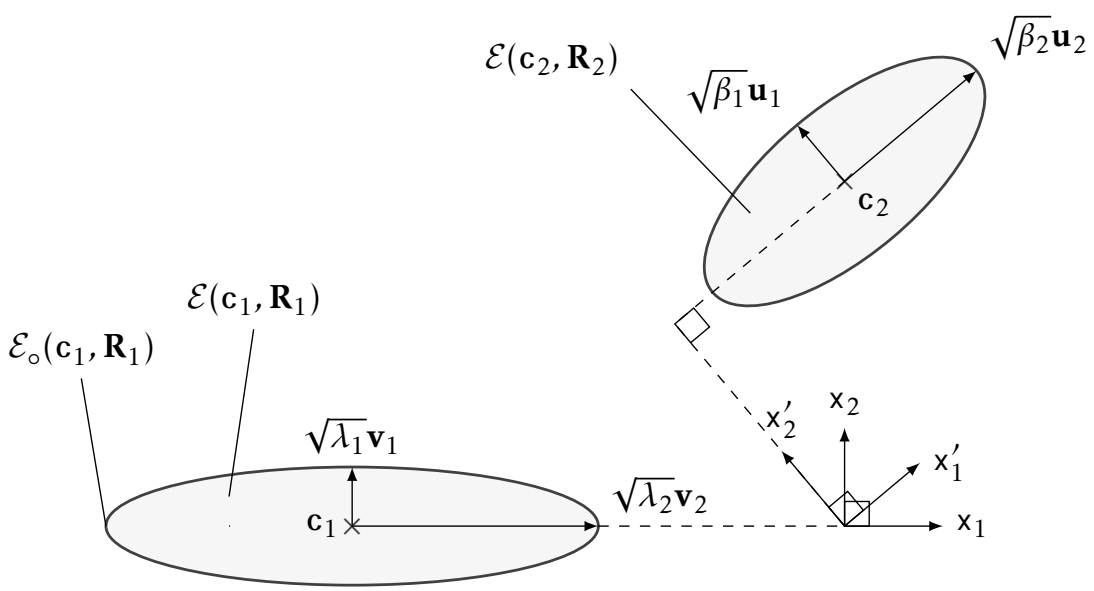

Figure 2.1: The covariances $\mathbf{R}_{1}$ and $\mathbf{R}_{2}$ represented as ellipses. The eigendecomposition of $\mathbf{R}_{1}$ and $\mathbf{R}_{2}$ are given by $\sum_{i=1}^{2} \lambda_{i} \mathbf{v}_{i} \mathbf{v}_{i}^{\top}$ and $\sum_{i=1}^{2} \beta_{i} \mathbf{u}_{i} \mathbf{u}_{i}^{\top}$, respectively. The ellipse boundary $\mathcal{E}_{\mathrm{o}}\left(\mathbf{c}_{1}, \mathbf{R}_{1}\right)$ is also illustrated.

\subsection{Best Linear Unbiased Estimator}

Producing just any arbitrary estimate $\hat{\mathbf{x}}$ from $\mathbf{y}$ is not necessarily useful by itself. Therefore the estimation problem needs to be narrowed down and more specifically defined. The first constraint adopted here is the linear condition

$$
\hat{\mathbf{x}}=\mathbf{K y} \text {, }
$$

where $\mathbf{K}$ is the estimation gain. Having $\hat{\mathbf{x}}$ linearly dependent on the data $\mathbf{y}$ has the nice implication that only knowledge about the first- and second-order statistical moments of $\mathbf{y}$ are required [26].

Secondly, the estimate should be unbiased, i.e.,

$$
E \hat{\mathbf{x}}=\mathbf{x}
$$

Finally, the estimate should be optimal with respect to (w.r.t.) some loss function. The most commonly adopted objective function is the mean squared error (MSE) defined as

$$
\mathrm{E}\|\hat{\mathbf{x}}-\mathbf{x}\|^{2}=\mathrm{E}\|\tilde{\mathbf{x}}\|^{2}=\mathrm{E} \tilde{\mathbf{x}}^{\top} \tilde{\mathbf{x}}=\operatorname{tr}\left(\mathrm{E} \tilde{\mathbf{x}} \tilde{\mathbf{x}}^{\top}\right),
$$

which is also the objective function used here.

The assumptions and conditions stated above bring us to the concept of the best linear unbiased estimator (BLUE) [29], which is the unbiased estimator that is linear in the data and produces estimates having the smallest MSE. In fact, as we will see soon, the BLUE has the stronger property that the smallest, in the PSD sense, covariance is achieved. The BLUE is defined in Definition 2.1 and is illustrated in Figure 2.2. 


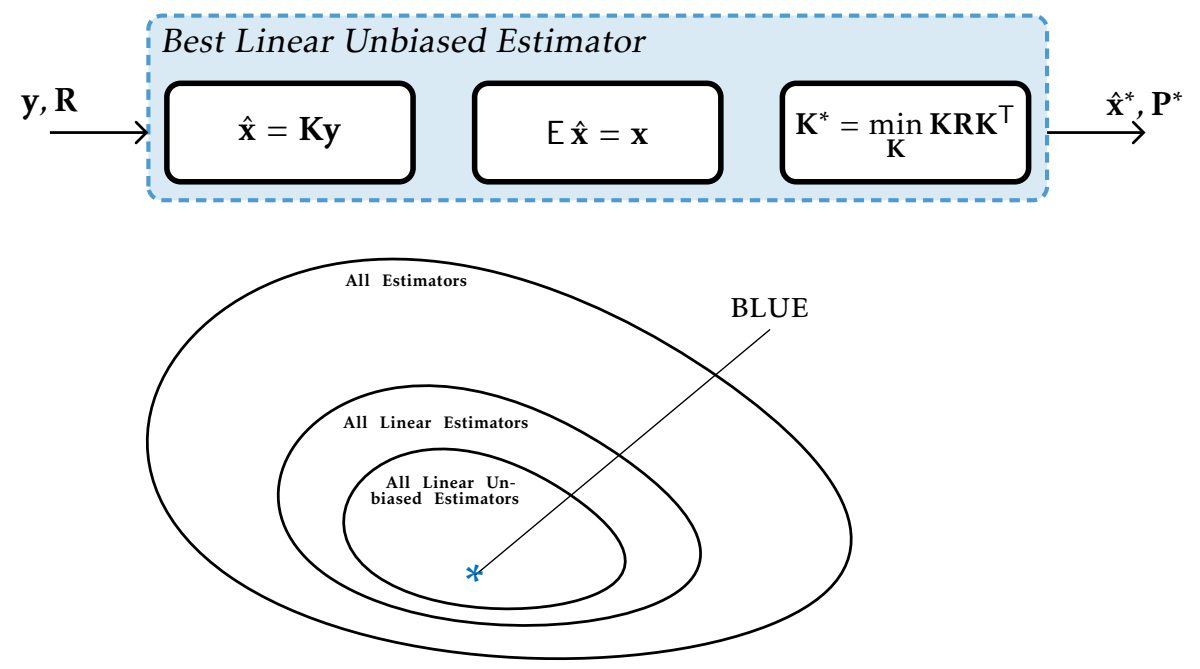

Figure 2.2: Illustration of the BLUE in relation to all other linear estimators.

Definition 2.1 (Best Linear Unbiased Estimator). Given is the noisy data y related to the true state $\mathbf{x}$. The unbiased estimator $\hat{\mathbf{x}}^{*}=\mathbf{K}^{*} \mathbf{y}$ is the best linear unbiased estimator (BLUE) if for all unbiased estimators $\hat{\mathbf{x}}=\mathbf{K y}$ it holds that

$$
\operatorname{cov}(\hat{\mathbf{x}}) \geq \operatorname{cov}\left(\hat{\mathbf{x}}^{*}\right) .
$$

\subsection{Linear Least Squares Estimation}

The least squares estimation method, originally introduced by Legendre [32] and Gauss [15] in the beginning of the 19th century, has found a vast amount of applications in estimation problems-and still do. The linear model of (2.1) is assumed. It is also assumed $\operatorname{rank}(\mathbf{H})=n_{x}$, where $n_{x} \leq n_{y}$.

In weighted least squares (WLS) estimation the aim is to find an estimate that minimizes the loss function

$$
J^{\mathrm{WLS}}(\mathbf{x})=\|\mathbf{y}-\mathbf{H} \mathbf{x}\|_{\mathbf{W}}^{2}=(\mathbf{y}-\mathbf{H} \mathbf{x})^{\top} \mathbf{W}(\mathbf{y}-\mathbf{H} \mathbf{x}),
$$

for a given weight matrix $\mathbf{W} \in \mathfrak{S}^{++}$and data $\mathbf{y}$. The weight matrix $\mathbf{W}$ allows for the components of $\mathbf{y}$ to be weighted non-uniformly when constructing the estimate $\hat{\mathbf{x}}$. The solution is given by [29]

$$
\hat{\mathbf{x}}=\underset{\mathbf{x}}{\arg \min }\|\mathbf{y}-\mathbf{H} \mathbf{x}\|_{\mathbf{W}}^{2}=\left(\mathbf{H}^{\top} \mathbf{W} \mathbf{H}\right)^{-1} \mathbf{H}^{\top} \mathbf{W} \mathbf{y},
$$

which is linear in the data, i.e., structurally equivalent to (2.20). The WLS estimate $\hat{\mathbf{x}}$ may be viewed as the vector that minimizes the length of the residual 
vector $\mathbf{W}^{\frac{1}{2}}(\mathbf{y}-\mathbf{H} \hat{\mathbf{x}})$ which is related to how well the estimate fits the data given the weights $\mathbf{W}$. If the data can be expressed according to (2.1), with $\mathbf{R}=\operatorname{cov}(\mathbf{y})$, the covariance of $\hat{\mathbf{x}}$ is given by [26]

$$
\operatorname{cov}(\hat{\mathbf{x}})=\left(\mathbf{H}^{\top} \mathbf{W H}\right)^{-1} \mathbf{H}^{\top} \mathbf{W} \mathbf{R W H}\left(\mathbf{H}^{\top} \mathbf{W H}\right)^{-1} .
$$

Denote by $\mathcal{R}(\mathbf{H})$ the space spanned by the columns of $\mathbf{H}$. A geometrical interpretation of the WLS solution is provided in Figure 2.3, where $\mathbf{y} \notin \mathcal{R}(\mathbf{H})$ and for simplicity $\mathbf{W}=\mathbf{I}$ is assumed. Using geometry it can be argued that the length of the estimation residual $\mathbf{y}-\mathbf{H} \hat{\mathbf{x}}$ is minimized when it is orthogonal to the space $\mathcal{R}(\mathbf{H})$. Let

$$
\langle\mathbf{u}, \mathbf{v}\rangle_{\mathbf{W}}=\mathbf{u}^{\top} \mathbf{W} \mathbf{v},
$$

denote the weighted inner product [54]. In case of arbitrary $\mathbf{W}$ the estimation residual will be minimized when

$$
\langle\mathbf{y}-\mathbf{H} \hat{\mathbf{x}}, \mathbf{H} \hat{\mathbf{x}}\rangle_{\mathbf{W}}=\mathbf{0},
$$

is fulfilled, i.e., when $\mathbf{W}(\mathbf{y}-\mathbf{H} \hat{\mathbf{x}}) \perp \mathcal{R}(\mathbf{H})$ or equivalently $\mathbf{W}^{\frac{1}{2}}(\mathbf{y}-\mathbf{H} \hat{\mathbf{x}}) \perp \mathcal{R}\left(\mathbf{W}^{\frac{1}{2}} \mathbf{H}\right)$.

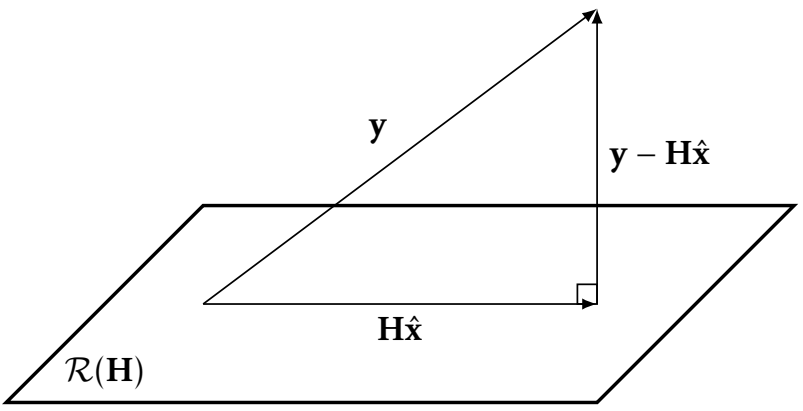

Figure 2.3: Geometrical interpretation of the WLS estimate. The WLS estimate is given by the $\hat{\mathbf{x}}$ that minimizes the residual $\mathbf{y}-\mathbf{H} \hat{\mathbf{x}}$.

Using the relationship

$$
\mathbf{H} \hat{\mathbf{x}}=\mathbf{H}\left(\mathbf{H}^{\top} \mathbf{W H}\right)^{-1} \mathbf{H}^{\top} \mathbf{W y}=\mathcal{P}_{\mathbf{H}} \mathbf{y},
$$

the projection matrix $\mathcal{P}_{\mathbf{H}}=\mathbf{H}\left(\mathbf{H}^{\top} \mathbf{W H}\right)^{-1} \mathbf{H}^{\top} \mathbf{W}$ can be defined. The matrix $\mathcal{P}_{\mathbf{H}}$ projects onto the space $\mathcal{R}(\mathbf{H})$. Similarly, the projection matrix $\mathbf{I}-\mathcal{P}_{\mathbf{H}}$ projects onto the orthogonal complement $\mathcal{R}^{\perp}(\mathbf{H})$ of $\mathcal{R}(\mathbf{H})$. Both $\mathcal{P}_{\mathbf{H}}$ and $\mathbf{I}-\mathcal{P}_{\mathbf{H}}$ are PSD [26]. The ordinary least squares (LS) estimation method is acquired by letting $\mathbf{W}$ equal the identity matrix, as was done in Figure 2.3.

\subsubsection{Properties}

The WLS estimator $\hat{\mathbf{x}}$ is linear in the data as it can be written as

$$
\hat{\mathbf{x}}=\mathbf{K y} \text {. }
$$


Since $\mathrm{E} \mathbf{v}=\mathbf{0}$ is assumed we have

$$
\mathrm{E} \hat{\mathbf{x}}=\mathrm{E}\left(\mathbf{H}^{\top} \mathbf{W H}\right)^{-1} \mathbf{H}^{\top} \mathbf{W} \mathbf{y}=\mathbf{x}
$$

i.e., the WLS estimator is unbiased. The WLS estimator which is also the BLUE is found by letting $\mathbf{W}=\mathbf{R}^{-1}$, hence yielding [26]

$$
\hat{\mathbf{x}}=\left(\mathbf{H}^{\top} \mathbf{R}^{-1} \mathbf{H}\right)^{-1} \mathbf{H}^{\top} \mathbf{R}^{-1} \mathbf{y},
$$

with covariance given by

$$
\mathbf{P}=\operatorname{cov}(\hat{\mathbf{x}})=\left(\mathbf{H}^{\top} \mathbf{R}^{-1} \mathbf{H}\right)^{-1}
$$

Since only unbiased estimators are considered it follows that $\mathbf{K}$ has to satisfy

$$
\mathbf{x}=\mathrm{E} \hat{\mathbf{x}}=\mathrm{E} \mathbf{K y}=\mathbf{K E}(\mathbf{H} \mathbf{x}+\mathbf{v})=\mathbf{K H \mathbf { x }} \Longrightarrow \mathbf{K H}=\mathbf{I},
$$

for all $\mathbf{x}$. In the Gauss-Markov theorem, it is shown that a WLS estimator with $\mathbf{W}=\mathbf{R}^{-1}$ is the BLUE, see Theorem 2.1. To be clear, the BLUE is given by

$$
\begin{aligned}
\hat{\mathbf{x}}^{\text {BLUE }} & =\left(\mathbf{H}^{\top} \mathbf{R}^{-1} \mathbf{H}\right)^{-1} \mathbf{H}^{\top} \mathbf{R}^{-1} \mathbf{y}, \\
\mathbf{P}^{\text {BLUE }} & =\left(\mathbf{H}^{\top} \mathbf{R}^{-1} \mathbf{H}\right)^{-1},
\end{aligned}
$$

which is the notation that will be used henceforth.

Theorem 2.1 (Gauss-Markov Theorem). Consider y given as

$$
\mathbf{y}=\mathbf{H x}+\mathbf{v}
$$

where $\mathbf{x}$ is the true state to be estimated, $\operatorname{rank}(\mathbf{H})=\operatorname{dim}(\mathbf{x})$ and $\mathbf{v}$ is a zero-mean random noise with covariance $\mathbf{R}=\operatorname{cov}(\mathbf{v})$. Then the best linear unbiased estimator of $\mathbf{x}$ is given by the gain $\mathbf{K}=\left(\mathbf{H}^{\top} \mathbf{R}^{-1} \mathbf{H}\right)^{-1} \mathbf{H}^{\top} \mathbf{R}^{-1}$, i.e.,

$$
\hat{\mathbf{x}}^{*}=\left(\mathbf{H}^{\top} \mathbf{R}^{-1} \mathbf{H}\right)^{-1} \mathbf{H}^{\top} \mathbf{R}^{-1} \mathbf{y} .
$$

The error covariance of $\hat{\mathbf{x}}$ is given by

$$
\mathbf{P}^{*}=\left(\mathbf{H}^{\top} \mathbf{R}^{-1} \mathbf{H}\right)^{-1} \text {. }
$$

Proof: Since $\mathbf{R}>\mathbf{0}$, it has a Cholesky decomposition $\mathbf{R}=\mathbf{L L}^{\top}$, where $\mathbf{L}$ is nonsingular [19]. Using $\mathbf{y}^{\prime}=\mathbf{L}^{-1} \mathbf{y}, \mathbf{H}^{\prime}=\mathbf{L}^{-1} \mathbf{H}$ and $\mathbf{v}^{\prime}=\mathbf{L}^{-1} \mathbf{v}$, we can instead equally well study the transformed model

$$
\mathbf{y}^{\prime}=\mathbf{H}^{\prime} \mathbf{x}+\mathbf{v}^{\prime}
$$

where $\mathbf{R}^{\prime}=\operatorname{cov}\left(\mathbf{v}^{\prime}\right)=\mathbf{I}$. In this case the WLS estimator notationally reduces to the LS estimator

$$
\hat{\mathbf{x}}^{*}=\left(\left(\mathbf{H}^{\prime}\right)^{\top} \mathbf{H}^{\prime}\right)^{-1}\left(\mathbf{H}^{\prime}\right)^{\top} \mathbf{y}^{\prime},
$$


where we now have $\operatorname{cov}\left(\hat{\mathbf{x}}^{*}\right)=\left(\left(\mathbf{H}^{\prime}\right)^{\top} \mathbf{H}^{\prime}\right)^{-1}$. Let $\hat{\mathbf{x}}=\mathbf{K} \mathbf{y}^{\prime}$ denote an arbitrary linear unbiased estimator of $\mathbf{x}$, where $\operatorname{cov}(\hat{\mathbf{x}})=\mathbf{K} \mathbf{K}^{\top}$. The unbiased constraint $\mathbf{K H}^{\prime}=\mathbf{I}$ yields

$$
\begin{aligned}
\operatorname{cov}(\hat{\mathbf{x}})-\operatorname{cov}\left(\hat{\mathbf{x}}^{*}\right) & =\mathbf{K} \mathbf{K}^{\top}-\left(\left(\mathbf{H}^{\prime}\right)^{\top} \mathbf{H}^{\prime}\right)^{-1} \\
& =\mathbf{K} \mathbf{K}^{\top}-\mathbf{K} \mathbf{H}^{\prime}\left(\left(\mathbf{H}^{\prime}\right)^{\top} \mathbf{H}^{\prime}\right)^{-1}\left(\mathbf{H}^{\prime}\right)^{\top} \mathbf{K}^{\top} \\
& =\mathbf{K}\left(\mathbf{I}-\mathbf{H}^{\prime}\left(\left(\mathbf{H}^{\prime}\right) \mathbf{H}^{\prime}\right)^{-1}\left(\mathbf{H}^{\prime}\right)^{\top}\right) \mathbf{K}^{\top} \\
& =\mathbf{K}\left(\mathbf{I}-\mathcal{P}_{\mathbf{H}^{\prime}}\right) \mathbf{K}^{\top},
\end{aligned}
$$

where the projection matrix $\mathbf{I}-\mathcal{P}_{\mathbf{H}^{\prime}}$ has been identified. Since $\mathbf{I}-\mathcal{P}_{\mathbf{H}^{\prime}} \geq \mathbf{0}$, the quadratic form above gives us $\operatorname{cov}(\hat{\mathbf{x}})-\operatorname{cov}\left(\hat{\mathbf{x}}^{*}\right) \geq \mathbf{0}$.

\subsubsection{Information Form}

In estimation problems it is often convenient to use the information form [33]. The information matrix, or simply information, $\mathcal{I}$, and the information state $\iota$ are defined as

$$
\begin{aligned}
\mathcal{I} & =\mathbf{P}^{-1}, \\
\iota & =\mathbf{P}^{-1} \hat{\mathbf{X}} .
\end{aligned}
$$

The information form has the following advantages:

1. Additive: When combining independent information $\mathcal{I}_{1}, \mathcal{I}_{2}, \ldots$ the combined information $\mathcal{I}$ is the sum $\mathcal{I}=\sum_{l} \mathcal{I}_{l}[18]$.

2. Representation of zero information: Infinite variance in any component is represented by zero information in the same direction [18].

3. Sparse: The information matrix is often sparse [63].

In information form the BLUE is given by $\hat{\mathbf{x}}^{\mathrm{BLUE}}=\mathcal{I}_{\iota}$ with

$$
\begin{aligned}
\mathcal{I} & =\mathbf{H}^{\top} \mathbf{R}^{-1} \mathbf{H}, \\
\iota & =\mathbf{H}^{\top} \mathbf{R}^{-1} \mathbf{y} .
\end{aligned}
$$

Despite the use of $\iota$ and $\mathcal{I}$ is conventional notation, to reduce the number of symbols, $\mathbf{P}^{-1} \hat{\mathbf{x}}$ and $\mathbf{P}^{-1}$ will instead be used to express the information form, with a few exceptions. Information matrices including their useful geometrical representation as ellipsoids are studied in Example 2.2 and illustrated in Figure 2.4.

\section{Example 2.2: Information Ellipses}

The two-dimensional covariance matrices

$$
\mathbf{R}_{1}=\left[\begin{array}{ll}
a & 0 \\
0 & d
\end{array}\right], \quad \mathbf{R}_{2}=\left[\begin{array}{ll}
a^{\prime} & b^{\prime} \\
b^{\prime} & d^{\prime}
\end{array}\right],
$$




\section{Covariance domain}

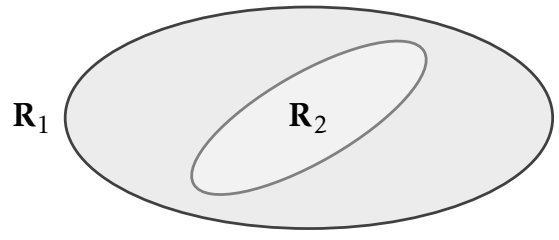

\section{Information domain}

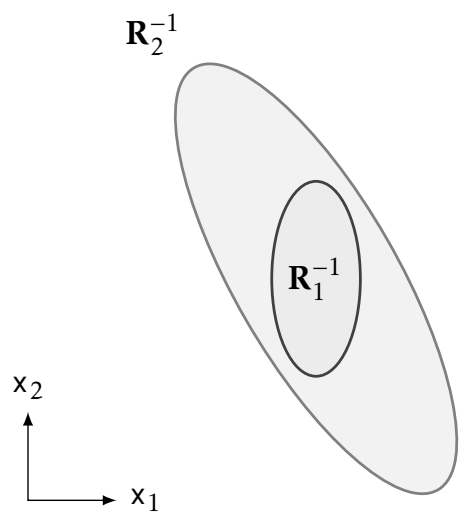

Figure 2.4: Covariance matrices illustrated as ellipses and their information counterparts. The two domains are referenced using a common frame defined by $x_{1}$ and $x_{2}$.

are given. Using the eigendecompositions $\mathbf{R}_{1}=\sum_{i=1}^{2} \lambda_{i} \mathbf{v}_{i} \mathbf{v}_{i}^{\top}$ and $\mathbf{R}_{2}=\sum_{i=1}^{2} \beta_{i} \mathbf{u}_{i} \mathbf{u}_{i}^{\top}$, the corresponding information matrices can be expressed as

$$
\mathbf{R}_{1}^{-1}=\sum_{i=1}^{2} \lambda_{i}^{-1} \mathbf{v}_{i} \mathbf{v}_{i}^{\top}, \quad \mathbf{R}_{2}^{-1}=\sum_{i=1}^{2} \beta_{i}^{-1} \mathbf{u}_{i} \mathbf{u}_{i}^{\top} .
$$

The ellipses for $\mathbf{R}_{1}$ and $\mathbf{R}_{2}$ together with their information counterparts, $\mathbf{R}_{1}^{-1}$ and $\mathbf{R}_{2}^{-1}$, are illustrated in Figure 2.4. For each ellipse, minor and major axes interchanges when shifting from the covariance domain to the information domain. In this case $\mathbf{R}_{1}$ encloses $\mathbf{R}_{2}$ in the covariance domain and hence $\mathbf{R}_{2}^{-1}$ encloses $\mathbf{R}_{1}^{-1}$ in the information domain .

\subsection{Linear Unbiased Fusion}

Fusion is a special case of estimation where estimates are combined to produce improved estimates [18]. The notation $\hat{\mathbf{z}}_{l}$ is used for partial estimates to be fused, where the covariance is given by $\mathbf{C}_{\imath}=\operatorname{cov}\left(\hat{\mathbf{z}}_{\imath}\right)$. The index $\imath$ (and $\jmath$ ) will be used introduced for numbering the partial estimates. The fused estimate is given by $\hat{\mathbf{x}}$ with the covariance given by $\mathbf{P}$. It is still assumed all estimators are unbiased.

\subsubsection{Correlated Estimates}

The covariance of an estimate $\hat{\mathbf{z}}_{l}$ is given by

$$
\mathbf{C}_{l}=\operatorname{cov}\left(\hat{\mathbf{z}}_{l}\right)=\mathrm{E}\left(\hat{\mathbf{z}}_{l}-\mathrm{E} \hat{\mathbf{z}}_{l}\right)\left(\hat{\mathbf{z}}_{l}-\mathrm{E} \hat{\mathbf{z}}_{l}\right)^{\top}=\mathrm{E}\left(\hat{\mathbf{z}}_{l}-\mathbf{H}_{l} \mathbf{x}\right)\left(\hat{\mathbf{z}}_{l}-\mathbf{H}_{l} \mathbf{x}\right)^{\top} .
$$


The cross-correlation between two estimates $\hat{\mathbf{z}}_{l}$ and $\hat{\mathbf{z}}_{j}$ is described by the crosscovariance defined as

$$
\mathbf{C}_{\imath \jmath}=\mathbf{C}_{j l}^{\top}=\operatorname{cov}\left(\hat{\mathbf{z}}_{l}, \hat{\mathbf{z}}_{j}\right)=\mathrm{E}\left(\hat{\mathbf{z}}_{l}-\mathrm{E} \hat{\mathbf{z}}_{l}\right)\left(\hat{\mathbf{z}}_{j}-\mathrm{E} \hat{\mathbf{z}}_{j}\right)^{\top}=\mathrm{E}\left(\hat{\mathbf{z}}_{l}-\mathbf{H}_{l} \mathbf{x}\right)\left(\hat{\mathbf{z}}_{j}-\mathbf{H}_{j} \mathbf{x}\right)^{\top} .
$$

Occasionally the dimensionality $n_{\imath}=\operatorname{dim}\left(\hat{\mathbf{z}}_{\imath}\right) \leq \operatorname{dim}(\mathbf{x})=n_{x}$, i.e., $\hat{\mathbf{z}}_{\imath}$ might be an estimate of only parts of $\hat{\mathbf{x}}$. The estimate $\hat{\mathbf{z}}_{l}$ is modeled as

$$
\hat{\mathbf{z}}_{\imath}=\mathbf{H}_{\imath} \mathbf{x}+\mathbf{e}_{\imath}
$$

where $\mathbf{e}_{\imath}$ is zero-mean noise. The model in (2.40) is equivalent to (2.1) except that the data $\mathbf{y}$ now has been replaced by the estimate $\hat{\mathbf{z}}_{\imath}, \mathbf{v}$ has been replaced by $\mathbf{e}_{\imath}$, and subscripted index $\imath$ has been introduced.

Denoting $\mathbf{x} \in \mathcal{X}$ and $\mathbf{H}_{\imath} \mathbf{x} \in \mathcal{Z}_{l}$, the matrix $\mathbf{H}_{\imath}$ may be viewed as the mapping

$$
\mathbf{H}_{\imath}: \mathcal{X} \longrightarrow \mathcal{Z}_{\imath}
$$

If $n_{l}<n_{x}$ then $\hat{\mathbf{z}}_{l}$ is a strictly partial estimate of $\mathbf{x}$. If $n_{l}=n_{x}$ then $\hat{\mathbf{z}}_{l}$ is a full estimate of $\mathbf{x}$. In this scope $\hat{\mathbf{z}}_{l}$ will simply be referred to as a partial estimate, where partial estimates comprise both strictly partial estimates and full estimates. For partial estimates it is true that

$$
\operatorname{rank}\left(\mathbf{H}_{\imath}\right) \leq n_{x}
$$

with equality if $\mathcal{Z}_{\imath}$ and $\mathcal{X}$ represent the same state space. The assumption $\mathrm{E} \mathbf{e}_{\imath}=\mathbf{0}$ yields

$$
\mathrm{E} \hat{\mathbf{z}}_{l}=\mathrm{E}\left(\mathbf{H}_{l} \mathbf{x}+\mathbf{e}_{\imath}\right)=\mathbf{H}_{l} \mathbf{x},
$$

i.e., the estimate $\hat{\mathbf{z}}_{l}$ is unbiased and $\mathbf{H}_{l} \mathbf{x}$ is the true state in $\mathcal{Z}_{l}$.

Often it is beneficial to express multiple estimates and models jointly according to

$$
\begin{aligned}
\hat{\mathbf{z}}_{J} & =\operatorname{col}\left(\hat{\mathbf{z}}_{1}, \ldots, \hat{\mathbf{z}}_{N}\right), \\
\mathbf{H}_{J} & =\operatorname{col}\left(\mathbf{H}_{1}, \ldots, \mathbf{H}_{N}\right), \\
\mathbf{e}_{J} & =\operatorname{col}\left(\mathbf{e}_{1}, \ldots, \mathbf{e}_{N}\right),
\end{aligned}
$$

where $\operatorname{col}(\cdot)$ is an operator which stacks the input arguments as a column and $N$ is the number of estimates to be stacked. Using (2.44) it is possible to define the linear model on joint estimate form as

$$
\hat{\mathbf{z}}_{J}=\mathbf{H}_{J} \mathbf{x}+\mathbf{e}_{J}
$$

where the joint covariance $\mathbf{C}_{J}=\operatorname{cov}\left(\hat{\mathbf{z}}_{J}\right)$ is given by

$$
\mathbf{C}_{J}=\left[\begin{array}{cccc}
\mathbf{C}_{1} & \mathbf{C}_{12} & \ldots & \mathbf{C}_{1 N} \\
\mathbf{C}_{21} & \mathbf{C}_{2} & \ldots & \mathbf{C}_{2 N} \\
\vdots & \vdots & \ddots & \vdots \\
\mathbf{C}_{N 1} & \mathbf{C}_{N 2} & \ldots & \mathbf{C}_{N}
\end{array}\right]
$$


Now the linear fusion rule can be written compactly as

$$
\hat{\mathbf{x}}=\mathbf{K}_{J} \hat{\mathbf{z}}_{J}
$$

where

$$
\mathbf{K}_{J}=\left[\begin{array}{lll}
\mathbf{K}_{1} & \ldots & \mathbf{K}_{N}
\end{array}\right]
$$

is the joint fusion gain.

\subsubsection{Fusion Under Known Cross-Correlations}

Fusion of two cross-correlated full estimates can be performed using the BarShalom-Campo (BSC) formula [3]. The BSC formula is provided in Algorithm 2.1, where it is implicitly assumed $\mathbf{H}_{1}=\mathbf{H}_{2}=\mathbf{I}$.

The BSC formula can be derived as follows. The unbiased constraint (2.34) implies

$$
\hat{\mathbf{x}}=\mathbf{K}_{1} \hat{\mathbf{z}}_{1}+\mathbf{K}_{2} \hat{\mathbf{z}}_{2}=\mathbf{K}_{1} \hat{\mathbf{z}}_{1}+\left(\mathbf{I}-\mathbf{K}_{1}\right) \hat{\mathbf{z}}_{2}=\mathbf{K} \hat{\mathbf{z}}_{1}+(\mathbf{I}-\mathbf{K}) \hat{\mathbf{z}}_{2},
$$

where $\mathbf{K}_{1}=\mathbf{K}$ and $\mathbf{K}_{2}=\mathbf{I}-\mathbf{K}$. Completion of squares yields

$$
\begin{aligned}
\mathbf{P}= & {\left[\begin{array}{ll}
\mathbf{K} & (\mathbf{I}-\mathbf{K})
\end{array}\right]\left[\begin{array}{cc}
\mathbf{C}_{1} & \mathbf{C}_{12} \\
\mathbf{C}_{21} & \mathbf{C}_{2}
\end{array}\right]\left[\begin{array}{c}
\mathbf{K}^{\top} \\
(\mathbf{I}-\mathbf{K})^{\top}
\end{array}\right] } \\
= & \mathbf{K} \mathbf{C}_{1} \mathbf{K}^{\top}+\mathbf{C}_{21} \mathbf{K}^{\top}-\mathbf{K} \mathbf{C}_{21} \mathbf{K}^{\top}+\mathbf{K} \mathbf{C}_{12}-\mathbf{K} \mathbf{C}_{12} \mathbf{K}^{\top}+\mathbf{C}_{2}-\mathbf{K} \mathbf{C}_{2}-\mathbf{C}_{2} \mathbf{K}^{\top}+\mathbf{K} \mathbf{C}_{2} \mathbf{K}^{\top} \\
= & \mathbf{C}_{2}-\mathbf{K}\left(\mathbf{C}_{2}-\mathbf{C}_{12}\right)+\left(\mathbf{C}_{2}-\mathbf{C}_{21}\right) \mathbf{K}^{\top}+\mathbf{K}\left(\mathbf{C}_{1}+\mathbf{C}_{2}-\mathbf{C}_{12}-\mathbf{C}_{21}\right) \mathbf{K}^{\top} \\
= & \mathbf{C}_{2}-\left(\mathbf{C}_{2}-\mathbf{C}_{21}\right)\left(\mathbf{C}_{1}+\mathbf{C}_{2}-\mathbf{C}_{12}-\mathbf{C}_{21}\right)^{-1}\left(\mathbf{C}_{2}-\mathbf{C}_{21}\right)^{\top} \\
& +\left(\mathbf{K}-\left(\mathbf{C}_{2}-\mathbf{C}_{21}\right)\left(\mathbf{C}_{1}+\mathbf{C}_{2}-\mathbf{C}_{12}-\mathbf{C}_{21}\right)^{-1}\right)\left(\mathbf{C}_{1}+\mathbf{C}_{2}-\mathbf{C}_{12}-\mathbf{C}_{21}\right)(\cdot)^{\top},
\end{aligned}
$$

where $\mathbf{A B}(\cdot)^{\top}=\mathbf{A} \mathbf{B} \mathbf{A}^{\top}$. Since only the last term in the last step of (2.50) contains $\mathbf{K}$ and also is quadratic, $\mathbf{P}$ will be minimized when

$$
\mathbf{K}=\left(\mathbf{C}_{2}-\mathbf{C}_{21}\right)\left(\mathbf{C}_{1}+\mathbf{C}_{2}-\mathbf{C}_{12}-\mathbf{C}_{21}\right)^{-1} \text {. }
$$

By putting $\mathbf{K}_{1}=\mathbf{K}$ and $\mathbf{K}_{2}=\mathbf{I}-\mathbf{K}$ the formula in Algorithm 2.1 is recovered.

A fusion method which is equivalent to the BSC formula is a direct application of the WLS estimator proposed earlier in Section 2.3, i.e., the BLUE. The estimate and covariance of the BLUE derived from the joint estimate are given by

$$
\begin{aligned}
\hat{\mathbf{x}}^{\mathrm{BLUE}} & =\left(\mathbf{H}_{J}^{\top} \mathbf{C}_{J}^{-1} \mathbf{H}_{J}\right)^{-1} \mathbf{H}_{J}^{\top} \mathbf{C}_{J}^{-1} \hat{\mathbf{z}}_{J}, \\
\mathbf{P}^{\mathrm{BLUE}} & =\left(\mathbf{H}_{J}^{\top} \mathbf{C}_{J}^{-1} \mathbf{H}_{J}\right)^{-1},
\end{aligned}
$$

which is valid for arbitrary $N$ and where it is assumed that $\operatorname{rank}\left(\mathbf{H}_{J}\right)=n_{x}$. The corresponding information form of (2.54) is given by

$$
\begin{aligned}
\left(\mathbf{P}^{\mathrm{BLUE}}\right)^{-1} & =\mathbf{H}_{J}^{\top} \mathbf{C}_{J}^{-1} \mathbf{H}_{J}, \\
\left(\mathbf{P}^{\mathrm{BLUE}}\right)^{-1} \hat{\mathbf{x}}^{\mathrm{BLUE}} & =\mathbf{H}_{J}^{\top} \mathbf{C}_{J}^{-1} \hat{\mathbf{z}}_{J} .
\end{aligned}
$$

As a demonstration of the fusion of two correlated estimates using the BLUE Example 2.3 is provided below. An illustration of Example 2.3 is given in Figure 2.5 . 


\section{Algorithm 2.1: Bar-Shalom-Campo Formula}

Input: $\hat{\mathbf{z}}_{1}, \hat{\mathbf{z}}_{2}, \mathrm{C}_{1}, \mathrm{C}_{2}, \mathrm{C}_{12}, \mathrm{C}_{21}$

The estimates are fused according to

$$
\begin{aligned}
& \hat{\mathbf{x}}=\mathbf{K}_{1} \hat{\mathbf{z}}_{1}+\mathbf{K}_{2} \hat{\mathbf{z}}_{2}, \\
& \mathbf{P}=\mathbf{C}_{1}-\mathbf{K}_{2}\left(\mathbf{C}_{1}+\mathbf{C}_{2}-\mathbf{C}_{12}-\mathbf{C}_{21}\right) \mathbf{K}_{2}^{\top},
\end{aligned}
$$

where the fusion gains are given by

$$
\begin{aligned}
& \mathbf{K}_{1}=\left(\mathbf{C}_{2}-\mathbf{C}_{21}\right)\left(\mathbf{C}_{1}+\mathbf{C}_{2}-\mathbf{C}_{12}-\mathbf{C}_{21}\right)^{-1}, \\
& \mathbf{K}_{2}=\left(\mathbf{C}_{1}-\mathbf{C}_{12}\right)\left(\mathbf{C}_{1}+\mathbf{C}_{2}-\mathbf{C}_{12}-\mathbf{C}_{21}\right)^{-1} .
\end{aligned}
$$

Output: $\hat{\mathbf{x}}, \mathbf{P}$

\section{Example 2.3: Fusing Correlated Estimates}

Consider fusion of $\hat{\mathbf{z}}_{1}=\left[\begin{array}{ll}1 & 0\end{array}\right]^{\top}$ and $\hat{\mathbf{z}}_{2}=\left[\begin{array}{ll}0 & 1\end{array}\right]^{\top}$ where $\mathbf{H}_{1}=\mathbf{H}_{2}=\mathbf{I}$ and

$$
\mathbf{C}_{1}=\operatorname{cov}\left(\hat{\mathbf{z}}_{1}\right)=\left[\begin{array}{ll}
4 & 1 \\
1 & 2
\end{array}\right], \quad \mathbf{C}_{2}=\operatorname{cov}\left(\hat{\mathbf{z}}_{2}\right)=\left[\begin{array}{cc}
2 & -1 \\
-1 & 4
\end{array}\right]
$$

The cross-covariance is given by

$$
\mathrm{C}_{12}=\operatorname{cov}\left(\hat{\mathbf{z}}_{1}, \hat{\mathbf{z}}_{2}\right)=\left[\begin{array}{ll}
2 & 0 \\
0 & 2
\end{array}\right]
$$

On joint form the input parameters are given by

$$
\hat{\mathbf{z}}_{J}=\left[\begin{array}{l}
1 \\
0 \\
0 \\
1
\end{array}\right], \quad \mathbf{C}_{J}=\left[\begin{array}{cccc}
4 & 1 & 2 & 0 \\
1 & 2 & 0 & 2 \\
2 & 0 & 2 & -1 \\
0 & 2 & -1 & 4
\end{array}\right] \text {, }
$$

and $\mathbf{H}_{J}=\left[\begin{array}{ll}\mathbf{I} & \mathbf{I}\end{array}\right]^{\top}$. The formula in (2.54) yields

$$
\hat{\mathbf{x}}^{\text {BLUE }}=\frac{1}{2}\left[\begin{array}{c}
1 \\
-1
\end{array}\right], \quad \mathbf{P}^{\text {BLUE }}=\frac{3}{2}\left[\begin{array}{ll}
1 & 0 \\
0 & 1
\end{array}\right]
$$

The results are shown in Figure 2.5 where also the true state $\mathbf{x}=\left[\begin{array}{ll}0 & 0\end{array}\right]^{\top}$ is provided. 
Input estimates

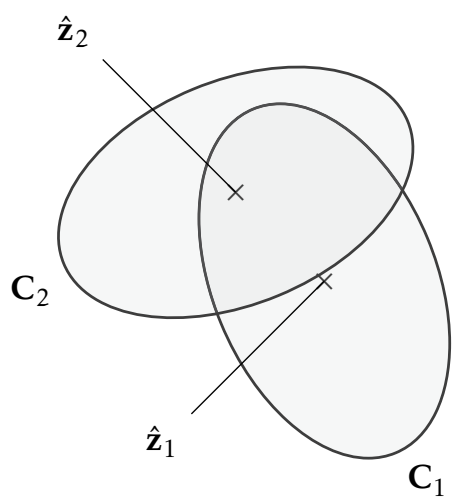

\section{Fusion results}

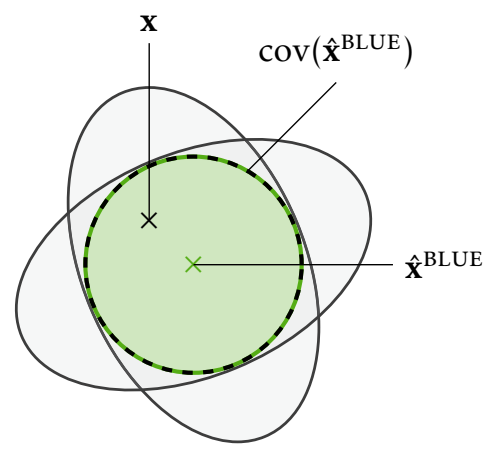

Figure 2.5: Fusing correlated estimates using the BLUE. On the left side each ellipse is centered about the corresponding estimate. On the right side all ellipses are centered about $\hat{\mathbf{x}}^{\mathrm{BLUE}}$.

\subsubsection{Fusion Under Zero Cross-Correlations}

If uncorrelated estimates can be assumed, i.e., if

$$
\mathbf{C}_{J}=\left[\begin{array}{cccc}
\mathbf{C}_{1} & \mathbf{0} & \ldots & \mathbf{0} \\
\mathbf{0} & \mathbf{C}_{2} & \ddots & \vdots \\
\vdots & \ddots & \ddots & \mathbf{0} \\
\mathbf{0} & \ldots & \mathbf{0} & \mathbf{C}_{N}
\end{array}\right]
$$

is satisfied then $(2.55)$ reduces to

$$
\begin{aligned}
\mathbf{P}^{-1} & =\sum_{\imath=1}^{N} \mathbf{H}_{\imath}^{\top} \mathbf{C}_{l}^{-1} \mathbf{H}_{\imath}, \\
\mathbf{P}^{-1} \hat{\mathbf{x}} & =\sum_{\imath=1}^{N} \mathbf{H}_{\imath}^{\top} \mathbf{C}_{\imath}^{-1} \hat{\mathbf{z}}_{\imath},
\end{aligned}
$$

which is the information form of the sensor fusion formula [18]. An estimate calculated using (2.57) is in the following regarded as a naïvely fused estimate since all input estimates are assumed uncorrelated. 


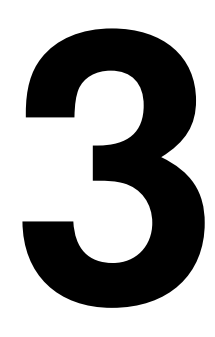

\title{
Conservative Linear Unbiased Estimation
}

\begin{abstract}
COnSERVATIVE estimation PROBlems are encountered in setups where some knowledge about the covariance $\mathbf{R}=\operatorname{cov}(\mathbf{y})$ is unavailable, e.g., in decentralized sensor networks. As in the previous chapter, the linear model $\mathbf{y}=\mathbf{H x}+\mathbf{v}$ is assumed for the data to derive an estimate $\hat{\mathbf{x}}$ from, where $\mathbf{x}$ is the true state, $\mathrm{E} \mathbf{v}=\mathbf{0}$ and $\operatorname{rank}(\mathbf{H})=n_{x}$.

The partial knowledge about $\mathbf{R}$ is modeled using a set $\mathfrak{K} \subseteq \mathfrak{S}^{++}$which contain all matrices that $\mathbf{R}$ may be equal to. We then say $\mathbf{R} \in \mathcal{R}$. This means that we need to distinguish between the true covariance $\operatorname{cov}(\hat{\mathbf{x}})$ and the covariance $\mathbf{P}$ calculated by the estimator since these two will generally not be equal in this class of problems, something that is not encountered in the classical estimation problem studied in Chapter 2. Essentially, conservative estimation can be summarized by two partly conflicting objectives:
\end{abstract}

1. Ensure $\mathbf{P} \geq \operatorname{cov}(\hat{\mathbf{x}})$ given that $\mathbf{R} \in \mathfrak{K}$ is the only knowledge about $\mathbf{R}$.

2. Minimize $\mathbf{P}$ under the constraint $\mathbf{P} \geq \operatorname{cov}(\hat{\mathbf{x}})$.

That is, we do not give up just because of our lack of knowledge about $\mathbf{R}$, but instead conservatively try to do our best despite only knowing $\mathbf{R} \in \mathfrak{R}$.

In this chapter the general conservative linear unbiased estimation problem is formalized, where $\mathbf{y}$ is the input to the estimator, but where it is only known that $\mathbf{R} \in \mathfrak{K}$. Only estimators that are linear and unbiased will be considered. Furthermore, the conservative linear unbiased estimator (CLUE) and optimal CLUEs are defined. At last, the conservative Gauss-Markov theorem is given.

\section{Contributions}

Several contributions are included in this chapter, dispersed in the text: 
General. A number of examples are provided that exploit different aspects of the conservative estimation problem.

CLUE. The conservative linear unbiased estimator is given a formal definition (Definition 3.1). This particular estimator is not only useful within linear estimation problems where $\mathbf{R}$ is partly known, but also as a reference estimator for related nonlinear estimation problems.

Optimal CLUE. Two different optimal CLUEs are given; (1) the best CLUE, which should be interpreted as the unrestrictedly optimal CLUE, and (2) the restricted best CLUE which is, as the name suggests, optimal under certain restrictions, Definition 3.2 and 3.4, respectively.

Conservative Gauss-Markov theorem. A conservative analogue of the GaussMarkov theorem is proposed in Theorem 3.2. The conservative Gauss-Markov theorem is helpful when it comes to finding a restricted best CLUE.

\subsection{Problem Formulation}

Prior to turning to the main problem considered in this chapter, the (classical) linear unbiased estimation problem is revisited. Then a stepwise transition is made until we arrive at the conservative linear unbiased estimation problem. The assumptions $\hat{\mathbf{x}}=\mathbf{K y}$ and $\mathrm{E} \hat{\mathbf{x}}=\mathbf{x}$ imply $\mathbf{K H}=\mathbf{I}$.

\subsubsection{The Linear Unbiased Estimation Problem Revisited}

For a linear unbiased estimator the estimate is calculated as $\hat{\mathbf{x}}=\mathbf{K y}$ where $E \hat{\mathbf{x}}=\mathbf{x}$ and

$$
\operatorname{cov}(\hat{\mathbf{x}})=\operatorname{cov}(\mathbf{K y})=\mathbf{K} \operatorname{cov}(\mathbf{y}) \mathbf{K}^{\top}=\mathbf{K} \mathbf{R} \mathbf{K}^{\top},
$$

which is in accordance with (2.14). The overall goal is to find the optimal estimate, i.e., the estimate $\hat{\mathbf{x}}$ having the smallest covariance $\mathbf{P}$.

In classical linear unbiased estimation we have $\mathcal{K}=\{\mathbf{R}\}$ and hence the problem can be stated as

$$
\begin{array}{ll}
\mathbf{K}^{*}=\underset{\mathbf{K}}{\arg \min } & \mathbf{P} \\
\text { subject to } & \mathbf{K H}=\mathbf{I} \\
& \mathbf{P}=\mathbf{K} \mathbf{R} \mathbf{K}^{\top}=\operatorname{cov}(\mathbf{K y}),
\end{array}
$$

where $\mathbf{K}^{*}$ is the optimal gain. The operator $\arg \min _{\mathbf{K}} \mathbf{P}$ where the target $\mathbf{P}$ is a matrix variable means minimization of $\mathbf{P}$ in the PSD sense w.r.t. the argument $\mathbf{K}$. This means that we seek $\mathbf{K}^{*}$ such that

$$
\mathbf{K R K}^{\top} \geq \mathbf{K}^{*} \mathbf{R}\left(\mathbf{K}^{*}\right)^{\top},
$$

holds for all $\mathbf{K}$ satisfying $E \mathbf{K y}=\mathbf{x}$ or equivalently $\mathbf{K H}=\mathbf{I}$. It is important to note that for general problems where a smallest matrix is sought, like in (3.2), 
a solution does not exist since we typically end up with comparing incomparable matrices. However, for the particular problem formulated in (3.2) a solution exists, see below.

The optimization problem of (3.2) can be formulated differently such that the transition to the conservative estimation problem becomes somewhat smoother. An equivalent problem, in the sense that it has the same solution, is given by

$$
\begin{array}{ll}
\mathbf{K}^{*}, \mathbf{P}^{*}=\underset{\mathbf{K}, \mathbf{P}}{\arg \min } & \mathbf{P} \\
\text { subject to } & \mathbf{K H}=\mathbf{I} \\
& \mathbf{P} \geq \mathbf{K} \mathbf{R}^{\prime} \mathbf{K}^{\top}, \forall \mathbf{R}^{\prime} \in \mathfrak{K}=\{\mathbf{R}\} \\
& \mathbf{P} \in \mathfrak{S}^{++},
\end{array}
$$

where the optimization is now over two arguments, $\mathbf{K}$ and $\mathbf{P}$. Moreover, $\mathbf{P}^{*}$ is the optimal covariance and the equality $\mathbf{P}=\mathbf{K} \mathbf{R} \mathbf{K}^{\top}$ has now been replaced by $\mathbf{P} \geq \mathbf{K} \mathbf{R} \mathbf{K}^{\top}$, i.e., a condition that constraints $\mathbf{P}$ being larger than $\mathbf{K R K}^{\top}$ in the PSD sense. Since $\mathcal{R}=\{\mathbf{R}\}$ the solution to (3.4) is exactly the same as the solution to (3.2).

Theorem 2.1 tells $u$ s that if $\mathbf{y}=\mathbf{H x}+\mathbf{v}$ with $\mathbf{K H}=\mathbf{I}$, then the BLUE is given by the WLS estimator with weight matrix $\mathbf{W}=\mathbf{R}^{-1}$, i.e.,

$$
\begin{aligned}
\hat{\mathbf{x}}^{\mathrm{BLUE}} & =\left(\mathbf{H}^{\top} \mathbf{R}^{-1} \mathbf{H}\right)^{-1} \mathbf{H}^{\top} \mathbf{R}^{-1} \mathbf{y}, \\
\mathbf{P}^{\mathrm{BLUE}} & =\left(\mathbf{H}^{\top} \mathbf{R}^{-1} \mathbf{H}\right)^{-1} .
\end{aligned}
$$

\subsubsection{The Conservative Linear Unbiased Estimation Problem}

Before digging into the problem we want to solve, the following example is considered. Let $\mathcal{K}=\left\{\mathbf{R}_{1}^{\prime}, \mathbf{R}_{2}^{\prime}\right\}$ where

$$
\mathbf{R}_{1}^{\prime}=\left[\begin{array}{cccc}
1 & 0 & 0 & 0 \\
0 & 4 & 0 & 0 \\
0 & 0 & 4 & 0 \\
0 & 0 & 0 & 1
\end{array}\right], \quad \mathbf{R}_{2}^{\prime}=\left[\begin{array}{llll}
1 & 0 & 1 & 0 \\
0 & 4 & 0 & 1 \\
1 & 0 & 4 & 0 \\
0 & 1 & 0 & 1
\end{array}\right]
$$

Let $\mathbf{R}=\mathbf{R}_{2}^{\prime}$ but assume the estimator merely knows that $\mathbf{R} \in \mathcal{R}$. Assume that the only allowed gains are

$$
\begin{aligned}
\mathbf{K}_{1} & =\left(\mathbf{H}^{\top}\left(\mathbf{R}_{1}^{\prime}\right)^{-1} \mathbf{H}\right)^{-1} \mathbf{H}^{\top}\left(\mathbf{R}_{1}^{\prime}\right)^{-1}=\left[\begin{array}{cccc}
0.8 & 0 & 0.2 & 0 \\
0 & 0.2 & 0 & 0.8
\end{array}\right], \\
\mathbf{K}_{2} & =\left(\mathbf{H}^{\top}\left(\mathbf{R}_{2}^{\prime}\right)^{-1} \mathbf{H}\right)^{-1} \mathbf{H}^{\top}\left(\mathbf{R}_{2}^{\prime}\right)^{-1}=\left[\begin{array}{llll}
1 & 0 & 0 & 0 \\
0 & 0 & 0 & 1
\end{array}\right],
\end{aligned}
$$

where $\mathbf{H}=\left[\begin{array}{ll}\mathbf{I} & \mathbf{I}\end{array}\right]^{\top}$. If $\mathbf{R}=\mathbf{R}_{1}^{\prime}$ then $\mathbf{K}_{1}$ is the BLUE gain and if $\mathbf{R}=\mathbf{R}_{2}^{\prime}$ then $\mathbf{K}_{2}$ is the BLUE gain. Consider one gain at a time. If $\mathbf{K}_{1}$ is used there are two 
possibilities for the true covariance $\operatorname{cov}\left(\mathbf{K}_{1} \mathbf{y}\right)$ and thus a conservative covariance $\mathbf{P}$ has to simultaneously satisfy

$$
\mathbf{P} \geq \mathbf{K}_{1} \mathbf{R}_{1}^{\prime} \mathbf{K}_{1}^{\top}=0.8\left[\begin{array}{ll}
1 & 0 \\
0 & 1
\end{array}\right], \quad \mathbf{P} \geq \mathbf{K}_{1} \mathbf{R}_{2}^{\prime} \mathbf{K}_{1}^{\top}=1.12\left[\begin{array}{ll}
1 & 0 \\
0 & 1
\end{array}\right] .
$$

Similarly, if $\mathbf{K}_{2}$ is used then we must have

$$
\mathbf{P} \geq \mathbf{K}_{2} \mathbf{R}_{1}^{\prime} \mathbf{K}_{2}^{\top}=\left[\begin{array}{ll}
1 & 0 \\
0 & 1
\end{array}\right], \quad \mathbf{P} \geq \mathbf{K}_{2} \mathbf{R}_{2}^{\prime} \mathbf{K}_{2}^{\top}=\left[\begin{array}{ll}
1 & 0 \\
0 & 1
\end{array}\right] .
$$

The covariance $\mathbf{P}$ is supposed to satisfy (3.8) or (3.9), i.e., $\mathbf{P} \geq \operatorname{cov}(\mathbf{K y})$ for the chosen gain $\mathbf{K}$. The minimum $\mathbf{P}$ that satisfies the requirements in (3.8) is $\mathbf{P}=$ $1.12 \mathbf{I}$ and the minimum $\mathbf{P}$ that satisfies the requirements in (3.9) is $\mathbf{P}=\mathbf{I}$. Hence we conclude that the optimal solution, given $\mathfrak{K}$ and that the only allowed gains are $\mathbf{K}_{1}$ and $\mathbf{K}_{2}$, is given by

$$
\hat{\mathbf{x}}^{*}=\mathbf{K}^{*} \mathbf{y}, \quad \mathbf{P}^{*}=\left[\begin{array}{ll}
1 & 0 \\
0 & 1
\end{array}\right],
$$

where $\mathbf{K}^{*}=\mathbf{K}_{2}$. Since $\mathbf{R}=\mathbf{R}_{2}^{\prime}$ the true covariance of $\hat{\mathbf{x}}^{*}=\mathbf{K}^{*} \mathbf{y}$ is given by

$$
\operatorname{cov}\left(\mathbf{K}^{*} \mathbf{y}\right)=\mathbf{K}^{*} \mathbf{R}\left(\mathbf{K}^{*}\right)^{\top}=\left[\begin{array}{ll}
1 & 0 \\
0 & 1
\end{array}\right]
$$

which is equal to $\mathbf{P}^{*}$. In general there will be infinitely many combinations of each of $\mathbf{K}$ and $\mathbf{R}^{\prime}$. Figure 3.1 illustrates the scenario just discussed. We now return to the main problem.

In conservative linear unbiased estimation the set $\mathbb{R}$ is not the singleton $\{\mathbf{R}\}$ as in its classical counterpart. Rather $\mathcal{R}$ is a set spanning an infinite number of covariance matrices. The fact that $k \neq\{\mathbf{R}\}$ is the root of all evil in conservative estimation. Recalling the objectives stated in the preamble and the model $\mathbf{y}=$ $\mathbf{H x}+\mathbf{v}$, the conservative linear unbiased estimation problem can be stated as

$$
\begin{array}{cl}
\mathbf{K}^{*}, \mathbf{P}^{*}=\underset{\mathbf{K}, \mathbf{P}}{\arg \min } & \mathbf{P} \\
\text { subject to } & \mathbf{K H}=\mathbf{I} \\
& \mathbf{P} \geq \mathbf{K R}^{\prime} \mathbf{K}^{\top}, \forall \mathbf{R}^{\prime} \in \mathcal{K} \\
& \mathbf{P} \in \mathfrak{S}^{++},
\end{array}
$$

where $\mathbf{P}$ now is an overestimate of the true covariance $\operatorname{cov}(\mathbf{K y})$, and $\mathbf{P}^{*}$ resembles the optimal upper bound of $\operatorname{cov}\left(\mathbf{K}^{*} \mathbf{y}\right)$. An upper bound will in the following be referred to as a conservative bound. Whenever necessary for clarification, the notation $\overline{\mathbf{P}}$ will be used for a conservative bound of $\operatorname{cov}(\hat{\mathbf{x}})$, instead of $\mathbf{P}$. The smallest conservative bound is denoted $\overline{\mathbf{P}}^{*}$.

The optimization problem of (3.12) can conceptually be understood as follows: (1) Consider one gain matrix $\mathbf{K}_{i}$ at a time, and find the minimum matrix $\mathbf{P}_{i}^{*}$ 


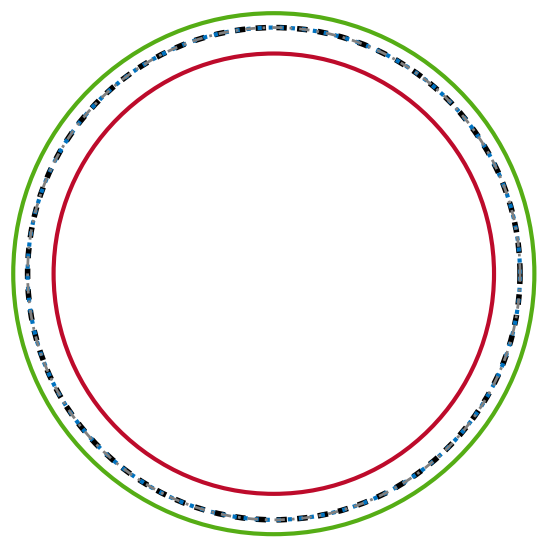

$--\operatorname{cov}\left(\mathbf{K}^{*} \mathbf{y}\right)$

$-\mathbf{K}_{1} \mathbf{R}_{1}^{\prime} \mathbf{K}_{1}^{\top}$

$-\mathbf{K}_{1} \mathbf{R}_{2}^{\prime} \mathbf{K}_{1}^{\top}$

$\cdots \mathbf{K}_{2} \mathbf{R}_{1}^{\prime} \mathbf{K}_{2}^{\top}$

$\cdots \mathbf{K}_{2} \mathbf{R}_{2}^{\prime} \mathbf{K}_{2}^{\top}$

Figure 3.1: A case where the optimal conservative estimator is sought with $\mathcal{R}=\left\{\mathbf{R}_{1}^{\prime}, \mathbf{R}_{2}^{\prime}\right\}$ and only two gain matrices $\mathbf{K}_{1}$ and $\mathbf{K}_{2}$ are considered. The matrix $\operatorname{cov}\left(\mathbf{K}^{*} \mathbf{y}\right)$ is the true covariance of the optimal estimate.

for which $\mathbf{P}_{i} \geq \mathbf{K}_{i} \mathbf{R}^{\prime} \mathbf{K}_{i}^{\top}, \forall \mathbf{R}^{\prime} \in \mathcal{R}$. (2) Add the pair $\mathbf{K}_{i}, \mathbf{P}_{i}^{*}$ as an element to the set $\mathfrak{K}$. (3) Repeat (1)-(2) for all valid $\mathbf{K}_{i}$. (4) Find the minimum element of $\mathfrak{K}$ w.r.t. $\mathbf{P}_{i}^{*}$ in the PSD sense, i.e., $\mathbf{K}^{*}, \mathbf{P}^{*}=\min \mathfrak{K}$.

Since the minimization criterion of (3.12) relies on being able to compare matrices and not all pairs of matrices are comparable (see Section 2.1.2), the criterion in (3.12) generally needs to be relaxed. One obvious way is to instead consider a loss function $J(\cdot)$ which has a scalar value as output, i.e., consider minimization of $J(\mathbf{P})$ instead of minimization of $\mathbf{P}$. We will return to this topic in Section 3.4.2.

\subsubsection{Simplified Problem}

In (3.12) there is no rule for how to calculate P. Compare this to (3.2) where $\mathbf{P}=\mathbf{K R K}^{\top}$ which follows the transformation rule of (3.1), viz.,

$$
\mathbf{y} \longrightarrow \mathrm{Ky} \Longrightarrow \mathbf{R} \longrightarrow \mathrm{KRK}^{\top} \text {. }
$$

In Chapter 2 we saw that the WLS estimator in (3.5) is the BLUE if $\mathbf{R}$ is known. What we want to do here is to imitate the particular structure of (3.13) and use it within the conservative estimation problem. Therefore, the following simplification will be made, which basically means that our search for the optimal conservative linear unbiased estimator becomes slightly restricted.

The conservative bounds on $\operatorname{cov}(\hat{\mathbf{x}})$ are given on the form $\mathbf{P}=\mathbf{K} \overline{\mathbf{R}} \mathbf{K}^{\top}$ where $\overline{\mathbf{R}} \in$ $\overline{\mathfrak{R}}$ is a conservative bound of $\mathbf{R}$. The set $\overline{\mathfrak{R}}$ includes all $\overline{\mathbf{R}}$ for which $\overline{\mathbf{R}} \geq \mathbf{R}^{\prime}, \forall \mathbf{R}^{\prime} \in$ $\mathfrak{R}$ holds. As will be seen later in the conservative Gauss-Markov theorem the smallest such $\mathbf{P}=\mathbf{K} \overline{\mathbf{R}} \mathbf{K}^{\top}$ is given by $\overline{\mathbf{R}}$ and $\mathbf{K}$ according to

$$
\overline{\mathbf{R}}^{*}=\min \overline{\mathcal{R}}, \quad \quad \mathbf{K}^{*}=\left(\mathbf{H}^{\top}\left(\overline{\mathbf{R}}^{*}\right)^{-1} \mathbf{H}\right) \mathbf{H}^{\top}\left(\overline{\mathbf{R}}^{*}\right)^{-1},
$$


where min $\overline{\mathcal{K}}$ refers to the smallest element in $\overline{\mathcal{K}}$ (smallest in the PSD sense).

If all of the diagonal blocks of $\mathbf{R}$ are known while all the off-diagonal blocks are unknown, then we will restrict all $\overline{\mathbf{R}}$ to being block diagonal where each diagonal block is of the same size as the corresponding diagonal blocks of $\mathbf{R}$. This assumption is used in Chapter 4.

It must be emphasized that under the previously stated simplification we do not necessarily solve (3.12).

\subsubsection{Problem Properties}

The data input to the estimate is $\mathbf{y}=\operatorname{col}\left(\mathbf{y}_{1}, \ldots, \mathbf{y}_{2}\right)$ with covariance

$$
\mathbf{R}=\left[\begin{array}{cccc}
\mathbf{R}_{1} & \mathbf{R}_{12} & \ldots & \mathbf{R}_{1 N} \\
\mathbf{R}_{21} & \mathbf{R}_{2} & \ldots & \mathbf{R}_{2 N} \\
\vdots & \vdots & \ddots & \vdots \\
\mathbf{R}_{N 1} & \mathbf{R}_{N 2} & \ldots & \mathbf{R}_{N}
\end{array}\right]
$$

Assume $\operatorname{dim}\left(\mathbf{y}_{\imath}\right)=\operatorname{dim}(\hat{\mathbf{x}}), \forall \imath$. The following can be said about the estimate $\hat{\mathbf{x}}$ to get an intuition for the problem encountered herein:

- If $\mathcal{R}=\{\mathbf{R}\}$ then $\mathbf{P}=\left(\mathbf{H}^{\top} \mathbf{R}^{-1} \mathbf{H}\right)^{-1}$ yields an optimally fused estimate, where (2.54) has been used.

- In case all off-diagonal blocks of $\mathbf{R}$ are zero then the naïve estimation rule of (2.57) provides an optimal estimate $\hat{\mathbf{x}}$ where $\operatorname{cov}(\hat{\mathbf{x}})=\mathbf{P}^{\mathrm{N}}=\left(\sum_{l=1}^{N} \mathbf{R}_{l}^{-1}\right)^{-1}$.

- Each $\mathbf{R}_{l}=\operatorname{cov}\left(\mathbf{y}_{l}\right)$ is an upper bound of $\mathbf{P}$. Be aware of that the difference $\mathbf{R}_{\imath}-\mathbf{R}_{\jmath}, \imath \neq \jmath$ might be indefinite, i.e., $\mathbf{R}_{\imath}$ and $\mathbf{R}_{\jmath}$ are incomparable and hence cannot be ordered.

- If $N=2$ and $\mathbf{R}_{1}=\mathbf{R}_{2}=\mathbf{R}_{12}$ then $\mathbf{P}=\mathbf{R}_{1}=\mathbf{R}_{2}$ is the optimal covariance of the estimate $\hat{\mathbf{x}}$.

\subsection{Estimation Under Unknown Cross-Correlations}

The result of naïvely neglecting the cross-correlations by assuming they are zero gives an estimate with an underestimated covariance. A concrete example of an underestimated covariance, which is the result of incorrectly assuming the crosscorrelations are zero, is provided in Example 3.1.

Example 3.1: Neglecting Cross-Correlations

Let $\mathbf{H}=\left[\begin{array}{ll}\mathbf{I} & \mathbf{I}\end{array}\right]^{\top}$ and $\mathbf{y}=\left[\begin{array}{ll}\mathbf{y}_{1}^{\top} & \mathbf{y}_{2}^{\top}\end{array}\right]^{\top}$ where the covariance structure of $\mathbf{y}_{1}$ and $\mathbf{y}_{2}$ are given by $\mathbf{R}=\left[\begin{array}{cc}\mathbf{R}_{1} & \mathbf{R}_{12} \\ \mathbf{R}_{12}^{1} & \mathbf{R}_{2}\end{array}\right]$ with

$$
\mathbf{R}_{1}=\left[\begin{array}{ll}
4 & 1 \\
1 & 2
\end{array}\right], \quad \mathbf{R}_{2}=\left[\begin{array}{cc}
2 & -1 \\
-1 & 4
\end{array}\right], \quad \mathbf{R}_{12}=\left[\begin{array}{ll}
2 & 0 \\
0 & 2
\end{array}\right] .
$$




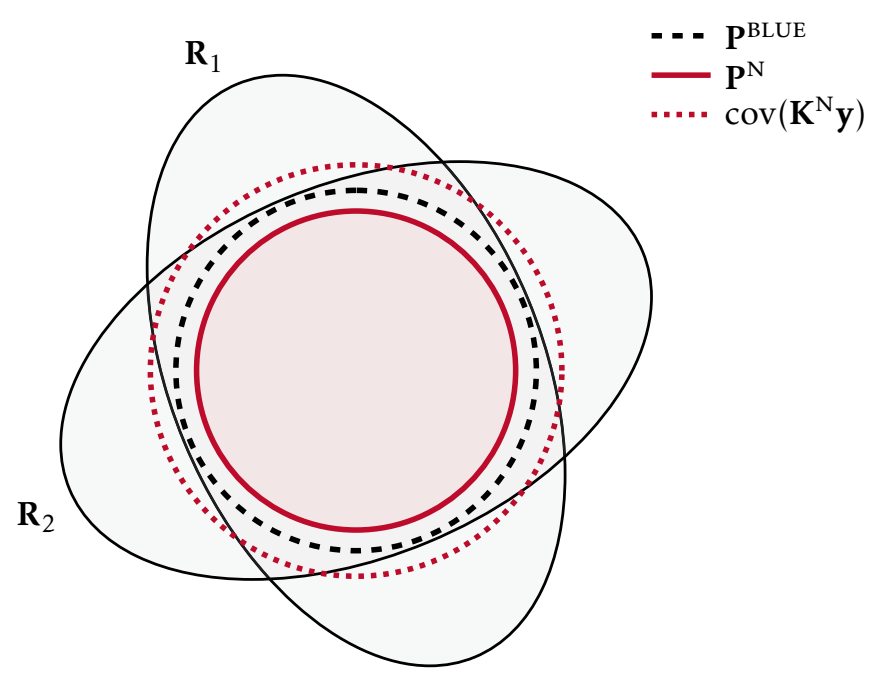

Figure 3.2: Illustration of the effect of neglecting cross-correlations. $\mathrm{P}^{\mathrm{N}}$ is the calculated covariance of the naïve estimate, $\operatorname{cov}\left(\mathrm{K}^{\mathrm{N}} \mathbf{y}\right)$ is the true covariance of the naïve estimate and $\mathbf{P}^{\mathrm{BLUE}}$ is the covariance of an optimal estimate using the true covariance $\mathbf{R}=\operatorname{cov}(\mathbf{y})$.

The gain and covariance of the BLUE are given by (2.24), i.e.,

$$
\begin{aligned}
& \mathbf{K}^{\text {BLUE }}=\left(\mathbf{H}^{\top} \mathbf{R}^{-1} \mathbf{H}\right)^{-1} \mathbf{H}^{\top} \mathbf{R}^{-1}, \\
& \mathbf{P}^{\text {BLUE }}=\left(\mathbf{H}^{\top} \mathbf{R}^{-1} \mathbf{H}\right)^{-1}=\frac{3}{2}\left[\begin{array}{ll}
1 & 0 \\
0 & 1
\end{array}\right] .
\end{aligned}
$$

Meanwhile, the gain and covariance of the naïve estimator of (2.57) is given by

$$
\begin{aligned}
& \mathbf{K}^{\mathrm{N}}=\left(\mathbf{R}_{1}^{-1}+\mathbf{R}_{2}^{-1}\right)^{-1}\left[\begin{array}{ll}
\mathbf{R}_{1}^{-1} & \mathbf{R}_{2}^{-1}
\end{array}\right]=\mathbf{P}^{\mathrm{N}}\left[\begin{array}{ll}
\mathbf{R}_{1}^{-1} & \mathbf{R}_{2}^{-1}
\end{array}\right], \\
& \mathbf{P}^{\mathrm{N}}=\left(\mathbf{R}_{1}^{-1}+\mathbf{R}_{2}^{-1}\right)^{-1}=\frac{7}{6}\left[\begin{array}{cc}
1 & 0 \\
0 & 1
\end{array}\right]<\mathbf{P}^{\text {BLUE }}
\end{aligned}
$$

As different gains are implied by the BLUE and the naive estimator, the corresponding estimates $\hat{\mathbf{x}}^{\mathrm{BLUE}}$ and $\hat{\mathbf{x}}^{\mathrm{N}}$ will differ. It is known from Theorem 2.1 that $\mathbf{K}^{\mathrm{BLUE}}$ is the gain of the BLUE estimate and that for any other gain $\mathbf{K}^{\prime}$ it holds that

$$
\mathbf{K}^{\prime} \neq \mathbf{K}^{\mathrm{BLUE}} \Longrightarrow \operatorname{cov}\left(\mathbf{K}^{\prime} \mathbf{y}\right) \geq \operatorname{cov}\left(\mathbf{K}^{\mathrm{BLUE}} \mathbf{y}\right)=\mathbf{P}^{\mathrm{BLUE}},
$$

and in particular $\operatorname{cov}\left(\mathbf{K}^{\mathrm{N}} \mathbf{y}\right) \geq \mathbf{P}^{\mathrm{BLUE}}$ where

$$
\operatorname{cov}\left(\mathbf{K}^{\mathrm{N}} \mathbf{y}\right)=\mathbf{K}^{\mathrm{N}} \mathbf{R}\left(\mathbf{K}^{\mathrm{N}}\right)^{\top}=\frac{35}{18}\left[\begin{array}{ll}
1 & 0 \\
0 & 1
\end{array}\right] .
$$

The results are shown in Figure 3.2. As seen, neglecting the cross-correlations underestimates the covariance, i.e., $\operatorname{cov}\left(\mathbf{K}^{\mathrm{N}} \mathbf{y}\right)>\mathbf{P}^{\mathrm{N}}$. 
Example 3.1 illustrates two important aspects. First, neglecting the crosscorrelations yields an underestimated covariance of $\hat{\mathbf{x}}^{\mathrm{N}}=\mathbf{K}^{\mathrm{N}} \mathbf{y}$. Secondly, neglecting the cross-correlations yields a gain matrix $\mathbf{K}^{\mathrm{N}}$ that is not optimal and hence $\operatorname{cov}\left(\mathbf{K}^{\mathrm{N}} \mathbf{y}\right) \geq \mathbf{P}^{\mathrm{BLUE}}$ where $\mathbf{P}^{\mathrm{BLUE}}$ is the covariance of the optimal estimate. Neglecting the cross-correlation is thus a bad choice for two reasons. More specifically, in Example 3.1

$$
\operatorname{cov}\left(\mathbf{K}^{\mathrm{N}} \mathbf{y}\right)>\mathbf{P}^{\mathrm{BLUE}}>\mathbf{P}^{\mathrm{N}}
$$

while in general only

$$
\operatorname{cov}\left(\mathbf{K}^{\mathrm{N}} \mathbf{y}\right) \geq \mathbf{P}^{\mathrm{BLUE}},
$$

can be assured.

The problem of only knowing $\mathbf{R} \in \mathcal{K}$ is here approached conservatively by trying to calculate so called conservative bounds on $\operatorname{cov}(\hat{\mathbf{x}})$ and/or $\mathbf{R}$. The conservative strategy is a resolution to the underestimated covariance problem explained above.

An estimator reporting an estimate $\hat{\mathbf{x}}$ and covariance $\mathbf{P}$ fulfilling

$$
\mathbf{P} \geq \operatorname{cov}(\hat{\mathbf{x}})
$$

is a conservative estimator and the estimate itself is a conservative estimate.

In much of the literature on distributed and decentralized estimation the notion consistency is used. To avoid confusion with other definitions of consistency found within estimation theory the notions conservative estimator and conservative estimate will be used in this thesis. As described before, $\overline{\mathbf{P}}$ will be used for an covariance for which (3.18) is guaranteed, to distinguish it from any potential covariance $\mathbf{P}$ calculated for an estimate.

\subsection{Conservative Linear Unbiased Estimator}

The BLUE introduced in Section 2.2 has the useful properties of being linear and unbiased, and is the optimal estimator, in the MSE sense, among all such linear unbiased estimators. Knowing only that $\mathbf{R} \in \mathcal{R}$ complicates the estimator design and in practice means that the BLUE cannot be found. The proposed approach is to instead use a conservative linear unbiased estimator (CLUE). CLUE is defined in Definition 3.1. An example of a conservative estimate is provided in Example 3.2.

Definition 3.1 (Conservative Linear Unbiased Estimator). Given is $\mathbf{y}=\mathbf{H x}+\mathbf{v}$, where $\mathbf{x}$ is a true state and $\mathbf{v}$ is zero-mean random noise. An estimator on the linear form $\hat{\mathbf{x}}=\mathbf{K y}$, reporting an covariance $\mathbf{P}$ of $\hat{\mathbf{x}}$, is called a conservative linear unbiased estimator if $\mathrm{E} \hat{\mathbf{x}}=\mathbf{x}$ and

$$
\mathbf{P} \geq \operatorname{cov}(\hat{\mathbf{x}})
$$


Actually the CLUE has already been defined, implicitly in (3.12), since any unbiased estimator calculating $\hat{\mathbf{x}}=\mathbf{K y}$ and $\mathbf{P}$ for which

$$
\mathbf{P} \geq \mathbf{K} \mathbf{R}^{\prime} \mathbf{K}^{\top}, \forall \mathbf{R}^{\prime} \in \mathcal{R},
$$

holds will also satisfy $\mathbf{P} \geq \mathbf{K R K}^{\top}$ as $\mathbf{R} \in \mathfrak{R}$. Hence, this estimator is a CLUE.

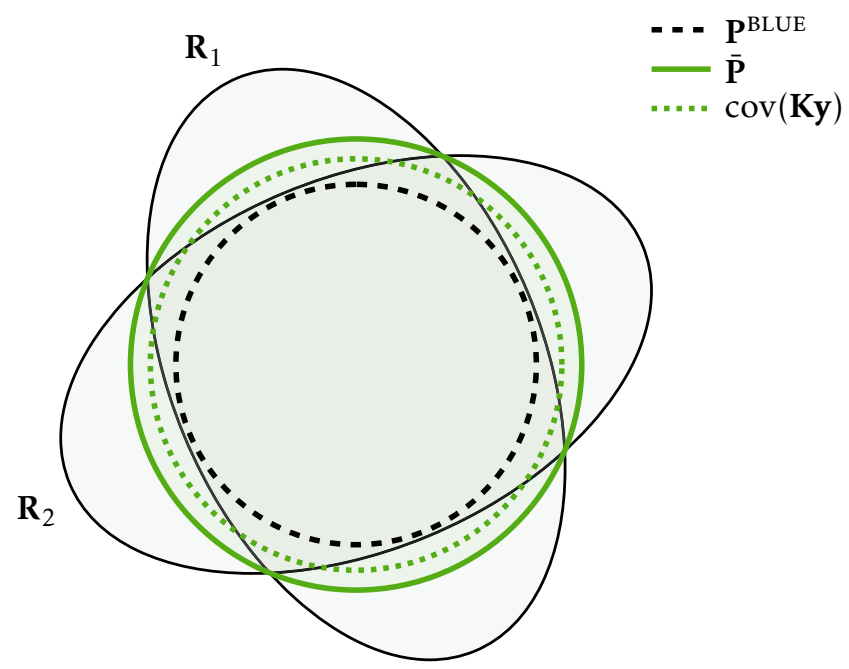

Figure 3.3: Example of a conservative estimator where $\overline{\mathbf{P}}$ is the covariance reported by a conservative estimator. $\mathbf{R}_{1}$ and $\mathbf{R}_{2}$ are both conservative bounds on the optimal covariance $\mathbf{P}^{\mathrm{BLUE}}$.

\section{Example 3.2: Conservative Estimate}

The same setup as in Example 3.1 is assumed, i.e., $\mathbf{H}=\left[\begin{array}{ll}\mathbf{I} & \mathbf{I}\end{array}\right]^{\top}$ and $\mathbf{y}=\left[\begin{array}{ll}\mathbf{y}_{1}^{\top} & \mathbf{y}_{2}^{\top}\end{array}\right]^{\top}$. The covariance is given by $\mathbf{R}=\left[\begin{array}{cc}\mathbf{R}_{1} & \mathbf{R}_{12} \\ \mathbf{R}_{12}^{\top} & \mathbf{R}_{2}\end{array}\right]$ where

$$
\mathbf{R}_{1}=\left[\begin{array}{ll}
4 & 1 \\
1 & 2
\end{array}\right], \quad \mathbf{R}_{2}=\left[\begin{array}{cc}
2 & -1 \\
-1 & 4
\end{array}\right], \quad \mathbf{R}_{12}=\left[\begin{array}{ll}
2 & 0 \\
0 & 2
\end{array}\right] .
$$

From Example 3.1 we have

$$
\begin{aligned}
\mathbf{K}^{\text {BLUE }} & =\left(\mathbf{H}^{\top} \mathbf{R}^{-1} \mathbf{H}\right)^{-1} \mathbf{H}^{\top} \mathbf{R}^{-1}, \\
\mathbf{P}^{\text {BLUE }} & =\left(\mathbf{H}^{\top} \mathbf{R}^{-1} \mathbf{H}\right)^{-1}=\frac{3}{2}\left[\begin{array}{ll}
1 & 0 \\
0 & 1
\end{array}\right] .
\end{aligned}
$$

Assume that another estimator reports the gain and covariance as

$$
\begin{aligned}
\mathbf{K} & =\left(\mathbf{R}_{1}^{-1}+\mathbf{R}_{2}^{-1}\right)^{-1}\left[\begin{array}{ll}
\mathbf{R}_{1}^{-1} & \mathbf{R}_{2}^{-1}
\end{array}\right], \\
\overline{\mathbf{P}} & =\frac{7}{3}\left[\begin{array}{ll}
1 & 0 \\
0 & 1
\end{array}\right],
\end{aligned}
$$


then $\hat{\mathbf{x}}=\mathbf{K y}$ is a conservative estimate since

$$
\overline{\mathbf{P}}-\operatorname{cov}(\mathbf{K y})=\overline{\mathbf{P}}-\mathbf{K R K}^{\top}=\frac{7}{3}\left[\begin{array}{ll}
1 & 0 \\
0 & 1
\end{array}\right]-\frac{35}{18}\left[\begin{array}{ll}
1 & 0 \\
0 & 1
\end{array}\right]>\mathbf{0},
$$

where $\mathbf{K R K}^{\top}=\frac{35}{18} \mathbf{I}$ has been calculated using $\mathbf{K}$ defined as above. The results are illustrated in Figure 3.3. The conservative estimator assumed in this case is the covariance intersection method studied in more detail in Chapter 4.

\subsection{Best Conservative Linear Unbiased Estimator}

While the CLUE provides a robust solution to the estimation problem where crosscorrelations are unknown, it is desirable to have a conservative analogue to the BLUE. That is, an estimator which is as little conservative as possible. This estimator will be called best conservative linear unbiased estimator (best CLUE).

Definition 3.2 (Best Conservative Linear Unbiased Estimator). An estimator reporting the estimate $\hat{\mathbf{x}}^{*}=\mathbf{K}^{*} \mathbf{y}$ and covariance $\mathbf{P}^{*}$ is called a best conservative linear unbiased estimator if $\mathbf{K}^{*}$ and $\mathbf{P}^{*}$ constitute the solution to

$$
\begin{array}{cl}
\mathbf{K}^{*}, \mathbf{P}^{*}=\underset{\mathbf{K}, \mathbf{P}}{\arg \min } & \mathbf{P} \\
\text { subject to } & \mathbf{K H}=\mathbf{I} \\
& \mathbf{P} \geq \mathbf{K R}^{\prime} \mathbf{K}^{\top}, \forall \mathbf{R}^{\prime} \in \mathfrak{R} \\
& \mathbf{P} \in \mathfrak{S}^{++} .
\end{array}
$$

Since also a simplified problem is studied herein, see Section 3.1.3, we will not only be dealing with a best CLUE, but also with a CLUE that is optimal w.r.t. the simplified problem. This CLUE will be called restricted best CLUE. It must be emphazised once again that finding a restricted best CLUE does not necessarily mean a best CLUE has been found since the two are solutions to different optimization problems.

A prerequisite for a restricted best CLUE is the minimum conservative bound (МСB), see Definition 3.3. In Definition 3.3 the notation $\mathbf{y}$ and $\mathbf{R}=\operatorname{cov}(\mathbf{y})$ is used for the random parameter and its covariance, respectively. The definition of a restricted best CLUE is provided in Definition 3.4. Note that also a best CLUE implicitly assumes an МСB can be found, see the minimization criterion of (3.12) or Definition 3.2.

Definition 3.3 (Minimum Conservative Bound). Let $\mathbf{R}=\operatorname{cov}(\mathbf{y})$ and assume it is only known that $\mathbf{R} \in \mathcal{R}$. Let $\overline{\mathfrak{R}}$ be a set containing all $\overline{\mathbf{R}}$ such that $\overline{\mathbf{R}} \geq \mathbf{R}^{\prime}, \forall \mathbf{R}^{\prime} \in$ $\mathfrak{R}$. A minimum conservative bound $\overline{\mathbf{R}}^{*} \in \overline{\mathcal{R}}$, if it exists, is given by

$$
\overline{\mathbf{R}} \geq \overline{\mathbf{R}}^{*}, \quad \forall \overline{\mathbf{R}} \in \overline{\mathrm{R}} .
$$




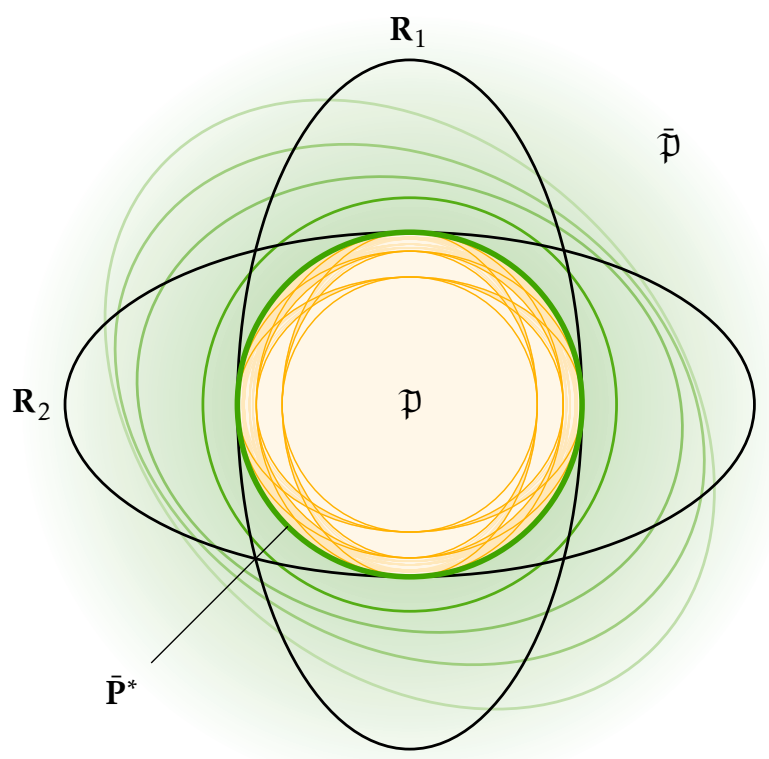

Figure 3.4: Illustration of the МСв $\overline{\mathbf{P}}^{*}$. The set $\mathfrak{P}$ contains all $\mathbf{P}^{\prime}=$ $\left(\mathbf{H}^{\top}\left(\mathbf{R}^{\prime}\right)^{-1} \mathbf{H}\right)^{-1}$ given the problem formulation. The set $\overline{\mathfrak{p}}$ contains all conservative bounds $\overline{\mathbf{P}}$, where also $\mathbf{R}_{1}, \mathbf{R}_{2} \in \overline{\mathfrak{p}}$. The yellow area resembles $\mathfrak{P}$ and the green area resembles $\overline{\mathfrak{p}}$.

Definition 3.4 (Restricted Best Conservative Linear Unbiased Estimator). An estimator reporting the estimate $\hat{\mathbf{x}}^{*}=\mathbf{K}^{*} \mathbf{y}$ and covariance $\mathbf{P}^{*}=\mathbf{K}^{*} \overline{\mathbf{R}}^{*}\left(\mathbf{K}^{*}\right)^{\top}$ is called a restricted best conservative linear unbiased estimator if $\mathbf{K}^{*}$ and $\overline{\mathbf{R}}^{*}$ constitute the solution to

$$
\begin{array}{ll}
\mathbf{K}^{*}, \overline{\mathbf{R}}^{*}=\underset{\mathbf{K}, \overline{\mathbf{R}}}{\arg \min } & \mathbf{P} \\
\text { subject to } & \mathbf{K H}=\mathbf{I} \\
& \mathbf{P}=\mathbf{K} \overline{\mathbf{R}} \mathbf{K}^{\top}, \forall \overline{\mathbf{R}} \in \overline{\mathbf{R}},
\end{array}
$$

where $\overline{\mathfrak{K}} \subseteq \mathfrak{S}^{++}$contains all block-diagonal $\overline{\mathbf{R}}$ for which $\overline{\mathbf{R}} \geq \mathbf{R}^{\prime}, \forall \mathbf{R}^{\prime} \in \mathcal{K}$ is satisfied, and $\mathcal{R}$ is the set to which $\mathbf{R}$ belong.

Next, a couple of examples of the MCB notion. Example 3.3 has been provided to concretize conservative bounds in general and the $\mathrm{MCB}$ concept in particular. Unfortunately an МСB does not always exist, as is demonstrated in Example 3.4. Example 3.4 is similar to Example 3.3 except for the conditions on the cross-covariance $\mathbf{R}_{12}$ which in that is assumed completely unknown. 


\section{Example 3.3: Minimum Conservative Bound}

Let $\overline{\mathfrak{P}}$ be the set of all $\overline{\mathbf{P}}$ that satisfies $\overline{\mathbf{P}} \geq \mathbf{P}^{\prime}, \forall \mathbf{P}^{\prime} \in \mathfrak{P}$, where the set $\mathfrak{P}$ spans all matrices generated as $\mathbf{P}^{\prime}=\left(\mathbf{H}^{\top}\left(\mathbf{R}^{\prime}\right)^{-1} \mathbf{H}\right)^{-1}$ when $\mathbf{R}^{\prime}$ is varied over all elements of $\mathfrak{R}$. The objective is to find an MСв $\overline{\mathbf{P}}^{*} \in \overline{\mathfrak{p}}$ which satisfies $\overline{\mathbf{P}} \geq \overline{\mathbf{P}}^{*}, \forall \overline{\mathbf{P}} \in \overline{\mathfrak{p}}$.

Assume $\mathbf{H}=\left[\begin{array}{ll}\mathbf{I} & \mathbf{I}\end{array}\right]^{\top}$. Let $\mathbf{y}=\left[\begin{array}{ll}\mathbf{y}_{1}^{\top} & \mathbf{y}_{2}^{\top}\end{array}\right]^{\top}$ and $\mathbf{R}=\operatorname{cov}(\mathbf{y})=\left[\begin{array}{ll}\mathbf{R}_{1} & \mathbf{R}_{12} \\ \mathbf{R}_{21} & \mathbf{R}_{2}\end{array}\right]$ be a block matrix consisting of four $2 \times 2$ blocks where it is known that $\mathbf{R}_{12}$ is diagonal but where the diagonal entries are unknown. This means $k$ is the set of all

$$
\mathbf{R}^{a, b}=\left[\begin{array}{cccc}
1 & 0 & a & 0 \\
0 & 4 & 0 & b \\
a & 0 & 4 & 0 \\
0 & b & 0 & 1
\end{array}\right]>\mathbf{0}
$$

that can be constructed by varying the unknowns $a$ and $b$ subject to $\mathbf{R}^{a, b}>\mathbf{0}$, which implies $a=\rho_{a} \sqrt{1 \cdot 4}=2 \rho_{a}$ and likewise $b=2 \rho_{b}$ where $\rho_{a}, \rho_{b} \in(-1,1)$. To find an MCB we proceed as follows. Applying the WLS formula of (2.54) yields

$$
\mathbf{P}^{a, b}=\left(\mathbf{H}^{\top}\left(\mathbf{R}^{a, b}\right)^{-1} \mathbf{H}\right)^{-1}=\left[\begin{array}{cc}
\frac{a^{2}-4}{2 a-5} & 0 \\
0 & \frac{b^{2}-4}{2 b-5}
\end{array}\right],
$$

which is an axis-aligned ellipse with eigenvalues $\lambda_{1}(a)=\frac{a^{2}-4}{2 a-5}$ and $\lambda_{2}(b)=\frac{b^{2}-4}{2 b-5}$.

In this case it is possible to find $\mathbf{P}_{\max }^{\prime} \in \mathfrak{P}$ such that $\mathbf{P}_{\max }^{\prime} \geq \mathbf{P}^{\prime}, \forall \mathbf{P}^{\prime} \in \mathfrak{P}$ by maximizing each diagonal entry of $\mathbf{P}^{a, b}$ separately. The eigenvalues are maximized when $a$ and $b$ are respectively given by

$$
a_{\max }=\underset{a}{\arg \max } \lambda_{1}(a)=\frac{5}{2} \pm \frac{3}{2}, \quad b_{\max }=\underset{b}{\arg \max } \lambda_{2}(b)=\frac{5}{2} \pm \frac{3}{2},
$$

where only $a_{\max }=1$ and $b_{\max }=1$ are valid. Plugging $a=a_{\max }$ and $b=b_{\max }$ into $\mathbf{P}^{a, b}$ yields

$$
\mathbf{P}_{\max }^{\prime}=\left[\begin{array}{ll}
1 & 0 \\
0 & 1
\end{array}\right],
$$

for which it holds $\mathbf{P}_{\max }^{\prime} \geq \mathbf{P}^{\prime}, \forall \mathbf{P}^{\prime} \in \mathfrak{P}$. Hence, in this case it will be sufficient to choose $\mathbf{P}_{\max }^{\prime}$ as a conservative bound and this bound will also be an MCB, i.e., $\overline{\mathbf{P}}^{*}=\mathbf{P}_{\text {max }}^{\prime}$ since $\overline{\mathbf{P}}^{*} \geq \mathbf{P}^{\prime}, \forall \mathbf{P}^{\prime} \in \mathfrak{P}$. No smaller $\overline{\mathbf{P}} \in \overline{\mathfrak{P}}$ can be found since this $\overline{\mathbf{P}} \in \overline{\mathfrak{P}}$ must satisfy $\overline{\mathbf{P}} \geq \mathbf{P}^{\prime}, \forall \mathbf{P}^{\prime} \in \mathfrak{P}$ and hence also $\overline{\mathbf{P}} \geq \mathbf{P}_{\text {max }}^{\prime}$, which cannot be true. The example is illustrated in Figure 3.4.

\section{Example 3.4: Minimum Conservative Bound Non-Existent}

Let $\overline{\mathfrak{P}}$ be the set of all $\overline{\mathbf{P}}$ that satisfies $\overline{\mathbf{P}} \geq \mathbf{P}^{\prime}, \forall \mathbf{P}^{\prime} \in \mathfrak{P}$, where the set $\mathfrak{P}$ spans all matrices generated as $\mathbf{P}^{\prime}=\left(\mathbf{H}^{\top}\left(\mathbf{R}^{\prime}\right)^{-1} \mathbf{H}\right)^{-1}$ when $\mathbf{R}^{\prime}$ is varied over all elements of $\mathfrak{R}$. The objective is to find an MCB $\overline{\mathbf{P}}^{*} \in \overline{\mathfrak{P}}$ which satisfies $\overline{\mathbf{P}} \geq \overline{\mathbf{P}}^{*}, \forall \overline{\mathbf{P}} \in \overline{\mathfrak{p}}$. Assume $\mathbf{H}=\left[\begin{array}{ll}\mathbf{I} & \mathbf{I}\end{array}\right]^{\top}$. Let $\mathbf{y}=\left[\begin{array}{ll}\mathbf{y}_{1}^{\top} & \mathbf{y}_{2}^{\top}\end{array}\right]^{\top}$ and $\mathbf{R}=\operatorname{cov}(\mathbf{y})=\left[\begin{array}{cc}\mathbf{R}_{1} & \mathbf{R}_{12} \\ \mathbf{R}_{21} & \mathbf{R}_{2}\end{array}\right]$ be a block matrix consisting of four $2 \times 2$ blocks where $\mathbf{R}_{12}$ is completely unknown. This 
means $\mathfrak{R}$ is given by all $\mathbf{R}^{a, b, c, d} \in \mathfrak{S}^{++}$parametrized as

$$
\mathbf{R}^{a, b, c, d}=\left[\begin{array}{llll}
1 & 0 & a & b \\
0 & 4 & c & d \\
a & c & 4 & 0 \\
b & d & 0 & 1
\end{array}\right]>\mathbf{0},
$$

where the entries of $\mathbf{R}_{12}=\left[\begin{array}{ll}a & b \\ c & d\end{array}\right]$ are unknown. The only constraint is

$$
\begin{aligned}
\mathbf{R}>\mathbf{0} & \Longleftrightarrow \mathbf{R}_{1}-\mathbf{R}_{12} \mathbf{R}_{2}^{-1} \mathbf{R}_{21}>\mathbf{0} \wedge \mathbf{R}_{1}>\mathbf{0} \\
& \Longleftrightarrow \mathbf{I}-\mathbf{R}_{1}^{-\frac{1}{2}} \mathbf{R}_{12} \mathbf{R}_{2}^{-\frac{1}{2}} \mathbf{R}_{2}^{-\frac{T}{2}} \mathbf{R}_{21} \mathbf{R}_{2}^{-\frac{T}{2}}>\mathbf{0} \wedge \mathbf{R}_{1}>\mathbf{0} \\
& \Longleftrightarrow \mathbf{I}-\mathbf{R}_{12}^{\prime} \mathbf{R}_{21}^{\prime}>\mathbf{0} \wedge \quad \mathbf{R}_{1}>\mathbf{0},
\end{aligned}
$$

where $\wedge$ represents logical and. Since $\mathbf{R}_{12}^{\prime} \mathbf{R}_{21}^{\prime}=\mathbf{R}_{12}^{\prime}\left(\mathbf{R}_{12}^{\prime}\right)^{\top} \geq \mathbf{0}$ the eigenvalues of $\mathbf{R}_{12}^{\prime} \mathbf{R}_{21}^{\prime}$ must be on the interval [0,1).

Applying the WLS formula of (2.54) yields

$$
\begin{aligned}
\mathbf{P}^{a, b, c, d}= & \left(\mathbf{H}^{\top}\left(\mathbf{R}^{a, b, c, d}\right)^{-1} \mathbf{H}\right)^{-1} \\
= & \frac{1}{b^{2}+2 b c+c^{2}+10 a+10 d-4 a d-25} \\
& \times\left[\begin{array}{cc}
8 a-2 a^{d}+5 a^{2}+c^{2}+4 b^{2}+2 a b c-20 & 4 a b-c-16 b+a c+4 b d+c d+b c^{2}+b^{c}-a b d-a c d \\
4 a b-c-16 b+a c+4 b d+c d+b c^{2}+b^{c}-a b d-a c d & 8 a-2 a d^{2}+5 d^{2}+c^{2}+4 b^{2}+2 b c d-20
\end{array}\right],
\end{aligned}
$$

which is an ellipse with orientation and eigenvalues depending on $a, b, c$ and $d$. As $\mathbf{H}=\left[\begin{array}{ll}\mathbf{I} & \mathbf{I}\end{array}\right]^{\top}$ and

$$
\min \left(\left[\mathbf{R}_{1}\right]_{i i},\left[\mathbf{R}_{2}\right]_{i i}\right)=1,
$$

where $i=1,2$, it should follow that $[\operatorname{cov}(\hat{\mathbf{x}})]_{i i} \leq 1$ since otherwise the information along some of the axes would have been decreased, compared to individually looking at each component of both $\mathbf{y}_{1}$ and $\mathbf{y}_{2}$. Hence, the concentric ellipses of all $\mathbf{P}^{a, b, c, d}$ should at least lie in a square with sides of length 2 .

In Figure 3.5 multiple $\mathbf{P}^{\prime} \in \mathfrak{P}$ are illustrated. The ellipses of $\mathbf{P}^{\prime} \in \mathfrak{P}$ are not only enclosed by the dashed square with sides of length 2 , but are also all contained in the intersection of the ellipses of $\mathbf{R}_{1}$ and $\mathbf{R}_{2}$. The intersection of $\mathbf{R}_{1}$ and $\mathbf{R}_{2}$ is itself contained in the dashed square.

In this case it is impossible to find an МСв. This can be understood using a geometrical argument, e.g., consider the ellipses of $\mathbf{R}_{1}, \mathbf{R}_{2}$ and $\overline{\mathbf{P}}$ in Figure 3.5. Each of these ellipses will only partly enclose the remaining two, i.e., neither $\mathbf{R}_{1} \geq \mathbf{R}_{2}, \overline{\mathbf{P}}$ nor $\mathbf{R}_{2} \geq \mathbf{R}_{1}, \overline{\mathbf{P}}$ nor $\overline{\mathbf{P}} \geq \mathbf{R}_{1}, \mathbf{R}_{2}$ hold. The same goes for all $\overline{\mathbf{P}}^{\prime} \in \overline{\mathfrak{p}}$. Hence, no MCB exists in this case.

In Example 3.3 a matrix $\mathbf{P}_{\max }^{\prime} \in \mathfrak{P}$ satisfying

$$
\mathbf{P}_{\max }^{\prime} \geq \mathbf{P}^{\prime}, \quad \forall \mathbf{P}^{\prime} \in \mathfrak{P},
$$




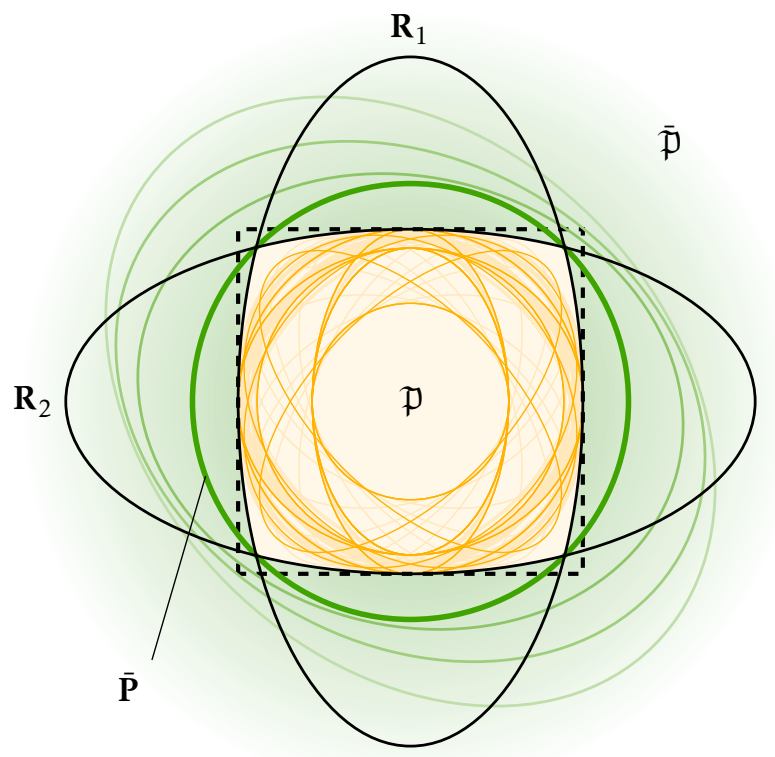

Figure 3.5: A scenario where no MCB exists, e.g., neither $\mathbf{R}_{1} \geq \mathbf{R}_{2}, \overline{\mathbf{P}}$ nor $\mathbf{R}_{2} \geq \mathbf{R}_{1}, \overline{\mathbf{P}}$ nor $\overline{\mathbf{P}} \geq \mathbf{R}_{1}, \mathbf{R}_{2}$. The yellow area resembles $\mathfrak{P}$ and the green area resembles $\overline{\mathfrak{p}}$.

could be found. Hence an MCB existed in that case given by $\overline{\mathbf{P}}^{*}=\mathbf{P}_{\max }^{\prime}$. On the other hand, no MCB could be found in Example 3.4. In Example 3.3

$$
\overline{\mathfrak{p}} \cap \mathfrak{P}=\overline{\mathbf{P}}^{*}=\mathbf{P}_{\text {max }}^{\prime},
$$

while in Example 3.4

$$
\overline{\mathfrak{P}} \cap \mathfrak{P}=\emptyset .
$$

Note, the relationship in (3.24) will not always be true just because an MCB exists.

The difference between Example 3.3 and Example 3.4 from an $\mathrm{MCB}$ perspective is the set $\mathfrak{p}$. In the former case the union

$$
\bigcup_{\mathbf{P}^{\prime} \in \mathfrak{P}} \mathcal{E}\left(\mathbf{0}, \mathbf{P}^{\prime}\right),
$$

is itself an ellipse. In the latter case the union in (3.26) is not an ellipse and an МСB does not exist in this case. These insights have been put together in an existence and uniqueness theorem, see Theorem 3.1. A scenario where an MCB $\overline{\mathbf{R}}^{*}$ cannot be found is provided by Example 3.5.

Theorem 3.1 (Existence and Uniqueness of Minimum Conservative Bounds). Let $\mathbf{R}=\operatorname{cov}(\mathbf{y})$ and assume it is only known that $\mathbf{R} \in \mathbb{R}$, where $\mathfrak{R} \subseteq \mathfrak{S}^{++}$. A minimum conservative bound exists if the union

$$
\bigcup_{\mathbf{R}^{\prime} \in \mathcal{R}} \mathcal{E}\left(\mathbf{0}, \mathbf{R}^{\prime}\right)
$$


is an ellipsoid. If a minimum conservative bound exists it is unique.

Proof: Let $\mathcal{U}=\cup_{\mathbf{R}^{\prime} \in \mathfrak{K}} \mathcal{E}\left(\mathbf{0}, \mathbf{R}^{\prime}\right)$. The set $\overline{\mathfrak{R}} \subset \mathfrak{S}^{++}$is a set spanning all $\overline{\mathbf{R}}$ for which $\overline{\mathbf{R}} \geq \mathbf{R}^{\prime}, \forall \mathbf{R}^{\prime} \in \mathcal{K}$ is fulfilled, or equivalently for which $\mathcal{U} \subseteq \mathcal{E}(\mathbf{0}, \overline{\mathbf{R}})$ is fulfilled.

If $\mathcal{U}$ is an ellipsoid it can be written on the form $\mathcal{E}(\mathbf{0}, \mathbf{S})$, for some $\mathbf{S} \in \mathfrak{S}^{++}$, such that

$$
\mathcal{U}=\mathcal{E}(\mathbf{0}, \mathbf{S}) .
$$

Since it is not possible to fit any other ellipsoid between $\mathcal{U}$ and $\mathcal{E}(\mathbf{0}, \mathbf{S})$ the matrix $\mathbf{S}$ satisfies

$$
\overline{\mathbf{R}} \geq \mathbf{S}, \quad \forall \overline{\mathbf{R}} \in \overline{\mathcal{R}} .
$$

Hence, a minimum conservative bound $\overline{\mathbf{R}}^{*}=\mathbf{S}$ exists.

If $\overline{\mathbf{R}}_{1}^{*} \in \overline{\mathcal{R}}$ and $\overline{\mathbf{R}}_{2}^{*} \in \overline{\mathcal{R}}$ are two minimum conservative bounds then by definition

$$
\overline{\mathbf{R}} \geq \overline{\mathbf{R}}_{1}^{*} \wedge \overline{\mathbf{R}} \geq \overline{\mathbf{R}}_{2}^{*}, \quad \forall \overline{\mathbf{R}} \in \overline{\mathcal{R}},
$$

implying that $\overline{\mathbf{R}}_{1}^{*} \geq \overline{\mathbf{R}}_{2}^{*}$ and $\overline{\mathbf{R}}_{2}^{*} \geq \overline{\mathbf{R}}_{1}^{*}$ must simultaneously be satisfied. These conditions can only be true at the same time if $\overline{\mathbf{R}}_{1}^{*}=\overline{\mathbf{R}}_{2}^{*}$. Hence, if a minimum conservative bound exists it is unique.

\section{Example 3.5: Conservative Bounds}

Let the off-diagonal elements of the $2 \times 2$ covariance matrix $\mathbf{R} \in \mathcal{R}$ be unknown. The set $\mathfrak{k}$ is generated by all matrices

$$
\mathbf{R}^{b}=\left[\begin{array}{ll}
4 & b \\
b & 1
\end{array}\right]>\mathbf{0}
$$

For $\mathbf{R}^{b}>\mathbf{0}$ to hold $b$ must satisfy $b=\rho \sqrt{4 \cdot 1}=2 \rho$, where $\rho \in(-1,1)$. Hence, a conservative bound $\overline{\mathbf{R}}$ in this case must simultaneously satisfy $\overline{\mathbf{R}} \geq \mathbf{R}^{b}, \forall b \in$ $(-2,2)$, i.e.,

$$
\overline{\mathbf{R}}-\mathbf{R}^{b}=\overline{\mathbf{R}}-\left[\begin{array}{cc}
4 & 2 \rho \\
2 \rho & 1
\end{array}\right] \geq \mathbf{0}, \quad \forall \rho \in(-1,1) .
$$

A number of conservative bounds are shown in Figure 3.6. All $\mathbf{R}^{\prime} \in \mathfrak{K}$ are contained in the outlined rectangle. No $\mathrm{MCB}$ can be found in this case since by definition the MCB requires a PSD condition to be satisfied, but the smallest conservative bounds considered here are given by incomparable matrices.

\subsubsection{Properties and Insights}

The existence of an MCB is a prerequisite for a (restricted) best CLUE to exist. If an MCB exists it is unique, see Theorem 3.1, but this does not necessarily mean that a (restricted) best CLUE is unique since multiple (restricted) best CLUEs might provide different estimates despite providing the same unique MCB.

In summary, a best CLUE has the following properties: 


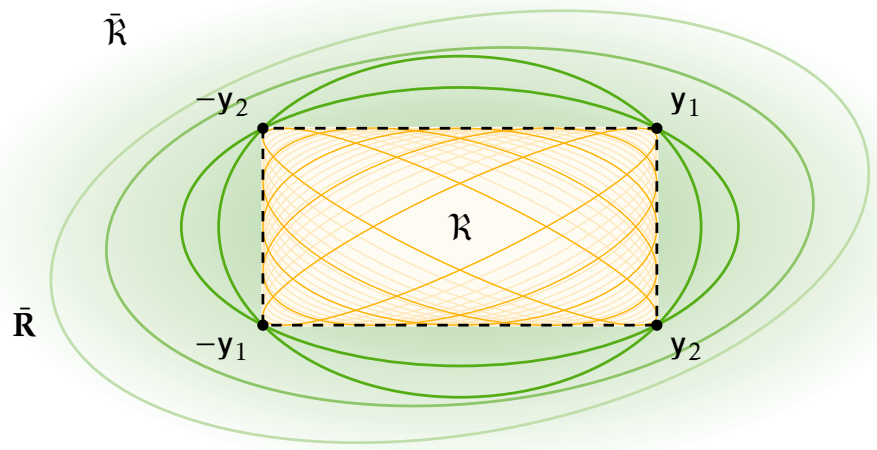

Figure 3.6: Illustration of several conservative bounds $\overline{\mathbf{R}}$ on $\mathbf{R}$. The ellipses of all $\mathbf{R}^{\prime} \in \mathcal{K}$ are contained in the dashed rectangle. The points $\mathbf{y}_{1}, \mathbf{y}_{2},-\mathbf{y}_{1}$ and $-y_{2}$ mark the four corners of the rectangle.

1. Linear: The estimate is calculated linearly as $\hat{\mathbf{x}}=\mathbf{K y}$.

2. Unbiased: The estimator is unbiased, i.e., $\mathrm{E} \hat{\mathbf{x}}=\mathbf{x}$.

3. Conservative: The estimator is conservative, i.e., $\mathbf{P} \geq \operatorname{cov}(\hat{\mathbf{x}})$, where $\mathbf{P}$ is the covariance reported by the estimator.

4. Optimal: The estimator provides the smallest covariance $\mathbf{P}=\overline{\mathbf{P}}^{*}$, in the positive definite sense, among all conservative linear unbiased estimators.

The difference between best CLUE and restricted best CLUE lies in how $\overline{\mathbf{P}}^{*}$ is calculated. In the latter case it is restricted according to Section 3.1.3. The restricted best CLUE is illustrated in Figure 3.7.

\subsubsection{Relaxed Conservative Bounds}

Theorem 3.1 provides the important insight that for general estimation problems an МСв does not exist. Nevertheless, the (restricted) best CLUE is not useless-it constitutes a solid theoretical concept to relate to in conservative estimation problems. To make the (restricted) best CLUE concept more useful the PSD conditions need to be relaxed. Below follows two relaxed variants:

1. Matrix loss function: Reformulate the MCB to replace $\overline{\mathbf{R}} \geq \overline{\mathbf{R}}^{*}$ with $J(\overline{\mathbf{R}}) \geq$ $J\left(\overline{\mathbf{R}}^{*}\right)$, where $J(\cdot)$ is a matrix loss function, e.g., the trace, the determinant or a matrix norm. Since trace is related to MSE it is a natural loss function for these problems. Matrix loss function relaxation is common in conservative estimation, see more on this in Chapter 4 .

2. Parametrization: Let $\overline{\mathbf{R}}(\theta)$ be a family of conservative bounds parametrized by $\theta$. Each family is characterized by a unique parametrization $\theta$. An $\mathrm{MCB}$ is then given as follows: Let a parametrized conservative bound be 


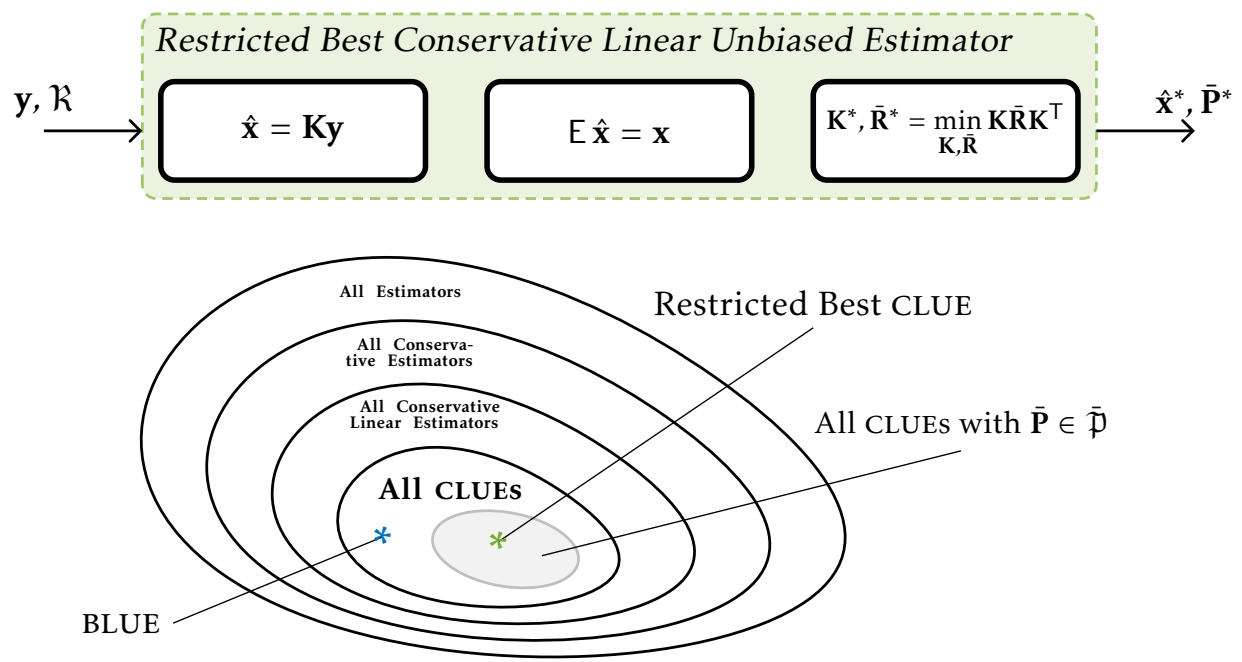

Figure 3.7: Illustration of the restricted best CLUE concept. A restricted best CLUE has to deal with the problem of only knowing that $\mathbf{R} \in \mathfrak{R}$.

given by $\overline{\mathbf{R}}^{*}\left(\theta^{*}\right)$ and another be given by $\overline{\mathbf{R}}(\theta)$. Denote a specific value of a parametrization $\theta$ by $\theta_{0}$. Then $\overline{\mathbf{R}}^{*}\left(\theta^{*}\right)$ is an MCB if for each $\theta_{0}$ there exists at least one $\theta_{0}^{*}$ such that

$$
\overline{\mathbf{R}}\left(\theta_{0}\right) \geq \overline{\mathbf{R}}^{*}\left(\theta_{0}^{*}\right) .
$$

The parametrized relaxation is formalized below.

As previously concluded, for general problems an МСB does not exists since we generally end up with the operation of comparing incomparable matrices, i.e., matrices $\overline{\mathbf{R}}$ and $\overline{\mathbf{R}}^{\prime}$ for which neither $\overline{\mathbf{R}} \geq \overline{\mathbf{R}}^{\prime}$ nor $\overline{\mathbf{R}}^{\prime} \geq \overline{\mathbf{R}}$ is true. See Section 2.1.2 for a small note on incomparable matrices. A workaround to this problem is to divide conservative bounds into different families according to which parametrization that can be used to describe them.

Each family $\mathbf{R}(\theta)$ is characterized by the parametrization given by $\theta$. What we want is to be able to compare two different parametrizations, say $\overline{\mathbf{R}}(\theta)$ and $\overline{\mathbf{R}}^{\prime}\left(\theta^{\prime}\right)$, by showing that for each $\theta_{0}^{\prime}$ there exists at least one $\theta_{0}$ such that

$$
\overline{\mathbf{R}}^{\prime}\left(\theta_{0}^{\prime}\right) \geq \overline{\mathbf{R}}\left(\theta_{0}\right)
$$

If this holds for the complete range of $\theta^{\prime}$ it is then possible to say

$$
\overline{\mathbf{R}}^{\prime}\left(\theta^{\prime}\right) \geq \overline{\mathbf{R}}(\theta),
$$

i.e., $\overline{\mathbf{R}}(\theta)$ is never larger than $\overline{\mathbf{R}}^{\prime}\left(\theta^{\prime}\right)$ in the parametric sense. For each $\theta^{\prime}=\theta_{0}^{\prime}$ the value $\theta=\theta_{0}$ is allowed to differ.

To get an intuition for this concept, consider the case in Figure 3.6 where neither of the conservative bounds that tightly encloses the dashed rectangle are 
smaller then the other. In that case the two innermost conservative bounds are given by

$$
\overline{\mathbf{R}}_{1}=\left[\begin{array}{ll}
8 & 0 \\
0 & 2
\end{array}\right], \quad \quad \overline{\mathbf{R}}_{2}=\left[\begin{array}{ll}
6 & 0 \\
0 & 3
\end{array}\right],
$$

which can also be written as

$$
\begin{aligned}
& \overline{\mathbf{R}}_{1}=\left[\begin{array}{ll}
8 & 0 \\
0 & 2
\end{array}\right]=\left[\begin{array}{cc}
\frac{4}{1 / 2} & 0 \\
0 & \frac{1}{1 / 2}
\end{array}\right]=\left[\begin{array}{cc}
\frac{4}{1 / 2} & 0 \\
0 & \frac{1}{1-1 / 2}
\end{array}\right]=\overline{\mathbf{R}}\left(\frac{1}{2}\right), \\
& \overline{\mathbf{R}}_{2}=\left[\begin{array}{ll}
6 & 0 \\
0 & 3
\end{array}\right]=\left[\begin{array}{cc}
\frac{4}{2 / 3} & 0 \\
0 & \frac{1}{1 / 3}
\end{array}\right]=\left[\begin{array}{cc}
\frac{4}{2 / 3} & 0 \\
0 & \frac{1}{1-2 / 3}
\end{array}\right]=\overline{\mathbf{R}}\left(\frac{2}{3}\right),
\end{aligned}
$$

where

$$
\overline{\mathbf{R}}(\theta)=\left[\begin{array}{cc}
\frac{4}{\theta} & 0 \\
0 & \frac{1}{1-\theta}
\end{array}\right] .
$$

The parametrization $\theta$ in this case defines both the structure in (3.32) and the range $\theta \in(0,1)$ of valid values of the parameter $\theta$.

Next it is shown that $\overline{\mathbf{R}}(\theta)$ intersects each of the points $\mathbf{y}_{1},-\mathbf{y}_{1}, \mathbf{y}_{2}$ and $-\mathbf{y}_{2}$, see Figure 3.8. The points $\mathrm{y}_{1}$ and $\mathrm{y}_{2}$ defining the dashed rectangle in Figure 3.8 are given by

$$
\mathrm{y}_{1}=\left[\begin{array}{l}
\sqrt{4} \\
\sqrt{1}
\end{array}\right]=\left[\begin{array}{l}
2 \\
1
\end{array}\right], \quad \mathrm{y}_{2}=\left[\begin{array}{c}
\sqrt{4} \\
-\sqrt{1}
\end{array}\right]=\left[\begin{array}{c}
2 \\
-1
\end{array}\right]
$$

Plugging the first component of $\mathbf{y}_{1}$ and $\mathbf{y}_{2}$ into the quadratic form that defines the ellipse $\overline{\mathbf{R}}(\theta)$ yields

$$
\begin{aligned}
& {\left[\begin{array}{l}
2 \\
y
\end{array}\right]^{\top}(\overline{\mathbf{R}}(\theta))^{-1}\left[\begin{array}{l}
2 \\
y
\end{array}\right]=1 } \\
\Longleftrightarrow & \theta \frac{4}{4}+(1-\theta) \frac{y^{2}}{1}=1 \\
\Longleftrightarrow & (1-\theta) y^{2}=1-\theta \\
\Longrightarrow & y= \pm 1,
\end{aligned}
$$

where \pm 1 are the second components of the corresponding points. From symmetry it follows that $\overline{\mathbf{R}}(\theta)$ also passes through $-\mathbf{y}_{1}$ and $-\mathbf{y}_{2}$. Hence, the parametrized conservative bound $\overline{\mathbf{R}}(\theta)>\mathbf{0}$ intersects $\mathbf{y}_{1},-\mathbf{y}_{1}, \mathbf{y}_{2}$ and $-\mathbf{y}_{2}$ for all $\theta \in(0,1)$, and thus parametrizes all ellipses that tightly encloses the dashed rectangle of Figure 3.8. Tightly here refers to the ellipses that intersect all four corners of the rectangle in Figure 3.8.

Figure 3.8 also illustrates one realization of

$$
\overline{\mathbf{R}}^{\prime}\left(\theta^{\prime}\right)=\left[\begin{array}{ll}
a & b \\
b & d
\end{array}\right], \quad \text { subject to } \quad \mathbf{y}_{2}^{\top}\left(\overline{\mathbf{R}}^{\prime}\left(\theta^{\prime}\right)\right)^{-1} \mathbf{y}_{2}=1,
$$




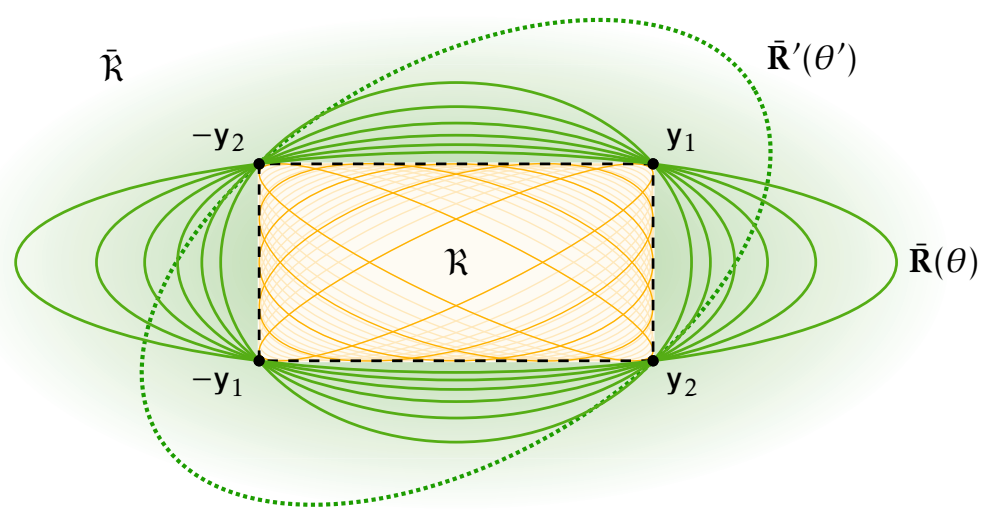

Figure 3.8: Illustration of parametized conservative bounds. Several ellipses of the minimum parametrized conservative bound $\overline{\mathbf{R}}(\theta)$ are provided.

which parametrizes all ellipses that passes through $y_{2}$ and $-y_{2}$. Since the ellipses generated by $\overline{\mathbf{R}}^{\prime}\left(\theta^{\prime}\right)$ does not generally provide a tight bound of the rectangle it will always be possible to find one value of $\theta$ such that $\overline{\mathbf{R}}^{\prime}\left(\theta^{\prime}\right) \geq \overline{\mathbf{R}}(\theta)$. This concept of tightness and the implications thereof are derived in [24, 25]. Hence we have

$$
\overline{\mathbf{R}}^{\prime}\left(\theta^{\prime}\right) \geq \overline{\mathbf{R}}(\theta),
$$

in the parametric sense.

\subsection{Conservative Linear Least Squares Estimation}

The WLS estimator is introduced in Section 2.3. In the Gauss-Markov Theorem it is shown that the WLS is also the BLUE when the linear model in (2.1) applies and $\mathbf{W}=\mathbf{R}^{-1}$ is used as the weight matrix.

The WLS estimator can also be used in conservative linear estimation. The WLS estimate is given by

$$
\begin{aligned}
\hat{\mathbf{x}} & =\left(\mathbf{H}^{\top} \overline{\mathbf{R}}^{-1} \mathbf{H}\right)^{-1} \mathbf{H}^{\top} \overline{\mathbf{R}}^{-1} \mathbf{y}, \\
\overline{\mathbf{P}} & =\left(\mathbf{H}^{\top} \overline{\mathbf{R}}^{-1} \mathbf{H}\right)^{-1},
\end{aligned}
$$

where $\overline{\mathbf{R}}$ is a conservative bound of $\mathbf{R}$. The expressions in (3.35) are equivalent to the expressions of the WLS estimator in (2.54), but where $\mathbf{R}$ has been replaced by a conservative bound $\overline{\mathbf{R}}$. The WLS structure in (3.35) has been suggested in, e.g., $[9,10]$.

A conservative analogue of the Gauss-Markov theorem is provided below in Theorem 3.2. The theorem does not provide any revolutionary conclusions, but it tells us that the problem behaves as it should and can be used as a tool to derive new theoretical results, see Chapter 4. 
Theorem 3.2 (Conservative Gauss-Markov Theorem). Consider the data y given on the form

$$
\mathbf{y}=\mathbf{H x}+\mathbf{v}
$$

where $\mathbf{x}$ is the true state to be estimated, $\mathbf{v}$ is zero-mean noise, $\mathbf{R}=\operatorname{cov}(\mathbf{y})$ and $\mathbf{H}$ is full rank. Assume it is only known that $\mathbf{R} \in \mathfrak{R}$. Assume $\overline{\mathbf{R}}^{*} \in \overline{\mathcal{K}}$ is the minimum conservative bound of $\mathbf{R}$, i.e.,

$$
\overline{\mathbf{R}} \geq \overline{\mathbf{R}}^{*}, \quad \forall \overline{\mathbf{R}} \in \overline{\mathcal{R}},
$$

where $\overline{\mathcal{K}}$ contains all $\overline{\mathbf{R}}$ that fulfills $\overline{\mathbf{R}} \geq \mathbf{R}^{\prime}, \forall \mathbf{R}^{\prime} \in \mathcal{K}$. Then, among all linear unbiased estimators Ky reporting the error covariance $\mathbf{K} \overline{\mathbf{R}} \mathbf{K}^{\top}$, the restricted best conservative linear unbiased estimator is given by

$$
\hat{\mathbf{x}}^{*}=\left(\mathbf{H}^{\top}\left(\overline{\mathbf{R}}^{*}\right)^{-1} \mathbf{H}\right)^{-1} \mathbf{H}^{\top}\left(\overline{\mathbf{R}}^{*}\right)^{-1} \mathbf{y},
$$

with error covariance given by

$$
\overline{\mathbf{P}}^{*}=\left(\mathbf{H}^{\top}\left(\overline{\mathbf{R}}^{*}\right)^{-1} \mathbf{H}\right)^{-1} .
$$

Proof: The following will be shown:

1. For any $\overline{\mathbf{R}}$ it holds that $\mathbf{K} \overline{\mathbf{R}} \mathbf{K}^{\top} \geq\left(\mathbf{H}^{\top} \overline{\mathbf{R}}^{-1} \mathbf{H}\right)^{-1}$ for all $\mathbf{K}$ fulfilling $\mathbf{K H}=\mathbf{I}$

2. $\overline{\mathbf{R}} \geq \overline{\mathbf{R}}^{*} \Longrightarrow\left(\mathbf{H}^{\top} \overline{\mathbf{R}}^{-1} \mathbf{H}\right)^{-1} \geq\left(\mathbf{H}^{\top}\left(\overline{\mathbf{R}}^{*}\right)^{-1} \mathbf{H}\right)^{-1}$

3. $\left(\mathbf{H}^{\top}\left(\overline{\mathbf{R}}^{*}\right)^{-1} \mathbf{H}\right)^{-1} \geq \operatorname{cov}\left(\hat{\mathbf{x}}^{*}\right)$

1. An arbitrary linear estimator is constructed as $\hat{\mathbf{x}}=\mathbf{K y}$ with a conservative covariance $\overline{\mathbf{P}}=\mathbf{K} \overline{\mathbf{R}} \mathbf{K}^{\top}$. From the unbiasedness constraint we have $\mathbf{K H}=\mathbf{I}$. For the specific conservative bound $\overline{\mathbf{R}}$, we thus have the matrix difference

$$
\begin{aligned}
\overline{\mathbf{P}}-\left(\mathbf{H}^{\top} \overline{\mathbf{R}}^{-1} \mathbf{H}\right)^{-1} & =\mathbf{K} \overline{\mathbf{R}} \mathbf{K}^{\top}-\left(\mathbf{H}^{\top} \overline{\mathbf{R}}^{-1} \mathbf{H}\right)^{-1} \\
& =\mathbf{K} \overline{\mathbf{R}} \mathbf{K}^{\top}-\mathbf{K} \mathbf{H}\left(\mathbf{H}^{\top} \overline{\mathbf{R}}^{-1} \mathbf{H}\right)^{-1} \mathbf{H}^{\top} \mathbf{K}^{\top} \\
& =\mathbf{K}\left(\overline{\mathbf{R}}-\mathbf{H}\left(\mathbf{H}^{\top} \overline{\mathbf{R}}^{-1} \mathbf{H}\right)^{-1} \mathbf{H}^{\top}\right) \mathbf{K}^{\top} \\
& =\mathbf{K}\left(\mathbf{I}-\mathbf{H}\left(\mathbf{H}^{\top} \overline{\mathbf{R}}^{-1} \mathbf{H}\right)^{-1} \mathbf{H}^{\top} \overline{\mathbf{R}}^{-1}\right) \overline{\mathbf{R}} \mathbf{K}^{\top} \\
& =\mathbf{K}\left(\mathbf{I}-\mathcal{P}_{\mathbf{H}}\right) \overline{\mathbf{R}} \mathbf{K}^{\top},
\end{aligned}
$$

where $\mathbf{I}-\mathcal{P}_{\mathbf{H}}$ is a projection matrix weighted by $\overline{\mathbf{R}}^{-1}$. Now, since $\overline{\mathbf{R}}$ and $\left(\mathbf{I}-\mathcal{P}_{\mathbf{H}}\right)$ are $\mathrm{PSD}$, and

$$
\left(\left(\mathbf{I}-\mathcal{P}_{\mathbf{H}}\right) \overline{\mathbf{R}}\right)^{\top}=\left(\mathbf{I}-\mathcal{P}_{\mathbf{H}}\right) \overline{\mathbf{R}},
$$

the matrix product $\left(\mathbf{I}-\mathcal{P}_{\mathbf{H}}\right) \overline{\mathbf{R}}$ is PSD [19], and hence we arrive at

$$
\mathbf{K}\left(\mathbf{I}-\mathcal{P}_{\mathbf{H}}\right) \overline{\mathbf{R}} \mathbf{K}^{\top} \geq \mathbf{0},
$$

implying that $\mathbf{K} \overline{\mathbf{R}} \mathbf{K}^{\top} \geq\left(\mathbf{H}^{\top} \overline{\mathbf{R}}^{-1} \mathbf{H}\right)^{-1}$ for arbitrary $\mathbf{K}$ compliant with the unbiasedness constraint, and for all $\overline{\mathbf{R}}$. 
2. As $\overline{\mathbf{R}}$ and $\overline{\mathbf{R}}^{*}$ are PD we have from Section 2.1 .2 that

$$
\overline{\mathbf{R}} \geq \overline{\mathbf{R}}^{*} \Longleftrightarrow\left(\overline{\mathbf{R}}^{*}\right)^{-1} \geq \overline{\mathbf{R}}^{-1},
$$

and hence

$$
\begin{aligned}
\overline{\mathbf{R}} \geq \overline{\mathbf{R}}^{*} & \Longleftrightarrow\left(\overline{\mathbf{R}}^{*}\right)^{-1}-\overline{\mathbf{R}}^{-1} \geq \mathbf{0} \\
& \Longleftrightarrow \mathbf{H}^{\top}\left(\left(\overline{\mathbf{R}}^{*}\right)^{-1}-\overline{\mathbf{R}}^{-1}\right) \mathbf{H} \geq \mathbf{0} \\
& \Longleftrightarrow\left(\mathbf{H}^{\top} \overline{\mathbf{R}}^{-1} \mathbf{H}\right)^{-1}-\left(\mathbf{H}^{\top}\left(\overline{\mathbf{R}}^{*}\right)^{-1} \mathbf{H}\right)^{-1} \geq \mathbf{0} .
\end{aligned}
$$

3. Using $\hat{\mathbf{x}}^{*}=\left(\mathbf{H}^{\top}\left(\overline{\mathbf{R}}^{*}\right)^{-1} \mathbf{H}\right)^{-1} \mathbf{H}^{\top}\left(\overline{\mathbf{R}}^{*}\right)^{-1} \mathbf{y}$ and $\mathbf{v}=\mathbf{y}-\mathbf{H x}$ we get

$$
\begin{aligned}
\operatorname{cov}\left(\hat{\mathbf{x}}^{*}\right) & =\mathrm{E}\left(\left(\mathbf{H}^{\top}\left(\overline{\mathbf{R}}^{*}\right)^{-1} \mathbf{H}\right)^{-1} \mathbf{H}^{\top}\left(\overline{\mathbf{R}}^{*}\right)^{-1} \mathbf{y}-\left(\mathbf{H}^{\top}\left(\overline{\mathbf{R}}^{*}\right)^{-1} \mathbf{H}\right)^{-1} \mathbf{H}^{\top}\left(\overline{\mathbf{R}}^{*}\right)^{-1} \mathbf{H} \mathbf{x}\right)(\cdot)^{\top} \\
& =\left(\mathbf{H}^{\top}\left(\overline{\mathbf{R}}^{*}\right)^{-1} \mathbf{H}\right)^{-1} \mathbf{H}^{\top}\left(\overline{\mathbf{R}}^{*}\right)^{-1}\left(\mathrm{E} \mathbf{v v}^{\top}\right)\left(\overline{\mathbf{R}}^{*}\right)^{-1} \mathbf{H}\left(\mathbf{H}^{\top}\left(\overline{\mathbf{R}}^{*}\right)^{-1} \mathbf{H}\right)^{-1} \\
& =\left(\mathbf{H}^{\top}\left(\overline{\mathbf{R}}^{*}\right)^{-1} \mathbf{H}\right)^{-1} \mathbf{H}^{\top}\left(\overline{\mathbf{R}}^{*}\right)^{-1} \mathbf{R}\left(\overline{\mathbf{R}}^{*}\right)^{-1} \mathbf{H}\left(\mathbf{H}^{\top}\left(\overline{\mathbf{R}}^{*}\right)^{-1} \mathbf{H}\right)^{-1}
\end{aligned}
$$

where $\mathbf{R}=\mathbf{E} \mathbf{v v} \mathbf{v}^{\top}$ has been used. By assumption $\overline{\mathbf{R}}^{*} \geq \mathbf{R}$ such that $\operatorname{cov}\left(\hat{\mathbf{x}}^{*}\right)$ can be bounded as

$$
\begin{aligned}
\operatorname{cov}\left(\hat{\mathbf{x}}^{*}\right) & \leq\left(\mathbf{H}^{\top}\left(\overline{\mathbf{R}}^{*}\right)^{-1} \mathbf{H}\right)^{-1} \mathbf{H}^{\top}\left(\overline{\mathbf{R}}^{*}\right)^{-1} \overline{\mathbf{R}}^{*}\left(\overline{\mathbf{R}}^{*}\right)^{-1} \mathbf{H}\left(\mathbf{H}^{\top}\left(\overline{\mathbf{R}}^{*}\right)^{-1} \mathbf{H}\right)^{-1} \\
& =\left(\mathbf{H}^{\top}\left(\overline{\mathbf{R}}^{*}\right)^{-1} \mathbf{H}\right)^{-1} \\
& =\overline{\mathbf{P}}^{*}
\end{aligned}
$$

A consequence of Theorem 3.2 is that finding the МСв $\overline{\mathbf{P}}^{*}$ is equivalent to finding the MCB $\overline{\mathbf{R}}^{*}$, if the linear model $\mathbf{y}=\mathbf{H x}+\mathbf{v}$ is valid and only linear unbiased estimators $\mathbf{K y}$, reporting an covariance $\mathbf{K} \overline{\mathbf{R}} \mathbf{K}^{\top}$, are considered where $\overline{\mathbf{R}} \in \overline{\mathbf{R}}$. In other words, looking for $\overline{\mathbf{P}}^{*}$ can be replaced by a search for $\overline{\mathbf{R}}^{*}$ followed by an application of the WLS estimator-if $\overline{\mathbf{R}}^{*}$ is found then a restricted best CLUE can be found. Theorem 3.2 works equally well for the parametrized relaxation. In this case the МСв $\overline{\mathbf{R}}^{*}=\overline{\mathbf{R}}^{*}\left(\theta^{*}\right)$ is used instead.

Using $\mathbf{W}=\left(\overline{\mathbf{R}}^{*}\right)^{-1}$ instead of $\mathbf{W}=\mathbf{R}^{-1}$ makes a restricted best CLUE suboptimal compared to the BLUE, basically because the gain used by the BLUE cannot be calculated. However, this CLUE is optimal by the definition of the conservative estimation problem where it is only known that $\mathbf{R} \in \mathcal{R}$. If $\mathcal{R}=\{\mathbf{R}\}$ then $\overline{\mathbf{R}}^{*}=\mathbf{R}$ and the conservative estimation problem reduces to the classical linear estimation problem, i.e., a restricted best CLUE reduces to the BLUE. 



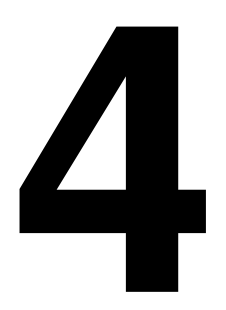

\section{Conservative Linear Unbiased Fusion}

CONSERVATIVE FUSION refers to fusion problems ${ }^{1}$ solved using conservative estimation techniques. In this chapter several conservative fusion methods are described together with their implicitly assumed cross-correlation structure. As will be seen, an important aspect in fusion of correlated estimates is the specific structure of the actual underlying cross-correlations between the estimates. It is the cross-correlation structure that determines how small the covariance of the optimal conservative estimator is allowed to be.

In Chapter 3 the notation $\mathbf{y}$ and $\mathbf{R} \in \mathcal{K}$ is used to describe the data which is input to the estimator. In this chapter we return to the notation

$$
\mathbf{y} \longrightarrow \hat{\mathbf{z}}_{J}, \quad \mathbf{R} \longrightarrow \mathbf{C}_{J}, \quad \mathbf{H} \longrightarrow \mathbf{H}_{J}, \quad \mathbf{K} \longrightarrow \mathbf{K}_{J},
$$

as we are specifically dealing with estimates and not general data $\mathbf{y}$. It is assumed $\operatorname{rank}\left(\mathbf{H}_{J}\right)=n_{x}$ such that $\mathbf{H}_{J}$ is full rank and the linear system of equations $\hat{\mathbf{z}}_{J}=$ $\mathbf{H}_{J} \mathbf{x}+\mathbf{e}_{J}$ is not underdetermined. The sets $\mathcal{k}$ and $\overline{\mathcal{k}}$ are replaced according to

$$
\mathfrak{K} \longrightarrow \mathbb{C}, \quad \overline{\mathfrak{K}} \longrightarrow \overline{\mathbb{C}} .
$$

Some parts of the joint covariance $\mathbf{C}_{J}$ are assumed unknown, but it is known that $\mathbf{C}_{J} \in \mathbb{C}$. The set $\overline{\mathbb{C}}$ spans all $\overline{\mathbf{C}}_{J}$ for which $\overline{\mathbf{C}}_{J} \geq \mathbf{C}_{J}^{\prime}, \forall \mathbf{C}_{J}^{\prime} \in \mathbb{C}$ is satisfied.

A case where $N=2$ will be studied to simplify the illustration of the crosscorrelations. Hence

$$
\hat{\mathbf{z}}_{J}=\left[\begin{array}{c}
\hat{\mathbf{z}}_{1} \\
\hat{\mathbf{z}}_{2}
\end{array}\right], \quad \mathbf{C}_{J}=\left[\begin{array}{cc}
\mathbf{C}_{1} & \mathbf{C}_{12} \\
\mathbf{C}_{21} & \mathbf{C}_{2}
\end{array}\right], \quad \mathbf{H}_{J}=\left[\begin{array}{l}
\mathbf{H}_{1} \\
\mathbf{H}_{2}
\end{array}\right], \quad \mathbf{K}_{J}=\left[\begin{array}{ll}
\mathbf{K}_{1} & \mathbf{K}_{2}
\end{array}\right] .
$$

It should however be emphasized that the described correlations can be generalized for arbitrary $N \geq 2$.

\footnotetext{
${ }^{1}$ As pointed out in Chapter 2, fusion problems refer to estimation problems where multiple estimates are combined to form an improved estimate.
} 
It is assumed that the input estimates $\hat{\mathbf{z}}_{\imath}$ are unbiased $\forall \imath$. Furthermore, it is assumed $\mathbf{C}_{l} \geq \operatorname{cov}\left(\hat{\mathbf{z}}_{\imath}\right)$ to allow for conservative estimates to be used, and not only estimates for which $\mathbf{C}_{\imath}=\operatorname{cov}\left(\hat{\mathbf{z}}_{\imath}\right)$. For cross-covariances we use $\mathbf{C}_{\imath \jmath}=\operatorname{cov}\left(\hat{\mathbf{z}}_{\imath}, \hat{\mathbf{z}}_{\jmath}\right)$. Hence

$$
\mathrm{C}_{J} \geq \operatorname{cov}\left(\hat{\mathbf{z}}_{J}\right)
$$

If, e.g., $\mathbb{C}$ is defined as

$$
\mathbb{C}=\left\{\mathbf{C}_{J}^{\prime}=\left[\begin{array}{cc}
\mathbf{C}_{1}^{\prime} & \mathbf{C}_{12}^{\prime} \\
\mathrm{C}_{21}^{\prime} & \mathbf{C}_{2}^{\prime}
\end{array}\right] \mid \mathbf{C}_{1}^{\prime} \leq \mathbf{C}_{1}, \mathbf{C}_{2}^{\prime} \leq \mathbf{C}_{2}, \mathbf{C}_{J}^{\prime} \geq \mathbf{0}\right\}
$$

then $\mathbf{C}_{J} \in \mathbb{C}$ implies $\operatorname{cov}\left(\hat{\mathbf{z}}_{J}\right) \in \mathbb{C}$ since $\mathbf{C}_{1} \geq \operatorname{cov}\left(\hat{\mathbf{z}}_{1}\right)$ and $\mathbf{C}_{2} \geq \operatorname{cov}\left(\hat{\mathbf{z}}_{2}\right)$ imply that for some $\mathbf{C}_{J}^{\prime} \in \mathbb{C}$ we have

$$
C_{J}^{\prime}=\left[\begin{array}{cc}
\operatorname{cov}\left(\hat{\mathbf{z}}_{1}\right) & \operatorname{cov}\left(\hat{\mathbf{z}}_{1}, \hat{\mathbf{z}}_{2}\right) \\
\operatorname{cov}\left(\hat{\mathbf{z}}_{2}, \hat{\mathbf{z}}_{1}\right) & \operatorname{cov}\left(\hat{\mathbf{z}}_{2}\right)
\end{array}\right] .
$$

For our purpose it does not matter if $\operatorname{cov}\left(\hat{\mathbf{z}}_{J}\right)$ is included in $\mathbb{C}$ if $\mathbf{C}_{J} \geq \operatorname{cov}\left(\hat{\mathbf{z}}_{J}\right)$, the important thing is that we have $\mathbf{C}_{J} \in \mathbb{C}$ since we only know $\mathbf{C}_{\imath} \geq \operatorname{cov}\left(\hat{\mathbf{z}}_{\imath}\right)$. We could therefore replace $(4.5)$ by

$$
\mathbb{C}=\left\{\mathbf{C}_{J}^{\prime}=\left[\begin{array}{cc}
\mathbf{C}_{1}^{\prime} & \mathbf{C}_{12}^{\prime} \\
\mathrm{C}_{21}^{\prime} & \mathrm{C}_{2}^{\prime}
\end{array}\right] \mid \mathbf{C}_{1}^{\prime}=\mathbf{C}_{1}, \mathbf{C}_{2}^{\prime}=\mathbf{C}_{2}, \mathbf{C}_{J}^{\prime} \geq \mathbf{0}\right\}
$$

For technical convenience we will however stick to (4.5).

\section{Considered Methods}

The different conservative fusion methods described here are distinguished by the correlation structure they assume. The following conservative fusion methods are considered:

- Covariance intersection [22], Section 4.1.2, makes no assumption about the underlying cross-correlation structure, i.e., the cross-correlations are regarded as completely unknown.

- Inverse covariance intersection [49], Section 4.2.2, assumes that the crosscorrelations can be decomposed into the correlated information structure, see Section 4.2.1.

- Largest ellipsoid method [5], Section 4.3.2, assumes the cross-correlations are component-wise aligned, see Section 4.3.1.

\section{Fusion Example}

Each method is illustrated by fusing the two-dimensional covariance matrices

$$
\mathrm{C}_{1}=\left[\begin{array}{ll}
4 & 0 \\
0 & 1
\end{array}\right], \quad \mathbf{C}_{2}=\left[\begin{array}{ll}
1 & 0 \\
0 & 4
\end{array}\right] .
$$




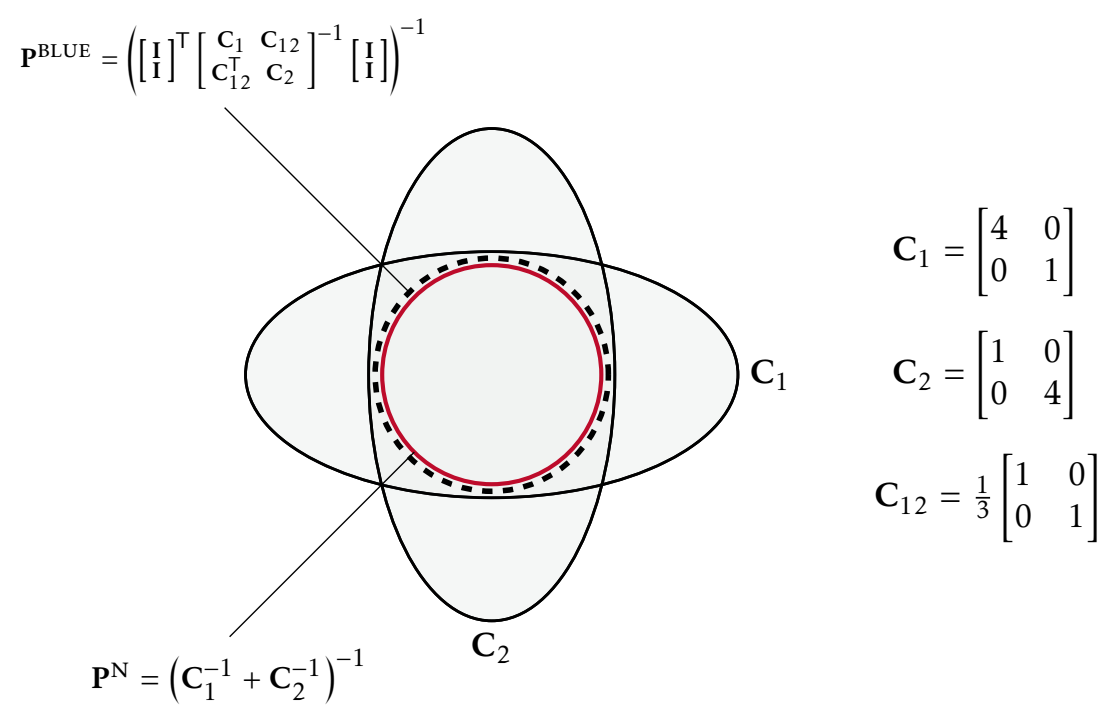

Figure 4.1: The fusion scenario used to illustrate the conservative fusion methods of this chapter. The covariance matrices of the estimates to be fused are given by $\mathbf{C}_{1}$ and $\mathbf{C}_{2}$. Also the covariance matrices $\mathbf{P}^{\mathrm{N}}$ and $\mathbf{P}^{\mathrm{BLUE}}$ of the naively fused estimate and the BLUE estimate have been provided.

The corresponding estimates $\hat{\mathbf{z}}_{1}$ and $\hat{\mathbf{z}}_{2}$, where $\mathbf{H}_{1}=\mathbf{H}_{2}=\mathbf{I}$, have been left out since in this case they are not of interest for illustration purpose. The crosscovariance $\mathbf{C}_{12}=\frac{1}{3} \mathbf{I}$ is assumed unknown for the conservative fusion methods.

A naïve estimator which assumes $C_{12}=\mathbf{0}$ and the BLUE with true joint covariance $\mathbf{C}_{12}=\frac{1}{3} \mathbf{I}$ are used for comparison. The fusion scenario is illustrated in Figure 4.1 together with the covariances $\mathbf{P}^{\mathrm{N}}$ and $\mathbf{P}^{\mathrm{BLUE}}$ of the naive estimate and the BLUE estimate, respectively.

\section{Contributions}

Similar to Chapter 3, several contributions are dispersed in this chapter. These contributions include:

Optimality aspects. In Theorem 4.2 and in Theorem $4.6 \mathrm{CI}$ and ICI are, respectively, shown being restricted best CLUEs under different assumptions about the cross-correlations. Theorem 4.8 proves LE is a best CLUE under yet another assumption about the cross-correlations.

Properties. For each method a number of properties, in addition to optimality aspects, are shown and discussed.

ICI for partial estimates. A modification of ICI to enable fusion of partial estimates is proposed in Algorithm 4.2. Conservativeness of this modification is proven in Theorem 4.5 . 
LE for partial estimates. An extension of the LE method is proposed in Algorithm 4.4 which can be used for fusion of one full estimate and one partial estimate.

\subsection{Fusion Under Completely Unknown Cross-Correlations}

The completely unknown cross-correlation structure is described below. Then CI is introduced and analyzed.

\subsubsection{Completely Unknown Cross-Correlation}

In some situations it is impossible to ensure any sort of structure in the crosscorrelations between the estimates to be fused. The only constraint on the joint covariance $\mathbf{C}_{J}$ is then

$$
\mathbf{C}_{J}=\left[\begin{array}{ll}
\mathrm{C}_{1} & \mathrm{C}_{12} \\
\mathrm{C}_{21} & \mathrm{C}_{2}
\end{array}\right] \geq \mathbf{0},
$$

which happens if and only if [19]

$$
\mathrm{C}_{1} \geq \mathbf{0} \wedge \mathrm{C}_{2}-\mathrm{C}_{21} \mathrm{C}_{1}^{-1} \mathrm{C}_{12} \geq \mathbf{0},
$$

or equivalently, if and only if

$$
\mathrm{C}_{2} \geq \mathbf{0} \wedge \mathrm{C}_{1}-\mathrm{C}_{12} \mathrm{C}_{2}^{-1} \mathrm{C}_{21} \geq \mathbf{0} .
$$

It will always be assumed $\mathbf{C}_{\imath}>\mathbf{0}$ for $\imath=1, \ldots, N$. However, for the joint covariance it might be the case that $\mathbf{C}_{J} \geq \mathbf{0}$ must be assumed, e.g., if $\mathbf{C}_{1}=\mathbf{C}_{2}=\mathbf{C}_{12}=\mathbf{C}$ and hence

$$
\mathrm{C}_{J}=\left[\begin{array}{ll}
\mathrm{C} & \mathrm{C} \\
\mathrm{C} & \mathrm{C}
\end{array}\right] .
$$

\subsubsection{Covariance Intersection}

The CLUE approach to the problem of fusion under completely unknown crosscorrelations, in case of $N=2$, can be formulated as the optimization problem

$$
\begin{aligned}
\mathbf{K}_{J}^{*}, \overline{\mathbf{C}}_{J}^{*}=\underset{\substack{\mathbf{K}_{J}, \overline{\mathbf{C}}_{J} \\
\text { subject to }}}{\arg \min } & \mathbf{P} \mathbf{K}_{J} \mathbf{H}_{J}=\mathbf{I} \\
& \mathbf{P}=\mathbf{K}_{J} \overline{\mathbf{C}}_{J} \mathbf{K}_{J}^{\top}, \overline{\mathbf{C}}_{J} \in \overline{\mathbb{C}},
\end{aligned}
$$

where

$$
\begin{aligned}
& \overline{\mathbb{C}}=\left\{\overline{\mathbf{C}}_{J}=\left[\begin{array}{cc}
\overline{\mathbf{C}}_{1} & \mathbf{0} \\
\mathbf{0} & \overline{\mathrm{C}}_{2}
\end{array}\right] \mid \overline{\mathbf{C}}_{J} \geq \mathbf{C}_{J}^{\prime}, \forall \mathbf{C}_{J}^{\prime} \in \mathbb{C}\right\}, \\
& \mathbb{C}=\left\{\mathbf{C}_{J}^{\prime}=\left[\begin{array}{cc}
\mathrm{C}_{1}^{\prime} & \mathrm{C}_{12}^{\prime} \\
\mathrm{C}_{21}^{\prime} & \mathrm{C}_{2}^{\prime}
\end{array}\right] \mid \mathbf{C}_{1}^{\prime} \leq \mathbf{C}_{1}, \mathbf{C}_{2}^{\prime} \leq \mathbf{C}_{2}, \mathbf{C}_{J}^{\prime} \geq \mathbf{0}\right\} .
\end{aligned}
$$




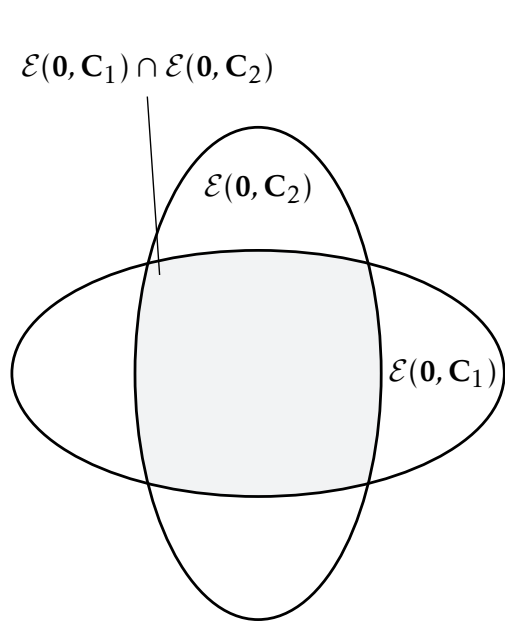

(a) Fusion of two estimates with arbitrary cross-covariance. Irrespective of $\mathbf{C}_{12}$, the ellipsoid of $\left(\mathbf{H}^{\top} \mathbf{C}_{J}^{-1} \mathbf{H}\right)^{-1}$ will lie in the intersection $\mathcal{E}\left(\mathbf{0}, \mathbf{C}_{1}\right) \cap \mathcal{E}\left(\mathbf{0}, \mathbf{C}_{2}\right)$.

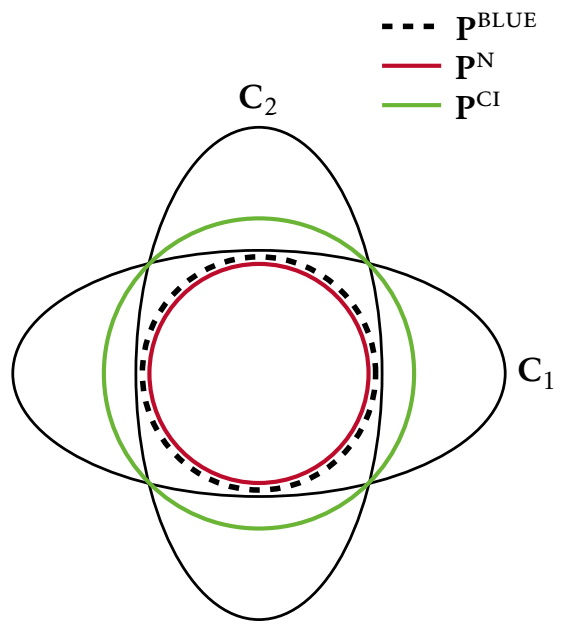

(b) Fusion of two estimates using CI. The ellipsoid $\mathcal{E}\left(\mathbf{0}, \mathbf{P}^{\mathrm{CI}}\right)$ encloses the intersection $\mathcal{E}\left(\mathbf{0}, \mathbf{C}_{1}\right) \cap \mathcal{E}\left(\mathbf{0}, \mathbf{C}_{2}\right)$. $\mathbf{P}^{\mathrm{N}}$ is the covariance calculated by a naïve estimator.

Figure 4.2: Covariance intersection.

As will be seen, the covariance intersection (CI) method presented below is a solution to (4.13).

Julier and Uhlmann published ${ }^{2}$ the CI algorithm in 1997 as a robust alternative for fusing cross-correlated estimates [22]. Two estimates $\hat{\mathbf{z}}_{1}$ and $\hat{\mathbf{z}}_{2}$ are fused using $\mathrm{CI}$ according to

$$
\begin{aligned}
\mathbf{P}^{-1} & =\omega \mathbf{C}_{1}^{-1}+(1-\omega) \mathbf{C}_{2}^{-1}, \\
\mathbf{P}^{-1} \hat{\mathbf{x}} & =\omega \mathbf{C}_{1}^{-1} \hat{\mathbf{z}}_{1}+(1-\omega) \mathbf{C}_{2}^{-1} \hat{\mathbf{z}}_{2},
\end{aligned}
$$

where the parameter $\omega \in[0,1]$ is found by optimizing $J(\mathbf{P})$, with $J(\cdot)$ being a matrix loss function.

The information form of (4.15) is illustrative-the convex combination of information implied by $\omega$ simply prohibits any information from being double counted. For example, consider an extreme case of maximum possible correlation where $\mathbf{C}_{1}=\mathbf{C}_{2}=\mathbf{C}$ and $\mathbf{C}_{12}=\mathbf{C}$. Then $\mathbf{P}^{-1}=\omega \mathbf{C}_{1}^{-1}+(1-\omega) \mathbf{C}_{2}^{-1}=\mathbf{C}^{-1}, \forall \omega \in[0,1]$ and no information has been counted twice in $\mathbf{P}^{-1}$.

In Figure 4.2a a fundamental motivation for $\mathrm{CI}$ is given. Given the fusion of

\footnotetext{
${ }^{2}$ The ideas behind the method was published already in [66], but without the name covariance intersection.
} 
$\hat{\mathbf{z}}_{1}$ and $\hat{\mathbf{z}}_{2}$ with a covariance structure described by

$$
\mathbf{C}_{J}=\left[\begin{array}{cc}
\mathbf{C}_{1} & \mathbf{C}_{12} \\
\mathbf{C}_{12}^{\top} & \mathbf{C}_{2}
\end{array}\right] \geq \mathbf{0},
$$

using the BLUE estimator the true covariance $\operatorname{cov}(\hat{\mathbf{x}})$ ellipsoid, despite the actual value of $\mathbf{C}_{12}$, will lie in the intersection of the concentric ellipsoids of $\mathbf{C}_{1}$ and $\mathbf{C}_{2}$ [23]. This is what we actually saw in Example 3.4.

Using set notation this insight can be formulated as

$$
\mathcal{E}(\mathbf{0}, \operatorname{cov}(\hat{\mathbf{x}})) \subseteq \mathcal{E}\left(\mathbf{0}, \mathbf{C}_{1}\right) \cap \mathcal{E}\left(\mathbf{0}, \mathbf{C}_{2}\right)
$$

Now, as Kahan has shown $[24,25]$, the intersection $\mathcal{E}\left(\mathbf{0}, \mathbf{C}_{1}\right) \cap \mathcal{E}\left(\mathbf{0}, \mathbf{C}_{2}\right)$ will satisfy

$$
\mathcal{E}\left(\mathbf{0}, \mathbf{C}_{1}\right) \cap \mathcal{E}\left(\mathbf{0}, \mathbf{C}_{2}\right) \subseteq \mathcal{E}\left(\mathbf{0},\left(\omega \mathbf{C}_{1}^{-1}+(1-\omega) \mathbf{C}_{2}^{-1}\right)^{-1}\right), \quad \forall \omega \in[0,1]
$$

which is exactly the same parametrization as suggested in (4.15). Hence

$$
\mathcal{E}(\mathbf{0}, \operatorname{cov}(\hat{\mathbf{x}})) \subseteq \mathcal{E}\left(\mathbf{0},\left(\omega \mathbf{C}_{1}^{-1}+(1-\omega) \mathbf{C}_{2}^{-1}\right)^{-1}\right)
$$

Kahan [24], used the notion tight to describe an ellipsoid, parametrized as

$$
\mathcal{E}\left(\mathbf{0},\left(\omega \mathbf{C}_{1}^{-1}+(1-\omega) \mathbf{C}_{2}^{-1}\right)^{-1}\right)
$$

where $\omega \in[0,1]$, that encloses the intersection $\mathcal{E}\left(\mathbf{0}, \mathbf{C}_{1}\right) \cap \mathcal{E}\left(\mathbf{0}, \mathbf{C}_{2}\right)$ such that no other ellipsoid can be squeezed in between

$$
\mathcal{E}\left(\mathbf{0}, \mathbf{C}_{1}\right) \cap \mathcal{E}\left(\mathbf{0}, \mathbf{C}_{2}\right),
$$

and

$$
\mathcal{E}\left(\mathbf{0},\left(\omega \mathbf{C}_{1}^{-1}+(1-\omega) \mathbf{C}_{2}^{-1}\right)^{-1}\right)
$$

The CI method has been generalized for the fusion of $N$ cross-correlated partial estimates, where the fused estimate and its covariance is provided in Algorithm 4.1 [22].

As long as the input estimates are conservative according to (3.18), CI yields conservatively fused estimates no matter the actual degree of correlation between the input estimates [22]. Figure 4.2b illustrates the covariance ellipse resulting from fusing $\mathbf{C}_{1}$ and $\mathbf{C}_{2}$ using $\mathrm{CI}$.

Extensive research on the $\mathrm{CI}$ method has been performed. In $[9,10] \mathrm{CI}$ is suggested as a special case of estimation under unknown correlations. A fast CI algorithm based on trace weighting is proposed in [45]. In [14] the latter was further developed. Analytical solutions to the weight $\omega$ in case of trace and determinant optimization are given in [56]. 


\section{Algorithm 4.1: Covariance Intersection General Form}

Input: $\hat{\mathbf{z}}_{1}, \ldots, \hat{\mathbf{z}}_{N}, \mathbf{C}_{1}, \ldots, \mathbf{C}_{N}, \mathbf{H}_{1}, \ldots, \mathbf{H}_{N}$

The estimates are fused according to:

$$
\begin{aligned}
\mathbf{P}^{-1} & =\sum_{\imath=1}^{N} \omega_{\imath} \mathbf{H}_{\imath}^{\top} \mathbf{C}_{\imath}^{-1} \mathbf{H}_{\imath}, \\
\mathbf{P}^{-1} \hat{\mathbf{x}} & =\sum_{\imath=1}^{N} \omega_{\imath} \mathbf{H}_{\imath}^{\top} \mathbf{C}_{\imath}^{-1} \hat{\mathbf{z}}_{\imath},
\end{aligned}
$$

where the parameters $\omega_{l} \in[0,1]$ satisfying $\sum_{l=1}^{N} \omega_{l}=1$ are found by minimizing a matrix loss function of $\mathbf{P}$.

Output: $\hat{\mathbf{x}}, \mathbf{P}$

\section{Properties}

Theorem 4.1 shows that CI can be written on WLS form, where the joint covariance $\mathrm{C}_{J}^{\mathrm{CI}}$ is given by

$$
\mathbf{C}_{J}^{\mathrm{CI}}=\left[\begin{array}{cccc}
\omega_{1}^{-1} \mathbf{C}_{1} & \mathbf{0} & \ldots & \mathbf{0} \\
\mathbf{0} & \omega_{2}^{-1} \mathbf{C}_{2} & \ddots & \vdots \\
\vdots & \ddots & \ddots & \mathbf{0} \\
\mathbf{0} & \ldots & \mathbf{0} & \omega_{N}^{-1} \mathbf{C}_{N}
\end{array}\right]
$$

which is a parametrized conservative bound. Hence, $C_{J}^{C I}=\overline{\mathbf{C}}(\omega)$ satisfies

$$
\mathbf{C}_{J}^{C I} \geq \mathbf{C}_{J} \geq \operatorname{cov}\left(\hat{\mathbf{z}}_{J}\right)
$$

where $\omega=\left[\begin{array}{lll}\omega_{1} & \ldots & \omega_{N}\end{array}\right]$.

In Theorem 4.2 it is shown that CI is a restricted best CLUE for $N=2$ in case of completely arbitrary cross-correlations.

Theorem 4.1 (Covariance Intersection-Weighted Least Squares). The covariance intersection algorithm for the fusion of $N$ estimates $\hat{\mathbf{z}}_{\imath}=\mathbf{H}_{l} \mathbf{x}+\mathbf{e}_{\imath}$, where $\imath=1, \ldots, N$, can be written as a weighted least squares estimator, with joint covariance

$$
\mathbf{C}_{J}^{\mathrm{CI}}=\left[\begin{array}{cccc}
\omega_{1}^{-1} \mathbf{C}_{1} & \mathbf{0} & \cdots & \mathbf{0} \\
\mathbf{0} & \omega_{2}^{-1} \mathbf{C}_{2} & \ddots & \vdots \\
\vdots & \ddots & \ddots & \mathbf{0} \\
\mathbf{0} & \cdots & \mathbf{0} & \omega_{N}^{-1} \mathbf{C}_{N}
\end{array}\right]
$$

and modified weight matrix $\left(\mathbf{C}_{J}^{\mathrm{CI}}\right)^{-1}$, where $\sum_{l=1}^{N} \omega_{\imath}=1$ and $\omega_{\imath} \in[0,1]$. 
Proof: Let $\mathbf{H}_{J}=\operatorname{col}\left(\mathbf{H}_{1}, \ldots, \mathbf{H}_{N}\right)$ and $\hat{\mathbf{z}}_{J}=\operatorname{col}\left(\hat{\mathbf{z}}_{1}, \ldots, \hat{\mathbf{z}}_{N}\right)$. Assume the joint covariance

$$
\mathbf{C}_{J}^{\mathrm{CI}}=\left[\begin{array}{cccc}
\omega_{1}^{-1} \mathbf{C}_{1} & \mathbf{0} & \ldots & \mathbf{0} \\
\mathbf{0} & \omega_{2}^{-1} \mathbf{C}_{2} & \ddots & \vdots \\
\vdots & \ddots & \ddots & \mathbf{0} \\
\mathbf{0} & \cdots & \mathbf{0} & \omega_{N}^{-1} \mathbf{C}_{N}
\end{array}\right]
$$

where $\sum_{l=1}^{N} \omega_{\imath}=1$ and $\omega_{\imath} \in[0,1]$, and where care should be taken if $\omega_{\imath} \in\{0,1\}$ at this point to avoid singularities. Using the WLS estimator the covariance of the fused estimate can be calculated as

$$
\mathbf{P}=\left(\mathbf{H}_{J}^{\top}\left(\mathbf{C}_{J}^{\mathrm{CI}}\right)^{-1} \mathbf{H}_{J}\right)^{-1}=\left(\sum_{l=1}^{N} \omega_{l} \mathbf{H}_{l}^{\top} \mathbf{C}_{l}^{-1} \mathbf{H}_{l}\right)^{-1},
$$

and the estimate is given by

$$
\hat{\mathbf{x}}=\left(\mathbf{H}_{J}^{\top}\left(\mathbf{C}_{J}^{\mathrm{CI}}\right)^{-1} \mathbf{H}_{J}\right)^{-1} \mathbf{H}_{J}^{\top}\left(\mathbf{C}_{J}^{\mathrm{CI}}\right)^{-1} \hat{\mathbf{z}}_{J}=\mathbf{P} \sum_{\imath=1}^{N} \omega_{\imath} \mathbf{H}_{\imath}^{\top} \mathbf{C}_{\imath}^{-1} \hat{\mathbf{z}}_{\imath},
$$

which is equivalent to the CI algorithm. Hence, CI can be expressed on WLS form with joint covariance $\mathbf{C}_{J}^{\mathrm{CI}}$ and weight matrix $\left(\mathbf{C}_{J}^{\mathrm{CI}}\right)^{-1}$.

Corollary 4.1 (Covariance Intersection-Linear Unbiased). The covariance intersection algorithm is a linear unbiased estimator.

Proof: Since the WLS estimator is linear unbiased it follows directly from Theorem 4.1 that also the CI method is a linear unbiased estimator, given that the input estimates are unbiased.

Theorem 4.2 (Covariance Intersection-Optimal). Let $\hat{\mathbf{z}}_{1}=\mathbf{H}_{1} \mathbf{x}+\mathbf{e}_{1}$ and $\hat{\mathbf{z}}_{2}=$ $\mathbf{H}_{2} \mathbf{x}+\mathbf{e}_{2}$ be given, where $\mathbf{C}_{1} \geq \operatorname{cov}\left(\hat{\mathbf{z}}_{1}\right)$ and $\mathbf{C}_{2} \geq \operatorname{cov}\left(\hat{\mathbf{z}}_{2}\right)$ are known. Then covariance intersection optimally solves (4.13) for fusion of $\hat{\mathbf{z}}_{1}$ and $\hat{\mathbf{z}}_{2}$ and hence is a restricted best conservative linear unbiased estimator.

Proof: Theorem 3.2 enables us to find a restricted best CLUE by finding an MCB of the joint covariance and then using the WLS formula (3.35). Hence, it will be shown that if there exists another conservative bound $\overline{\mathbf{C}}_{J} \geq \mathbf{C}_{J}$ then

$$
\mathrm{C}_{J}^{\mathrm{CI}} \geq \overline{\mathbf{C}}_{J} \geq \mathbf{0} \Longrightarrow \overline{\mathbf{C}}_{J}=\mathrm{C}_{J}^{\mathrm{CI}} \text {. }
$$

Assuming $\mathbf{C}_{J} \geq \mathbf{0}$ is equivalent to [19]

$$
\mathrm{C}_{1} \geq \mathbf{0} \wedge \quad \mathrm{C}_{1} \geq \mathrm{C}_{12} \mathrm{C}_{2}^{-1} \mathrm{C}_{21} .
$$


The joint covariance $\mathbf{C}_{J}^{\mathrm{CI}}=\mathbf{C}_{J}^{\mathrm{CI}}(\omega)$ is a conservative bound, parametrized by $\omega$, on $\mathrm{C}_{J}$ if and only if

$$
\mathbf{C}_{J}^{\mathrm{CI}}-\mathbf{C}_{J}=\left[\begin{array}{cc}
\frac{\mathbf{C}_{1}}{\omega} & \mathbf{0} \\
\mathbf{0} & \frac{\mathbf{C}_{2}}{1-\omega}
\end{array}\right]-\left[\begin{array}{cc}
\mathbf{C}_{1} & \mathbf{C}_{12} \\
\mathbf{C}_{21} & \mathbf{C}_{2}
\end{array}\right]=\left[\begin{array}{cc}
\frac{\mathbf{C}_{1}}{\omega}-\mathbf{C}_{1} & -\mathbf{C}_{12} \\
-\mathbf{C}_{21} & \frac{\mathbf{C}_{2}}{1-\omega}-\mathbf{C}_{2}
\end{array}\right] \geq \mathbf{0},
$$

for all $\omega \in(0,1)$. The cases with $\omega \in\{0,1\}$ are handled trivially since then either of the covariances $\mathbf{C}_{1}$ or $\mathbf{C}_{2}$ is chosen exclusively. $\mathbf{C}_{J}^{\mathrm{CI}}-\mathbf{C}_{J} \geq \mathbf{0}$ is true if and only if [19]

$$
\frac{\mathbf{C}_{1}}{\omega}-\mathbf{C}_{1} \geq \mathbf{0}
$$

which is true since $\left(\frac{1}{\omega}-1\right) \mathbf{C}_{1} \geq \mathbf{0}$, and

$$
\begin{aligned}
& \frac{\mathbf{C}_{1}}{\omega}-\mathbf{C}_{1} \geq \mathbf{C}_{12}\left(\frac{1}{1-\omega} \mathbf{C}_{2}-\mathbf{C}_{2}\right)^{-1} \mathbf{C}_{21} \\
\Longleftrightarrow & \frac{1-\omega}{\omega} \mathbf{C}_{1} \geq \mathbf{C}_{12}\left(\frac{\omega}{1-\omega} \mathbf{C}_{2}\right)^{-1} \mathbf{C}_{21} \\
\Longleftrightarrow & \mathbf{C}_{1} \geq \mathbf{C}_{12} \mathbf{C}_{2}^{-1} \mathbf{C}_{21},
\end{aligned}
$$

which follows from (4.24).

Assume there exists another bound $\overline{\mathbf{C}}_{J}=\operatorname{diag}\left(\overline{\mathbf{C}}_{1}, \overline{\mathbf{C}}_{2}\right)$ where $\overline{\mathbf{C}}_{1}>\mathbf{C}_{1}$ and $\overline{\mathbf{C}}_{2}>\mathbf{C}_{2}$, which satisfies $\mathbf{C}_{J}^{\mathrm{CI}} \geq \overline{\mathbf{C}}_{J} \geq \mathbf{C}_{J}$ and

$$
\begin{aligned}
\frac{\mathbf{C}_{1}}{\omega} & \geq \overline{\mathbf{C}}_{1}>\mathbf{C}_{1}, \\
\frac{\mathbf{C}_{2}}{1-\omega} & \geq \overline{\mathbf{C}}_{2}>\mathbf{C}_{2} .
\end{aligned}
$$

A consequence of the assumptions above is

$$
\begin{aligned}
\frac{\mathbf{C}_{1}}{\omega}-\mathbf{C}_{1} \geq \overline{\mathbf{C}}_{1}-\mathbf{C}_{1} & \Longleftrightarrow\left(\frac{\mathbf{C}_{1}}{\omega}-\mathbf{C}_{1}\right)^{-1} \leq\left(\overline{\mathbf{C}}_{1}-\mathbf{C}_{1}\right)^{-1}, \\
\frac{\mathbf{C}_{2}}{1-\omega}-\mathbf{C}_{2} \geq \overline{\mathbf{C}}_{2}-\mathbf{C}_{2} & \Longleftrightarrow\left(\frac{\mathbf{C}_{2}}{1-\omega}-\mathbf{C}_{2}\right)^{-1} \leq\left(\overline{\mathbf{C}}_{2}-\mathbf{C}_{2}\right)^{-1},
\end{aligned}
$$

where $\mathbf{A}_{1} \geq \mathbf{A}_{2} \Longleftrightarrow \mathbf{A}_{2}^{-1} \geq \mathbf{A}_{1}^{-1}$ for PD matrices $\mathbf{A}_{1}$ and $\mathbf{A}_{2}$ has been used. For $\overline{\mathbf{C}}_{J}-\mathbf{C}_{J} \geq \mathbf{0}$ to hold

$$
\overline{\mathbf{C}}_{1}-\mathbf{C}_{1} \geq \mathbf{C}_{12}\left(\overline{\mathbf{C}}_{2}-\mathbf{C}_{2}\right)^{-1} \mathbf{C}_{21} \geq \mathbf{C}_{12}\left(\frac{\mathbf{C}_{2}}{1-\omega}-\mathbf{C}_{2}\right)^{-1} \mathbf{C}_{21}=\mathbf{C}_{12}\left(\frac{\omega}{1-\omega} \mathbf{C}_{2}\right)^{-1} \mathbf{C}_{21} \text {, }
$$

where (4.26b) has been used. Using (4.24) we get

$$
\overline{\mathbf{C}}_{1} \geq \mathbf{C}_{12} \mathbf{C}_{2}^{-1} \mathbf{C}_{21}+\mathbf{C}_{12}\left(\frac{\omega}{1-\omega} \mathbf{C}_{2}\right)^{-1} \mathbf{C}_{21}=\mathbf{C}_{12}\left(\frac{1-\omega+\omega}{\omega}\right) \mathbf{C}_{2}^{-1} \mathbf{C}_{21}=\mathbf{C}_{12} \frac{\mathbf{C}_{2}^{-1}}{\omega} \mathbf{C}_{21} \text {, }
$$


i.e., $\omega \overline{\mathbf{C}}_{1} \geq \mathbf{C}_{12} \mathbf{C}_{2}^{-1} \mathbf{C}_{21}$. Since $\mathbf{C}_{1} \geq \mathbf{C}_{12} \mathbf{C}_{2}^{-1} \mathbf{C}_{21}$ and $\overline{\mathbf{C}}_{1} \geq \mathbf{C}_{1}$ it must also be required that $\overline{\mathbf{C}}_{1} \geq \frac{\mathbf{C}_{1}}{\omega}$. Hence, the assumption in (4.25a) implies

$$
\frac{\mathbf{C}_{1}}{\omega} \geq \overline{\mathbf{C}}_{1} \geq \frac{\mathbf{C}_{1}}{\omega},
$$

which can only be fulfilled if $\overline{\mathbf{C}}_{1}=\frac{\mathrm{C}_{1}}{\omega}$. Using a similar reasoning it can also be shown $\overline{\mathbf{C}}_{2}=\frac{\mathbf{C}_{2}}{1-\omega}$. Hence, $\mathbf{C}_{J}^{\mathrm{CI}}$ is an $\mathrm{MCB}$ of $\mathbf{C}_{J}$. By Theorem 3.2 and Theorem 4.1 it then follows that CI optimally solves (4.13).

The results of Theorem 4.2 are shown somewhat differently in [58] in case of $N=2$ and $\mathbf{H}_{1}=\mathbf{H}_{2}=\mathbf{I}$. In fact, the optimality proof of [58] also proves that $\mathrm{CI}$ is the solution to (3.12), i.e., that CI is a best CLUE in case of completely unknown cross-correlations, a possibly stronger statement. Theorem 4.2 is however valid for arbitrary $\mathbf{H}_{1}$ and $\mathbf{H}_{2}$ as long as $\operatorname{rank}\left(\left[\begin{array}{ll}\mathbf{H}_{1}^{\top} & \mathbf{H}_{2}^{\top}\end{array}\right]\right)=n_{x}$, since the latter is assumed in Theorem 3.2 (which is used in the proof of Theorem 4.2).

\subsection{Fusion Under Correlated Information}

The cross-correlation structure called correlated information is defined, followed by a presentation and an analysis of the ICI method.

\subsubsection{Correlated Information}

The correlated information structure is realized in setups where multiple estimates at one point are cross-correlated to an unknown degree followed by new uncorrelated information $\left(\mathbf{C}_{l}^{e}\right)^{-1}$, typically derived from new measurements, being added to each of the estimates.

Before defining the correlated information structure the closely related common information structure is given. Two estimates $\hat{\mathbf{z}}_{1}$ and $\hat{\mathbf{z}}_{2}$ can be decomposed according to the common information structure if for $\imath=1,2$

$$
\begin{aligned}
\mathbf{C}_{l}^{-1} & =\left(\mathbf{C}_{l}^{e}\right)^{-1}+\Gamma^{-1}, \\
\mathbf{C}_{l}^{-1} \hat{\mathbf{z}}_{l} & =\left(\mathbf{C}_{l}^{e}\right)^{-1} \hat{\mathbf{z}}_{l}^{e}+\Gamma^{-1} \hat{\gamma},
\end{aligned}
$$

is satisfied, where $\Gamma^{-1}$ is the common information shared by the two estimates and $\hat{\gamma}$ is the corresponding estimate, and $\left(\mathbf{C}_{l}^{e}\right)^{-1}$ is referred to as exclusive information related to the estimates $\hat{\mathbf{z}}_{l}^{e}$, i.e., $\mathbf{C}_{l}^{e}=\operatorname{cov}\left(\hat{\mathbf{z}}_{l}^{e}\right)$. The decomposition in (4.27) is for example realized in a situation where two estimates are initiated with $\hat{\gamma}$ and $\Gamma$ after which exclusive information is being added to each of the estimates.

The correlated information structure is a generalization of the common information structure. Two estimates $\hat{\mathbf{z}}_{1}$ and $\hat{\mathbf{z}}_{2}$ can be decomposed according to the common information structure if [48]

$$
\begin{aligned}
\mathbf{C}_{\imath}^{-1} & =\left(\mathbf{C}_{\imath}^{e}\right)^{-1}+\Gamma_{\imath}^{-1}, \\
\mathbf{C}_{\imath}^{-1} \hat{\mathbf{z}}_{\imath} & =\left(\mathbf{C}_{\imath}^{e}\right)^{-1} \hat{\mathbf{z}}_{\imath}^{e}+\Gamma_{\imath}^{-1} \hat{\gamma}_{\imath},
\end{aligned}
$$


hold for $\imath=1,2$, where in general $\hat{\gamma}_{1} \neq \hat{\gamma}_{2}$ and $\Gamma_{1} \neq \Gamma_{2}$. The covariance of $\hat{\gamma}_{J}=$ $\left[\begin{array}{ll}\hat{\gamma}_{1}^{\top} & \hat{\gamma}_{2}^{\top}\end{array}\right]^{\top}$ is given by

$$
\Gamma_{J}=\left[\begin{array}{cc}
\Gamma_{1} & \Gamma_{12} \\
\Gamma_{21} & \Gamma_{2}
\end{array}\right] .
$$

It is further assumed $\operatorname{cov}\left(\hat{\mathbf{z}}_{1}^{e}, \hat{\gamma}_{1}\right)=\mathbf{0}, \operatorname{cov}\left(\hat{\mathbf{z}}_{2}^{e}, \hat{\gamma}_{2}\right)=\mathbf{0}$ and $\operatorname{cov}\left(\hat{\mathbf{z}}_{1}^{e}, \hat{\mathbf{z}}_{2}^{e}\right)=\operatorname{cov}\left(\hat{\mathbf{z}}_{1}^{e}, \hat{\gamma}_{2}\right)=$ $\operatorname{cov}\left(\hat{\gamma}_{1}, \hat{\mathbf{z}}_{2}^{e}\right)=\mathbf{0}$ such that

$$
\operatorname{cov}\left(\left[\begin{array}{c}
\hat{\mathbf{z}}_{1}^{e} \\
\hat{\gamma}_{1} \\
\hat{\mathbf{z}}_{2}^{e} \\
\hat{\gamma}_{2}
\end{array}\right]\right)=\left[\begin{array}{cccc}
\mathbf{C}_{1}^{e} & \mathbf{0} & \mathbf{0} & \mathbf{0} \\
\mathbf{0} & \boldsymbol{\Gamma}_{1} & \mathbf{0} & \boldsymbol{\Gamma}_{12} \\
\mathbf{0} & \mathbf{0} & \mathbf{C}_{2}^{e} & \mathbf{0} \\
\mathbf{0} & \boldsymbol{\Gamma}_{21} & \mathbf{0} & \boldsymbol{\Gamma}_{2}
\end{array}\right]
$$

Since $\mathrm{E} \hat{\mathbf{z}}_{\imath}=\mathrm{E} \hat{\gamma}_{\imath}=\mathbf{H}_{\imath} \mathbf{x}$, and by using (4.28) and (4.30) it follows that

$$
\begin{aligned}
\operatorname{cov}\left(\hat{\mathbf{z}}_{1}, \hat{\mathbf{z}}_{2}\right) & =\mathrm{E} \tilde{\mathbf{z}}_{1} \tilde{\mathbf{z}}_{2}^{\top} \\
& =\mathrm{E}\left(\mathbf{C}_{1}\left(\left(\mathbf{C}_{1}^{e}\right)^{-1} \hat{\mathbf{z}}_{1}^{e}+\Gamma_{1}^{-1} \hat{\gamma}_{1}\right)-\mathbf{H}_{1} \mathbf{x}\right) \tilde{\mathbf{z}}_{2}^{\top} \\
& =\mathrm{E}\left(\mathbf{C}_{1}\left(\left(\mathbf{C}_{1}^{e}\right)^{-1} \hat{\mathbf{z}}_{1}^{e}+\Gamma_{1}^{-1} \hat{\gamma}_{1}\right)-\mathbf{C}_{1}\left(\left(\mathbf{C}_{1}^{e}\right)^{-1}+\boldsymbol{\Gamma}_{1}^{-1}\right) \mathbf{H}_{1} \mathbf{x}\right) \tilde{\mathbf{z}}_{2}^{\top} \\
& =\mathrm{E} \mathbf{C}_{1}\left(\left(\mathbf{C}_{1}^{e}\right)^{-1} \tilde{\mathbf{z}}_{1}^{e}+\Gamma_{1}^{-1} \tilde{\gamma}_{1}\right) \tilde{\mathbf{z}}_{2}^{\top} \\
& =\mathrm{E} \mathbf{C}_{1}\left(\left(\mathbf{C}_{1}^{e}\right)^{-1} \tilde{\mathbf{z}}_{1}^{e}+\boldsymbol{\Gamma}_{1}^{-1} \tilde{\gamma}_{1}\right)\left(\left(\mathbf{C}_{2}^{e}\right)^{-1} \tilde{\mathbf{z}}_{2}^{e}+\boldsymbol{\Gamma}_{2}^{-1} \tilde{\gamma}_{2}\right)^{\top} \mathbf{C}_{2} \\
& =\mathbf{C}_{1} \boldsymbol{\Gamma}_{1}^{-1} \mathrm{E} \tilde{\gamma}_{1} \tilde{\gamma}_{2}^{\top} \Gamma_{2}^{-1} \mathbf{C}_{2} \\
& =\mathbf{C}_{1} \boldsymbol{\Gamma}_{1}^{-1} \boldsymbol{\Gamma}_{12} \boldsymbol{\Gamma}_{2}^{-1} \mathbf{C}_{2} .
\end{aligned}
$$

The common information structure is recovered from the correlated information structure by letting $\hat{\gamma}_{1}=\hat{\gamma}_{2}=\hat{\gamma}$ and $\Gamma_{1}=\Gamma_{2}=\Gamma$, which yield

$$
\Gamma_{J}=\left[\begin{array}{ll}
\Gamma & \Gamma \\
\Gamma & \Gamma
\end{array}\right] .
$$

Common information is also recognized as shared information.

\subsubsection{Inverse Covariance Intersection}

The CLUE approach to the problem of fusion under correlated information, in case of $N=2$, can be formulated as the optimization problem

$$
\begin{aligned}
\mathbf{K}_{J}^{*}, \overline{\mathbf{C}}_{J}^{*}=\underset{\substack{\mathbf{K}_{J}, \overline{\mathbf{C}}_{J} \\
\text { subject to }}}{\arg \min } & \mathbf{P} \\
& \mathbf{P}=\mathbf{K}_{J} \mathbf{H}_{J}=\mathbf{I} \\
&
\end{aligned}
$$


where

$$
\begin{aligned}
& \overline{\mathbb{C}}=\left\{\overline{\mathbf{C}}_{J}=\left[\begin{array}{cc}
\overline{\mathbf{C}}_{1} & \mathbf{0} \\
\mathbf{0} & \overline{\mathbf{C}}_{2}
\end{array}\right] \mid \overline{\mathbf{C}}_{J} \geq \mathbf{C}_{J}^{\prime}, \forall \mathbf{C}_{J}^{\prime} \in \mathbb{C}\right\}, \\
& \mathbb{C}=\left\{\begin{array}{l|l}
\mathbf{C}_{J}^{\prime}=\left[\begin{array}{cc}
\mathbf{C}_{1}^{\prime} & \mathbf{C}_{12}^{\prime} \\
\mathbf{C}_{21}^{\prime} & \mathbf{C}_{2}^{\prime}
\end{array}\right] & \begin{array}{l}
\left(\mathbf{C}_{1}^{\prime}\right)^{-1} \geq \mathbf{C}_{1}^{-1}=\left(\mathbf{C}_{1}^{e}\right)^{-1}+\Gamma_{1}^{-1} \\
\left(\mathbf{C}_{2}^{\prime}\right)^{-1} \geq \mathbf{C}_{2}^{-1}=\left(\mathbf{C}_{2}^{e}\right)^{-1}+\Gamma_{2}^{-1} \\
\exists \alpha>0, \mathbf{C}_{1}^{-1} \geq \frac{1}{\alpha} \boldsymbol{\Gamma}_{2}^{-1}, \mathbf{C}_{2}^{-1} \geq \alpha \Gamma_{1}^{-1} \\
\mathbf{C}_{J}^{\prime} \geq \mathbf{0}, \mathbf{\Gamma}_{J} \geq \mathbf{0}, \mathbf{\Gamma}_{J}=\left[\begin{array}{cc}
\mathbf{\Gamma}_{1} & \boldsymbol{\Gamma}_{12} \\
\mathbf{\Gamma}_{21} & \boldsymbol{\Gamma}_{2}
\end{array}\right]
\end{array}
\end{array}\right\} .
\end{aligned}
$$

As will be seen, the inverse covariance intersection (ICI) method presented next is a solution to (4.33), which is algebraically equivalent to (4.13) for the CI case. Due to the new assumptions on the cross-correlations the set $\mathbb{C}_{J}$ now generally includes fewer elements. Hence, $\overline{\mathbb{C}}_{J}$ now might include elements $\overline{\mathbf{C}}_{J}$ that are smaller, in the PSD sense, compared to all elements of $\overline{\mathbb{C}}$ in the CI case.

The ICI method is a less conservative alternative to CI and was developed by Noack et al in 2017 [49]. In its original form, ICI assumes that two estimates $\hat{\mathbf{z}}_{1}$ and $\hat{\mathbf{z}}_{2}$, where $\mathbf{H}_{1}=\mathbf{H}_{2}=\mathbf{I}$, to be fused can be decomposed according to the common information decomposition presented in Section 4.2.1. The ICI fusion rule is given by

$$
\begin{aligned}
\mathbf{P}^{-1}= & \mathbf{C}_{1}^{-1}+\mathbf{C}_{2}^{-1}-\left(\omega \mathbf{C}_{1}+(1-\omega) \mathbf{C}_{2}\right)^{-1}, \\
\mathbf{P}^{-1} \hat{\mathbf{x}}= & \left(\mathbf{C}_{1}^{-1}-\omega\left(\omega \mathbf{C}_{1}+(1-\omega) \mathbf{C}_{2}\right)^{-1}\right) \hat{\mathbf{z}}_{1} \\
& +\left(\mathbf{C}_{2}^{-1}-(1-\omega)\left(\omega \mathbf{C}_{1}+(1-\omega) \mathbf{C}_{2}\right)^{-1}\right) \hat{\mathbf{z}}_{2},
\end{aligned}
$$

where $\omega \in[0,1]$ is found by optimizing $J(\mathbf{P})$. The result from fusing two estimates using ICI is illustrated in Figure 4.3b, where the covariance of the fused estimate has been denoted $\mathrm{P}^{\mathrm{ICI}}$.

The basic idea behind ICI is illustrated in Figure 4.3a. Given that the decomposition in (4.27) holds, the ICI method tries to bound the ellipsoids of all possible $\Gamma^{-1}$. While CI finds an ellipsoid that encloses the intersection of two covariance ellipsoids $\mathcal{E}\left(\mathbf{0}, \mathbf{C}_{1}\right)$ and $\mathcal{E}\left(\mathbf{0}, \mathbf{C}_{2}\right)$, ICI finds an ellipsoid that encloses the intersection of two information ellipsoids $\mathcal{E}\left(\mathbf{0}, \mathbf{C}_{1}^{-1}\right)$ and $\mathcal{E}\left(\mathbf{0}, \mathbf{C}_{2}^{-1}\right)$, which in this case is the ellipsoid given by

$$
\mathcal{E}\left(\mathbf{0},\left(\omega \mathbf{C}_{1}+(1-\omega) \mathbf{C}_{2}\right)^{-1}\right)
$$

Otherwise put, the information $\left(\omega \mathbf{C}_{1}+(1-\omega) \mathbf{C}_{2}\right)^{-1}$ is an upper bound of all possible $\boldsymbol{\Gamma}^{-1}$. The information $\left(\omega \mathbf{C}_{1}+(1-\omega) \mathbf{C}_{2}\right)^{-1}$ is exactly the information that is being subtracted from $\mathbf{C}_{1}^{-1}+\mathbf{C}_{2}^{-2}$ in (4.35a).

In [48] the ICI method is further developed and shown to be able to handle the correlated information decomposition presented in Section 4.2.1, viz.,

$$
\begin{aligned}
\mathbf{C}_{\imath}^{-1} & =\left(\mathbf{C}_{\imath}^{e}\right)^{-1}+\Gamma_{\imath}^{-1}, \\
\mathbf{C}_{\imath}^{-1} \hat{\mathbf{z}}_{\imath} & =\left(\mathbf{C}_{\imath}^{e}\right)^{-1} \hat{\mathbf{z}}_{\imath}^{e}+\Gamma_{l}^{-1} \hat{\gamma}_{\imath} .
\end{aligned}
$$




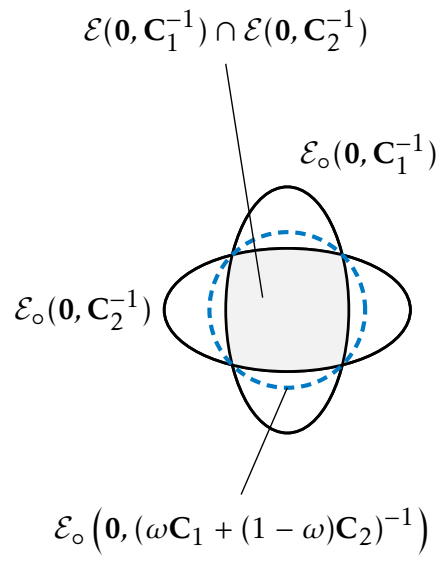

(a) In ICI the correlated information is bounded from above and then subtracted.

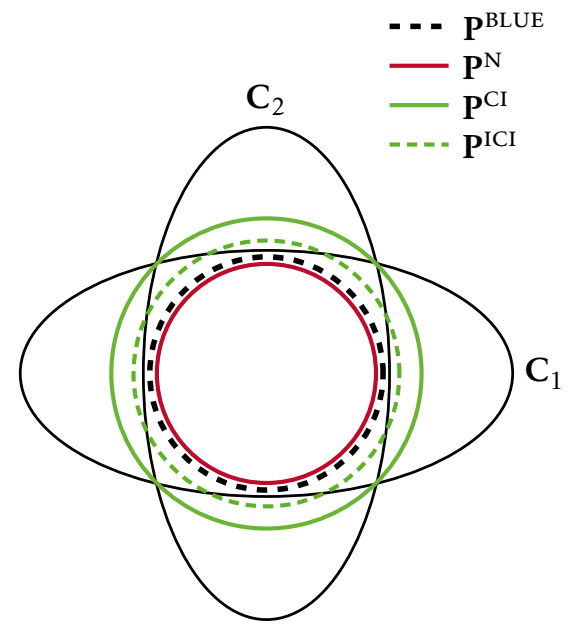

(b) Fusion of two estimates using ICI. $\mathbf{P}^{\mathrm{N}}$ is the covariance calculated by a naïve estimator.

Figure 4.3: Inverse covariance intersection.

The cross-correlations of the two estimates $\hat{\gamma}_{1}$ and $\hat{\gamma}_{2}$ are given by the off-diagonal blocks in

$$
\Gamma_{J}=\left[\begin{array}{ll}
\boldsymbol{\Gamma}_{1} & \boldsymbol{\Gamma}_{12} \\
\boldsymbol{\Gamma}_{21} & \boldsymbol{\Gamma}_{2}
\end{array}\right],
$$

which is the covariance of the joint estimate $\hat{\gamma}_{J}=\left[\begin{array}{ll}\hat{\gamma}_{1}^{\top} & \hat{\gamma}_{2}^{\top}\end{array}\right]^{\top}$.

The ICI method has been proven to provide conservative estimates if the decomposition of (4.37) holds and the following conditions are valid [48]

$$
\begin{aligned}
& \mathbf{C}_{2}^{-1} \geq \alpha \boldsymbol{\Gamma}_{1}^{-1}, \\
& \mathbf{C}_{1}^{-1} \geq \frac{1}{\alpha} \boldsymbol{\Gamma}_{2}^{-1},
\end{aligned}
$$

for some $\alpha>0$. The conditions of (4.39) might hold even if, e.g., $\Gamma_{1}^{-1} \geq \mathbf{C}_{2}$. Another interpretation is that correlated information and hence common information might only be included in one of the estimates [48].

In [1] the ICI fusion rule is extended to handle multiple estimates batchwise. To the author's knowledge, there exists no variant of ICI for the fusion of partial estimates. Hence, below follows a proposed modification of ICI to be able to handle partial estimates.

\section{Inverse Covariance Intersection for Partial Estimates}

The following generalization of ICI will be valid for the fusion of exactly two partial estimates, i.e., estimates given on the form of (2.40). The generalized 
version of ICI is abbreviated ICIP.

As before the underlying estimates $\hat{\gamma}_{1}$ and $\hat{\gamma}_{2}$ are assumed, with joint covariance given by

$$
\Gamma_{J}=\left[\begin{array}{cc}
\Gamma_{1} & \Gamma_{12} \\
\Gamma_{21} & \Gamma_{2}
\end{array}\right]
$$

The partial estimates to be fused can be decomposed as

$$
\begin{aligned}
\mathbf{C}_{l}^{-1} & =\left(\mathbf{C}_{\imath}^{e}\right)^{-1}+\mathbf{G}_{\imath}^{\top} \boldsymbol{\Gamma}_{\imath}^{-1} \mathbf{G}_{\imath}, \\
\mathbf{C}_{l}^{-1} \hat{\mathbf{z}}_{l} & =\left(\mathbf{C}_{\imath}^{e}\right)^{-1} \hat{\mathbf{z}}_{\imath}^{e}+\mathbf{G}_{\imath}^{\top} \boldsymbol{\Gamma}_{l}^{-1} \hat{\gamma}_{l},
\end{aligned}
$$

for $\imath=1,2$. The matrix $\mathbf{G}_{\imath}$ is the mapping from the space of $\hat{\mathbf{z}}_{\imath}$ to the space of $\hat{\gamma}_{\imath}$ and hence determines how correlated information $\Gamma_{\imath}^{-1}$ enters the $\imath$ th estimate. The cross-covariance of $\hat{\mathbf{z}}_{1}$ and $\hat{\mathbf{z}}_{2}$ now become

$$
\operatorname{cov}\left(\hat{\mathbf{z}}_{1}, \hat{\mathbf{z}}_{2}\right)=\mathbf{C}_{12}=\mathbf{C}_{21}^{\top}=\mathbf{C}_{1} \mathbf{G}_{1}^{\top} \boldsymbol{\Gamma}_{1}^{-1} \mathbf{G}_{1} \boldsymbol{\Gamma}_{12} \mathbf{G}_{2}^{\top} \boldsymbol{\Gamma}_{2}^{-1} \mathbf{G}_{2} \mathbf{C}_{2}
$$

The joint covariance is given by

$$
\mathbf{C}_{J}^{\mathrm{ICIP}}=\left[\begin{array}{cc}
\mathbf{C}_{1}+\frac{\omega}{1-\omega} \mathbf{C}_{1} \mathbf{H}_{1} \mathbf{H}_{2}^{\top} \mathbf{C}_{2}^{-1} \mathbf{H}_{2} \mathbf{H}_{1}^{\top} \mathbf{C}_{1} & \mathbf{0} \\
\mathbf{0} & \mathbf{C}_{2}+\frac{1-\omega}{\omega} \mathbf{C}_{2} \mathbf{H}_{2} \mathbf{H}_{1}^{\top} \mathbf{C}_{1}^{-1} \mathbf{H}_{1} \mathbf{H}_{2}^{\top} \mathbf{C}_{2}
\end{array}\right],
$$

where $\omega \in[0,1]$, with the extreme cases when $\omega \in\{0,1\}$ handled trivially since in these cases one of the input estimates is used exclusively to form the fused estimate. Using Woodbury's matrix identity [68] and the WLS formula of (3.35) the covariance of the ICIP estimate $\hat{\mathbf{x}}$ is calculated as

$$
\begin{aligned}
\mathbf{P}^{-1}= & \mathbf{H}_{J}^{\top}\left(\mathbf{C}_{J}^{\mathrm{ICIP}}\right)^{-1} \mathbf{H}_{J} \\
= & \mathbf{H}_{1}^{\top} \mathbf{C}_{1}^{-1} \mathbf{H}_{1}-\mathbf{H}_{1}^{\top} \mathbf{H}_{1} \mathbf{H}_{2}^{\top}\left(\frac{1-\omega}{\omega} \mathbf{C}_{2}+\mathbf{H}_{2} \mathbf{H}_{1}^{\top} \mathbf{C}_{1} \mathbf{H}_{1} \mathbf{H}_{2}^{\top}\right)^{-1} \mathbf{H}_{2} \mathbf{H}_{1}^{\top} \mathbf{H}_{1} \\
& +\mathbf{H}_{2}^{\top} \mathbf{C}_{2}^{-1} \mathbf{H}_{2}-\mathbf{H}_{2}^{\top} \mathbf{H}_{2} \mathbf{H}_{1}^{\top}\left(\frac{\omega}{1-\omega} \mathbf{C}_{1}+\mathbf{H}_{1} \mathbf{H}_{2}^{\top} \mathbf{C}_{2} \mathbf{H}_{2} \mathbf{H}_{1}^{\top}\right)^{-1} \mathbf{H}_{1} \mathbf{H}_{2}^{\top} \mathbf{H}_{2} \\
= & \mathbf{H}_{1}^{\top} \mathbf{C}_{1}^{-1} \mathbf{H}_{1}-\omega \mathbf{H}_{1}^{\top} \mathbf{H}_{1} \mathbf{H}_{2}^{\top}\left(\omega \mathbf{H}_{2} \mathbf{H}_{1}^{\top} \mathbf{C}_{1} \mathbf{H}_{1} \mathbf{H}_{2}^{\top}+(1-\omega) \mathbf{C}_{2}\right)^{-1} \mathbf{H}_{2} \mathbf{H}_{1}^{\top} \mathbf{H}_{1} \\
& +\mathbf{H}_{2}^{\top} \mathbf{C}_{2}^{-1} \mathbf{H}_{2}-(1-\omega) \mathbf{H}_{2}^{\top} \mathbf{H}_{2} \mathbf{H}_{1}^{\top}\left(\omega \mathbf{C}_{1}+(1-\omega) \mathbf{H}_{1} \mathbf{H}_{2}^{\top} \mathbf{C}_{2} \mathbf{H}_{2} \mathbf{H}_{1}^{\top}\right)^{-1} \mathbf{H}_{1} \mathbf{H}_{2}^{\top} \mathbf{H}_{2} .
\end{aligned}
$$

The ICIP method is given in Algorithm 4.2.

The new conditions corresponding to (4.39), specifying the amount of correlated information allowed in each estimate, are given by

$$
\begin{aligned}
& \mathbf{H}_{1} \mathbf{H}_{2}^{\top} \mathbf{C}_{2}^{-1} \mathbf{H}_{2} \mathbf{H}_{1}^{\top} \geq \alpha \mathbf{G}_{1}^{\top} \boldsymbol{\Gamma}_{1}^{-1} \mathbf{G}_{1} \boldsymbol{\Gamma}_{1} \mathbf{G}_{1}^{\top} \boldsymbol{\Gamma}_{1}^{-1} \mathbf{G}_{1}, \\
& \mathbf{H}_{2} \mathbf{H}_{1}^{\top} \mathbf{C}_{1}^{-1} \mathbf{H}_{1} \mathbf{H}_{2}^{\top} \geq \frac{1}{\alpha} \mathbf{G}_{2}^{\top} \boldsymbol{\Gamma}_{2}^{-1} \mathbf{G}_{2} \boldsymbol{\Gamma}_{2} \mathbf{G}_{2}^{\top} \boldsymbol{\Gamma}_{2}^{-1} \mathbf{G}_{2},
\end{aligned}
$$

for some $\alpha>0$. As an example of when the conditions of (4.47) fails to hold, consider a case where the matrices on the 1.h.s. and right hand side (r.h.s.) of 


\section{Algorithm 4.2: Inverse Covariance Intersection Partial Estimate Form}

Input: $\hat{\mathbf{z}}_{1}, \hat{\mathbf{z}}_{2}, \mathbf{C}_{1}, \mathbf{C}_{2}, \mathbf{H}_{1}, \mathbf{H}_{2}$

The estimates are fused according to:

$$
\begin{aligned}
\mathbf{P}^{-1}= & \mathbf{H}_{1}^{\top} \mathbf{C}_{1}^{-1} \mathbf{H}_{1}-\omega \mathbf{H}_{1}^{\top} \mathbf{H}_{1} \mathbf{H}_{2}^{\top} \mathbf{C}_{\boldsymbol{\Gamma}_{1}}^{-1} \mathbf{H}_{2} \mathbf{H}_{1}^{\top} \mathbf{H}_{1} \\
& +\mathbf{H}_{2}^{\top} \mathbf{C}_{2}^{-1} \mathbf{H}_{2}-(1-\omega) \mathbf{H}_{2}^{\top} \mathbf{H}_{2} \mathbf{H}_{1}^{\top} \mathbf{C}_{\boldsymbol{\Gamma}_{2}}^{-1} \mathbf{H}_{1} \mathbf{H}_{2}^{\top} \mathbf{H}_{2}, \\
\mathbf{P}^{-1} \hat{\mathbf{x}}= & \left(\mathbf{H}_{1}^{\top} \mathbf{C}_{1}^{-1}-\omega \mathbf{H}_{1}^{\top} \mathbf{H}_{1} \mathbf{H}_{2}^{\top} \mathbf{C}_{\boldsymbol{\Gamma}_{1}}^{-1} \mathbf{H}_{2} \mathbf{H}_{1}^{\top}\right) \hat{\mathbf{z}}_{1} \\
& +\left(\mathbf{H}_{2}^{\top} \mathbf{C}_{2}^{-1}-(1-\omega) \mathbf{H}_{2}^{\top} \mathbf{H}_{2} \mathbf{H}_{1}^{\top} \mathbf{C}_{\boldsymbol{\Gamma}_{2}}^{-1} \mathbf{H}_{1} \mathbf{H}_{2}^{\top}\right) \hat{\mathbf{z}}_{2},
\end{aligned}
$$

where

$$
\begin{aligned}
& \mathbf{C}_{\boldsymbol{\Gamma}_{1}}=\omega \mathbf{H}_{2} \mathbf{H}_{1}^{\top} \mathbf{C}_{1} \mathbf{H}_{1} \mathbf{H}_{2}^{\top}+(1-\omega) \mathbf{C}_{2}, \\
& \mathbf{C}_{\boldsymbol{\Gamma}_{2}}=\omega \mathbf{C}_{1}+(1-\omega) \mathbf{H}_{1} \mathbf{H}_{2}^{\top} \mathbf{C}_{2} \mathbf{H}_{2} \mathbf{H}_{1}^{\top} .
\end{aligned}
$$

The parameter $\omega \in[0,1]$ is found by minimizing a matrix loss function of $\mathbf{P}$. Output: $\hat{\mathbf{x}}, \mathbf{P}$

(4.47a) are respectively given by

$$
\left[\begin{array}{ll}
c & 0 \\
0 & 0
\end{array}\right], \quad \alpha\left[\begin{array}{ll}
0 & 0 \\
0 & d
\end{array}\right],
$$

where $c, d>0$. In this case (4.47a) cannot be satisfied for any $\alpha>0$.

The matrix $\mathbf{G}_{\imath}$ might not always be available. For example, say that an actor holds $\hat{\mathbf{z}}_{1}$ and $\mathbf{C}_{1}$, and receives $\hat{\mathbf{z}}_{2}$ and $\mathbf{C}_{2}$. In this case it is reasonable to assume that the actor has access to $\mathbf{G}_{1}$, since $\hat{\mathbf{z}}_{1}^{e}$ and $\mathbf{C}_{1}^{e}$ were earlier known, but $\mathbf{G}_{2}$ is typically unavailable in such a setup. However, we will not dig any deeper into this subject here. Instead it is assumed that an estimator deploying ICIP has access to all information necessary for using ICIP.

Several examples of fusion using ICIP are provided in Example 4.1-4.3. CI and the naïve estimator are compared to ICIP. The BLUE has been left out since it depends on the actual cross-correlation and we only want to compare how the covariance matrices differ in relation to what is assumed about the crosscorrelations. The covariances $\mathbf{P}^{\mathrm{CI}}$ and $\mathbf{P}^{\mathrm{ICIP}}$ are found by optimizing on $\operatorname{tr}\left(\mathbf{P}^{\mathrm{CI}}\right)$ and $\operatorname{tr}\left(\mathbf{P}^{\mathrm{ICIP}}\right)$, respectively.

\section{Example 4.1: Inverse Covariance Intersection Partial Estimate Form}

Assume that we have

$$
\begin{aligned}
& \mathbf{C}_{1}=\left[\begin{array}{ll}
4 & 0 \\
0 & 1
\end{array}\right], \quad \mathbf{H}_{1}=\left[\begin{array}{ll}
1 & 0 \\
0 & 1
\end{array}\right], \quad \boldsymbol{\Gamma}_{1}=4, \quad \mathbf{G}_{1}=\left[\begin{array}{ll}
1 & 0
\end{array}\right], \\
& \mathbf{C}_{2}=2, \quad \mathbf{H}_{2}=\left[\begin{array}{ll}
1 & 0
\end{array}\right], \quad \boldsymbol{\Gamma}_{2}=4, \quad \mathbf{G}_{2}=1 \text {. }
\end{aligned}
$$


If $\alpha=1$

$$
\begin{gathered}
\mathbf{H}_{1} \mathbf{H}_{2}^{\top} \mathbf{C}_{2}^{-1} \mathbf{H}_{2} \mathbf{H}_{1}^{\top}=\left[\begin{array}{cc}
\frac{1}{4} & 0 \\
0 & 0
\end{array}\right] \geq \alpha\left[\begin{array}{cc}
\frac{1}{4} & 0 \\
0 & 0
\end{array}\right]=\alpha \mathbf{G}_{1}^{\top} \boldsymbol{\Gamma}_{1}^{-1} \mathbf{G}_{1} \boldsymbol{\Gamma}_{1} \mathbf{G}_{1}^{\top} \boldsymbol{\Gamma}_{1}^{-1} \mathbf{G}_{1}, \\
\mathbf{H}_{2} \mathbf{H}_{1}^{\top} \mathbf{C}_{1}^{-1} \mathbf{H}_{1} \mathbf{H}_{2}^{\top}=\frac{1}{2} \geq \frac{1}{\alpha} \frac{1}{4}=\frac{1}{\alpha} \mathbf{G}_{2}^{\top} \boldsymbol{\Gamma}_{2}^{-1} \mathbf{G}_{2} \boldsymbol{\Gamma}_{2} \mathbf{G}_{2}^{\top} \boldsymbol{\Gamma}_{2}^{-1} \mathbf{G}_{2},
\end{gathered}
$$

is satisfied, meaning ICIP will provide a conservatively fused estimate in this case (see Theorem 4.5). The covariance matrices resulting from fusion of $\mathbf{C}_{1}$ and $\mathbf{C}_{2}$ using CI, ICIP and the naïve fusion rule are respectively given by

$$
\mathbf{P}^{\mathrm{CI}}=\left[\begin{array}{cc}
3 & 0 \\
0 & \frac{3}{2}
\end{array}\right], \quad \mathbf{P}^{\mathrm{ICIP}}=\left[\begin{array}{ll}
2 & 0 \\
0 & 1
\end{array}\right], \quad \mathbf{P}^{\mathrm{N}}=\left[\begin{array}{ll}
\frac{4}{3} & 0 \\
0 & 1
\end{array}\right] .
$$

Hence $\mathbf{P}^{\mathrm{CI}}>\mathbf{P}^{\mathrm{ICIP}} \geq \mathbf{P}^{\mathrm{N}}$. Both $\mathbf{P}^{\mathrm{CI}}$ and $\mathbf{P}^{\mathrm{ICIP}}$ are optimized on the trace. Note, $\left[\mathbf{P}^{\mathrm{CI}}\right]_{22}>\left[\mathbf{C}_{1}\right]_{22}$ while $\operatorname{tr}\left(\mathbf{C}_{1}\right)>\operatorname{tr}\left(\mathbf{P}^{\mathrm{CI}}\right)$.

\section{Example 4.2: Inverse Covariance Intersection Partial Estimate Form}

Assume that we have

$$
\begin{array}{llll}
\mathbf{C}_{1}=\left[\begin{array}{ll}
1 & 0 \\
0 & 2
\end{array}\right], & \mathbf{H}_{1}=\left[\begin{array}{lll}
1 & 0 & 0 \\
0 & 1 & 0
\end{array}\right], & \boldsymbol{\Gamma}_{1}=4, & \mathbf{G}_{1}=\left[\begin{array}{ll}
0 & 1
\end{array}\right], \\
\mathbf{C}_{2}=\left[\begin{array}{ll}
2 & 0 \\
0 & 1
\end{array}\right], & \mathbf{H}_{2}=\left[\begin{array}{lll}
0 & 1 & 0 \\
0 & 0 & 1
\end{array}\right], & \boldsymbol{\Gamma}_{2}=4, & \mathbf{G}_{2}=\left[\begin{array}{ll}
1 & 0
\end{array}\right],
\end{array}
$$

such that $\operatorname{rank}\left(\left[\begin{array}{ll}\mathbf{H}_{1}^{\top} & \mathbf{H}_{2}^{\top}\end{array}\right]^{\top}\right)=n_{x}=3$. Hence we get

$$
\begin{aligned}
& \mathbf{H}_{1} \mathbf{H}_{2}^{\top} \mathbf{C}_{2}^{-1} \mathbf{H}_{2} \mathbf{H}_{1}^{\top}=\left[\begin{array}{ll}
0 & 0 \\
0 & \frac{1}{2}
\end{array}\right] \geq \alpha\left[\begin{array}{cc}
0 & 0 \\
0 & \frac{1}{4}
\end{array}\right]=\alpha \mathbf{G}_{1}^{\top} \boldsymbol{\Gamma}_{1}^{-1} \mathbf{G}_{1} \boldsymbol{\Gamma}_{1} \mathbf{G}_{1}^{\top} \boldsymbol{\Gamma}_{1}^{-1} \mathbf{G}_{1}, \\
& \mathbf{H}_{2} \mathbf{H}_{1}^{\top} \mathbf{C}_{1}^{-1} \mathbf{H}_{1} \mathbf{H}_{2}^{\top}=\left[\begin{array}{cc}
\frac{1}{2} & 0 \\
0 & 0
\end{array}\right] \geq \frac{1}{\alpha}\left[\begin{array}{cc}
\frac{1}{4} & 0 \\
0 & 0
\end{array}\right]=\frac{1}{\alpha} \mathbf{G}_{2}^{\top} \boldsymbol{\Gamma}_{2}^{-1} \mathbf{G}_{2} \boldsymbol{\Gamma}_{2} \mathbf{G}_{2}^{\top} \boldsymbol{\Gamma}_{2}^{-1} \mathbf{G}_{2},
\end{aligned}
$$

which will be satisfied if $\alpha=1$. The covariances resulting from fusion of $\mathbf{C}_{1}$ and $\mathrm{C}_{2}$ using CI, ICIP and the naïve fusion rule are respectively given by

$$
\mathbf{P}^{\mathrm{CI}}=\left[\begin{array}{lll}
2 & 0 & 0 \\
0 & 2 & 0 \\
0 & 0 & 2
\end{array}\right], \quad \mathbf{P}^{\mathrm{ICIP}}=\left[\begin{array}{lll}
1 & 0 & 0 \\
0 & 2 & 0 \\
0 & 0 & 1
\end{array}\right], \quad \mathbf{P}^{\mathrm{N}}=\left[\begin{array}{lll}
1 & 0 & 0 \\
0 & 1 & 0 \\
0 & 0 & 1
\end{array}\right],
$$

i.e., $\mathbf{P}^{\mathrm{CI}} \geq \mathbf{P}^{\mathrm{ICIP}} \geq \mathbf{P}^{\mathrm{N}}$ in this case. Both $\mathbf{P}^{\mathrm{CI}}$ and $\mathbf{P}^{\mathrm{ICIP}}$ are optimized on the trace. Note, neither of the input estimates is a full estimate. Therefore a full estimate must use components from both input estimates. Otherwise the trace of the covariance of the full estimate goes to infinity, e.g., if $\omega \in\{0,1\}$ then $\operatorname{tr}\left(\mathbf{P}^{\mathrm{CI}}(\omega)\right) \rightarrow \infty$. 


\section{Example 4.3: Inverse Covariance Intersection Partial Estimate Form}

Assume that we have
$\mathbf{C}_{1}=\left[\begin{array}{ll}1 & 0 \\ 0 & 2\end{array}\right]$,
$\mathbf{H}_{1}=\left[\begin{array}{lll}1 & 0 & 0 \\ 0 & 1 & 0\end{array}\right]$,
$\Gamma_{1}=\left[\begin{array}{ll}4 & 0 \\ 0 & 4\end{array}\right]$
$\mathbf{G}_{1}=\left[\begin{array}{ll}1 & 0 \\ 0 & 1\end{array}\right]$
$\mathbf{C}_{2}=\left[\begin{array}{ll}2 & 0 \\ 0 & 1\end{array}\right]$,
$\mathbf{H}_{2}=\left[\begin{array}{lll}0 & 1 & 0 \\ 0 & 0 & 1\end{array}\right]$,
$\Gamma_{2}=4$,
$\mathbf{G}_{2}=\left[\begin{array}{ll}1 & 0\end{array}\right]$.

In this case

$$
\mathbf{H}_{1} \mathbf{H}_{2}^{\top} \mathbf{C}_{2}^{-1} \mathbf{H}_{2} \mathbf{H}_{1}^{\top}=\left[\begin{array}{cc}
0 & 0 \\
0 & \frac{1}{2}
\end{array}\right] \geq \alpha\left[\begin{array}{cc}
\frac{1}{4} & 0 \\
0 & \frac{1}{4}
\end{array}\right]=\alpha \mathbf{G}_{1}^{\top} \boldsymbol{\Gamma}_{1}^{-1} \mathbf{G}_{1} \boldsymbol{\Gamma}_{1} \mathbf{G}_{1}^{\top} \boldsymbol{\Gamma}_{1}^{-1} \mathbf{G}_{1},
$$

will not be satisfied for any $\alpha>0$. Hence, ICIP cannot be guaranteed to provide a conservative estimate.

\section{Properties}

Theorem 4.3 states that the ICIP method can be expressed on the WLS estimator form. In Theorem 4.4 it is shown that ICIP reduces to ICI if $\mathbf{H}_{1}=\mathbf{H}_{2}=\mathbf{I}$ and $\mathbf{G}_{1}=\mathbf{G}_{2}=\mathbf{I}$.

Theorem 4.3 (Inverse Covariance Intersection-Weighted Least Squares). The inverse covariance intersection estimator as given in Algorithm 4.2 can be expressed as a weighted least squares estimator, with joint covariance

$$
\mathbf{C}_{J}^{\mathrm{ICIP}}=\left[\begin{array}{cc}
\mathbf{C}_{1}+\frac{\omega}{1-\omega} \mathbf{C}_{1} \mathbf{H}_{1} \mathbf{H}_{2}^{\top} \mathbf{C}_{2}^{-1} \mathbf{H}_{2} \mathbf{H}_{1}^{\top} \mathbf{C}_{1} & \mathbf{0} \\
\mathbf{0} & \mathbf{C}_{2}+\frac{1-\omega}{\omega} \mathbf{C}_{2} \mathbf{H}_{2} \mathbf{H}_{1}^{\top} \mathbf{C}_{1}^{-1} \mathbf{H}_{1} \mathbf{H}_{2}^{\top} \mathbf{C}_{2}
\end{array}\right],
$$

and modified weight matrix $\left(\mathbf{C}_{J}^{\mathrm{ICIP}}\right)^{-1}$, where $\omega \in[0,1]$.

Proof: Let $\hat{\mathbf{z}}_{1}=\mathbf{H}_{1} \mathbf{x}+\mathbf{e}_{1}$ and $\hat{\mathbf{z}}_{2}=\mathbf{H}_{2} \mathbf{x}+\mathbf{e}_{2}$. Let $\hat{\mathbf{z}}_{J}=\operatorname{col}\left(\hat{\mathbf{z}}_{1}, \hat{\mathbf{z}}_{2}\right)$ and $\mathbf{H}_{J}=$ $\operatorname{col}\left(\mathbf{H}_{1}, \mathbf{H}_{2}\right)$. Assume the joint covariance is given by

$$
\mathbf{C}_{J}^{\mathrm{ICIP}}=\left[\begin{array}{cc}
\mathbf{C}_{1}+\frac{\omega}{1-\omega} \mathbf{C}_{1} \mathbf{H}_{1} \mathbf{H}_{2}^{\top} \mathbf{C}_{2}^{-1} \mathbf{H}_{2} \mathbf{H}_{1}^{\top} \mathbf{C}_{1} & \mathbf{0} \\
\mathbf{0} & \mathbf{C}_{2}+\frac{1-\omega}{\omega} \mathbf{C}_{2} \mathbf{H}_{2} \mathbf{H}_{1}^{\top} \mathbf{C}_{1}^{-1} \mathbf{H}_{1} \mathbf{H}_{2}^{\top} \mathbf{C}_{2}
\end{array}\right] .
$$

As in (4.44) the covariance of the fused estimate can be calculated using the WLS formula of (3.35) as

$$
\begin{aligned}
\mathbf{P}^{-1}= & \mathbf{H}_{J}^{\top}\left(\mathbf{C}_{J}^{\mathrm{ICIP}}\right)^{-1} \mathbf{H}_{J} \\
= & \mathbf{H}_{1}^{\top} \mathbf{C}_{1}^{-1} \mathbf{H}_{1}-\mathbf{H}_{1}^{\top} \mathbf{H}_{1} \mathbf{H}_{2}^{\top}\left(\frac{1-\omega}{\omega} \mathbf{C}_{2}+\mathbf{H}_{2} \mathbf{H}_{1}^{\top} \mathbf{C}_{1} \mathbf{H}_{1} \mathbf{H}_{2}^{\top}\right)^{-1} \mathbf{H}_{2} \mathbf{H}_{1}^{\top} \mathbf{H}_{1} \\
& +\mathbf{H}_{2}^{\top} \mathbf{C}_{2}^{-1} \mathbf{H}_{2}-\mathbf{H}_{2}^{\top} \mathbf{H}_{2} \mathbf{H}_{1}^{\top}\left(\frac{\omega}{1-\omega} \mathbf{C}_{1}+\mathbf{H}_{1} \mathbf{H}_{2}^{\top} \mathbf{C}_{2} \mathbf{H}_{2} \mathbf{H}_{1}^{\top}\right)^{-1} \mathbf{H}_{1} \mathbf{H}_{2}^{\top} \mathbf{H}_{2} \\
= & \mathbf{H}_{1}^{\top} \mathbf{C}_{1}^{-1} \mathbf{H}_{1}-\omega \mathbf{H}_{1}^{\top} \mathbf{H}_{1} \mathbf{H}_{2}^{\top}\left(\omega \mathbf{H}_{2} \mathbf{H}_{1}^{\top} \mathbf{C}_{1} \mathbf{H}_{1} \mathbf{H}_{2}^{\top}+(1-\omega) \mathbf{C}_{2}\right)^{-1} \mathbf{H}_{2} \mathbf{H}_{1}^{\top} \mathbf{H}_{1} \\
& +\mathbf{H}_{2}^{\top} \mathbf{C}_{2}^{-1} \mathbf{H}_{2}-(1-\omega) \mathbf{H}_{2}^{\top} \mathbf{H}_{2} \mathbf{H}_{1}^{\top}\left(\omega \mathbf{C}_{1}+(1-\omega) \mathbf{H}_{1} \mathbf{H}_{2}^{\top} \mathbf{C}_{2} \mathbf{H}_{2} \mathbf{H}_{1}^{\top}\right)^{-1} \mathbf{H}_{1} \mathbf{H}_{2}^{\top} \mathbf{H}_{2} .
\end{aligned}
$$


Similarly, the estimate can be expressed in information form as

$$
\begin{aligned}
\mathbf{P}^{-1} \hat{\mathbf{x}} & \\
= & \mathbf{H}_{J}^{\top}\left(\mathbf{C}_{J}^{\mathrm{ICIP}}\right)^{-1} \hat{\mathbf{z}}_{J} \\
= & \left(\mathbf{H}_{1}^{\top} \mathbf{C}_{1}^{-1}-\omega \mathbf{H}_{1}^{\top} \mathbf{H}_{1} \mathbf{H}_{2}^{\top}\left(\omega \mathbf{H}_{2} \mathbf{H}_{1}^{\top} \mathbf{C}_{1} \mathbf{H}_{1} \mathbf{H}_{2}^{\top}+(1-\omega) \mathbf{C}_{2}\right)^{-1} \mathbf{H}_{2} \mathbf{H}_{1}^{\top}\right) \hat{\mathbf{z}}_{1} \\
& +\left(\mathbf{H}_{2}^{\top} \mathbf{C}_{2}^{-1}-(1-\omega) \mathbf{H}_{2}^{\top} \mathbf{H}_{2} \mathbf{H}_{1}^{\top}\left(\omega \mathbf{C}_{1}+(1-\omega) \mathbf{H}_{1} \mathbf{H}_{2}^{\top} \mathbf{C}_{2} \mathbf{H}_{2} \mathbf{H}_{1}^{\top}\right)^{-1} \mathbf{H}_{1} \mathbf{H}_{2}^{\top}\right) \hat{\mathbf{z}}_{2},
\end{aligned}
$$

which is equivalent to the ICIP method of Algorithm 4.2. Hence, ICIP can be expressed on WLS form with joint covariance $C_{J}^{I C I P}$ and weight matrix $\left(\mathbf{C}_{J}^{\mathrm{ICIP}}\right)^{-1}$.

Corollary 4.2 (Inverse Covariance Intersection-Linear Unbiased). The inverse covariance intersection algorithm as given in Algorithm 4.2 is a linear unbiased estimator.

Proof: Since the WLS estimator is linear unbiased it follows directly from Theorem 4.3 that also the ICIP algorithm is a linear unbiased estimator, given that the input estimates are unbiased.

Theorem 4.4 (Inverse Covariance Intersection-Special Case). If $\mathbf{H}_{1}=\mathbf{H}_{2}=$ $\mathbf{G}_{1}=\mathbf{G}_{2}=\mathbf{I}$ the partial estimate form of inverse covariance intersection as given in Algorithm 4.2 reduces to the inverse covariance intersection algorithm of (4.35).

Proof: It will be shown that if $\mathbf{H}_{1}=\mathbf{H}_{2}=\mathbf{G}_{1}=\mathbf{G}_{2}=\mathbf{I}$ then

1. The decomposition in (4.41) reduces to (4.37),

2. The conditions in (4.47) reduces to (4.39), and

3. The partial estimate form of inverse covariance intersection as given in Algorithm 4.2 reduces to the formulas given in (4.35).

Since $\Gamma_{l}^{-1}=\Gamma_{\imath}^{-1} \Gamma_{\imath} \Gamma_{l}^{-1}$ the statements in 1 . and 2. follow immediately by adopting $\mathbf{H}_{1}=\mathbf{H}_{2}=\mathbf{G}_{1}=\mathbf{G}_{2}=\mathbf{I}$ in (4.41) and (4.47), respectively. Statement 3 . holds since $\mathbf{H}_{1}=\mathbf{H}_{2}=\mathbf{I}$ implies (4.45a) which simplifies into

$$
\begin{aligned}
\mathbf{P}^{-1} & =\mathbf{C}_{1}^{-1}-\omega\left(\omega \mathbf{C}_{1}+(1-\omega) \mathbf{C}_{2}\right)^{-1}+\mathbf{C}_{2}^{-1}-(1-\omega)\left(\omega \mathbf{C}_{1}+(1-\omega) \mathbf{C}_{2}\right)^{-1} \\
& =\mathbf{C}_{1}^{-1}+\mathbf{C}_{2}^{-1}-(1-\omega+\omega)\left(\omega \mathbf{C}_{1}+(1-\omega) \mathbf{C}_{2}\right)^{-1} \\
& =\mathbf{C}_{1}^{-1}+\mathbf{C}_{2}^{-1}-\left(\omega \mathbf{C}_{1}+(1-\omega) \mathbf{C}_{2}\right)^{-1}
\end{aligned}
$$

and similarly for $(4.45 \mathrm{~b})$

$$
\begin{aligned}
\mathbf{P}^{-1} \hat{\mathbf{x}}= & \left(\mathbf{C}_{1}^{-1}-\omega\left(\omega \mathbf{C}_{1}+(1-\omega) \mathbf{C}_{2}\right)^{-1}\right) \hat{\mathbf{z}}_{1} \\
& +\left(\mathbf{C}_{2}^{-1}-(1-\omega)\left(\omega \mathbf{C}_{1}+(1-\omega) \mathbf{C}_{2}\right)^{-1}\right) \hat{\mathbf{z}}_{2} .
\end{aligned}
$$


Theorem 4.5 states under which terms conservativeness of ICIP can be guaranteed. The proof is similar to the corresponding proof given in [48]. Optimality of ICI is proven in Theorem 4.6.

Theorem 4.5 (Inverse Covariance Intersection-Conservative). The inverse covariance intersection method, defined in Algorithm 4.2, for fusion of the partial estimates $\hat{\mathbf{z}}_{1}=\mathbf{H}_{1} \mathbf{x}+\mathbf{e}_{1}$ and $\hat{\mathbf{z}}_{2}=\mathbf{H}_{2} \mathbf{x}+\mathbf{e}_{2}$, where $\mathbf{C}_{1} \geq \operatorname{cov}\left(\hat{\mathbf{z}}_{1}\right)$ and $\mathbf{C}_{2} \geq \operatorname{cov}\left(\hat{\mathbf{z}}_{2}\right)$, produces conservative estimates if the following is true:

- The estimates can be decomposed according to (4.41).

- The conditions in (4.47) are satisfied.

Proof: According to Theorem 3.2 it is sufficient to show $\mathbf{C}_{J}^{\mathrm{ICIP}} \geq \mathbf{C}_{J}$. From (4.43) and by letting $\mu=\frac{1-\omega}{\omega}$

$$
\begin{aligned}
\tilde{\mathbf{C}}_{J} & =\mathbf{C}_{J}^{\text {ICIP }}-\mathbf{C}_{J} \\
& =\left[\begin{array}{cc}
\frac{1}{\mu} \mathbf{C}_{1} \mathbf{H}_{1} \mathbf{H}_{2}^{\top} \mathbf{C}_{2}^{-1} \mathbf{H}_{2} \mathbf{H}_{1}^{\top} \mathbf{C}_{1} & -\mathbf{C}_{12} \\
-\mathbf{C}_{21} & \mu \mathbf{C}_{2} \mathbf{H}_{2} \mathbf{H}_{1}^{\top} \mathbf{C}_{1}^{-1} \mathbf{H}_{1} \mathbf{H}_{2}^{\top} \mathbf{C}_{2}
\end{array}\right] \\
& =\left[\begin{array}{cc}
\frac{1}{\mu} \mathbf{C}_{1} \mathbf{H}_{1} \mathbf{H}_{2}^{\top} \mathbf{C}_{2}^{-1} \mathbf{H}_{2} \mathbf{H}_{1}^{\top} \mathbf{C}_{1} & -\mathbf{C}_{1} \mathbf{G}_{1}^{\top} \boldsymbol{\Gamma}_{1}^{-1} \mathbf{G}_{1} \boldsymbol{\Gamma}_{12} \mathbf{G}_{2}^{\top} \boldsymbol{\Gamma}_{2}^{-1} \mathbf{G}_{2} \mathbf{C}_{2} \\
-\mathbf{C}_{2} \mathbf{G}_{2}^{\top} \boldsymbol{\Gamma}_{2}^{-1} \mathbf{G}_{2} \boldsymbol{\Gamma}_{21} \mathbf{G}_{1}^{\top} \boldsymbol{\Gamma}_{1}^{-1} \mathbf{G}_{1} \mathbf{C}_{1} & \mu \mathbf{C}_{2} \mathbf{H}_{2} \mathbf{H}_{1}^{\top} \mathbf{C}_{1}^{-1} \mathbf{H}_{1} \mathbf{H}_{2}^{\top} \mathbf{C}_{2}
\end{array}\right] \\
& \geq\left[\begin{array}{cc}
\frac{\alpha}{\mu} \mathbf{C}_{1} \mathbf{G}_{1}^{\top} \boldsymbol{\Gamma}_{1}^{-1} \mathbf{G}_{1} \boldsymbol{\Gamma}_{1} \mathbf{G}_{1}^{\top} \boldsymbol{\Gamma}_{1}^{-1} \mathbf{G}_{1} \mathbf{C}_{1} & -\mathbf{C}_{1} \mathbf{G}_{1}^{\top} \boldsymbol{\Gamma}_{1}^{-1} \mathbf{G}_{1} \boldsymbol{\Gamma}_{12} \mathbf{G}_{2}^{\top} \boldsymbol{\Gamma}_{2}^{-1} \mathbf{G}_{2} \mathbf{C}_{2} \\
-\mathbf{C}_{2} \mathbf{G}_{2}^{\top} \mathbf{\Gamma}_{2}^{-1} \mathbf{G}_{2} \boldsymbol{\Gamma}_{21} \mathbf{G}_{1}^{\top} \boldsymbol{\Gamma}_{1}^{-1} \mathbf{G}_{1} \mathbf{C}_{1} & \frac{\mu}{\alpha} \mathbf{C}_{2} \mathbf{G}_{2}^{\top} \mathbf{\Gamma}_{2}^{-1} \mathbf{G}_{2} \boldsymbol{\Gamma}_{2} \mathbf{G}_{2}^{\top} \boldsymbol{\Gamma}_{2}^{-1} \mathbf{G}_{2} \mathbf{C}_{2}
\end{array}\right],
\end{aligned}
$$

where in the second last step and in the last step (4.42) and (4.47) were used, respectively. $\tilde{\mathbf{C}}_{J}$ will be positive semi-definite if

$$
\left[\begin{array}{l}
\mathbf{y}_{1} \\
\mathbf{y}_{2}
\end{array}\right]^{\top} \tilde{\mathbf{C}}_{J}\left[\begin{array}{l}
\mathbf{y}_{1} \\
\mathbf{y}_{2}
\end{array}\right] \geq \mathbf{0}
$$

holds for all $\mathrm{y}_{1}$ and $\mathrm{y}_{2}$. After the coordinate transformation

$$
\mathbf{y}_{1}^{\prime}=\sqrt{\frac{\alpha}{\mu}} \mathbf{G}_{1}^{\top} \boldsymbol{\Gamma}_{1}^{-1} \mathbf{G}_{1} \mathbf{C}_{1} \mathbf{y}_{1}, \quad \mathbf{y}_{2}^{\prime}=-\sqrt{\frac{\mu}{\alpha}} \mathbf{G}_{2}^{\top} \boldsymbol{\Gamma}_{2}^{-1} \mathbf{G}_{2} \mathbf{C}_{2} \mathbf{y}_{2},
$$

the quadratic form above turns into

$$
\left[\begin{array}{l}
y_{1}^{\prime} \\
y_{2}^{\prime}
\end{array}\right]^{\top}\left[\begin{array}{cc}
\Gamma_{1} & \Gamma_{12} \\
\Gamma_{21} & \Gamma_{2}
\end{array}\right]\left[\begin{array}{l}
y_{1}^{\prime} \\
y_{2}^{\prime}
\end{array}\right]
$$

which is nonzero for all $y_{1}^{\prime}$ and $y_{2}^{\prime}$ under the assumption made in (4.40).

Theorem 4.6 (Inverse Covariance Intersection-Optimal). Assume $\hat{\mathbf{z}}_{1}=\mathbf{x}+\mathbf{e}_{1}$ and $\hat{\mathbf{z}}_{2}=\mathbf{x}+\mathbf{e}_{2}$ are given, where $\mathbf{C}_{1} \geq \operatorname{cov}\left(\hat{\mathbf{z}}_{1}\right)$ and $\mathbf{C}_{2} \geq \operatorname{cov}\left(\hat{\mathbf{z}}_{2}\right)$. If the correlated information decompositions of (4.37) hold and the conditions of (4.39) are satisfied, then the inverse covariance intersection algorithm as given in (4.35) solves the problem in (4.33) and hence is a restricted best conservative linear unbiased estimator. 
Proof: From Theorem 4.4 and Theorem 4.5 it follows that ICI is conservative under the assumed conditions since $\mathbf{C}_{J}^{\mathrm{ICI}} \geq \mathbf{C}_{J}$, where

$$
\mathbf{C}_{J}^{\mathrm{ICI}}=\left[\begin{array}{cc}
\mathbf{C}_{1}+\frac{\omega}{1-\omega} \mathbf{C}_{1} \mathbf{C}_{2}^{-2} \mathbf{C}_{1} & 0 \\
0 & \mathbf{C}_{2}+\frac{1-\omega}{\omega} \mathbf{C}_{2} \mathbf{C}_{1}^{-2} \mathbf{C}_{2}
\end{array}\right], \quad \mathbf{C}_{J}=\left[\begin{array}{cc}
\mathbf{C}_{1} & \mathbf{C}_{12} \\
\mathbf{C}_{21} & \mathbf{C}_{2}
\end{array}\right] .
$$

The following will be shown

$$
\mathrm{C}_{J}^{\mathrm{ICI}} \geq \overline{\mathbf{C}}_{J} \geq \mathrm{C}_{J} \Longrightarrow \overline{\mathbf{C}}_{J}=\mathrm{C}_{J}^{\mathrm{ICI}},
$$

where $\overline{\mathbf{C}}_{J}=\operatorname{diag}\left(\overline{\mathbf{C}}_{1}, \overline{\mathbf{C}}_{2}\right)$, from which this theorem follows by application of Theorem 3.2.

By assumption

$$
\begin{aligned}
& \mathbf{C}_{1}+\frac{\omega}{1-\omega} \mathbf{C}_{1} \mathbf{C}_{2}^{-1} \mathbf{C}_{1} \geq \overline{\mathbf{C}}_{1} \geq \mathbf{C}_{1} \Longrightarrow\left(\overline{\mathbf{C}}_{1}-\mathbf{C}_{1}\right)^{-1} \geq\left(\frac{\omega}{1-\omega} \mathbf{C}_{1} \mathbf{C}_{2}^{-1} \mathbf{C}_{1}\right)^{-1}, \\
& \mathbf{C}_{2}+\frac{1-\omega}{\omega} \mathbf{C}_{2} \mathbf{C}_{1}^{-1} \mathbf{C}_{2} \geq \overline{\mathbf{C}}_{2} \geq \mathbf{C}_{2} \Longrightarrow\left(\overline{\mathbf{C}}_{2}-\mathbf{C}_{2}\right)^{-1} \geq\left(\frac{1-\omega}{\omega} \mathbf{C}_{2} \mathbf{C}_{1}^{-1} \mathbf{C}_{2}\right)^{-1}
\end{aligned}
$$

The conditions

$$
\mathbf{C}_{1}^{-1} \geq \frac{1}{\alpha} \boldsymbol{\Gamma}_{2}^{-2}, \quad \mathbf{C}_{2}^{-1} \geq \alpha \boldsymbol{\Gamma}_{1}^{-1}, \quad \boldsymbol{\Gamma}_{1} \geq \boldsymbol{\Gamma}_{12} \boldsymbol{\Gamma}_{2}^{-1} \boldsymbol{\Gamma}_{21}, \quad \boldsymbol{\Gamma}_{2} \geq \boldsymbol{\Gamma}_{21} \boldsymbol{\Gamma}_{1}^{-1} \boldsymbol{\Gamma}_{12} .
$$

must be obeyed also by $\overline{\mathbf{C}}_{J}$. $\overline{\mathbf{C}}_{J} \geq \mathbf{C}_{J}$ is true if and only if [19]

$$
\begin{aligned}
& \overline{\mathbf{C}}_{1}-\mathbf{C}_{1} \geq \mathbf{C}_{12}\left(\overline{\mathbf{C}}_{2}-\mathbf{C}_{2}\right)^{-1} \mathbf{C}_{21} \geq \mathbf{C}_{12}\left(\frac{1-\omega}{\omega} \mathbf{C}_{2} \mathbf{C}_{1}^{-1} \mathbf{C}_{2}\right)^{-1} \mathbf{C}_{21}, \\
& \overline{\mathbf{C}}_{2}-\mathbf{C}_{2} \geq \mathbf{C}_{21}\left(\overline{\mathbf{C}}_{1}-\mathbf{C}_{1}\right)^{-1} \mathbf{C}_{12} \geq \mathbf{C}_{21}\left(\frac{\omega}{1-\omega} \mathbf{C}_{1} \mathbf{C}_{2}^{-1} \mathbf{C}_{1}\right)^{-1} \mathbf{C}_{12},
\end{aligned}
$$

where (4.49) has been used in the last step of each row. From the conditions in (4.50) and using $\mathbf{C}_{12}=\mathbf{C}_{1} \Gamma_{1}^{-1} \Gamma_{12} \Gamma_{2}^{-1} \mathbf{C}_{2}$ it follows that $\overline{\mathbf{C}}_{1}$ must also satisfy

$$
\begin{aligned}
& \overline{\mathbf{C}}_{1}-\mathbf{C}_{1}-\mathbf{C}_{12} \frac{\omega}{1-\omega} \mathbf{C}_{2}^{-1} \mathbf{C}_{1} \mathbf{C}_{2}^{-1} \mathbf{C}_{21} \\
& =\overline{\mathbf{C}}_{1}-\mathbf{C}_{1}-\frac{\omega}{1-\omega} \mathbf{C}_{1} \boldsymbol{\Gamma}_{1}^{-1} \boldsymbol{\Gamma}_{12} \boldsymbol{\Gamma}_{2}^{-1} \mathbf{C}_{2} \mathbf{C}_{2}^{-1} \mathbf{C}_{1} \mathbf{C}_{2}^{-1} \mathbf{C}_{2} \boldsymbol{\Gamma}_{2}^{-1} \boldsymbol{\Gamma}_{21} \boldsymbol{\Gamma}_{1}^{-1} \mathbf{C}_{1} \\
& \geq \overline{\mathbf{C}}_{1}-\mathbf{C}_{1}-\frac{\omega}{1-\omega} \mathbf{C}_{1} \boldsymbol{\Gamma}_{1}^{-1} \boldsymbol{\Gamma}_{12} \Gamma_{2}^{-1} \alpha \boldsymbol{\Gamma}_{2} \Gamma_{2}^{-1} \Gamma_{21} \Gamma_{1}^{-1} \mathbf{C}_{1} \\
& \geq \overline{\mathbf{C}}_{1}-\mathbf{C}_{1}-\frac{\omega}{1-\omega} \mathbf{C}_{1} \Gamma_{1}^{-1} \alpha \boldsymbol{\Gamma}_{1} \Gamma_{1}^{-1} \mathbf{C}_{1} \\
& \geq \overline{\mathbf{C}}_{1}-\mathbf{C}_{1}-\frac{\omega}{1-\omega} \mathbf{C}_{1} \alpha \Gamma_{1}^{-1} \mathbf{C}_{1} \\
& \geq \overline{\mathbf{C}}_{1}-\mathbf{C}_{1}-\frac{\omega}{1-\omega} \mathbf{C}_{1} \mathbf{C}_{2}^{-1} \mathbf{C}_{1} \\
& \geq \mathbf{0}
\end{aligned}
$$


which yields $\overline{\mathbf{C}}_{1} \geq \mathbf{C}_{1}+\frac{\omega}{1-\omega} \mathbf{C}_{1} \mathbf{C}_{2}^{-2} \mathbf{C}_{1}$. By assumption $\overline{\mathbf{C}}_{1} \leq \mathbf{C}_{1}+\frac{\omega}{1-\omega} \mathbf{C}_{1} \mathbf{C}_{2}^{-2} \mathbf{C}_{1}$, which is only possible if $\overline{\mathbf{C}}_{1}=\mathbf{C}_{1}+\frac{\omega}{1-\omega} \mathbf{C}_{1} \mathbf{C}_{2}^{-2} \mathbf{C}_{1}$. Corresponding calculations for $\overline{\mathbf{C}}_{2}$ yield $\overline{\mathbf{C}}_{2}=\mathbf{C}_{2}+\frac{1-\omega}{\omega} \mathbf{C}_{2} \mathbf{C}_{1}^{-1} \mathbf{C}_{2}$.

We have shown that under the assumed decompositions in (4.39) and the conditions in (4.50) $\mathrm{C}_{J}^{\mathrm{ICI}}$ is the МСB. Hence, using Theorem 3.2 it is concluded that ICI gives an optimal solution (a restricted best CLUE) to (4.33) under the given assumptions.

\subsection{Fusion Under Component-Wise Aligned Correlations}

The component-wise aligned structure is defined, after which the LE method is presented and analyzed.

\subsubsection{Component-Wise Aligned Correlations}

The component-wise aligned structure might arise in configurations with common process noise where, e.g., the process noise covariance $\mathbf{Q}$ is diagonal while the measurement update does not break component-wise aligned structure. In practice it is however probably more interesting to see when the component-wise structure is approximately satisfied since some coupling between different components will be present in more or less all scenarios.

Cross-correlations are called component-wise aligned if there exists a joint transformation matrix

$$
\mathbf{T}_{J}=\left[\begin{array}{cc}
\mathbf{T} & \mathbf{0} \\
\mathbf{0} & \mathbf{T}
\end{array}\right],
$$

such that

$$
\mathbf{D}_{J}=\mathbf{T}_{J} \mathbf{C}_{J} \mathbf{T}_{J}^{\top}=\left[\begin{array}{cc}
\mathbf{D}_{1} & \mathbf{D}_{12} \\
\mathbf{D}_{21} & \mathbf{D}_{2}
\end{array}\right],
$$

where $\mathbf{D}_{1}, \mathbf{D}_{2}$ and $\mathbf{D}_{12}=\mathbf{D}_{21}$ are diagonal.

\subsubsection{Largest Ellipsoid Method}

Yet another less conservative alternative to CI, but also to ICI, is the largest ellipsoid (LE) method proposed in 2002 by Benaskeur [5]. The LE method has come with different names, in [18] it is called safe fusion (SF), in [59,60] it is named ellipsoidal intersection (EI), and [69] suggests the name internal ellipsoid approximation (IEA). Minor differences between the above mentioned methods exist. However, these differences are not investigated here. LE is related to the component-wise aligned structure. 


\section{Algorithm 4.3: Largest Ellipsoid Method}

Input: $\hat{\mathbf{z}}_{1}, \hat{\mathbf{z}}_{2}, \mathbf{C}_{1}, \mathbf{C}_{2}$

The estimates $\hat{\mathbf{z}}_{1}$ and $\hat{\mathbf{z}}_{2}$ are fused according to:

1. Decompose $\mathbf{C}_{1}=\mathbf{U}_{1} \mathbf{D}_{1} \mathbf{U}_{1}^{\top}$ and let $\mathbf{T}_{1}=\mathbf{D}_{1}^{-\frac{1}{2}} \mathbf{U}_{1}^{\top}$. Decompose $\mathbf{T}_{1} \mathbf{C}_{2} \mathbf{T}_{1}^{\top}=$ $\mathbf{U}_{2} \mathbf{D}_{2} \mathbf{U}_{2}^{\top}$ and let $\mathbf{T}_{2}=\mathbf{U}_{2}^{\top}$. Construct the transformation matrix $\mathbf{T}=$ $\mathbf{T}_{2} \mathbf{T}_{1}=\mathbf{U}_{2}^{\top} \mathbf{D}_{1}^{-\frac{1}{2}} \mathbf{U}_{1}^{\top}$.

2. Transforms estimates and covariances according to

$$
\begin{array}{ll}
\hat{\mathbf{z}}_{1}^{\prime}=\mathbf{T} \hat{\mathbf{z}}_{1}, & \mathbf{D}_{1}^{\prime}=\mathbf{T C}_{1} \mathbf{T}^{\top}=\mathbf{I}, \\
\hat{\mathbf{z}}_{2}^{\prime}=\mathbf{T} \hat{\mathbf{z}}_{2}, & \mathbf{D}_{2}^{\prime}=\mathbf{T C}_{2} \mathbf{T}^{\top} .
\end{array}
$$

3. For each component $i=1, \ldots, n_{x}$ of the vector $\hat{\mathbf{x}}^{\prime}$ and the diagonal matrix $\mathbf{P}^{\prime}$, calculate

$$
\left(\left[\hat{\mathbf{x}}^{\prime}\right]_{i},\left[\mathbf{P}^{\prime}\right]_{i i}\right)= \begin{cases}\left(\left[\hat{\mathbf{z}}_{1}^{\prime}\right]_{i},\left[\mathbf{D}_{1}^{\prime}\right]_{i i}\right), & \text { if }\left[\mathbf{D}_{1}^{\prime}\right]_{i i} \leq\left[\mathbf{D}_{2}^{\prime}\right]_{i i}, \\ \left(\left[\hat{\mathbf{z}}_{2}^{\prime}\right]_{i},\left[\mathbf{D}_{2}^{\prime}\right]_{i i}\right), & \text { if }\left[\mathbf{D}_{1}^{\prime}\right]_{i i}>\left[\mathbf{D}_{2}^{\prime}\right]_{i i} .\end{cases}
$$

4. Inverse transformation according to

$$
\hat{\mathbf{x}}=\mathbf{T}^{-1} \hat{\mathbf{x}}^{\prime}, \quad \mathbf{P}=\mathbf{T}^{-1} \mathbf{P}^{\prime} \mathbf{T}^{-\mathrm{T}} .
$$

Output: $\hat{\mathbf{x}}, \mathbf{P}$

The CLUE approach to the problem of fusion under component-wise aligned correlations, in case of $N=2$, is here formulated as the optimization problem

$$
\begin{array}{ll}
\mathbf{K}_{J}^{*}, \mathbf{P}^{*}=\underset{\substack{\mathbf{K}_{J}, \mathbf{P} \\
\text { subject to }}}{\arg \min } & \mathbf{P} \\
& \mathbf{K}_{J} \mathbf{H}_{J}=\mathbf{I} \\
& \mathbf{P} \geq \mathbf{K}_{J} \mathbf{C}_{J}^{\prime} \mathbf{K}_{J}^{\top}, \forall \mathbf{C}_{J}^{\prime} \in \mathbb{C},
\end{array}
$$

where

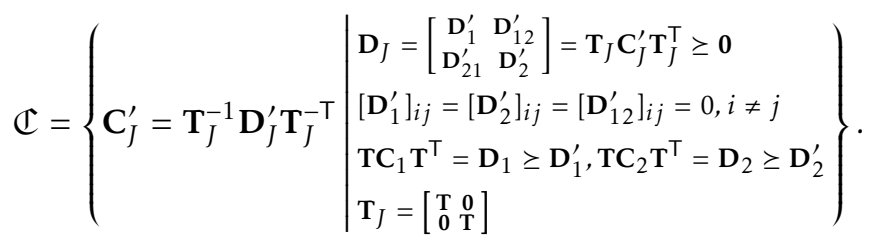

The LE method is provided in Algorithm 4.3. To the author's knowledge, there exists neither any variant of LE for the fusion of multiple estimates, nor any variant for the fusion of partial estimates. Below follows a modification of the LE method to be able to handle a partial estimate. 


\section{Algorithm 4.4: Largest Ellipsoid Method: Information Form}

Input: $\hat{\mathbf{z}}_{1}, \hat{\mathbf{z}}_{2}, \mathbf{C}_{1}, \mathbf{C}_{2}, \mathbf{H}_{1}, \mathbf{H}_{2}$

Assume $\operatorname{dim}\left(\hat{\mathbf{z}}_{1}\right)=n_{x}, \operatorname{dim}\left(\hat{\mathbf{z}}_{2}\right) \leq n_{x}$ and $\operatorname{rank}\left(\mathbf{H}_{1}\right)=n_{x}$. The estimates $\hat{\mathbf{z}}_{1}$ and $\hat{\mathbf{z}}_{2}$ are fused according to:

1. Transform into the information domain

$$
\begin{array}{ll}
\iota_{1}=\mathbf{H}_{1}^{\top} \mathbf{C}_{1}^{-1} \hat{\mathbf{z}}_{1}, & \mathcal{I}_{1}=\mathbf{H}_{1}^{\top} \mathbf{C}_{1}^{-1} \mathbf{H}_{1}, \\
\iota_{2}=\mathbf{H}_{2}^{\top} \mathbf{C}_{2}^{-1} \hat{\mathbf{z}}_{2}, & \mathcal{I}_{2}=\mathbf{H}_{2}^{\top} \mathbf{C}_{2}^{-1} \mathbf{H}_{2} .
\end{array}
$$

2. Decompose $\mathcal{I}_{1}=\mathbf{U}_{1} \mathbf{D}_{1}^{-1} \mathbf{U}_{1}^{\top}$ and let $\mathbf{T}_{1}=\mathbf{D}_{1}^{\frac{1}{2}} \mathbf{U}_{1}^{\top}$. Decompose $\mathbf{T}_{1} \mathcal{I}_{2} \mathbf{T}_{1}^{\top}=$ $\mathbf{U}_{2} \mathbf{D}_{2}^{+} \mathbf{U}_{2}^{\top}$, where the diagonal matrix $\mathbf{D}_{2}^{+}$might be singular, and let $\mathbf{T}_{2}=$ $\mathbf{U}_{2}^{\top}$. Construct the transformation matrix $\mathbf{T}=\mathbf{T}_{2} \mathbf{T}_{1}=\mathbf{U}_{2}^{\top} \mathbf{D}_{1}^{\frac{1}{2}} \mathbf{U}_{1}^{\top}$.

3. Transform the information states and information matrices according to

$$
\begin{array}{ll}
\iota_{1}^{\prime}=\mathbf{T} \iota_{1}, & \mathcal{I}_{1}^{\prime}=\mathbf{T} \mathcal{I}_{1} \mathbf{T}^{\top}, \\
\iota_{2}^{\prime}=\mathbf{T} \iota_{2}, & \mathcal{I}_{2}^{\prime}=\mathbf{T} \mathcal{I}_{2} \mathbf{T}^{\top} .
\end{array}
$$

4. For each (diagonal) component $i=1, \ldots, n_{x}$ of $\iota$ and $\mathcal{I}$, calculate

$$
\left([\iota]_{i},[\mathcal{I}]_{i i}\right)= \begin{cases}\left(\left[\iota_{1}^{\prime}\right]_{i},\left[\mathcal{I}_{1}^{\prime}\right]_{i i}\right), & \text { if }\left[\mathcal{I}_{1}^{\prime}\right]_{i i} \geq\left[\mathcal{I}_{2}^{\prime}\right]_{i i}, \\ \left(\left[\iota_{2}^{\prime}\right]_{i},\left[\mathcal{I}_{2}^{\prime}\right]_{i i}\right), & \text { if }\left[\mathcal{I}_{1}^{\prime}\right]_{i i}<\left[\mathcal{I}_{2}^{\prime}\right]_{i i} .\end{cases}
$$

5. Convert back to the covariance domain

$$
\mathbf{P}=\left(\mathbf{T}^{-1} \mathcal{I} \mathbf{T}^{-\mathrm{T}}\right)^{-1}, \quad \hat{\mathbf{x}}=\mathbf{P T}^{-1} \iota
$$

Output: $\hat{\mathbf{x}}, \mathbf{P}$

\section{Largest Ellipsoid Method for Partial Estimates}

A prerequisite for the LE method, as described in Algorithm 4.3, is that both covariance ellipsoids can be jointly transformed into axis aligned ellipsoids. In fact, it must be possible to transform one of the ellipsoids to the unit ball. If both covariance matrices are degenerate or if

$$
\operatorname{rank}\left(\mathbf{H}_{\imath}^{\top} \mathbf{C}_{\imath}^{-1} \mathbf{H}_{\imath}\right)<n_{x}, \quad \imath=1,2,
$$

then Algorithm 4.3 cannot be used anymore.

If $\operatorname{rank}\left(\mathbf{H}_{1}\right)=n_{x}$, it is possible to directly modify Algorithm 4.3 to handle one partial estimate. See Algorithm 4.4 for such adaption, where the information domain is once again utilized. Of course, an equivalent modification would be possible if instead $\operatorname{rank}\left(\mathbf{H}_{2}\right)=n_{x}$. 


\section{Properties}

An important feature of LE is that it provides the largest possible ellipsoid enclosed by the intersection [47]

$$
\mathcal{E}\left(\mathbf{0}, \mathbf{C}_{1}\right) \cap \mathcal{E}\left(\mathbf{0}, \mathbf{C}_{2}\right),
$$

which brings us to the subject of extremal volume ellipsoids [7]. Largest in this context refers to the volume of the ellipsoid, where the volume is proportional to the determinant of the underlying shape matrix. One type of extremal ellipsoids is the largest ellipsoid enclosed by a convex set [7], which is referred to as the inner Löwner-John ellipsoid ${ }^{3}$ (ILJE). A proper definition of the ILJE is given in Definition 4.1. The theory of extremal volume ellipsoid is interesting in this scope as explained below.

The intersection of (symmetric) convex sets is another (symmetric) convex set. Since ellipsoids are convex sets so is also the intersection of ellipsoids [31]. Assume that we have found an ILJE enclosed by a certain intersection of ellipsoids. By John's ellipsoid theorem [21], it is known that the minimum ellipsoid that encloses the same intersection will at maximum have a volume corresponding to $\mathcal{E}\left(\mathbf{0}, \mathbf{S}^{\mathrm{ILJE}}\right)$ scaled by a factor $\sqrt{n_{x}}$, where $n_{x}$ refers to the dimensionality. This observation can be used to find an ellipsoid that contains a complete intersection by finding the $\mathcal{E}\left(\mathbf{0}, \mathbf{S}^{\mathrm{ILJE}}\right)$ of that intersection.

Definition 4.1 (Inner Löwner-John Ellipsoid). Let $\mathcal{C} \in \mathbb{R}^{n}$ be an $n$-dimensional convex set centered at origin and let $S^{\mathrm{ILJE}} \in \mathfrak{S}^{++}$be given by

$$
\begin{array}{rlrl}
\mathbf{S}^{\mathrm{ILJE}}=\underset{\mathbf{S} \in \mathbb{R}^{n \times n}}{\arg \max } & \log (\operatorname{det}(\mathbf{S})) \\
\text { subject to } & \mathbf{x} \in \mathcal{C} \quad \forall \mathbf{x} \in \mathbb{R}^{n}, \mathbf{x}^{\top} \mathbf{S}^{-1} \mathbf{x} \leq 1 \\
& \mathbf{S} \in \mathfrak{S}^{++} . &
\end{array}
$$

The ILJE is then given by $\mathcal{E}\left(\mathbf{0}, \mathbf{S}^{\mathrm{ILJE}}\right)$.

In the derivation of EI another important aspect of LE is elucidated [60]. The information matrix $\left(\mathbf{P}^{\mathrm{LE}}\right)^{-1}$ of the fused estimate is the information given by adding $\mathbf{C}_{1}^{-1}$ and $\mathbf{C}_{2}^{-1}$, but with the removal of the maximum possible shared information $\left(\Gamma^{\mathrm{LE}}\right)^{-1}$, i.e.,

$$
\left(\mathbf{P}^{\mathrm{LE}}\right)^{-1}=\mathbf{C}_{1}^{-1}+\mathbf{C}_{2}^{-1}-\left(\Gamma^{\mathrm{LE}}\right)^{-1},
$$

which is similar to the fundamental idea behind ICI, where the shared information instead is bounded conservatively [49]. LE implicitly assumes the decomposition in (4.27). It has been shown that [49]

$$
\mathbf{P}^{\mathrm{ICI}}(\omega) \geq \mathbf{P}^{\mathrm{LE}},
$$

for all $\omega \in[0,1]$.

\footnotetext{
${ }^{3}$ This ellipsoid is often simply called the Löwner-John ellipsoid, but here the inner Löwner-John ellipsoid is used to separate it from its outer counterpart.
} 


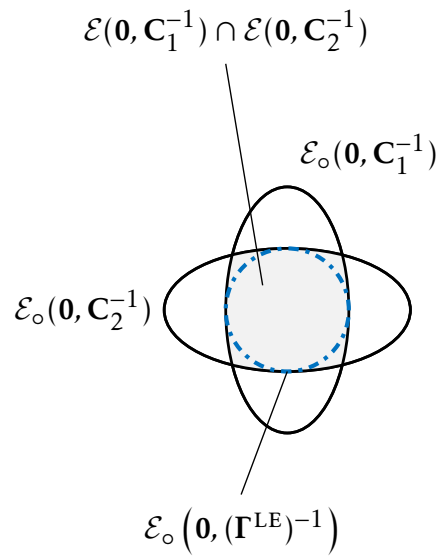

(a) Basic idea behind the LE method. LE calculates the potentially shared information having the maximum volume and subtracts this information.

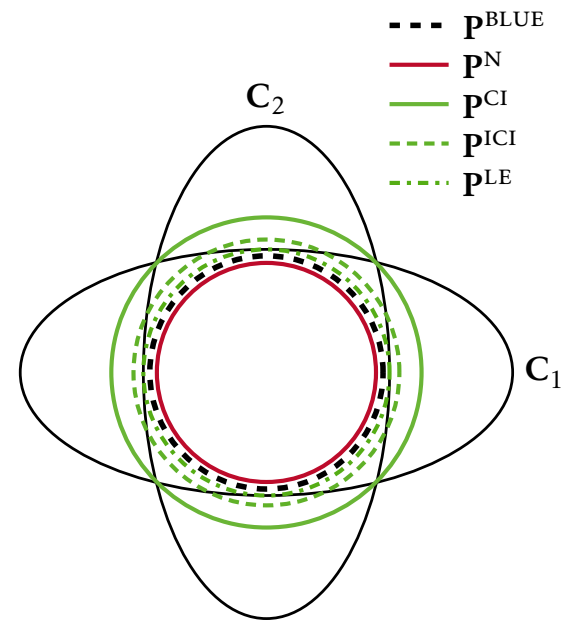

(b) Fusion of two estimates using the LE method.

Figure 4.4: The largest ellipsoid method.

The previously used fusion scenario with input covariances $\mathbf{C}_{1}$ and $\mathbf{C}_{2}$, now solved using by LE, is illustrated in Figure $4.4 \mathrm{~b}$.

Theorem 4.7 states that the LE estimator is a linear unbiased estimator. Theorem 4.8 shows that the LE method is a best CLUE if the cross-correlations obey the component-wise aligned structure.

Theorem 4.7 (Largest Ellipsoid Method-Linear Unbiased). As given in Algorithm 4.3, the largest ellipsoid method for fusion of $\hat{\mathbf{z}}_{1}=\mathbf{x}+\mathbf{e}_{1}$ and $\hat{\mathbf{z}}_{2}=\mathbf{x}+\mathbf{e}_{2}$, where $\mathbf{C}_{1} \geq \operatorname{cov}\left(\hat{\mathbf{z}}_{1}\right)$ and $\mathbf{C}_{2} \geq \operatorname{cov}\left(\hat{\mathbf{z}}_{2}\right)$, is a linear unbiased estimator.

Proof: It is assumed $\mathbf{H}_{1}=\mathbf{H}_{2}=\mathbf{I}$. The unbiased and linear properties are checked by showing the LE method can be expressed using the fusion gains $\mathbf{K}_{1}$ and $\mathbf{K}_{2}$ such that

$$
\hat{\mathbf{x}}^{\mathrm{LE}}=\mathbf{K}_{1} \hat{\mathbf{z}}_{1}+\mathbf{K}_{2} \hat{\mathbf{z}}_{2}, \quad \mathbf{P}^{\mathrm{LE}}=\mathbf{K}_{1} \mathbf{C}_{1} \mathbf{K}_{1}^{\top}+\mathbf{K}_{2} \mathbf{C}_{2} \mathbf{K}_{2}^{\top},
$$

while fulfilling $\mathbf{K}_{1}+\mathbf{K}_{2}=\mathbf{I}$.

The LE method is based on the transformation

$$
\left[\begin{array}{c}
\hat{\mathbf{z}}_{1}^{\prime} \\
\hat{\mathbf{z}}_{2}^{\prime}
\end{array}\right]=\mathbf{T}_{J}\left[\begin{array}{c}
\hat{\mathbf{z}}_{1} \\
\hat{\mathbf{z}}_{2}
\end{array}\right], \quad\left[\begin{array}{cc}
\mathbf{I} & \mathbf{0} \\
\mathbf{0} & \mathbf{C}_{2}^{\prime}
\end{array}\right]=\mathbf{T}_{J}\left[\begin{array}{cc}
\mathbf{C}_{1} & \mathbf{0} \\
\mathbf{0} & \mathbf{C}_{2}
\end{array}\right] \mathbf{T}_{J}^{\top},
$$

where $\mathbf{T}_{J}=\operatorname{diag}(\mathbf{T}, \mathbf{T})$ is the joint transformation matrix with $\mathbf{T}$ defined as in Algorithm 4.3. In the transformed domain the gains $\mathbf{K}_{1}^{\prime}$ and $\mathbf{K}_{2}^{\prime}$ can be constructed 
as

$$
\mathbf{K}_{1}^{\prime}=\operatorname{diag}\left(k_{1}, \ldots, k_{n_{x}}\right), \quad \quad \mathbf{K}_{2}^{\prime}=\mathbf{I}-\mathbf{K}_{1}^{\prime},
$$

where $k_{i}=1$ if the $i$ th component is picked from $\hat{\mathbf{z}}_{1}^{\prime}$, otherwise $k_{i}=0$. Clearly $\mathbf{K}_{1}^{\prime}+\mathbf{K}_{2}^{\prime}=\mathbf{I}$. In the original domain the estimator is linear since

$$
\hat{\mathbf{x}}^{\mathrm{LE}}=\mathbf{T}^{-1}\left(\mathbf{K}_{1}^{\prime} \hat{\mathbf{z}}_{1}^{\prime}+\mathbf{K}_{2}^{\prime} \hat{\mathbf{z}}_{2}^{\prime}\right)=\mathbf{T}^{-1}\left(\mathbf{K}_{1}^{\prime} \mathbf{T} \hat{\mathbf{z}}_{1}+\mathbf{K}_{2}^{\prime} \mathbf{T} \hat{\mathbf{z}}_{1}\right)=\mathbf{K}_{1} \hat{\mathbf{z}}_{1}+\mathbf{K}_{2} \hat{\mathbf{z}}_{2},
$$

where $\mathbf{K}_{1}=\mathbf{T}^{-1} \mathbf{K}_{1}^{\prime} \mathbf{T}$ and $\mathbf{K}_{2}=\mathbf{T}^{-1} \mathbf{K}_{2}^{\prime} \mathbf{T}$. We also see that

$$
\mathbf{K}_{1}+\mathbf{K}_{2}=\mathbf{T}^{-1}\left(\mathbf{K}_{1}^{\prime}+\mathbf{K}_{2}^{\prime}\right) \mathbf{T}=\mathbf{T}^{-1} \mathbf{T}=\mathbf{I}
$$

Theorem 4.8 (Largest Ellipsoid Method-Optimal). Assume $\hat{\mathbf{z}}_{1}=\mathbf{x}+\mathbf{e}_{1}$ and $\hat{\mathbf{z}}_{2}=\mathbf{x}+\mathbf{e}_{2}$ are given, where $\mathbf{C}_{1} \geq \operatorname{cov}\left(\hat{\mathbf{z}}_{1}\right)$ and $\mathbf{C}_{2} \geq \operatorname{cov}\left(\hat{\mathbf{z}}_{2}\right)$. If the crosscorrelations follow the component-wise aligned correlation structure of (4.52), then the largest ellipsoid method as given by Algorithm 4.3 is a solution to (4.53) and hence is a best conservative linear unbiased estimator.

Proof: The joint covariance after joint transformation $\mathbf{T}_{J}=\operatorname{diag}(\mathbf{T}, \mathbf{T})$ is given by

$$
\mathbf{D}_{J}=\mathbf{T}_{J} \mathbf{C}_{J} \mathbf{T}_{J}^{\top}=\left[\begin{array}{cc}
\mathbf{D}_{1} & \mathbf{D}_{12} \\
\mathbf{D}_{12} & \mathbf{D}_{2}
\end{array}\right],
$$

where $\mathbf{D}_{1}=\mathbf{I}>\mathbf{0}, \mathbf{D}_{2}>\mathbf{0}$ and $\mathbf{D}_{12}$ are diagonal. The $i$ th component of $\mathbf{D}_{1}$ is only correlated with the $i$ th component of $\mathbf{D}_{2}$. Hence, we only need to consider correlated scalars. It is then possible to use CI for the merging of correlated scalars which are correlated to an unknown degree. If $\mathbf{D}^{\mathrm{LE}}$ is the covariance of the $\mathrm{LE}$ estimate in the transformed domain we get

$$
\left[\mathbf{D}^{\mathrm{LE}}\right]_{i i}=\omega\left[\mathbf{D}_{1}\right]_{i i}+(1-\omega)\left[\mathbf{D}_{2}\right]_{i i}=\omega+(1-\omega)\left[\mathbf{D}_{2}\right]_{i i},
$$

which is conservative for all $\omega=[0,1]$. Minimizing $\left[\mathbf{D}^{\mathrm{LE}}\right]_{i i}$ w.r.t. $\omega$ is equivalent to

$$
\left[\mathbf{D}^{\mathrm{LE}}\right]_{i i}=\min \left(1,\left[\mathbf{D}_{2}\right]_{i i}\right),
$$

which in particular is the LE solution. Theorem 4.7 shows that LE is a linear unbiased estimator. The optimality proof in [58] shows that CI is a best CLUE. Hence, LE is a best CLUE given Theorem 4.7 and [58].

Assuming the component-wise aligned structure the information matrix of the BLUE estimate in the transformed domain is given by

$$
\begin{aligned}
\left(\mathbf{D}^{\mathrm{BLUE}}\right)^{-1} & =\left[\begin{array}{ll}
\mathbf{I} & \mathbf{I}
\end{array}\right] \mathbf{D}_{J}^{-1}\left[\begin{array}{l}
\mathbf{I} \\
\mathbf{I}
\end{array}\right] \\
& =\mathbf{I}+\left(\mathbf{D}_{12}-\mathbf{I}\right)\left(\mathbf{D}_{2}-\mathbf{D}_{12}^{2}\right)^{-1}\left(\mathbf{D}_{12}-\mathbf{I}\right) \\
& =\mathbf{D}_{2}^{-1}+\left(\mathbf{D}_{2}^{-1} \mathbf{D}_{12}-\mathbf{I}\right)\left(\mathbf{I}-\mathbf{D}_{12} \mathbf{D}_{2}^{-1} \mathbf{D}_{12}\right)^{-1}\left(\mathbf{D}_{12} \mathbf{D}_{2}^{-1}-\mathbf{I}\right),
\end{aligned}
$$


which is a diagonal matrix since each of the matrices on the r.h.s. is diagonal. Hence, the $i$ th diagonal component of $\left(\mathbf{D}^{\mathrm{BLUE}}\right)^{-1}$ is given by

$$
\begin{aligned}
{\left[\left(\mathbf{D}^{\mathrm{BLUE}}\right)^{-1}\right]_{i i} } & =1+\frac{\left(\left[\mathbf{D}_{12}\right]_{i i}-1\right)^{-1}}{\left[\mathbf{D}_{2}\right]_{i i}-\left[\mathbf{D}_{12}\right]_{i i}^{2}} \\
& =\left[\mathbf{D}_{2}^{-1}\right]_{i i}+\frac{\left(\left[\mathbf{D}_{2}^{-1}\right]_{i i}\left[\mathbf{D}_{12}\right]_{i i}-1\right)^{-1}}{1-\left[\mathbf{D}_{12}\right]_{i i}^{2}\left[\mathbf{D}_{2}^{-1}\right]_{i i}}
\end{aligned}
$$

At the same time, the $i$ th component of the LE information matrix $\left(D^{L E}\right)^{-1}$ in the transformed domain, which also is diagonal, is

$$
\left[\left(\mathbf{D}^{\mathrm{LE}}\right)^{-1}\right]_{i i}=\max \left(1,\left[\mathbf{D}_{2}^{-1}\right]_{i i}\right)
$$

By pure inspection it can be seen that the cross-covariance $\mathbf{D}_{12}$ that gives $(4.60)$ equal to (4.61), i.e., $\mathbf{D}^{\mathrm{BLUE}}=\mathbf{D}^{\mathrm{LE}}$, is given by

$$
\left[\mathbf{D}_{12}\right]_{i i}=\min \left(1,\left[\mathbf{D}_{2}\right]_{i i}\right) \text {, }
$$

which means the LE method implicitly assumes the joint covariance

$$
\mathbf{C}_{J}^{\mathrm{LE}}=\left[\begin{array}{cc}
\mathbf{C}_{1} & \mathbf{T}^{-1} \mathbf{D}_{12} \mathbf{T}^{-\mathrm{T}} \\
\mathbf{T}^{-\mathrm{T}} \mathbf{D}_{12} \mathbf{T}^{-1} & \mathbf{C}_{2}
\end{array}\right] .
$$

The LE method can hence alternatively be expressed using the WLS formula with $\mathbf{H}_{J}=\left[\begin{array}{ll}\mathbf{H}_{1}^{\top} & \mathbf{H}_{2}^{\top}\end{array}\right]^{\top}$ as

$$
\begin{aligned}
\hat{\mathbf{x}}^{\mathrm{LE}} & =\left(\mathbf{H}_{J}^{\top}\left(\mathbf{C}_{J}^{\mathrm{LE}}\right)^{-1} \mathbf{H}_{J}\right)^{-1} \mathbf{H}_{J}^{\top}\left(\mathbf{C}_{J}^{\mathrm{LE}}\right)^{-1} \hat{\mathbf{z}}_{J}, \\
\mathbf{P}^{\mathrm{LE}} & =\left(\mathbf{H}_{J}^{\top}\left(\mathbf{C}_{J}^{\mathrm{LE}}\right)^{-1} \mathbf{H}_{J}\right)^{-1},
\end{aligned}
$$

where $\operatorname{rank}\left(\mathbf{H}_{1}\right)=\operatorname{rank}\left(\mathbf{H}_{2}\right)=n_{x}$. The estimate $\hat{\mathbf{x}}^{\mathrm{LE}}$ in this case is calculated as a weighted mean instead of choosing each component of $\hat{\mathbf{x}}^{\mathrm{LE}}$ exclusively from either of $\hat{\mathbf{z}}_{1}$ or $\hat{\mathbf{z}}_{2}$ like what is done in Algorithm 4.3.

The joint covariance assumed in CI and ICI are conservative bounds on $\mathbf{C}_{J}$ under different assumptions and parametrizations of the cross-correlations. The joint covariance of (4.63) instead assumes a specific cross-covariance $\mathrm{C}_{12}$ that gives the largest ellipsoid enclosed in the intersection $\mathcal{E}\left(\mathbf{0}, \mathbf{C}_{1}\right) \cap \mathcal{E}\left(\mathbf{0}, \mathbf{C}_{2}\right)$ [47].

\subsection{Summary}

The previously defined conservative fusion methods are compared in Figure 4.5. In this case it is clear that

$$
\mathcal{E}\left(\mathbf{0}, \mathbf{P}^{\mathrm{N}}\right) \subset \mathcal{E}\left(\mathbf{0}, \mathbf{P}^{\mathrm{WLS}}\right) \subset \mathcal{E}\left(\mathbf{0}, \mathbf{P}^{\mathrm{LE}}\right) \subset \mathcal{E}\left(\mathbf{0}, \mathbf{P}^{\mathrm{ICI}}\right) \subset \mathcal{E}\left(\mathbf{0}, \mathbf{P}^{\mathrm{CI}}\right) .
$$




$$
\begin{aligned}
& -\cdots\left(\mathbf{P}^{\mathrm{BLUE}}\right)^{-1} \\
& -\left(\mathbf{P}^{\mathrm{N}}\right)^{-1} \\
& -\left(\mathbf{P}^{\mathrm{CI}}\right)^{-1} \\
& -\cdots=\left(\mathbf{P}^{\mathrm{ICI}}\right)^{-1} \\
& =\cdots=\left(\mathbf{P}^{\mathrm{LE}}\right)^{-1}
\end{aligned}
$$

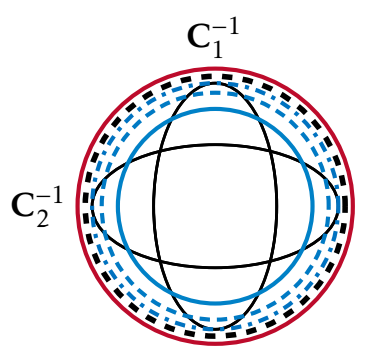

(a) Information domain.

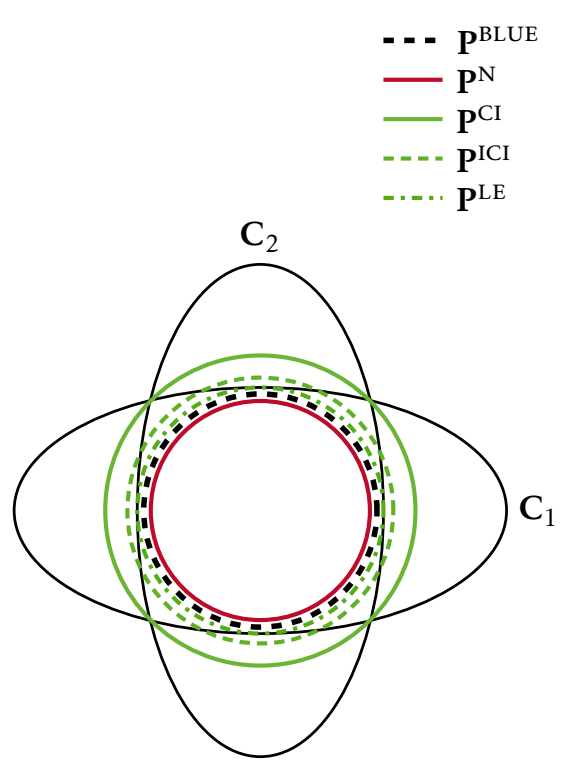

(b) Covariance domain.

Figure 4.5: Comparison of the different conservative fusion methods described in this chapter.

However, in general

$$
\mathcal{E}\left(\mathbf{0}, \mathbf{P}^{\mathrm{LE}}\right) \subseteq \mathcal{E}\left(\mathbf{0}, \mathbf{P}^{\mathrm{ICI}}\right) \subseteq \mathcal{E}\left(\mathbf{0}, \mathbf{P}^{\mathrm{CI}}\right)
$$

It must be emphasized that $\mathcal{E}\left(\mathbf{0}, \mathbf{P}^{\mathrm{ICI}}\right) \subseteq \mathcal{E}\left(\mathbf{0}, \mathbf{P}^{\mathrm{CI}}\right)$, or equivalently $\mathrm{P}^{\mathrm{ICI}} \leq \mathbf{P}^{\mathrm{CI}}$, is in the parametric sense as described in Section 3.4.2. Denoting $\omega^{\mathrm{ICI}} \in[0,1]$ the free parameter of $\mathbf{P}^{\mathrm{ICI}}=\mathbf{P}^{\mathrm{ICI}}\left(\omega^{\mathrm{ICI}}\right)$ and $\omega^{\mathrm{CI}} \in[0,1]$ the free parameter of $\mathbf{P}^{\mathrm{CI}}=\mathbf{P}^{\mathrm{CI}}\left(\omega^{\mathrm{CI}}\right)$, then by $\mathbf{P}^{\mathrm{CI}} \geq \mathbf{P}^{\mathrm{ICI}}$ we mean that for each $\omega^{\mathrm{CI}}=\omega_{0}^{\mathrm{CI}}$ there exists at least one $\omega^{\mathrm{ICI}}=\omega_{0}^{\mathrm{ICI}}$ such that

$$
\mathbf{P}^{\mathrm{CI}}\left(\omega_{0}^{\mathrm{CI}}\right) \geq \mathbf{P}^{\mathrm{ICI}}\left(\omega_{0}^{\mathrm{ICI}}\right)
$$

As noted earlier, in [49] it is shown that

$$
\mathbf{P}^{\mathrm{ICI}}\left(\omega^{\mathrm{ICI}}\right) \geq \mathbf{P}^{\mathrm{LE}},
$$

for all $\omega^{\mathrm{ICI}} \in[0,1]$. This should not come as a surprise since ICI in its original derivation and LE are based on the assumption that the information of input estimates can be decomposed into one term of exclusive information and one term of common information. While in LE the maximum possible common information $\left(\Gamma^{\mathrm{LE}}\right)^{-1}$ is calculated and then subtracted, in ICI all possibilities on the common information are bounded by $\left(\boldsymbol{\Gamma}^{\mathrm{ICI}}\right)^{-1}=\left(\omega^{\mathrm{ICI}} \mathbf{C}_{1}+\left(1-\omega^{\mathrm{ICI}}\right) \mathbf{C}_{2}\right)^{-1}$ which is then subtracted, i.e., $\left(\Gamma^{\mathrm{ICI}}\right)^{-1} \geq\left(\Gamma^{\mathrm{LE}}\right)^{-1}$ or equivalently

$$
\left(\mathbf{P}^{\mathrm{LE}}\right)^{-1}=\mathbf{C}_{1}^{-1}+\mathbf{C}_{2}^{-1}-\left(\boldsymbol{\Gamma}^{\mathrm{LE}}\right)^{-1} \geq \mathbf{C}_{1}^{-1}+\mathbf{C}_{2}^{-1}-\left(\boldsymbol{\Gamma}^{\mathrm{ICI}}\right)^{-1}=\left(\mathbf{P}^{\mathrm{ICI}}\right)^{-1} .
$$




\section{Covariance intersection}

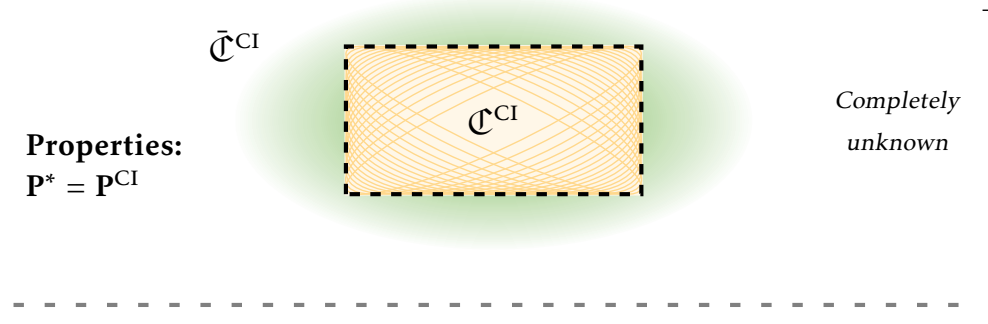

\section{Inverse covariance intersection}

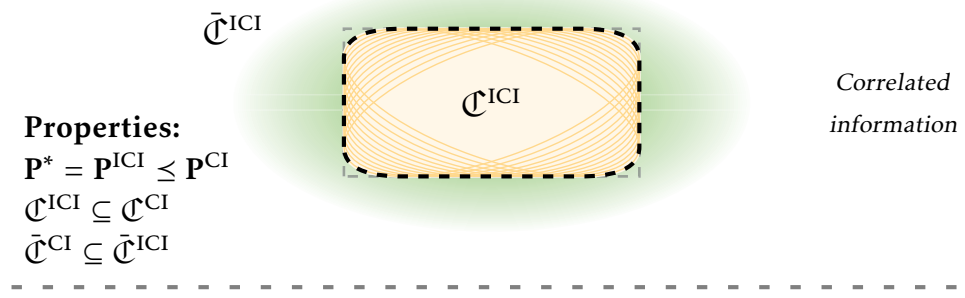

\section{Largest ellipsoid method}

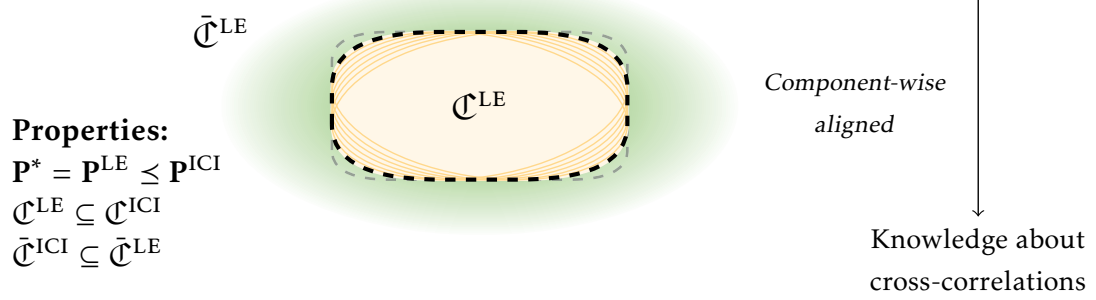

Figure 4.6: Illustration of the conservative fusion methods presented in this chapter. More knowledge about the cross-correlations available means that $\mathbb{C}$ will be smaller (and that $\overline{\mathbb{C}}$ will be larger) and hence the optimal $\mathbf{P}^{*}$ becomes smaller.

The ordering of

$$
\mathbf{P}^{\mathrm{CI}} \geq \mathbf{P}^{\mathrm{ICI}} \geq \mathbf{P}^{\mathrm{LE}},
$$

is directly related to another important fact, viz.,

$$
\mathbb{C}^{\mathrm{LE}} \subseteq \mathbb{C}^{\mathrm{ICI}} \subseteq \mathbb{C}^{\mathrm{CI}}
$$

where $\mathbb{C}^{\mathrm{LE}}, \mathbb{C}^{\mathrm{ICI}}$ and $\mathbb{C}^{\mathrm{CI}}$ refer to the set $\mathbb{C}$ as given by (4.54), (4.34a) and (4.14a), respectively. The relationship in (4.71) follows from the different assumptions on the cross-correlation, i.e., LE assumes stronger conditions on the cross-correlations than does ICI, and ICI assumes stronger conditions on the cross-correlation than does CI. Likewise we have

$$
\overline{\mathbb{C}}^{\mathrm{CI}} \subseteq \overline{\mathbb{C}}^{\mathrm{ICI}} \subseteq \overline{\mathbb{C}}^{\mathrm{LE}}
$$


where $\overline{\mathbb{C}}^{\mathrm{LE}}, \overline{\mathbb{C}}^{\mathrm{ICI}}$ and $\overline{\mathbb{C}}^{\mathrm{CI}}$ are the sets corresponding to $\overline{\mathbb{C}}$ in case of LE, ICI and $\mathrm{CI}$, respectively. We summarize these observations in Figure 4.6. The figure is however not realistic, since if $\mathbf{C}_{J}$ is $2 \times 2$ the cross-covariance is a scalar and hence $\mathbb{C}^{\mathrm{LE}}=\mathbb{C}^{\mathrm{ICI}}=\mathbb{C}^{\mathrm{CI}}$, but the figure nevertheless illustrates what more knowledge about the cross-correlations in practice means. 


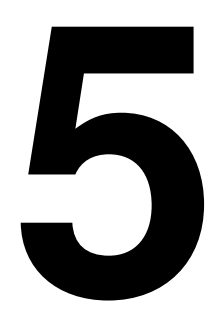

\section{Diagonal Covariance Approximation}

$\mathrm{T}$

HE DATALINK is an integral part of a decentralized sensor network since estimates must be interchanged between the nodes of the network. Even though the communication bandwidth is reduced by transmission of aggregated estimates instead of measurements, it is still often crucial to use the datalink in an effective way [30].

The estimates to be interchanged are given on the form $\hat{\mathbf{z}}_{l}=\mathbf{H}_{l} \mathbf{x}+\mathbf{e}_{l}$ where $\mathbf{x}$ is the true state, $\mathbf{H}_{l}$ is the mapping from the true state $\mathcal{X}$ to $\mathcal{Z}_{l}, \mathbf{e}_{l}$ is zero-mean noise and $\mathbf{C}_{l} \geq \operatorname{cov}\left(\hat{\mathbf{z}}_{l}\right)$ is the reported covariance of the conservative estimate $\hat{\mathbf{z}}_{l}$. Since $\mathbf{C}_{l}$ is a matrix it scales as $n_{l}^{2}$ where $n_{l}=\operatorname{dim}\left(\hat{\mathbf{z}}_{l}\right)$. It is therefore tempting to only transmit a subset of the elements of the covariance matrices to reduce the data exchanged on the datalink.

In this chapter the diagonal covariance approximation (DCA) is used as a data reduction technique [12]. The DCA approximates a covariance matrix $\mathbf{C}_{l}$ by a diagonal matrix which in practice means the number of elements transmitted is $n_{l}$. Care however needs to be taken such that conservativeness of the estimates is still ensured.

The problem is stated in Section 5.1 and different methods for preservation of conservative estimates are given in Section 5.2. A simulation evaluation is finally done in Section 5.3.

The contents of this chapter has previously been published in:

Robin Forsling, Zoran Sjanic, Fredrik Gustafsson, and Gustaf Hendeby. Consistent distributed track fusion under communication constraints. In Proceedings of the 22nd IEEE International Conference on Information Fusion, Ottawa, Canada, July 2019.

Compared to [12] this chapter uses another notation and a slightly modified simulation scenario. 


\subsection{Problem Formulation}

In decentralized fusion problems the common setting is to fuse a local conservative estimate with conservative estimates received from other nodes of the network using an estimation method that is robust to potential cross-correlations between the estimates. The goal is to calculate a conservative estimate $\hat{\mathbf{x}}$ with a covariance $\mathbf{P} \geq \operatorname{cov}(\hat{\mathbf{x}})$. This problem is here further extended to include the setup where the DCA is used to reduce the bandwidth utilization.

Estimates to be fused are given by $\hat{\mathbf{z}}_{\imath}=\mathbf{H}_{\imath} \mathbf{x}+\mathbf{e}_{\imath}$ with $\imath=1, \ldots, N$ where $N$ is the number of estimates to be fused. When transmitting $\hat{\mathbf{z}}_{\imath}$ the covariance $\mathbf{C}_{l}$ will be approximated by $\mathbf{D}_{\imath}$ which is a diagonal matrix, hence the name DCA. Assume $\mathbf{C}_{\imath}>\mathbf{0}$ and $\mathbf{D}_{\imath}>\mathbf{0}$ for all $\imath=1, \ldots, N$.

The CI algorithm will be used as the primary conservative fusion technique in this problem. CI yields a conservatively fused estimate for any degree of crosscorrelations as long as the estimates to be fused are conservative. Section 4.1.2 describes the CI method in more detail. Next, implications of the DCA are demonstrated.

\subsubsection{Consequence of the Diagonal Covariance Approximation}

A conservative estimate $\hat{\mathbf{z}}$ where the covariance $\mathbf{C} \geq \operatorname{cov}(\hat{\mathbf{z}})$ is approximated by $\mathbf{C}^{\prime}$ is conservative if

$$
\mathrm{C}^{\prime}-\mathrm{C} \geq \mathbf{0},
$$

since this $\mathbf{C}^{\prime}$ will automatically satisfy the condition in (3.18), i.e., if (5.1) holds then

$$
\mathbf{C} \geq \operatorname{cov}(\hat{\mathbf{z}}) \Longrightarrow \mathbf{C}^{\prime} \geq \operatorname{cov}(\hat{\mathbf{z}}),
$$

where $\operatorname{cov}(\hat{\mathbf{z}})$ refers to the true covariance of the estimate $\hat{\mathbf{z}}$.

Approximating the full covariance matrix $\mathbf{C}$ by its diagonal entries will in general generate an estimate which is not conservative. For example, consider $\mathbf{C}$ defined by

$$
\mathbf{C}=\left[\begin{array}{ll}
a & b \\
b & d
\end{array}\right]>0,
$$

and introduce the DCA $\mathbf{D}$ given by

$$
\mathbf{D}=\left[\begin{array}{ll}
a & 0 \\
0 & d
\end{array}\right]>0 .
$$

The characteristic equation for the matrix on the 1.h.s. of (5.1) with $\mathbf{D}$ replacing $\mathrm{C}^{\prime}$ is

$$
\operatorname{det}(\lambda \mathbf{I}-(\mathbf{D}-\mathbf{C}))=\lambda^{2}-b^{2}=0,
$$

which gives the eigenvalues $\lambda= \pm b$. Hence, the matrix $\mathbf{D}-\mathbf{C}$ is indefinite. To guarantee conservative estimates the DCA must be accompanied by some mechanism for preservation of conservativeness. 


\subsection{Methods for Preservation of Conservative Estimates}

In this section $N=2$ estimates will assumed, where $N$ refers to the number of nodes or estimates. The linear model of (2.40) is assumed.

A local estimate is given by $\hat{\mathbf{z}}_{\ell}=\mathbf{H}_{\ell} \mathbf{x}+\mathbf{e}_{\ell}$ where $\mathbf{C}_{\ell} \geq \operatorname{cov}\left(\hat{\mathbf{z}}_{\ell}\right)$. Available at another node is the estimate $\hat{\mathbf{z}}$ for which the subscripted index has been dropped to simplify the notation. The problem is that $\mathbf{C} \geq \operatorname{cov}(\hat{\mathbf{z}})$ is unavailable, but instead a DCA $\mathbf{D}^{\prime}$ is available. $\mathbf{D}^{\prime}$ is either $\mathbf{D}$ given by

$$
[\mathbf{D}]_{i j}= \begin{cases}{[\mathbf{C}]_{i j},} & \text { if } i=j, \\ 0, & \text { if } i \neq j,\end{cases}
$$

or a diagonal conservative bound $\overline{\mathbf{D}}$. Let $\mathbb{C}$ be the set containing all matrices which would generate the same $\mathbf{D}$ using the formula of (5.3). Then for the conservative bound $\overline{\mathbf{D}}$ it must hold that $\overline{\mathbf{D}} \geq \mathbf{C}^{\prime}, \forall \mathbf{C}^{\prime} \in \mathbb{C}$.

In total five methods for preservation of conservativeness are proposed in this section. Two of the methods can be implemented at the receiving node-these are called post-transmission methods. The remaining three methods must be implemented at the transmitting node and hence will be named pre-transmission methods. The majority of the methods are based on scaling, i.e., inflation of $\mathbf{D}$ in some way.

The following methods are considered:

- Post-transmission handling of DCA using an adaption of CI (POST-CI).

- Post-transmission handling of DCA using scaling (POST-SCALE).

- Pre-transmission handling of DCA using eigenvalue-based scaling (PRE-EIG).

- Pre-transmission handling of DCA using optimization-based scaling (PREOPT).

- Pre-transmission handling of DCA using diagonal-dominance scaling (PREDOM).

\subsubsection{Post-Transmission Adaption of Covariance Intersection}

Assume that two conservative estimates $\hat{\mathbf{z}}_{\ell}$ and $\hat{\mathbf{z}}$ to be fused are given, where $\mathbf{C}_{\ell}$ is provided but where only the diagonal entries of $\mathbf{C}$ are known, i.e., D. Assume $\operatorname{dim}\left(\hat{\mathbf{z}}_{\ell}\right)=\operatorname{dim}(\hat{\mathbf{z}})=n_{x}$ and $\mathbf{H}_{\ell}=\mathbf{H}=\mathbf{I}$.

Direct application of CI cannot be done if a conservative fused estimate must be guaranteed. However, the $i$ th component of $\hat{\mathbf{z}}$ is a scalar estimate $\hat{z}_{i}$ of the $i$ th component of $\mathbf{x}$ having the variance $[\mathbf{D}]_{i i}=\sigma_{i}^{2}$. Each of these $n_{x}$ scalar estimates will also be conservative since $\hat{\mathbf{z}}$ is conservative. The POST-CI method is expressed 


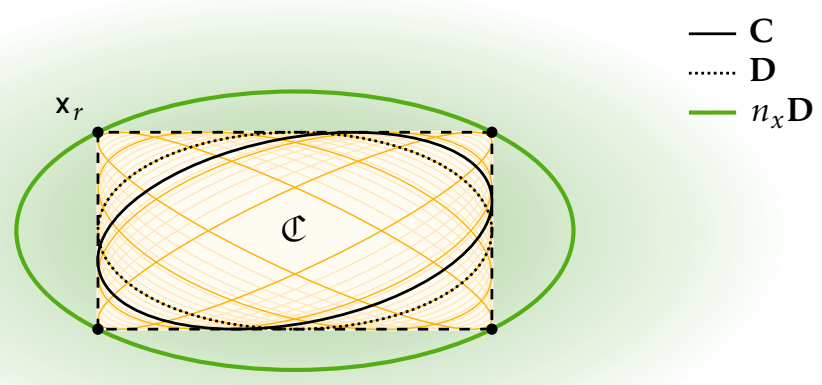

Figure 5.1: The axis-aligned ellipse within the rectangle, $\mathbf{D}$, is the DCA of $\mathbf{C}$. The ellipse of $n_{x} \mathbf{D}$ encloses all ellipses of $\mathbf{C}^{\prime} \in \mathbb{C}$. The ellipses of $\mathbf{C}^{\prime} \in \mathbb{C}$ are bounded from above by the dashed rectangle.

using (4.21) as

$$
\begin{gathered}
\mathbf{P}^{-1}=\omega_{\ell} \mathbf{C}_{\ell}^{-1}+\sum_{i=1}^{n_{x}} \omega_{i} \mathbf{H}_{i}^{\top} \sigma_{i}^{-2} \mathbf{H}_{i}, \\
\mathbf{P}^{-1} \hat{\mathbf{x}}=\omega_{\ell} \mathbf{C}_{\ell}^{-1} \hat{\mathbf{z}}_{\ell}+\sum_{i=1}^{n_{x}} \omega_{i} \mathbf{H}_{i}^{\top} \sigma_{i}^{-2} \hat{z}_{i},
\end{gathered}
$$

where $\omega_{\ell}, \omega_{i} \in[0,1], \omega_{\ell}+\sum_{i=1}^{n_{x}} \omega_{i}=1$ and the $1 \times n_{x}$ matrices $\mathbf{H}_{i}$ are given by

$$
\mathbf{H}_{i}=\left[\begin{array}{llll}
\delta_{i 1} & \delta_{i 2} & \ldots & \delta_{i n_{x}}
\end{array}\right],
$$

where $\delta_{i j}$ is the Kronecker delta.

\subsubsection{Post-Transmission Scaling}

Upon reception of $\mathbf{D}$ given by (5.3) it will be impossible to reconstruct the original covariance matrix $\mathbf{C}$ since it will only be known $\mathbf{C} \in \mathbb{C}$, where the set $\mathbb{C}$ includes all matrices $\mathbf{C}^{\prime}>\mathbf{0}$ that $\mathbf{C}$ can take given $\mathbf{D}$.

A conservative bound $\overline{\mathbf{D}}$ satisfying $\overline{\mathbf{D}} \geq \mathbf{C}^{\prime}, \forall \mathbf{C}^{\prime} \in \mathbb{C}$ should be used in replacement of D. Looking at Figure 5.1 it can be seen that an axis-aligned ellipse which intersects the point $\mathbf{x}_{r}$ will enclose all matrices $\mathbf{C}^{\prime} \in \mathbb{C}$ which are contained in the dashed rectangle. One such ellipse, i.e., a conservative bound, is given by the ellipse of $n_{x} \mathbf{D}$. From Chapter 3 we know that there exists an infinite number of conservative bounds $\overline{\mathbf{C}} \in \overline{\mathbb{C}}$ that passes through $\mathrm{x}_{r}$, where the set $\overline{\mathbb{C}}$ contains all $\overline{\mathbf{C}}$ for which $\overline{\mathbf{C}} \geq \mathbf{C}^{\prime}, \forall \mathbf{C}^{\prime} \in \mathbb{C}$ hold. For this method only $n_{x} \mathbf{D}$ will be considered.

That POST-SCALE preserves conservative estimates is shown in Theorem 5.1. Using the concept of Löwner-John ellipsoids that were presented in Section 4.3.2 we see that $\mathbf{D}$ and $n_{x} \mathbf{D}$ refer to the inner and outer Löwner-John, respectively. In 
this case the convex set enclosing the inner Löwner-John is given by a hyperrectangle [7].

Theorem 5.1. Let $\mathbf{C} \geq \mathbf{0}$ be a $n_{x} \times n_{x}$ matrix and $\mathbf{D} \geq \mathbf{0}$ the diagonal matrix given by

$$
[\mathbf{D}]_{i j}= \begin{cases}{[\mathbf{C}]_{i j},} & \text { if } i=j, \\ 0, & \text { if } i \neq j .\end{cases}
$$

Then $n_{x} \mathbf{D} \geq \mathbf{C}$.

Proof: If $n_{x} \mathbf{D}-\mathbf{C} \geq \mathbf{0}$ then the following is true

$$
\begin{aligned}
& c \mathbf{D}-\mathbf{P} \geq \mathbf{0}, \\
\Longleftrightarrow & c \mathbf{D}^{-\frac{1}{2}} \mathbf{D D}^{-\frac{1}{2}}-\mathbf{D}^{-\frac{1}{2}} \mathbf{P D}^{-\frac{1}{2}} \geq \mathbf{0}, \\
\Longleftrightarrow & c \mathbf{I}-\mathbf{Q} \geq \mathbf{0},
\end{aligned}
$$

where $\mathbf{I}$ is the identity matrix and $\mathbf{Q}$ is the correlation matrix having ones on its diagonal and the off-diagonal elements are bounded as $\left|q_{i j}\right| \leq 1$. Hence, $n_{x} \mathbf{I}-\mathbf{Q}$ becomes a symmetric diagonally dominant matrix and we get $n_{x} \mathbf{I}-\mathbf{Q} \geq \mathbf{0} \Longleftrightarrow$ $n_{x} \mathbf{D}-\mathbf{C} \geq \mathbf{0}[19]$.

\subsubsection{Pre-Transmission Eigenvalue-Based Scaling}

For obvious reasons the transmitting node has more knowledge about the covariance matrix $\mathbf{C}$ to be transmitted. A more information efficient scaling should therefore be possible if the scaling is performed at the transmitting node.

Geometrically the PRE-EIG method described here is related to the previous case, see the illustration of Figure 5.2. We now want to find the point $x_{\lambda}$ since an axis-aligned ellipse intersecting this point will tightly enclose the ellipse of $\mathbf{C}$. This is done as follows. Transforming $\mathbf{C}$ and $\mathbf{D}$ by $\mathbf{T}=\mathbf{D}^{-\frac{1}{2}}$ yields

$$
\mathrm{TCT}^{\top}=\mathbf{Q}, \quad \mathrm{TDT}^{\top}=\mathbf{I},
$$

where $\mathbf{Q}$ is a correlation matrix. We seek a scaling factor $c_{\lambda}$ such that $c_{\lambda} \mathbf{I}$ tightly encloses $\mathbf{Q}$. This is equivalent to finding the largest eigenvalue $\lambda_{\max }^{\mathbf{Q}}$ of $\mathbf{Q}$ since if the vertex $\lambda_{\max }^{\mathbf{Q}} \mathbf{u}_{\max }$, where $\mathbf{u}_{\max }$ is the eigenvector corresponding to $\lambda_{\max }^{\mathbf{Q}}$, is enclosed by the sphere $\mathcal{E}\left(\mathbf{0}, c_{\lambda} \mathbf{I}\right)$ then

$$
\mathcal{E}(\mathbf{0}, \mathbf{Q}) \subseteq \mathcal{E}\left(\mathbf{0}, c_{\lambda} \mathbf{I}\right)
$$

i.e., $c_{\lambda}=\lambda_{\max }^{\mathrm{Q}}$ should be used. Transforming the point given by $\lambda_{\max }^{\mathbf{Q}} \mathbf{u}_{\max }$ using $\mathbf{T}^{-1}$ yields the point $\mathrm{x}_{\lambda}$.

The PRE-EIG method hence uses the conservative bound $\overline{\mathbf{D}}=c_{\lambda} \mathbf{D}=\lambda_{\max }^{\mathbf{Q}} \mathbf{D}$ meaning that $\hat{\mathbf{z}}$ and $\overline{\mathbf{D}}$ are transmitted to other nodes. In Theorem 5.2 it is shown that PRE-EIG preserves conservativeness. 


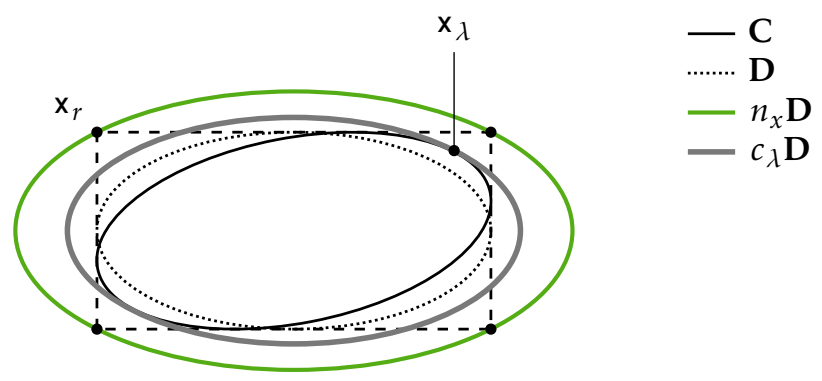

Figure 5.2: The axis-aligned ellipse within the rectangle, D, is the DCA of C.

Theorem 5.2. Let $\mathbf{C}$ be a covariance matrix and $\mathbf{D} \geq \mathbf{0}$ the diagonal matrix given by

$$
[\mathbf{D}]_{i j}= \begin{cases}{[\mathbf{C}]_{i j},} & \text { if } i=j, \\ 0, & \text { if } i \neq j .\end{cases}
$$

Then $c_{\lambda} \mathbf{D} \geq \mathbf{C}$ where $c_{\lambda}$ is the largest eigenvalue of the correlation matrix $\mathbf{Q}=$ $\mathbf{D}^{-\frac{1}{2}} \mathbf{C D}^{-\frac{1}{2}}$.

Proof: Assume $c \mathbf{D}-\mathbf{C} \geq \mathbf{0} \Longleftrightarrow c \mathbf{I}-\mathbf{Q} \geq \mathbf{0}$, where $\mathbf{Q}=\mathbf{D}^{-\frac{1}{2}} \mathbf{C D}^{-\frac{1}{2}}$ and $c$ is a scalar. The inequality $c \mathbf{I}-\mathbf{Q} \geq \mathbf{0}$ is equivalent to

$$
\mathbf{y}^{\top}\left(c \mathbf{I}-\sum_{i} \lambda_{i} \mathbf{u}_{i} \mathbf{u}_{i}^{\top}\right) \mathbf{y} \geq 0, \quad \forall \mathbf{y},
$$

where $\mathbf{Q}$ has been replaced by its eigendecomposition, i.e., $\mathbf{u}_{i}$ is the eigenvector corresponding to eigenvalue $\lambda_{i}$. Furthermore, $\mathbf{u}_{i}$ is normalized implying $\left\|\mathbf{y}^{\top} \mathbf{u}_{i}\right\| \leq$ $\|\mathbf{y}\|$. Without loss of generality assume $\|\mathbf{y}\|=1$ such that (5.8) reduces to

$$
c-\sum_{i} \lambda_{i} \mathbf{y}^{\top} \mathbf{u}_{i} \mathbf{u}_{i}^{\top} \mathbf{y} \geq 0, \quad \forall \mathbf{y},\|\mathbf{y}\|=1,
$$

which will be minimized by $\mathbf{y}=\mathbf{u}_{1}$ where $\mathbf{u}_{1}$ is the eigenvector corresponding to the maximum eigenvalue $\lambda_{1}$, i.e.,

$$
c-\sum_{i} \lambda_{i} \mathbf{u}_{1}^{\top} \mathbf{u}_{i} \mathbf{u}_{i}^{\top} \mathbf{u}_{1}=c-\lambda_{1} \geq 0 .
$$

By choosing $c=c_{\lambda}=\lambda_{1}$ we have $c_{\lambda} \mathbf{I}-\mathbf{Q} \geq \mathbf{0} \Longleftrightarrow c_{\lambda} \mathbf{D}-\mathbf{C} \geq \mathbf{0}$.

\subsubsection{Pre-Transmission Optimization-Based Scaling}

In PRE-OPT the scaling problem is formulated as an optimization problem. Let $\mathbf{c}=\left[\begin{array}{lll}c_{1} & \ldots & c_{n_{x}}\end{array}\right]$ denote the vector of scaling factors with $c_{i} \geq 1, \forall i$. Construct 
the matrix $\mathbf{D} \operatorname{diag}(\mathbf{c})$ and solve

$$
\begin{aligned}
\overline{\mathbf{D}}^{*}=\underset{\mathbf{c}}{\arg \min } & J(\mathbf{D} \operatorname{diag}(\mathbf{c})) \\
\text { subject to } & \mathbf{D} \operatorname{diag}(\mathbf{c})-\mathbf{C} \geq 0 .
\end{aligned}
$$

The matrix $\mathbf{D}$ diag(c) will generally be scaled non-uniformly. Solving for $\mathbf{D} \operatorname{diag}(\mathbf{c})$ yields the optimal diagonal matrix, given the matrix loss function $J(\cdot)$, that preserves a conservative estimate. The trace is here used as the matrix loss function.

By using $\bar{D}$ to denote the set of all diagonal matrices $\overline{\mathbf{D}}$ that satisfy $\overline{\mathbf{D}} \geq$ $\mathrm{C}^{\prime}, \forall \mathbf{C}^{\prime} \in \mathbb{C}$, the PRE-OPT method can equivalently be formulated as

$$
\overline{\mathbf{D}}^{*}=\underset{\overline{\mathbf{D}} \in \overline{\mathbf{D}}}{\arg \min } J(\overline{\mathbf{D}}) \text {. }
$$

\subsubsection{Pre-Transmission Diagonal-Dominance Scaling}

The proof of Theorem 5.1 utilizes diagonal dominance and its relationship to PSD and PD matrices which suggests the following argument. If a diagonal matrix $\mathbf{D}^{\prime}$ is constructed, where the $i$ th diagonal entry of $\mathbf{D}^{\prime}$ is the absolute sum of row $i$ of C, i.e.,

$$
\left[\mathbf{D}^{\prime}\right]_{i i}=\sum_{j=1}^{n_{x}}\left|[\mathbf{C}]_{i j}\right|,
$$

then $\mathbf{D}^{\prime}-\mathbf{C}$ automatically will be a symmetric diagonally dominant matrix and as such $\mathbf{D}^{\prime}-\mathbf{C}$ is PSD [19]. In the PRE-DOM method $\mathbf{D}^{\prime}$ is constructed according to (5.13) from which it follows $\mathbf{D}^{\prime}=\overline{\mathbf{D}} \geq \mathbf{C}$.

\subsection{Experimental Evaluation}

To evaluate the performance of the different methods and how conservative they are, a simulation study is made. The simulation study is based on a scenario where Kalman filters (KF, [27]) are used to filter measurements and in some cases to fuse estimates. See $[26,29]$ for a comprehensive introduction to Kalman filtering.

All of the previously described methods for preservation of conservative estimates will be evaluated. The following methods will also be included in the evaluation:

- CIF: Uses CI with access to the full covariance $\mathbf{C}$.

- LKF: Uses only information extracted by local sensors and a local KF.

- CKF: A centralized KF with access to the all information extracted by all sensors.

- NKF: Uses a naïve KF which assumes no cross-correlations between the estimates. In essence NKF is an application of the naïve fusion formula of (2.57). 


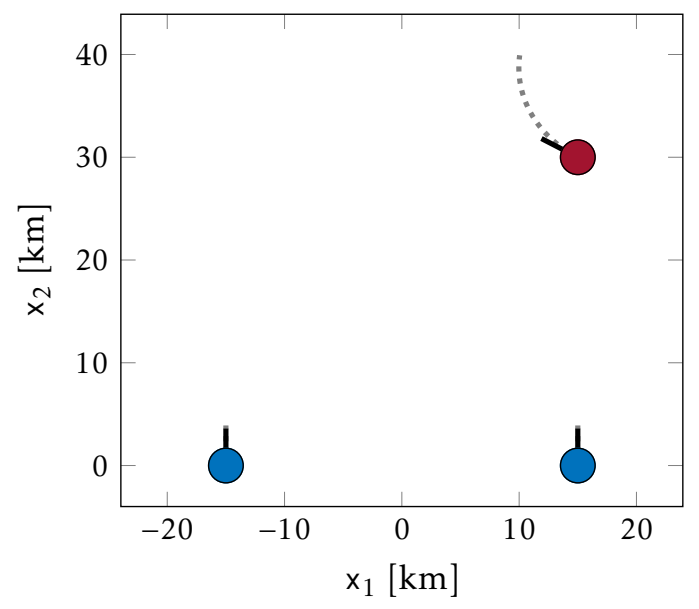

Figure 5.3: The simulated scenario. The target is given by the red node and two cooperating blue nodes approach the target from below.

\subsubsection{Simulation Scenario}

The (dynamic) scenario ${ }^{1}$ used for evaluation is illustrated in Figure 5.3. The target is represented by the red node in the upper part of the figure. The target follows the dotted trajectory. Two blue nodes approach the red node at straight parallel paths from below. Each of the blue nodes has a tracking sensor and they can also share information with each other via a datalink.

The simulations are performed in three dimensions such that the state $\mathbf{x}$ which comprises both position and velocity is six-dimensional. The true motions are constrained to a plane. All filters use a constant velocity model [34] where the process noise is tuned such that ANEES, see below, for CKF stabilizes at around 1. The range and bearing uncertainties of the tracking sensor are modeled as $\sigma_{r}=5000 \mathrm{~m}$ and $\sigma_{\theta}=0.5^{\circ}$, respectively.

The tracking sensor in each of the blue nodes generates new measurements of the target at a rate of $1 \mathrm{~Hz}$. An extended Kalman filter (EKF) is used to filter measurement [20]. The probability of detection is set to $100 \%$ and a clutter free environment is assumed. The update rate of the datalink is $1 \mathrm{~Hz}$.

A Monte Carlo (MC) simulation approach is considered with $2000 \mathrm{MC}$ runs for each method. The duration of each MC run is $20 \mathrm{~s}$. The simulation environment is implemented in MATLAB ${ }^{\circledR}$. The optimization problems introduced by POST-CI and PRE-OPT are solved using YALMIP [39].

\subsubsection{Evaluation Metrics}

The proposed methods are evaluated both w.r.t. their performance and their credibility. The performance of each method is evaluated using the root mean squared

\footnotetext{
${ }^{1}$ This scenario differs from the scenario in [12].
} 
error (RMSE). To have a meaningful physical interpretation of the RMSE the state is divided into its position and velocity components. The RMSE is calculated as the RMSE for specific time points over all MC runs.

The conservativeness property of the estimates for each method is evaluated by calculating the statistical measure normalized estimation error squared (NEES) defined as [4]

$$
\varepsilon(k)=(\mathbf{x}(k)-\hat{\mathbf{x}}(k \mid k))^{\top} \mathbf{P}^{-1}(k \mid k)(\mathbf{x}(k)-\hat{\mathbf{x}}(k \mid k)),
$$

where $k$ is a time index. The notation $k \mid k$ is used for filtered quantities. As $M$ number of MC simulations are performed multiple values of $\varepsilon_{i}(k)$ for each time point $k$ are generated. From these the average NEES (ANEES) at each time point $k$ can be calculated as [36]

$$
\bar{\varepsilon}(k)=\frac{1}{n_{x} M} \sum_{i=1}^{M} \varepsilon_{i}(k),
$$

i.e., ANEES is simply NEES averaged over the MC runs and normalized using the dimensionality $n_{x}$. A fusion rule providing ANEES significantly lower than 1 is regarded as conservative. A fusion rule providing ANEES significantly larger than 1 is regarded as too optimistic and non-conservative.

Confidence intervals for ANEES are derived in [35] and are calculated for $n_{x} M \gg 5$ according to

$$
\left[\left(c-p \sqrt{\frac{2}{9 n_{x} M}}\right)^{3},\left(c+p \sqrt{\frac{2}{9 n_{x} M}}\right)^{3}\right],
$$

where $c=1-2 /\left(9 n_{x} M\right)$ and $p$ depends on the confidence level, e.g., $p=1.96$ and $p=2.576$ for $95 \%$ and $99 \%$ confidence levels, respectively.

\subsubsection{Results and Discussion}

In Figure 5.4 ANEES over the MC simulations is presented. All methods, except NKF (and initially also CKF), lie below the solid line at ANEES equal to 1, hence indicating they provide conservative estimates throughout the simulation time. POST-CI, PRE-EIG, PRE-OPT and PRE-DOM are the most conservative methods giving an ANEES of about 0.3-0.5. Even though POST-SCALE produces the largest upscaled covariance of the scaling-oriented methods it is still slightly less conservative than POST-CI, PRE-EIG, PRE-OPT and PRE-DOM.

With the exception of NKF (see Figure 5.5) the position RMSE of all methods is bounded from below by CKF and from above by LKF. The optimistic NKF severely underestimates the covariance leading to estimates quickly begin to diverge. POST-SCALE performs the worst, presumably because the systematically up-scaling of $\mathbf{D}$ is followed by a substantial loss of information. Because of the relatively high update rate of the datalink, CIF quickly approaches the CKF. The velocity RMSE is given in Figure 5.6. Due to the constant speed of the tracked target the velocity RMSE converges relatively fast for all methods except NKF and LKF. 


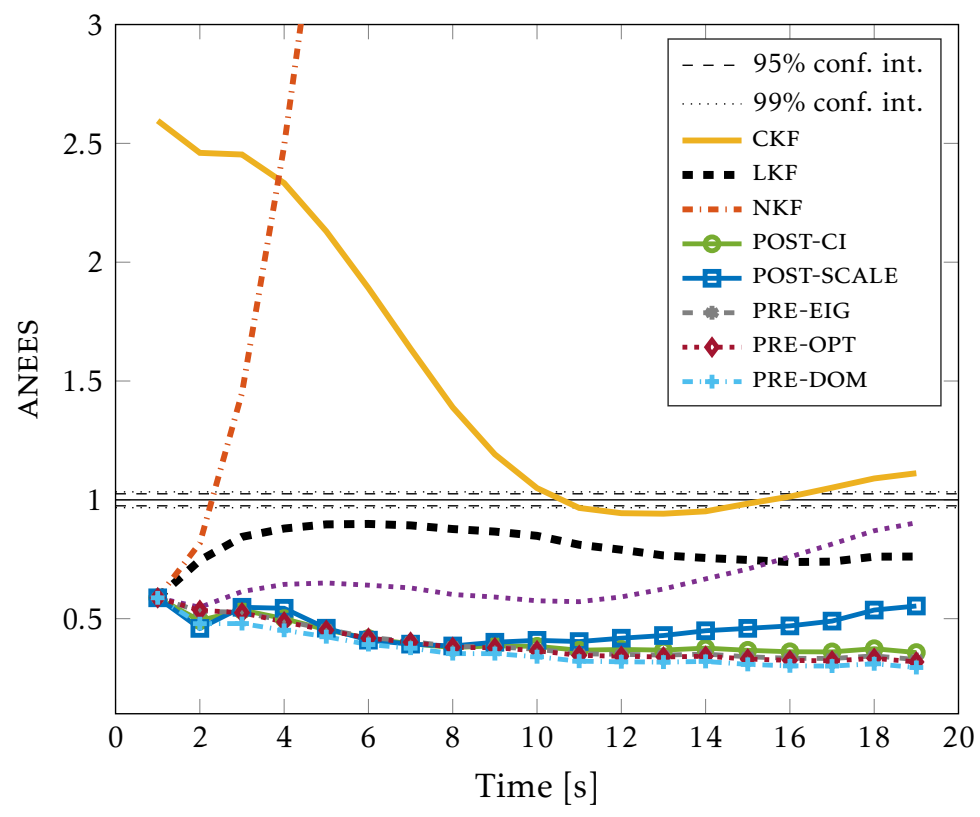

Figure 5.4: ANEES vs time elapsed since start of simulation. $95 \%$ and $99 \%$ confidence intervals for ANEES are also shown.

An interesting observation is that PRE-EIG, PRE-OPT and PRE-DOM provide approximately the same results, even though they are derived from different aspects. One explanation of PRE-EIG and PRE-OPT being very close in both RMSE and ANEES is that the PRE-OPT in fact finds the same scaled diagonal covariance matrix as do PRE-EIG. The performance of POST-CI being so close to PRE-EIG, PRE-OPT and PRE-DOM is beneficial for practical reasons; a platform operating in a network having a communication protocol constrained as assumed herein can preserve conservativeness of an exchanged estimate after transmission using POST-CI. One drawback is however that POST-CI involves solving a computational expensive optimization problem, which is also the case for PRE-OPT.

\subsubsection{Bandwidth Reduction}

Regarding the bandwidth utilization, to describe the full estimate $\hat{\mathbf{z}}$ and the full matrix $\mathbf{C}$ we need a number of $N_{p}^{F}=n_{x}+n_{x}\left(n_{x}+1\right) / 2=n_{x}\left(n_{x}+3\right) / 2$ parameters. The number of parameters required to describe $\hat{\mathbf{z}}$ and $\mathbf{D}$ is $N_{p}^{\text {DCA }}=2 n_{x}$. Table 5.1 provides examples of how the actual bandwidth demand differs between using the DCA and transmitting the full covariance. 


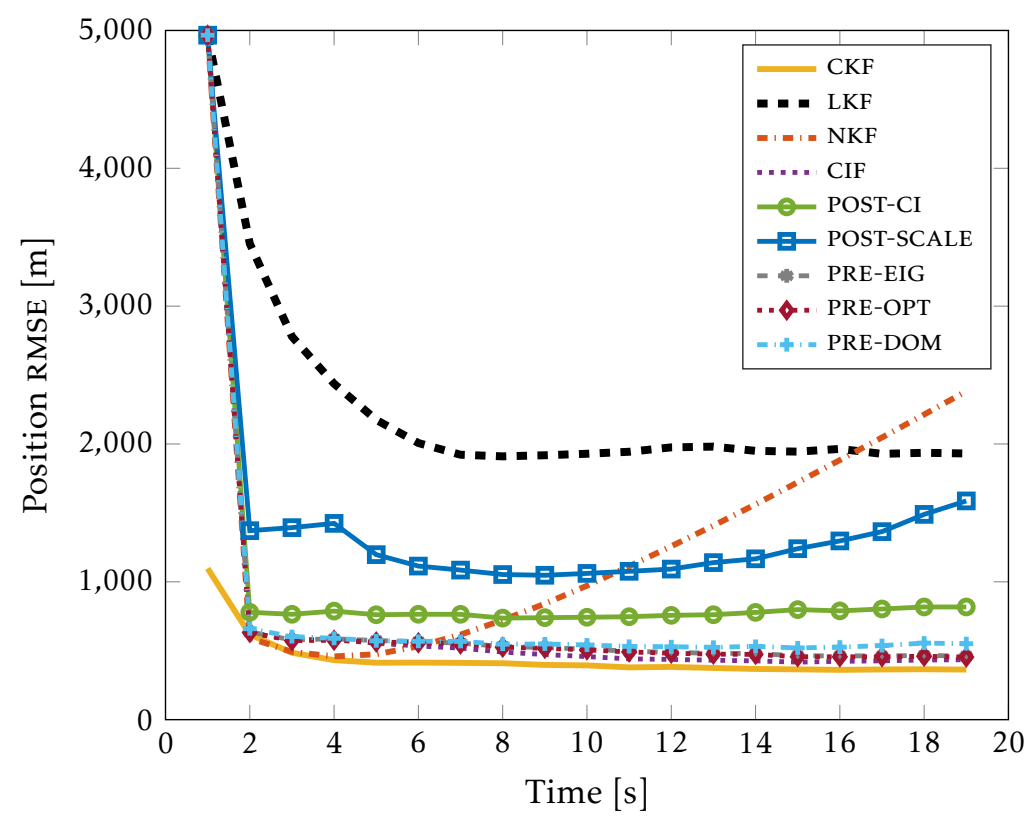

Figure 5.5: Position RMSE vs time elapsed since start of simulation.

Table 5.1: The number of parameters required when using the DCA compared to transmitting full covariance matrices.

\begin{tabular}{cccc}
\hline$n_{x}$ & $N_{p}^{F}$ & $N_{p}^{\text {DCA }}$ & Ratio $N_{p}^{\mathrm{DCA}} / N_{p}^{F}$ \\
\hline 3 & 9 & 6 & $66.7 \%$ \\
4 & 14 & 8 & $57.1 \%$ \\
6 & 27 & 12 & $44.4 \%$ \\
9 & 54 & 18 & $33.3 \%$ \\
\hline
\end{tabular}

\subsection{Summary}

In this chapter a number of methods suitable for decentralized estimation problems have been evaluated w.r.t. their performance and conservativeness when dealing with the communication constraint implied by the DCA.

The proposed methods have been used in conjunction with the CI algorithm. As CI requires that all input estimates are conservative to guarantee that the fused estimate is conservative, different methods for preservation of conservativeness have been developed. Of the proposed methods PRE-EIG, PRE-OPT and PRE-DOM perform equally well both w.r.t. the RMSE and the ANEES, and POST-CI shows almost as good performance. On the other hand, POST-SCALE shows a significantly worse performance w.r.t. RMSE, but with a ANEES closer to the optimal value 1. A remarkable result for practical applications is that POST-CI achieves almost 


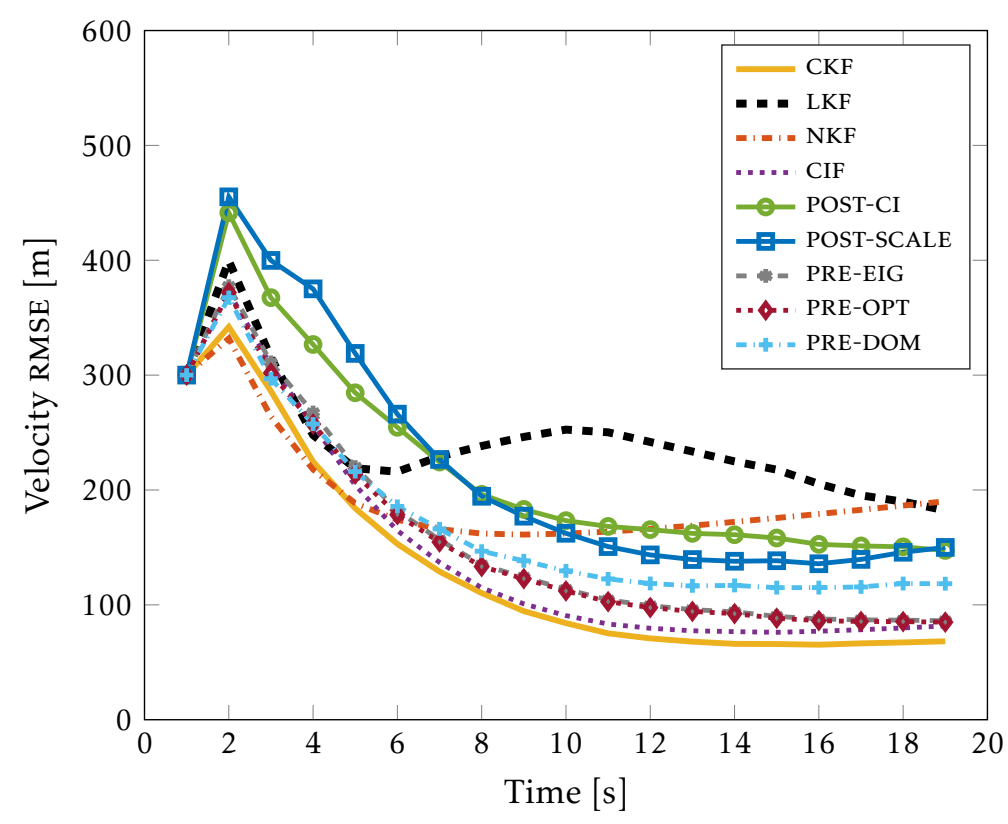

Figure 5.6: Velocity RMSE vs time elapsed since start of simulation.

as good performance as PRE-EIG, PRE-OPT and PRE-DOM, even though the conservativeness recovery is made post-transmission at the fusion node where full information about the original covariance $\mathbf{C}$ is not available.

Another way of looking at the results is from a bandwidth consumption perspective. If handled carefully, the DCA can considerably reduce the amount of data transmitted while still being able to preserve conservative estimates and to perform well in the RMSE sense. This is of importance in many real-world applications where sensor networks are required to act efficiently both in terms of communication bandwidth and performance. 


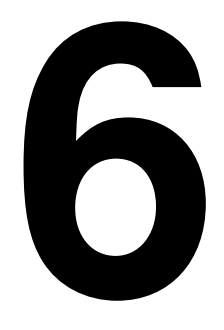

\section{Selective Information Extraction}

C

OMMUNICATION CONSTRAINTS must often be considered in decentralized estimation problems. It is simply not possible to transmit everything to everyone at every time instant. In the previous chapter the diagonal covariance approximation (DCA) is introduced as a way of reducing the bandwidth utilization of a datalink.

In this chapter a different but related concept is proposed. Instead of only transmitting an estimate and the diagonal entries of the corresponding covariance matrix, in this chapter an agent within a decentralized sensor network tries to decide and select the information which is useful for the remaining agents of the network. The methods derived for selecting information will be called information selectiagenton methods (ISM) [13].

The content of this chapter is published in:

Robin Forsling, Zoran Sjanic, Fredrik Gustafsson, and Gustaf Hendeby. Communication efficient decentralized track fusion using selective information extraction. In Proceedings of the 23rd IEEE International Conference on Information Fusion, Virtual Conference, July 2020.

Compared to [13] this chapter uses another notation.

\subsection{Problem Formulation}

Let a conservative partial estimate $\hat{\mathbf{z}}_{\imath}$ of a true state $\mathbf{x}$ be given where $\mathbf{C}_{\imath} \geq \operatorname{cov}\left(\hat{\mathbf{z}}_{\imath}\right)$ is the reported covariance of the estimate. The linear model $\hat{\mathbf{z}}_{\imath}=\mathbf{H}_{l} \mathbf{x}+\mathbf{e}_{\imath}$ is still assumed. Say now that we want to pick a subset of information from the information matrix $\mathbf{C}_{l}^{-1}$ which is to be exchanged with other agents. How should 
we decide which subset of $\mathbf{C}_{l}^{-1}$ is the most beneficial for the other agents? In this chapter we try to answer this question.

\subsubsection{Considered Estimation Problem}

We consider a decentralized sensor network where each local node is estimating $\mathbf{x}$ and where the estimates are exchanged between the different nodes. Roughly speaking, the estimation performance of such a setup depends on properties of the local estimates and the datalink.

Essentially, three types of estimation problems are implied by the problem formulation:

1. Local estimation: Obtaining estimates by filtering local measurements into a common coordinate frame used for representing the state of the object of interest.

2. Decentralized estimation: A decentralized data fusion problem in which the estimates received via datalink are merged with the local filter estimate. The cross-correlations between the fused estimates are assumed unknown.

3. Information selection: Selecting which information that is most valuable for the remaining nodes.

All estimates received via the datalink will be fused using CI. The choice of using CI for solving the decentralized data fusion problem is motivated since CI is able to guarantee conservatively fused estimates irrespective of the actual crosscorrelations given that the input estimates are conservative. See Section 4.1.2 for a recapitulation on $\mathrm{CI}$.

\subsubsection{Motivating Example}

As a motivating example, consider the information matrices $\mathbf{C}_{1}^{-1}$ and $\mathbf{C}_{2}^{-1}$ defined according to their information ellipses as illustrated in Figure 6.1. The gain of fusing $\mathbf{C}_{1}^{-1}$ with the full information $\mathbf{C}_{2}^{-1}$ is in this case not significantly higher than that of fusing with only the information along direction $\mathbf{v}_{1}$ in $\mathbf{C}_{2}$. Thus, the agent that has retrieved the information $\mathrm{C}_{2}^{-1}$ can, instead of transmitting the full information $\mathbf{C}_{2}^{-1}$, choose to transmit only the information along $\mathbf{v}_{1}$. In this way the bandwidth consumption can be reduced, while still generating high information fusion gains.

\subsubsection{Fusion of Arbitrary Projections}

Information selection is based on being able to fuse arbitrary projections. Therefore the following example is relevant. For now, the index $\imath$ will be dropped, i.e.,

$$
\hat{\mathbf{z}}_{l} \longrightarrow \hat{\mathbf{z}}, \quad \mathrm{C}_{l} \longrightarrow \mathrm{C} .
$$




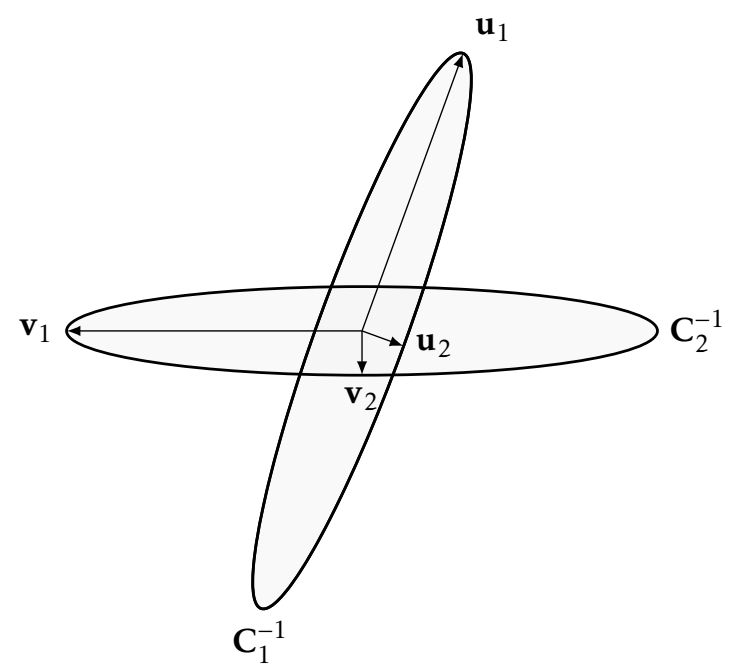

Figure 6.1: The ellipses of the information matrices $\mathbf{C}_{1}^{-1}$ and $\mathbf{C}_{2}^{-1}$. The principal axes of $\mathbf{C}_{1}^{-1}$ are given by $\mathbf{u}_{i}$ and the principal axes of $\mathbf{C}_{2}^{-1}$ are given by $\mathbf{v}_{i}$, where $i=1,2$.

Consider the partial estimate in space $\mathcal{Z}$ given by $\hat{\mathbf{z}}$ where $\mathbf{C}=\operatorname{cov}(\hat{\mathbf{z}})$. An estimate $\hat{\mathbf{z}}^{\prime}$, where $\mathbf{C}^{\prime}=\operatorname{cov}\left(\hat{\mathbf{z}}^{\prime}\right)$, in an arbitrary space $\mathcal{Z}^{\prime}$ can be constructed using the mapping $\mathbf{M}: \mathcal{Z} \longrightarrow \mathcal{Z}^{\prime}$ according to

$$
\hat{\mathbf{z}}^{\prime}=\mathbf{M} \hat{\mathbf{z}}, \quad \mathbf{C}^{\prime}=\mathrm{MCM}^{\top} .
$$

We limit ourselves to linear transformations and projections $\mathbf{M}$ with orthogonal basis vectors of unit length, i.e., the row vectors of $\mathbf{M}$ are orthonormal. The restriction to orthonormal projections is not necessary, but is useful as we will see later.

Now, the projections of $\hat{\mathbf{z}}$ and $\mathbf{C}$ along the direction defined by the unit vector $\mathbf{u}$ can be calculated by letting $\mathbf{M}=\mathbf{u}^{\top}$, i.e.,

$$
\hat{\mathbf{z}}_{\mathbf{u}}=\mathbf{u}^{\top} \hat{\mathbf{z}}, \quad \sigma_{\mathbf{u}}^{2}=\mathbf{u}^{\top} \mathbf{C u},
$$

where $\sigma_{\mathbf{u}}^{2}$ is the variance of the estimate $\hat{\mathbf{z}}$ in the direction defined by the vector u. Hence, arbitrary components can be extracted from both an estimate and the corresponding covariance by using projections. The fusion of such arbitrary projections is realized by using the projection as the (virtual) measurement model, i.e., the matrix $\mathbf{H}$.

\subsection{Information Selection Methods}

In this section three methods for selecting information are proposed. The information projections are contained as column vectors of unit length in $\mathbf{U}_{S}$. The 
number of columns in $\mathbf{U}_{S}$ is denoted by $n_{S}$. The information along the directions defined in $\mathbf{U}_{S}$ is contained as the diagonal entries in the diagonal matrix $\mathbf{D}_{S}$ of size $n_{S} \times n_{S}$. Thus, the partial information is fully described by $\mathbf{D}_{S}$ and $\mathbf{U}_{S}$, or equivalently by $\mathbf{U}_{S} \mathbf{D}_{S}$.

When selecting only a subset of projected information $\mathcal{I}_{S}=\mathbf{U}_{S} \mathbf{D}_{S}$, from an estimate $\hat{\mathbf{z}}$ with covariance $\mathbf{C}$, to be exchanged, the state estimate $\hat{\mathbf{z}}$ must be projected accordingly. The state estimate is projected using (6.3), i.e., $\hat{\mathbf{z}}_{S}=\mathbf{U}_{S}^{\top} \hat{\mathbf{z}}$. An equivalent approach would be to consider projected variances, but here we will focus on information simply because of its additive properties. Note, $\mathcal{I}_{S}$ defined as $\mathcal{I}_{S}=\mathbf{U}_{S} \mathbf{D}_{S}$ is not an information matrix in the strict sense, i.e., like the information matrix defined in Section 2.3.2. However, $\mathcal{I}_{S}$ contains all necessary parts for describing information within the scope of this chapter.

To minimize the bandwidth allocation a certain node can choose to transmit only $\left(\hat{\mathbf{z}}_{S}, \mathcal{I}_{S}\right)$. The node receiving $\left(\hat{\mathbf{z}}_{S}, \mathcal{I}_{S}\right)$ can derive $\mathbf{U}_{S}$ and $\mathbf{D}_{S}$ separately using the fact that each mutually orthogonal column $\mathbf{u}_{i}$ of $\mathbf{U}_{S}$ is of unit length. The considered projections are equivalent to the (virtual) measurement model given by $\mathbf{U}_{S}^{\top}$, meaning that $\mathbf{U}_{S}^{\top}$ corresponds to $\mathbf{H}$.

Three proposed ISM are introduced below, viz.,

- The largest eigenvalue (LEIG) method where only the largest information eigenvalues are exchanged.

- The transmitted information (TI) method where the exchanged information is selected based on previously transmitted information.

- The received information (RI) method where the exchanged information is selected based on previously received information.

\subsubsection{Aging Information}

So far in this chapter we have only considered static aspects of estimation, here in terms of fusion formulas. From now on, when the situation requires, we will use argument $k$ for quantities calculated at time $k$, with the argument $k \mid k$ being used for filtered quantities.

The TI method and the RI method are based upon maintaining a separate filtered covariance matrix $\hat{\mathbf{C}}(k \mid k)$, referred to as the reference covariance, which is used in the information selection process. $\hat{\mathbf{C}}(k \mid k)$ is only locally available. When selecting the currently most valuable information, old information should be forgotten. The aging of information can be done in different ways, but is here accomplished by predicting $\hat{\mathbf{C}}(k \mid k)$ according to

$$
\hat{\mathbf{C}}(k+1 \mid k)=\mathbf{F} \hat{\mathbf{C}}(k \mid k) \mathbf{F}^{\top}+\mathbf{Q},
$$

where $\mathbf{F}$ is the process model. The process noise covariance $\mathbf{Q}$ basically plays the role of a forgetting factor [29]. Any cross-correlations introduced by (6.4) are neglected. 


\subsubsection{Largest Eigenvalue Method}

The eigendecomposition of the PD matrix $\mathbf{C}$ is defined according to (2.9), i.e.,

$$
\mathbf{C}=\mathbf{V} \Sigma \mathbf{V}^{\top}=\sum_{i} \lambda_{i} \mathbf{v}_{i} \mathbf{v}_{i}^{\top}
$$

where $\Sigma$ is a diagonal matrix containing the $i$ th eigenvalue $\lambda_{i}$ of $\mathbf{C}$ at the $i$ th diagonal entry. $\mathbf{V}$ is an orthogonal matrix, containing the corresponding mutually orthogonal eigenvectors $\mathbf{v}_{i}$ as column vectors.

Since the information matrix $\mathbf{C}^{-1}$ is $\mathrm{PD}$ it can also be decomposed according to $(6.5)$, resulting in

$$
\mathbf{C}^{-1}=\left(\mathbf{V} \Sigma \mathbf{V}^{\top}\right)^{-1}=\mathbf{V} \Sigma^{-1} \mathbf{V}^{\top}=\sum_{i} \lambda_{i}^{-1} \mathbf{v}_{i} \mathbf{v}_{i}^{\top}
$$

The eigenvalues of $\mathbf{C}, \lambda_{i}$, are assumed to be sorted in ascending order implying that $\mathbf{C}^{-1}$ is the most informative along the $\mathbf{v}_{1}$ direction since $\lambda_{1}$ comprises the smallest uncertainty.

A trivial and straightforward selection of the subset $\mathcal{I}_{S}$ is to perform the eigendecomposition of $\mathbf{C}^{-1}$ and then select the $n_{S}$ largest eigenvalues. Hence, the LEIG method can be written compactly as

$$
\mathcal{I}_{S}=\left\{\left(\lambda_{i}^{-1}, \mathbf{v}_{i}\right)\right\}_{i=1}^{n_{S}} .
$$

\subsubsection{Transmitted Information Method}

A more sophisticated approach is to estimate what information the other nodes of the network lack. In the transmitted information (TI) method the introduced reference covariance $\hat{\mathbf{C}}$ is the result of fusing only information that has been transmitted from the own node.

The TI procedure is as follows: $\mathcal{I}_{S}$ which in turn is derived from the covariance $\mathbf{C}$ of a track is fused with $\hat{\mathbf{C}}$ where the fused result is given by $\hat{\mathbf{P}}$. That is, fusion of $\mathcal{I}_{S}$ and $\hat{\mathbf{C}}$ yields $\hat{\mathbf{P}}=\hat{\mathbf{P}}\left(\hat{\mathbf{C}}, \mathcal{I}_{S}\right)$. Denoting the spanning of all subsets $\mathcal{I}_{S}$ by $\mathcal{F}\left(\mathcal{I}_{S} ; n_{S}\right)$, where the size parameter $n_{S}$ has been explicitly included, the problem of selecting information can then be expressed as

$$
\underset{\mathcal{I}_{S} \in \mathcal{F}}{\arg \min } J\left(\hat{\mathbf{P}}\left(\hat{\mathbf{C}}, \mathcal{I}_{S}\right)\right),
$$

where $J(\cdot)$ is a matrix loss function which returns a scalar value. The fused covariance candidate $\hat{\mathbf{C}}$ is hence evaluated with respect to $J(\cdot)$ over the subsets $\mathcal{I}_{S} \in \mathcal{F}$. The information $\mathcal{I}_{S}$ minimizing $J(\cdot)$ is selected for transmission and is also fused with $\hat{\mathbf{C}}$.

TI will prefer information that has not yet been communicated. Schematics of TI is given in Figure 6.2. Initially when no information has been transmitted the TI method selects the largest eigenvalues. 


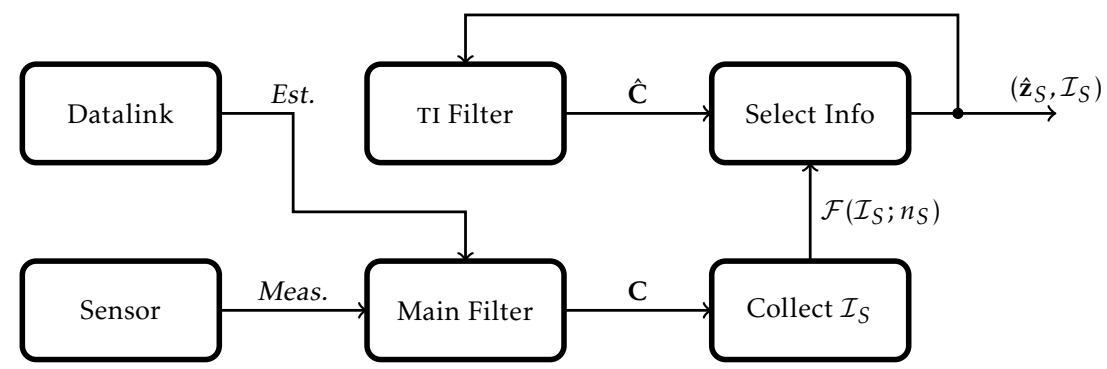

Figure 6.2: Schematics over the TI method. The main filter is updating the track estimate $\hat{\mathbf{z}}$ with own sensor measurements and datalink estimates. Information selected for transmission is fused with $\hat{\mathbf{C}}$ in the TI filter.

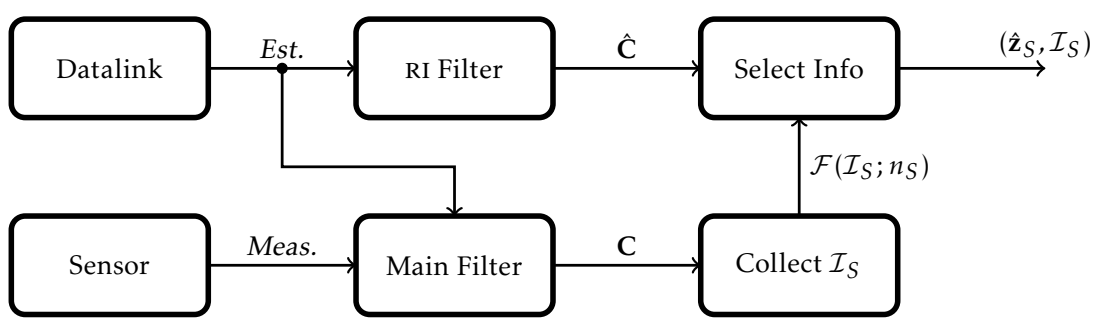

Figure 6.3: Schematics over the RI method. The main filter is updating the track estimate $\hat{\mathbf{z}}$ with own sensor measurements and datalink estimates. Information received via the datalink is fused with $\hat{\mathbf{C}}$ in the RI filter.

\subsubsection{Received Information Method}

The received information ( $\mathrm{RI}$ ) method is similar to the TI method given above but differs in how the reference covariance $\hat{\mathbf{C}}$ is calculated. In the RI method $\hat{\mathbf{C}}$ is fused only with information received from other nodes. RI will favour information not contained in the received information. RI is schematically illustrated in Figure 6.3.

\subsection{Experimental Evaluation}

In this section the simulation scenarios, the evaluation metrics and the results are presented and discussed. We will begin by mentioning the information projections that are considered in the experimental evaluation.

\subsubsection{Considered Information Projections}

So far we have considered information in arbitrary directions. Even though the methods proposed here hold for arbitrary projections of information we will, to simplify the following analysis, only deal with projections along the eigenvectors of the information matrix. 


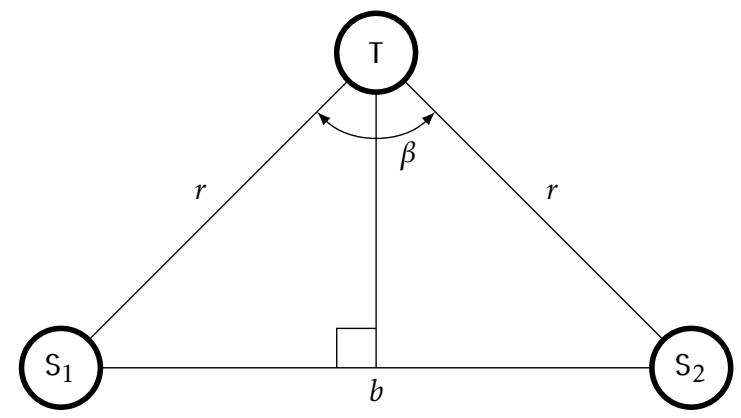

Figure 6.4: Two sensors, $\mathrm{S}_{1}$ and $\mathrm{S}_{2}$, observing a target $\mathrm{T}$ at range $r$. The baseline between the sensor nodes is $b$ which is related to $r$ and $\beta$.

The eigendecomposition, see (6.6), allows us to easily break down the information into orthogonal components. In essence, the matrix $\mathbf{V}$ in $\mathbf{C}^{-1}=\mathbf{V} \Sigma^{-1} \mathbf{V}^{\top}$ is a rotation matrix, rotating the diagonal and axis aligned matrix $\Sigma^{-1}$ into the frame that $\mathbf{C}^{-1}$ is represented in. For example, the information $\mathbf{C}_{\mathbf{v}_{i}}^{-1}$ can be calculated as

$$
\mathbf{C}_{\mathbf{v}_{i}}^{-1}=\mathbf{v}_{i}^{\top} \mathbf{C}^{-1} \mathbf{v}_{i}=\mathbf{v}_{i}^{\top} \mathbf{V} \Sigma^{-1} \mathbf{V}^{\top} \mathbf{v}_{i}=\lambda_{i}^{-1},
$$

where $\lambda_{i}$ is the $i$ th eigenvalue of $\mathbf{C}$ and where the orthogonality property of the eigenvectors has been used.

Using the eigendecomposition as the basis for information projections, reduces the optimization problem in (6.8) into a grid search among a finite set $\mathcal{F}$ consisting of combinations of information projections.

\subsubsection{Scenarios and Experimental Setup}

Our evaluation will be based on scenarios where the tracking sensors are highly accurate in bearing but the accuracy in range is poor, e.g., an infra-red search and track (IRST) system. The uncertainty in range for tracking filters deploying such sensors can be enhanced in a setup where the observed target is triangulated using two spatially separated sensors.

The estimation performance when triangulating depends on the geometry. A triangulation setup is illustrated in Figure 6.4. Two sensors, denoted $S_{1}$ and $S_{2}$, are observing a target T located at distance $r$ from each sensor. The baseline $b$ depends on both $r$ and the baseline angle $\beta$ according to

$$
b=2 r \sin \frac{\beta}{2} .
$$

The baseline angle $\beta$ will parametrize the geometry in the following.

We will evaluate the considered estimation problem on two extreme triangulation geometries:

Scenario 1. The baseline angle is approximately $90^{\circ}$. 
Scenario 2. The baseline angle is small but non-zero.

In addition to the proposed ISM a number of reference methods will be used in the evaluation. In total we have the following methods:

- LEIG: Largest eigenvalue method (Section 6.2.2).

- TI: Transmitted information method (Section 6.2.3).

- RI: Received information method (Section 6.2.4).

- CRLB: The Cramér-Rao lower bound (see Section 6.3.4 below).

- CKF: A centralized Kalman filter utilizing the unprocessed measurements from each sensor.

- LKF: A local Kalman filter having access only to local sensor data.

- CIF: A decentralized scheme deploying CI and the full filtered estimates from all sensing nodes.

Next, an analysis of the fusion gain dependency on the baseline angle is described. The purpose of the baseline angle analysis is to connect the two extreme geometries evaluated in Scenario 1 and 2.

\subsubsection{Baseline Angle Analysis}

To investigate how the fusion gain more generally depends on the geometry we introduce the covariance matrices $\mathbf{C}_{1}$ and $\mathbf{C}_{2}$ according to

$$
\begin{aligned}
& \mathbf{C}_{1}=\mathbf{T}(-\beta / 2) \mathbf{A}(a) \mathbf{T}(-\beta / 2)^{\top}, \\
& \mathbf{C}_{2}=\mathbf{T}(\beta / 2) \mathbf{A}(a) \mathbf{T}(\beta / 2)^{\top},
\end{aligned}
$$

respectively, where $\mathbf{A}$ and $\mathbf{T}$ are given by

$$
\begin{aligned}
\mathbf{A}(a) & =\left(\begin{array}{ll}
1 & 0 \\
0 & a
\end{array}\right) \\
\mathbf{T}(\alpha) & =\left(\begin{array}{cc}
\cos \alpha & -\sin \alpha \\
\sin \alpha & \cos \alpha
\end{array}\right) .
\end{aligned}
$$

The covariances $\mathbf{C}_{1}$ and $\mathbf{C}_{2}$ have the same eigenvalues as $\mathbf{A}$, but with the eigenvectors being rotated by $\mathbf{T}(-\beta / 2)$ and $\mathbf{T}(\beta / 2)$, respectively.

In the baseline angle analysis $\mathbf{C}_{1}^{-1}$ and $\mathbf{C}_{2}^{-1}$ are being fused using $\mathrm{CI}$ into $\mathbf{P}^{-1}$, with $a$ and $\beta$ varied. The weight parameters $\omega_{i}$ of $\mathrm{CI}$, see, e.g., the formula in (4.21), are optimized using the following loss functions: The determinant $\operatorname{det}(\mathbf{P})$, the trace $\operatorname{tr}(\mathbf{P})$, and the spectral norm $\lambda_{\max }(\mathbf{P})$. The following cases are studied: (1) $\mathbf{C}_{1}^{-1}$ is fused with full $\mathbf{C}_{2}^{-1}$, (2) $\mathbf{C}_{1}^{-1}$ is fused with only the largest eigenvalue of $\mathbf{C}_{2}^{-1}$, and (3) $\mathbf{C}_{1}^{-1}$ is fused with only the smallest eigenvalue of $\mathbf{C}_{2}^{-1}$. Figure 6.5 provides a summary of the baseline angle analysis. 

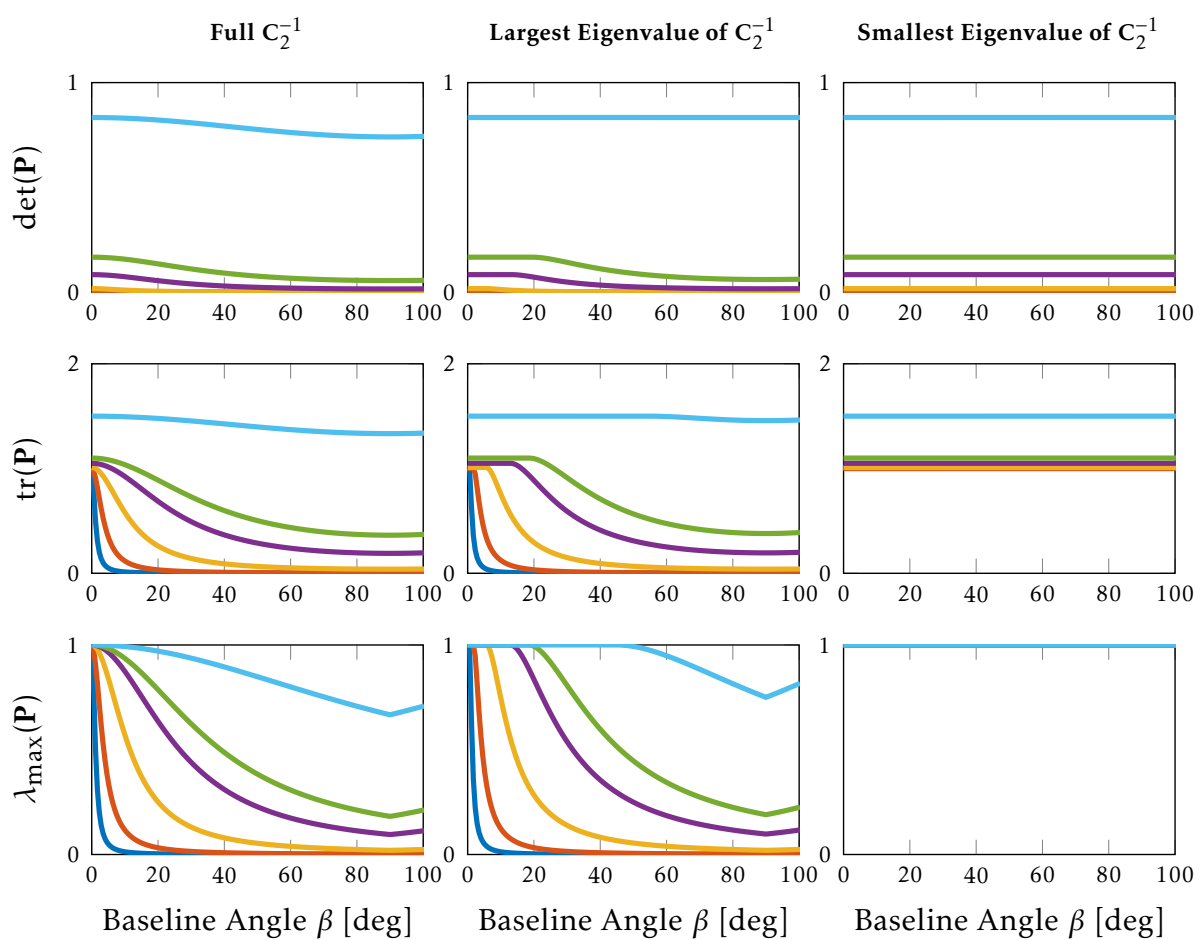

$\longrightarrow a=0.0001 \longrightarrow a=0.001-a=0.01 \longrightarrow a=0.05-a=0.1-a$

Figure 6.5: Analysis of how the fusion gain depends on the baseline angle $\beta$ for different norms of the covariance $\mathbf{P}$. The covariance $\mathbf{P}$ is the result from fusing the information $\mathbf{C}_{1}^{-1}$ with information given by full $\mathbf{C}_{2}^{-1}$, the largest eigenvalue of $\mathbf{C}_{2}^{-1}$ and the smallest eigenvalue of $\mathbf{C}_{2}^{-1} \cdot a^{-1}$ is the largest eigenvalue of each of $\mathbf{C}_{1}^{-1}$ and $\mathbf{C}_{2}^{-1}$. All curves are symmetric around $90^{\circ}$. 

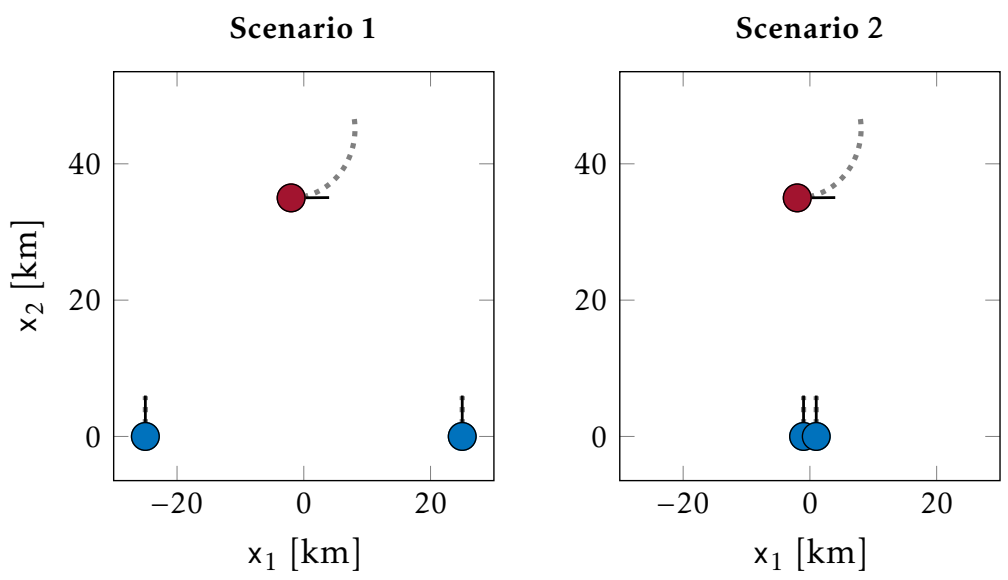

Figure 6.6: Simulation scenarios used in the experimental evaluation. The cooperating sensor nodes are colored blue, and the target, located above the sensor nodes in each case, is marked red. The black lines from a node indicate its initial orientation. Dotted lines represent the simulated trajectories.

\subsubsection{Evaluation Metrics}

The position component of the RMSE is used to measure the performance. The position RMSE for each time step over all MC runs is calculated, yielding a time series of position RMSE for each method. Conservativeness is evaluated using ANEES described in Section 5.3.2.

The parametric CRLB [62], $\mathbf{P}^{0}$, is calculated according to the following filter recursion

$$
\begin{aligned}
\mathbf{P}^{0}(k \mid k) & =\left(\left(\mathbf{P}^{0}(k \mid k-1)\right)^{-1}+\left(\mathbf{H}^{0}\right)^{\top} \mathbf{R}^{-1} \mathbf{H}^{0}\right)^{-1}, \\
\mathbf{P}^{0}(k+1 \mid k) & =\mathbf{F}^{0} \mathbf{P}^{0}(k \mid k)\left(\mathbf{F}^{0}\right)^{\top}+\mathbf{Q}^{0},
\end{aligned}
$$

where $\mathbf{F}^{0}$ is a model of the true dynamics, $\mathbf{H}^{0}=\left.\frac{d}{d \mathbf{x}^{\prime}} \mathbf{h}\left(\mathbf{x}^{\prime}\right)\right|_{\mathbf{x}^{\prime}=\mathbf{x}}$ is the true measurement model, $\mathbf{h}\left(\mathbf{x}^{\prime}\right)$ is the mapping from state coordinates to measurement coordinates, and $\mathbf{R}$ is the measurement covariance. The true process noise $\mathbf{Q}^{0}$ is derived from the true dynamics of the target. Time indices have been included in (6.13) since $\mathbf{P}^{0}$ is a filtered quantity.

\subsubsection{Simulation Specifications}

The two simulation scenarios are shown in Figure 6.6. In both scenarios there are two cooperating nodes sensing a target. In Scenario 1 the cooperating nodes are well separated, yielding a large baseline angle. In Scenario 2 the baseline angle is small. The simulations are performed in three dimensions with the dynamics being constrained to a plane. 

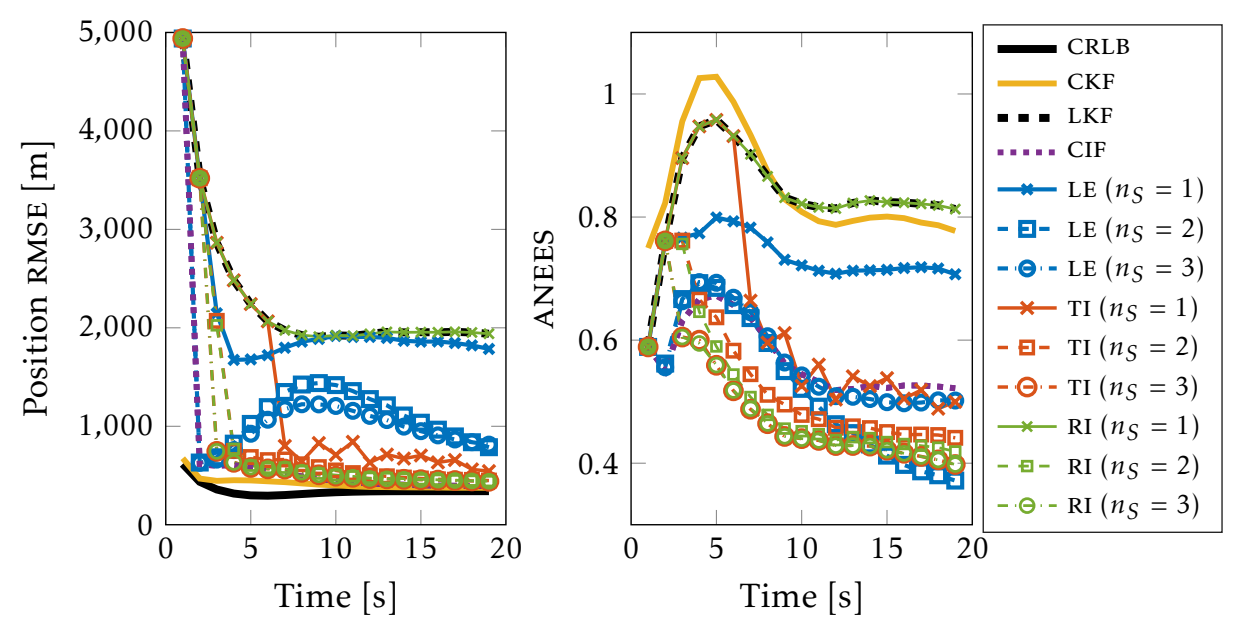

Figure 6.7: Scenario 1: Position RMSE to the left and ANEES to the right.

The cooperating nodes are synchronized and each tracking sensor generates new measurements at $1 \mathrm{~Hz}$. No clutter is simulated and the detection probability is $100 \%$. The measurement noise is white Gaussian noise, where the uncertainty in range and bearing is set to $5000 \mathrm{~m}$ and $0.5^{\circ}$, respectively. The measurements are filtered using an extended Kalman filter (EKF, [20]) and a constant velocity model [34] is used to describe the dynamics, i.e., $n_{x}=6$. The filter estimates are given in a Cartesian frame. The process noise is tuned such that the CKF initially achieves $\bar{\varepsilon}(k) \approx 1$ in Scenario 1 . The datalink runs at $1 / T_{\mathrm{DL}}=1 \mathrm{~Hz}$, where $T_{\mathrm{DL}}$ is the transmission period, and is scheduled to exchange filtered estimates immediately after each measurement update.

The simulation environment is implemented in MATLAB ${ }^{\circledR}$. $10000 \mathrm{MC}$ runs are performed for each method where the same noise realization is used in each case. The optimization problems implied by CI, TI and RI use the trace of the relevant covariance matrix as the loss function. The fused covariance candidate $\hat{\mathbf{P}}\left(\hat{\mathbf{C}}, \mathcal{I}_{S}\right)$ of TI and RI is generated using $(2.57 \mathrm{a})$. It should be noted that the results of using $(2.57 \mathrm{a})$ for optimizing the subset $\mathcal{I}_{S}$ are in general not the same as using CI for this optimization. However, the results would be the same as using CI constrained to $\omega=0.5$ since this is equivalent (up to a scaling factor) to $(2.57 \mathrm{a})$.

\subsubsection{Results and Discussion}

The results presented below are derived from only one of the two cooperating nodes. The results generated by the other cooperating node are approximately equivalent. This is true for both scenarios.

The simulation results, RMSE and ANEES, for Scenario 1 are given in Figure 6.7. $n_{S} \leq n_{x}=6$ is the number of eigenvalues used in the corresponding ISM. When $n_{S}=1$, only TI performs comparable to CIF, but with a slower transient. As suggested, increasing $n_{S}$ increases the performance. For TI and RI the performance 

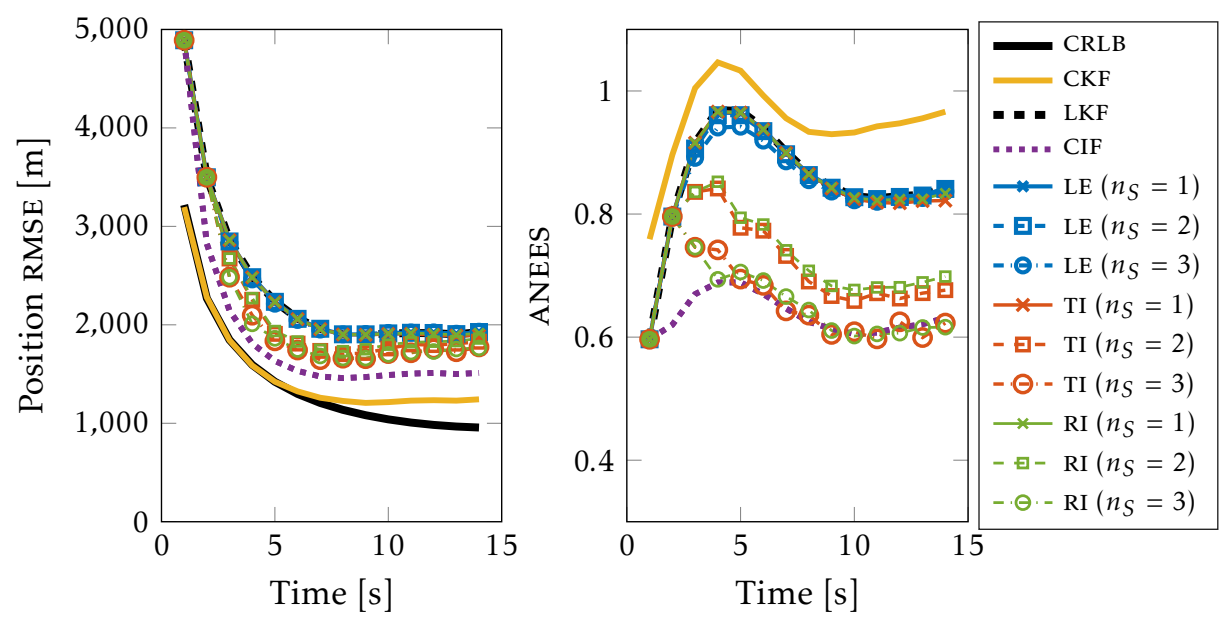

Figure 6.8: Scenario 2: Position RMSE to the left and ANEES to the right.

of CIF is reached when $n_{S} \geq 3$. Initially, LEIG performs well since the largest information eigenvalues will initially be the most useful ones, but there is a bump around $10 \mathrm{~s}$ for each LEIG curve. This bump is explained by the fact that the largest information eigenvalues are not always the most informative for the receiving node. In general, LEIG requires $n_{S}>3$ before the performance of CIF is reached. The RMSE for all methods are bounded from below by CKF and from above by LKF. CRLB is represented by the square root of the trace of the position part of $\mathbf{P}^{0}$.

For Scenario 2, RMSE and ANEES are given in Figure 6.8. The RMSE for all methods are bounded from below and above by CKF and LKF, respectively. For this geometry $n_{S}>3$ is required for all three ISM before the performance of CIF is approached.

Except for the initial peaks, all methods, both in Scenario 1 and 2, are conservative and quickly become even more conservative due to the accumulated process noise. The more eigenvalues exchanged typically implies a more conservatively fused estimate.

In Figure 6.9 and 6.10 the performance at $k_{e}=12 \mathrm{~s}$ is plotted for different $T_{\mathrm{DL}}$ for Scenario 1 and 2, respectively. Relatively speaking, the geometry provided by Scenario 1 leads to a higher sensitivity to $T_{\mathrm{DL}}$ than the geometry provided by Scenario 2.

The results from the baseline angle analysis are given in Figure 6.5. When full information $\mathbf{C}_{2}^{-1}$ is fused with $\mathbf{C}_{1}^{-1}$, the gain is strictly monotonically increasing when $\beta$ goes from $0^{\circ}$ to $90^{\circ}$, which is true for each norm and for each $a$. This is however not true for the case when only the largest eigenvalue of $\mathbf{C}_{2}^{-1}$ is fused with $\mathbf{C}_{1}^{-1}$, where there is no gain until a certain threshold angle $\beta_{t}$ is reached. The threshold $\beta_{t}$ seems to depend on $a$. As expected, no gain is achieved when fusing $\mathbf{C}_{1}^{-1}$ with the smallest eigenvalue of $\mathbf{C}_{2}^{-1}$. 


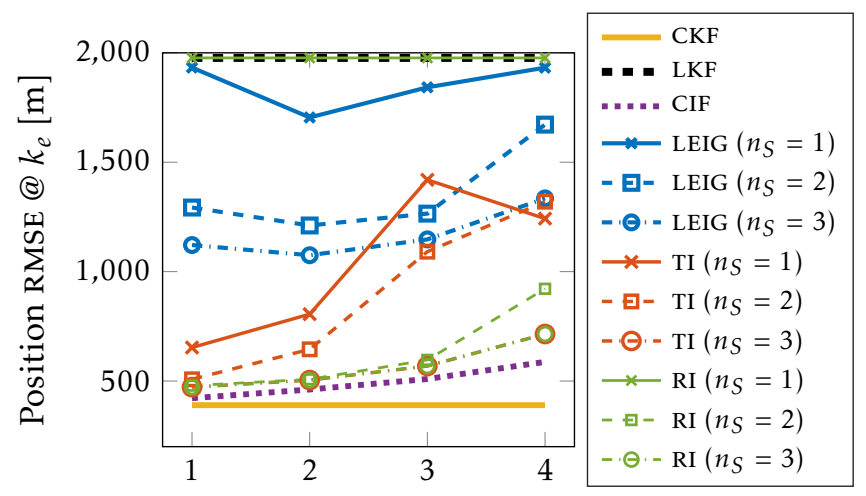

Datalink Transmission Period $T_{\mathrm{DL}}[\mathrm{s}]$

Figure 6.9: Scenario 1: Position RMSE evaluated at $k_{e}$ for different $T_{\mathrm{DL}}$.

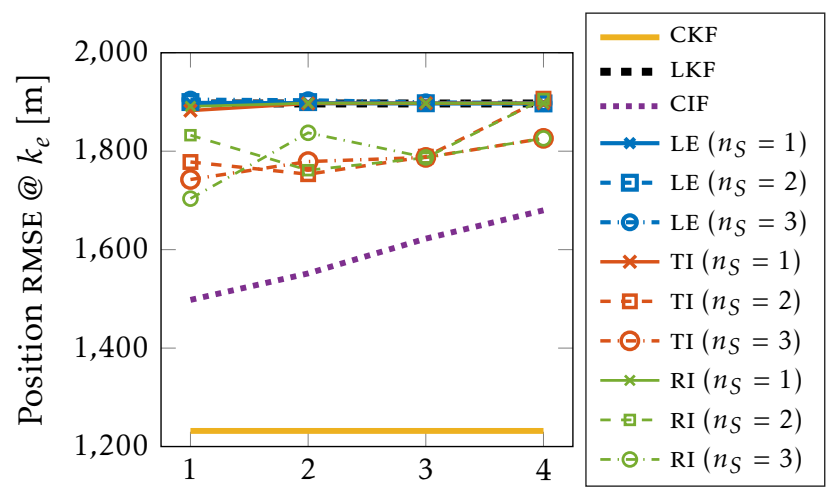

Datalink Transmission Period $T_{\mathrm{DL}}[\mathrm{s}]$

Figure 6.10: Scenario 2: Position RMSE evaluated at $k_{e}$ for different $T_{\mathrm{DL}}$.

The threshold $\beta_{t}$ reflects what is seen in the simulation results. LEIG performs poorly with a small baseline angle since no fusion gain is acquired at the receiving node when transmitting only the most informative projections.

\subsubsection{Bandwidth Reduction}

When considering the exchange of full estimates and full covariance matrices $n_{x}\left(n_{x}+3\right) / 2$ parameters must be transmitted. Using the proposed methods with $n_{S}=1$ only $n_{x}+1$ parameters are required. For arbitrary $n_{S}$ the number of parameters required in each transmission is calculated as $N_{p}=n_{S}+\sum_{i=1}^{n_{S}}\left(n_{x}-i+\right.$ $1)=n_{S}\left(3+2 n_{x}-n_{S}\right) / 2$ since we have assumed mutually orthogonal projections.

The bandwidth reduction is illustrated in Table 6.1 by calculating $N_{p}$ for different $n_{x}$ and $n_{S}$. 
Table 6.1: The number of parameters $N_{p}$ transmitted for different $n_{S}$ and $n_{x}$.

\begin{tabular}{cccc}
\hline$n_{S}$ & $N_{p}\left(n_{x}=4\right)$ & $N_{p}\left(n_{x}=6\right)$ & $N_{p}\left(n_{x}=9\right)$ \\
\hline 1 & 5 & 7 & 10 \\
2 & 9 & 13 & 19 \\
3 & 12 & 18 & 27 \\
4 & 14 & 22 & 34 \\
6 & - & 27 & 45 \\
9 & - & - & 54 \\
\hline
\end{tabular}

\subsection{Summary}

Three information selection methods (ISM) have been derived, suitable for a decentralized sensor network. The ISM try to select information projections based on what information is the most valuable for the remaining nodes. A comparison study of the ISM has been conducted, regarding their performance, measured as RMSE, and conservativeness, measured as ANEES. The evaluation was made using two triangulation setups.

The largest eigenvalue (LEIG) method suffers from its inability to prevent the same information projections from being repeatedly exchanged. Once the most informative projections have been transmitted, these will often become the most informative projections at the receiving node, which in turn will transmit the same information projections. This type of round trip inhibits complementary information from being extracted.

The transmitted information (TI) method and the received information (RI) method circumvent the previously discussed round trip issue by trying to maximize the fusion gain at the remaining nodes. Hence, not only the instantaneously most informative projections will be transmitted, but rather the projections that favours the considered fusion problem. This optimization strategy enables complementary information to be extracted in a larger extent.

Both TI and RI outperform the LEIG method in terms of RMSE. All ISM yield ANEES values lower than 1. A key feature of the suggested methods is that the bandwidth allocation of the communication link can be significantly reduced. It is also remarkable that none of the proposed ISM require any additional information than the communicated estimates themselves. 


\title{
Concluding Remarks
}

\begin{abstract}
A CONSERVATIVE approach to the decentralized estimation problem has been considered. The thesis and its contributions can roughly be divided into two major parts:

- Theoretical aspects of the conservative estimation problem. These were provided in Chapter 3 where the conservative linear unbiased estimator (CLUE) was defined, and perspectives and insights on the conservative linear unbiased estimation problem were given. In Chapter 4 established conservative fusion methods were investigated with respect to their properties.

- Practical applications of conservative estimation methods in decentralized sensor networks having communication constraints. The diagonal covariance approximation (DCA) was proposed in Chapter 5, and in Chapter 6 the communication constraints were handled using information selection methods (ISM).
\end{abstract}

\subsection{Summary and Conclusions}

One of the objectives presented in Chapter 1 was to propose an extension of linear estimation theory to the conservative linear estimation problem. In Chapter 3 this was done including examples that illustrated different aspects of conservative linear estimation.

The CLUE constitutes a realiable approach to the decentralized estimation problem where the error covariance of the input data is only partly known. In Chapter 3 it was seen that the conservative estimation problem is complicated but can be managed using a methodology adopted from (classical) linear estimation. When it comes to the optimal conservative estimator, the best CLUE and the 
restricted best CLUE were proposed, with the goal being to minimize the error covariance of the CLUE.

Several existing conservative fusion methods were described in Chapter 4, viz., covariance intersection (CI), inverse covariance intersection (ICI) and the largest ellipsoid (LE) method. The properties of each method were investigated. ICI and LE were further developed to be able to handle fusion of partial estimates.

The DCA proposed in Chapter 5 allows for the bandwidth utilization within a decentralized sensor network to be significantly reduced. Conservative estimates could be preserved while only transmitting the diagonal entries of an error covariance, i.e., essentially a vector.

In Chapter 6 the concept of ISM was introduced as an alternative to the DCA. Using the proposed ISM the information required by other agents of the decentralized sensor network could be selected such that only useful information were exchanged. Similar to the DCA approach, the ISM approach allows for the bandwidth utilization to be significantly reduced.

From a more general point-of-view the applications in Chapter 5 and 6 provide useful insights when it comes to conservative information extraction in general. Both applications illustrated that conservative information extraction generally differs a lot from its the classical counterpart when it comes to the estimation gain. One can say that in conservative estimation only complementary information yield any estimation gain since without complementary information it becomes impossible to distinguish new information that can be utilized in a conservative way.

\subsection{Future Work}

This thesis has opened up for future work within a variety of problem formulations, both theoretical and practical applications, but also combinations thereof.

The theoretical aspects and foundation of conservative linear unbiased estimation can be further developed. An interesting subject is optimality of the CLUE. In this work most focus has been on the restricted best CLUE, but the more general best CLUE should be given a deeper analysis. It might even be the case that the best CLUE and the restricted best CLUE turn out to be the same-at least for a subset of problems.

In Chapter 4 each conservative fusion method described was motivated from an underlying cross-correlation structure. Some of these cross-correlations structures might be hard to realize in practical problems in the strict sense. Hence, for practical problems it presumably is more relevant to know when the crosscorrelation structures are satisfied approximately and what approximately here really means for the solution.

Even though the LE method can only be ensured to be conservative for a rather restricted subset of problems, it is an interesting method from a theoretical pointof-view. Extending LE to handle multiple estimates based solely on an optimization formulation should be possible.

The practical applications in this work were based on using CI as the fusion 
rule for combining estimates. Both ICI and LE should be considered to replace CI. The performance evaluation should also be made by comparing the error covariance of the estimates, e.g., by looking at the trace of the error covariance.

No data association ambiguity and only a single target were considered in this scope. The DCA and ISM should be evaluated on multi-target problems where the data association problem cannot be solved without ambiguity. It should also be possible to combine DCA and ISM.

All practical applications considered were based on simulation studies. Testing the proposed methods on real data and on real-time systems would be useful in further development of the methods. 


\section{Bibliography}

[1] Jiri Ajgl and Ondrej Straka. Inverse covariance intersection fusion of multiple estimates. In Proceedings of the 23rd IEEE International Conference on Information Fusion, Virtual Conference, July 2020.

[2] Muhammad Abu Bakr and Sukhan Lee. Comparative analysis of ellipsoidal methods for distributed data fusion. In Proceedings of the 12th International Conference on Ubiquitous Information Management and Communication, Langkawi, Malaysia, January 2018.

[3] Yaakov Bar-Shalom and Leon Campo. The effect of the common process noise on the two-sensor fused-track covariance. IEEE Transactions on Aerospace and Electronic Systems, 22(6):803-805, November 1986.

[4] Yaakov Bar-Shalom, X.R. Li, and T. Kirubarajan. Estimation with Applications to Tracking and Navigation: Theory Algorithms and Software. John Wiley \& Sons, Ltd, New York, NY, USA, 2004.

[5] Abder R. Benaskeur. Consistent fusion of correlated data sources. In Proceedings of the 28th Annual Conference of the IEEE Industrial Electronics Society, pages 2652-2656, Sevilla, Spain, November 2002.

[6] Samuel S. Blackman and Robert Popoli. Design and analysis of modern tracking systems. Artech House, Norwood, MA, USA, 1999.

[7] Stephen Boyd and Lieven Vandenberghe. Convex Optimization. Cambridge University Press, New York, NY, USA, 2004.

[8] Federico Castanedo. A review of data fusion techniques. The Scientific World Journal, 2013:1-19, 2013.

[9] Lingji Chen, Pablo Arambel, and Raman Mehra. Estimation under unknown correlation: Covariance intersection revisited. IEEE Transactions on Automatic Control, 47:1879-1882, November 2002.

[10] Lingji Chen, Pablo Arambel, and Raman Mehra. Fusion under unknown correlation: Covariance intersection as a special case. In Proceedings of the 
5th IEEE International Conference on Information Fusion, Annapolis, MD, USA, July 2002.

[11] Chee-Yee Chong, Shozo Mori, and Kuo-Chu Chang. Distributed multitarget multisensor tracking. In Yaakov Bar-Shalom, editor, MultitargetMultisensor Tracking: Advanced Applications, chapter 8. Artech House, Norwood, MA, USA, 1990.

[12] Robin Forsling, Zoran Sjanic, Fredrik Gustafsson, and Gustaf Hendeby. Consistent distributed track fusion under communication constraints. In Proceedings of the 22nd IEEE International Conference on Information Fusion, Ottawa, Canada, July 2019.

[13] Robin Forsling, Zoran Sjanic, Fredrik Gustafsson, and Gustaf Hendeby. Communication efficient decentralized track fusion using selective information extraction. In Proceedings of the 23rd IEEE International Conference on Information Fusion, Virtual Conference, July 2020.

[14] Dietrich Fränken and Andreas Hüpper. Improved fast covariance intersection for distributed data fusion. In Proceedings of the 8th IEEE International Conference on Information Fusion, Philadelphia, PA, USA, July 2005.

[15] Carl Friedrich Gauss. Theoria motus corporum coelestium in sectionibus conicis solem ambientium. 1809.

[16] Felix Govaers and Wolfgang Koch. An exact solution to track-to-track-fusion at arbitrary communication rates. IEEE Transactions on Aerospace and Electronic Systems, 48(3):2718-2729, 2012.

[17] Stewart H. Grime and Hugh F. Durrant-Whyte. Data fusion in decentralized sensor networks. Control Engineering Practice, 2(5):849 - 863, 1994.

[18] Fredrik Gustafsson. Statistical Sensor Fusion. Studentlitteratur, Lund, Sweden, 2018.

[19] Roger A. Horn and Charles R. Johnson. Matrix Analysis. Cambridge University Press, New York, NY, USA, 2012.

[20] Andrew H. Jazwinski. Stochastic processes and filtering theory. Academic Press, New York, NY, USA, 1970.

[21] Fritz John. Extremum problems with inequalities as subsidiary conditions. In Jürgen Moser, editor, Fritz John, Collected Papers, pages 543-560. Birkhäuser, Basel, Switzerland, 1985. First published in 1948.

[22] Simon J. Julier and Jeffrey K. Uhlmann. A non-divergent estimation algorithm in the presence of unknown correlations. In Proceedings of the 1997 American Control Conference, pages 2369-2373, Albuquerque, NM, USA, June 1997. 
[23] Simon J. Julier and Jeffrey K. Uhlmann. General decentralized data fusion with covariance intersection. In Martin Liggins, David Hall, and James Llinas, editors, Handbook of Multisensor Data Fusion: Theory and Practice, chapter 14. CRC Press, Boca Raton, FL, USA, 2009.

[24] William Kahan. Circumscribing an ellipsoid about the intersection of two ellipsoids. Canadian Mathematical Bulletin, 11(3):437-441, 1968.

[25] William Kahan. Circumscribing an ellipsoid about the intersection of two ellipsoids. 2006.

[26] Thomas Kailath, Ali H. Sayed, and Babak Hassibi. Linear Estimation. Prentice Hall, Upper Saddle River, NJ, USA, 2000.

[27] Rudolf E. Kalman. A new approach to linear filtering and prediction problems. Transactions of the ASME-Journal of Basic Engineering, 82(Series D): 35-45, 1960.

[28] Lance M. Kaplan, William D. Blair, and Yaakov Bar-Shalom. Simulations studies of multisensor track association and fusion methods. In Proceedings of the IEEE Aerospace Conference, Big Sky, MT, USA, March 2006.

[29] Steven M. Kay. Fundamentals of Statistical Signal Processing: Estimation theory. Fundamentals of Statistical Signal Processing. Prentice Hall, Upper Saddle River, NJ, USA, 1993.

[30] Naoto Kimura and Shahram Latifi. A survey on data compression in wireless sensor networks. In Proceedings of the IEEE International Conference on Information Technology: Coding and Computing, volume 2, pages 8-13, Las Vegas, NV, USA, April 2005.

[31] Alexander B. Kurzhanski and István Vályi. Ellipsoidal Calculus for Estimation and Control. Birkhäuser, Boston, MA, USA, 1996.

[32] Adrien-Marie Legendre. Nouvelles Méthods pour la Détermination des Orbites de Cométes Courcier. Paris, France, 1805.

[33] Erich L. Lehmann and George Casella. Theory of Point Estimation. SpringerVerlag, New York, NY, USA, 2nd edition, 1998.

[34] X. Rong Li and Vesselin P. Jilkov. Survey of maneuvering target tracking. Part I. Dynamic models. IEEE Transactions on Aerospace and Electronic Systems, 39(4):1333-1364, 2003.

[35] X. Rong Li, Zhanlue Zhao, and Vesselin P. Jilkov. Practical measures and test for credibility of an estimator. In Proceedings of Workshop on Estimation, Tracking and Fusion: A tribute to Yaakov Bar-Shalom, Monterey, CA, USA, May 2001.

[36] X. Rong Li, Zhanlue Zhao, and Vesselin P. Jilkov. Estimator's credibility and its measures. In Proceedings of the 15th Triennial IFAC World Congress, Barcelona, Spain, July 2002. 
[37] Martin E. Liggins and Kuo-Chu Chang. Distributed fusion architectures, algorithms, and performance within a network-centric architecture. In Martin Liggins, David Hall, and James Llinas, editors, Handbook of Multisensor Data Fusion: Theory and Practice, chapter 17. CRC Press, Boca Raton, FL, USA, 2009.

[38] Lennart Ljung. System Identification: Theory for the User. Prentice Hall, Upper Saddle River, NJ, USA, 2nd edition, 1999.

[39] Johan Löfberg. Yalmip: A toolbox for modeling and optimization in MATLAB. In Proceedings of the CACSD Conference, volume 3, Taipei, Taiwan, September 2004.

[40] Samuel McLaughlin, Rob Evans, and Vikram Krishnamurthy. Data incest removal in a survivable estimation fusion architecture. In Proceedings of the 6th IEEE International Conference on Information Fusion, Cairns, Queensland, Australia, July 2003.

[41] Samuel McLaughlin, Vikram Krishnamurthy, and Subhash Challa. Managing data incest in a distributed sensor network. In Proceedings of the IEEE International Conference on Acoustics, Speech, and Signal Processing, April 2003.

[42] Shozo Mori, Kuo-Chu Chang, and Chee-Yee Chong. Distributed tracking in distributed sensor networks. In Proceedings of the 1986 American Control Conference, Seattle, WA, USA, June 1986.

[43] Arthur G. O. Mutambara. Decentralized Estimation and Control for Multisensor Systems. CRC Press, Boca Raton, FL, USA, 1998.

[44] Arthur G. O. Mutambara and Hugh F. Durrant-Whyte. Nonlinear information space: A practical basis for decentralization. In Proceedings of SPIE Sensor Fusion VII, volume 2355, pages 97-105, October 1994.

[45] Wolfgang Niehsen. Information fusion based on fast covariance intersection filtering. In Proceedings of the 5th IEEE International Conference on Information Fusion, pages 901-904, Annapolis, MD, USA, July 2002.

[46] Benjamin Noack, Daniel Lyons, Matthias Nagel, and Uwe D. Hanebeck. Nonlinear information filtering for distributed multisensor data fusion. In Proceedings of the 2011 American Control Conference, San Fransisco, CA, USA, June 2011.

[47] Benjamin Noack, Joris Sijs, and Uwe D. Hanebeck. Algebraic analysis of data fusion with ellipsoidal intersection. In Proceedings of the IEEE International Conference on Multisensor Fusion, pages 365-370, Baden-Baden, Germany, September 2016.

[48] Benjamin Noack, Joris Sijs, and Uwe D. Hanebeck. Inverse covariance intersection: New insights and properties. In Proceedings of the 20th IEEE International Conference on Information Fusion, Xi'an, China, July 2017. 
[49] Benjamin Noack, Joris Sijs, Marc Reinhardt, and Uwe D. Hanebeck. Decentralized data fusion with inverse covariance intersection. Automatica, 79: 35-41, May 2017.

[50] Reza Olfati-Saber. Distributed Kalman filter with embedded consensus filters. In Proceedings of the 44th IEEE Conference Decision and Control, Sevilla, Spain, December 2005.

[51] Lucy Y. Pao and Mike Kalandros. Algorithms for a class of distributed architecture tracking. In Proceedings of the 1997 American Control Conference, Albuquerque, NM, USA, June 1997.

[52] Susanne Radtke, Benjamin Noack, Uwe D. Hanebeck, and Ondrej Straka. Reconstruction of cross-correlations with constant number of deterministic samples. In Proceedings of the 21th IEEE International Conference on Information Fusion, Cambridge, UK, July 2018.

[53] Susanne Radtke, Benjamin Noack, and Uwe D. Hanebeck. Fully decentralized estimation using square-root decompositions. In Proceedings of the 23rd IEEE International Conference on Information Fusion, Virtual Conference, July 2020.

[54] C. Radhakrishna Rao. Linear Statistical Inference and its Applications. Wiley, New York, NY, USA, 2nd edition, 2001.

[55] Marc Reinhardt, Benjamin Noack, Marcus Baum, and Uwe D. Hanebeck. Analysis of set-theoretic and stochastic models for fusion under unknown correlations. In Proceedings of the 14th IEEE International Conference on Information Fusion, Chicago, IL, USA, July 2011.

[56] Marc Reinhardt, Benjamin Noack, and Uwe D. Hanebeck. Closed-form optimization of covariance intersection for low-dimensional matrices. In Proceedings of the 15th IEEE International Conference on Information Fusion, pages 1891-1896, Singapore, July 2012.

[57] Marc Reinhardt, Benjamin Noack, and Uwe D. Hanebeck. The hypothesizing distributed kalman filter. In Proceedings of the IEEE International Conference on Multisensor Fusion, Hamburg, Germany, September 2012.

[58] Marc Reinhardt, Benjamin Noack, Pablo O. Arambel, and Uwe D. Hanebeck. Minimum covariance bounds for the fusion under unknown correlations. IEEE Signal Processing Letters, 22(9):1210-1214, 2015.

[59] Joris Sijs and Mircea Lazar. Empirical case-studies of state fusion via ellipsoidal intersection. In Proceedings of the 17th IEEE International Conference on Information Fusion, Salamanca, Spain, July 2014.

[60] Joris Sijs, Mircea Lazar, and P. P. J. van den Bosch. State fusion with unknown correlation: Ellipsoidal intersection. In Proceedings of the 2010 American Control Conference, pages 3992-3997, Baltimore, MD, USA, June 2010. 
[61] Jannik Steinbring, Benjamin Noack, Marc Reinhardt, and Uwe D. Hanebeck. Optimal sample-based fusion for distributed state estimation. In Proceedings of the 19th IEEE International Conference on Information Fusion, Heidelberg, Germany, July 2016.

[62] James H. Taylor. The Cramér-Rao estimation error lower bound computation for deterministic nonlinear systems. IEEE Transactions on Automatic Control, 24(2):343-344, April 1979.

[63] Paul Thompson, Eric Nettleton, and Hugh F. Durrant-Whyte. Decentralized data fusion: Formulation and algorithms. In David Hall, Chee-Yee Chong, James Llinas, and Martin Liggins, editors, Distributed Data Fusion for Network-Centric Operations, chapter 7. CRC Press, Boca Raton, FL, USA, 2012.

[64] Xin Tian and Yaakov Bar-Shalom. Exact algorithms for four track-to-track fusion configurations: All you wanted to know but were afraid to ask. In Proceedings of the 12th IEEE International Conference on Information Fusion, Seattle, WA, USA, July 2009.

[65] Xin Tian and Yaakov Bar-Shalom. On algorithms for asynchronous track-totrack fusion. In Proceedings of the 13th IEEE International Conference on Information Fusion, Edinburgh, Scotland, July 2010.

[66] Jeffrey K. Uhlmann. General data fusion for estimates with unknown cross covariances. In Proceedings of SPIE Aerosense Conference, volume 2755, pages 536-547, Orlando, FL, USA, April 1996.

[67] Pramod K. Varshney, Engin Masazade, Priyadip Ray, and Ruixin Niu. Distributed detection in wireless sensor networks. In David Hall, Chee-Yee Chong, James Llinas, and Martin Liggins, editors, Distributed Data Fusion for Network-Centric Operations, chapter 4. CRC Press, Boca Raton, FL, USA, 2012.

[68] Max A. Woodbury. Inverting modified matrices. Department of Statistics, Princeton University, Princeton, NJ, USA, 1950.

[69] Yan Zhou and Jianxun Li. Data fusion of unknown correlations using internal ellipsoidal approximation. In Proceedings of the 17th Triennial IFAC World Congress, pages 2856 - 2860, Seoul, Korea, July 2008. 


\section{Licentiate Theses \\ Division of Automatic Control \\ Linköping University}

P. Andersson: Adaptive Forgetting through Multiple Models and Adaptive Control of Car Dynamics. Thesis No. 15, 1983.

B. Wahlberg: On Model Simplification in System Identification. Thesis No. 47, 1985.

A. Isaksson: Identification of Time Varying Systems and Applications of System Identification to Signal Processing. Thesis No. 75, 1986.

G. Malmberg: A Study of Adaptive Control Missiles. Thesis No. 76, 1986.

S. Gunnarsson: On the Mean Square Error of Transfer Function Estimates with Applications to Control. Thesis No. 90, 1986.

M. Viberg: On the Adaptive Array Problem. Thesis No. 117, 1987.

K. Ståhl: On the Frequency Domain Analysis of Nonlinear Systems. Thesis No. 137, 1988.

A. Skeppstedt: Construction of Composite Models from Large Data-Sets. Thesis No. 149, 1988.

P. A. J. Nagy: MaMiS: A Programming Environment for Numeric/Symbolic Data Processing. Thesis No. 153, 1988.

K. Forsman: Applications of Constructive Algebra to Control Problems. Thesis No. 231, 1990.

I. Klein: Planning for a Class of Sequential Control Problems. Thesis No. 234, 1990.

F. Gustafsson: Optimal Segmentation of Linear Regression Parameters. Thesis No. 246, 1990.

H. Hjalmarsson: On Estimation of Model Quality in System Identification. Thesis No. 251, 1990.

S. Andersson: Sensor Array Processing; Application to Mobile Communication Systems and Dimension Reduction. Thesis No. 255, 1990.

K. Wang Chen: Observability and Invertibility of Nonlinear Systems: A Differential Algebraic Approach. Thesis No. 282, 1991.

J. Sjöberg: Regularization Issues in Neural Network Models of Dynamical Systems. Thesis No. 366, 1993.

P. Pucar: Segmentation of Laser Range Radar Images Using Hidden Markov Field Models. Thesis No. 403, 1993.

H. Fortell: Volterra and Algebraic Approaches to the Zero Dynamics. Thesis No. 438, 1994.

T. McKelvey: On State-Space Models in System Identification. Thesis No. 447, 1994.

T. Andersson: Concepts and Algorithms for Non-Linear System Identifiability. Thesis No. 448, 1994.

P. Lindskog: Algorithms and Tools for System Identification Using Prior Knowledge. Thesis No. 456, 1994.

J. Plantin: Algebraic Methods for Verification and Control of Discrete Event Dynamic Systems. Thesis No. 501, 1995.

J. Gunnarsson: On Modeling of Discrete Event Dynamic Systems, Using Symbolic Algebraic Methods. Thesis No. 502, 1995.

A. Ericsson: Fast Power Control to Counteract Rayleigh Fading in Cellular Radio Systems. Thesis No. 527, 1995.

M. Jirstrand: Algebraic Methods for Modeling and Design in Control. Thesis No. 540, 1996.

K. Edström: Simulation of Mode Switching Systems Using Switched Bond Graphs. Thesis No. 586, 1996. 
J. Palmqvist: On Integrity Monitoring of Integrated Navigation Systems. Thesis No. 600, 1997.

A. Stenman: Just-in-Time Models with Applications to Dynamical Systems. Thesis No. 601, 1997.

M. Andersson: Experimental Design and Updating of Finite Element Models. Thesis No. 611, 1997.

U. Forssell: Properties and Usage of Closed-Loop Identification Methods. Thesis No. 641, 1997.

M. Larsson: On Modeling and Diagnosis of Discrete Event Dynamic systems. Thesis No. 648, 1997.

N. Bergman: Bayesian Inference in Terrain Navigation. Thesis No. 649, 1997.

V. Einarsson: On Verification of Switched Systems Using Abstractions. Thesis No. 705, 1998.

J. Blom, F. Gunnarsson: Power Control in Cellular Radio Systems. Thesis No. 706, 1998.

P. Spångéus: Hybrid Control using LP and LMI methods - Some Applications. Thesis No. 724, 1998.

M. Norrlöf: On Analysis and Implementation of Iterative Learning Control. Thesis No. 727, 1998.

A. Hagenblad: Aspects of the Identification of Wiener Models. Thesis No. 793, 1999.

F. Tjärnström: Quality Estimation of Approximate Models. Thesis No. 810, 2000.

C. Carlsson: Vehicle Size and Orientation Estimation Using Geometric Fitting. Thesis No. 840, 2000.

J. Löfberg: Linear Model Predictive Control: Stability and Robustness. Thesis No. 866, 2001.

O. Härkegård: Flight Control Design Using Backstepping. Thesis No. 875, 2001.

J. Elbornsson: Equalization of Distortion in A/D Converters. Thesis No. 883, 2001.

J. Roll: Robust Verification and Identification of Piecewise Affine Systems. Thesis No. 899, 2001.

I. Lind: Regressor Selection in System Identification using ANOVA. Thesis No. 921, 2001.

R. Karlsson: Simulation Based Methods for Target Tracking. Thesis No. 930, 2002.

P.-J. Nordlund: Sequential Monte Carlo Filters and Integrated Navigation. Thesis No. 945, 2002.

M. Östring: Identification, Diagnosis, and Control of a Flexible Robot Arm. Thesis No. 948, 2002.

C. Olsson: Active Engine Vibration Isolation using Feedback Control. Thesis No. 968, 2002.

J. Jansson: Tracking and Decision Making for Automotive Collision Avoidance. Thesis No. 965, 2002.

N. Persson: Event Based Sampling with Application to Spectral Estimation. Thesis No. 981, 2002.

D. Lindgren: Subspace Selection Techniques for Classification Problems. Thesis No. 995, 2002.

E. Geijer Lundin: Uplink Load in CDMA Cellular Systems. Thesis No. 1045, 2003.

M. Enqvist: Some Results on Linear Models of Nonlinear Systems. Thesis No. 1046, 2003.

T. Schön: On Computational Methods for Nonlinear Estimation. Thesis No. 1047, 2003.

F. Gunnarsson: On Modeling and Control of Network Queue Dynamics. Thesis No. 1048, 2003.

S. Björklund: A Survey and Comparison of Time-Delay Estimation Methods in Linear Systems. Thesis No. 1061, 2003. 
M. Gerdin: Parameter Estimation in Linear Descriptor Systems. Thesis No. 1085, 2004.

A. Eidehall: An Automotive Lane Guidance System. Thesis No. 1122, 2004.

E. Wernholt: On Multivariable and Nonlinear Identification of Industrial Robots. Thesis No. 1131, 2004.

J. Gillberg: Methods for Frequency Domain Estimation of Continuous-Time Models. Thesis No. 1133, 2004.

G. Hendeby: Fundamental Estimation and Detection Limits in Linear Non-Gaussian Systems. Thesis No. 1199, 2005.

D. Axehill: Applications of Integer Quadratic Programming in Control and Communication. Thesis No. 1218, 2005.

J. Sjöberg: Some Results On Optimal Control for Nonlinear Descriptor Systems. Thesis No. 1227, 2006.

D. Törnqvist: Statistical Fault Detection with Applications to IMU Disturbances. Thesis No. 1258, 2006.

H. Tidefelt: Structural algorithms and perturbations in differential-algebraic equations. Thesis No. 1318, 2007.

S. Moberg: On Modeling and Control of Flexible Manipulators. Thesis No. 1336, 2007.

J. Wallén: On Kinematic Modelling and Iterative Learning Control of Industrial Robots. Thesis No. 1343, 2008.

J. Harju Johansson: A Structure Utilizing Inexact Primal-Dual Interior-Point Method for Analysis of Linear Differential Inclusions. Thesis No. 1367, 2008.

J. D. Hol: Pose Estimation and Calibration Algorithms for Vision and Inertial Sensors. Thesis No. 1370, 2008.

H. Ohlsson: Regression on Manifolds with Implications for System Identification. Thesis No. 1382, 2008.

D. Ankelhed: On low order controller synthesis using rational constraints. Thesis No. 1398, 2009.

P. Skoglar: Planning Methods for Aerial Exploration and Ground Target Tracking. Thesis No. 1420, 2009.

C. Lundquist: Automotive Sensor Fusion for Situation Awareness. Thesis No. 1422, 2009.

C. Lyzell: Initialization Methods for System Identification. Thesis No. 1426, 2009.

R. Falkeborn: Structure exploitation in semidefinite programming for control. Thesis No. 1430, 2010.

D. Petersson: Nonlinear Optimization Approaches to $\mathcal{H}_{2}$-Norm Based LPV Modelling and Control. Thesis No. 1453, 2010.

Z. Sjanic: Navigation and SAR Auto-focusing in a Sensor Fusion Framework. Thesis No. 1464, 2011.

K. Granström: Loop detection and extended target tracking using laser data. Thesis No. 1465, 2011.

J. Callmer: Topics in Localization and Mapping. Thesis No. 1489, 2011.

F. Lindsten: Rao-Blackwellised particle methods for inference and identification. Thesis No. 1480, 2011.

M. Skoglund: Visual Inertial Navigation and Calibration. Thesis No. 1500, 2011.

S. Khoshfetrat Pakazad: Topics in Robustness Analysis. Thesis No. 1512, 2011.

P. Axelsson: On Sensor Fusion Applied to Industrial Manipulators. Thesis No. 1511, 2011.

A. Carvalho Bittencourt: On Modeling and Diagnosis of Friction and Wear in Industrial Robots. Thesis No. 1516, 2012.

P. Rosander: Averaging level control in the presence of frequent inlet flow upsets. Thesis No. 1527, 2012. 
N. Wahlström: Localization using Magnetometers and Light Sensors. Thesis No. 1581, 2013.

R. Larsson: System Identification of Flight Mechanical Characteristics. Thesis No. 1599, 2013.

Y. Jung: Estimation of Inverse Models Applied to Power Amplifier Predistortion. Thesis No. 1605, 2013.

M. Syldatk: On Calibration of Ground Sensor Networks. Thesis No. 1611, 2013.

M. Roth: Kalman Filters for Nonlinear Systems and Heavy-Tailed Noise. Thesis No. 1613, 2013.

D. Simon: Model Predictive Control in Flight Control Design - Stability and Reference Tracking. Thesis No. 1642, 2014.

J. Dahlin: Sequential Monte Carlo for inference in nonlinear state space models. Thesis No. 1652, 2014.

M. Kok: Probabilistic modeling for positioning applications using inertial sensors. Thesis No. 1656, 2014.

J. Linder: Graybox Modelling of Ships Using Indirect Input Measurements. Thesis No. 1681, 2014.

G. Mathai: Direction of Arrival Estimation of Wideband Acoustic Wavefields in a Passive Sensing Environment. Thesis No. 1721, 2015.

I. Nielsen: On Structure Exploiting Numerical Algorithms for Model Predictive Control. Thesis No. 1727, 2015.

C. Veibäck: Tracking of Animals Using Airborne Cameras. Thesis No. 1761, 2016.

N. Evestedt: Sampling Based Motion Planning for Heavy Duty Autonomous Vehicles. Thesis No. 1762, 2016.

H. Nyqvist: On Pose Estimation in Room-Scaled Environments. Thesis No. 1765, 2016.

Y. Zhao: Position Estimation in Uncertain Radio Environments and Trajectory Learning. Thesis No. 1772, 2017.

P. Kasebzadeh: Parameter Estimation for Mobile Positioning Applications. Thesis No. 1786, 2017.

K. Radnosrati: On Timing-Based Localization in Cellular Radio Networks. Thesis No. $1808,2018$.

G. Lindmark: Methods and Algorithms for Control Input Placement in Complex Networks. Thesis No. 1814, 2018.

M. Lindfors: Frequency Tracking for Speed Estimation. Thesis No. 1815, 2018.

D. Ho: Some results on closed-loop identification of quadcopters. Thesis No. 1826, 2018.

O. Ljungqvist: On motion planning and control for truck and trailer systems. Thesis No. 1832, 2019.

P. Boström-Rost: On Informative Path Planning for Tracking and Surveillance. Thesis No. 1838, 2019.

K. Bergman: On Motion Planning Using Numerical Optimal Control. Thesis No. 1843, 2019.

M. Klingspor: Low-rank optimization in system identification. Thesis No. 1855, 2019.

A. Bergström: Timing-Based Localization using Multipath Information. Thesis No. 1867, 2019.

F. Ljungberg: Estimation of Nonlinear Greybox Models for Marine Applications. Thesis No. 1880, 2020.

E. Hedberg: Control, Models and Industrial Manipulators. Thesis No. 1894, 2020. 


\section{FACULTY OF SCIENCE AND ENGINEERING}

Linköping Studies in Science and Technology, Licentiate Thesis No. 1897 Department of Electrical Engineering

Linköping University

SE-581 83 Linköping, Sweden

www.liu.se 